\title{
Fuel-In-Air
}

\section{FY07 Summary Report}
B. D. Hanson
W. Wu (BSC)
R. C. Daniel
P. J. MacFarlan
A. M. Casella
R. W. Shimskey
R. S. Wittman

September 2008

\section{Pacific Northwest}

NATIONAL LABORATORY 


\title{
DISCLAIMER
}

This report was prepared as an account of work sponsored by an agency of the United States Government. Neither the United States Government nor any agency thereof, nor Battelle Memorial Institute, nor any of their employees, makes any warranty, express or implied, or assumes any legal liability or responsibility for the accuracy, completeness, or usefulness of any information, apparatus, product, or process disclosed, or represents that its use would not infringe privately owned rights. Reference herein to any specific commercial product, process, or service by trade name, trademark, manufacturer, or otherwise does not necessarily constitute or imply its endorsement, recommendation, or favoring by the United States Government or any agency thereof, or Battelle Memorial Institute. The views and opinions of authors expressed herein do not necessarily state or reflect those of the United States Government or any agency thereof.

\author{
PACIFIC NORTHWEST NATIONAL LABORATORY \\ operated by \\ BATTELLE \\ for the \\ UNITED STATES DEPARTMENT OF ENERGY \\ under Contract DE-AC05-76RL01830
}

Printed in the United States of America
Available to DOE and DOE contractors from the Office of Scientific and Technical Information,
P.O. Box 62, Oak Ridge, TN 37831-0062;
ph: (865) 576-8401
fax: (865) 5765728
email: reports@adonis.osti.gov

\begin{abstract}
Available to the public from the National Technical Information Service, U.S. Department of Commerce, 5285 Port Royal Rd., Springfield, VA 22161 ph: (800) 553-6847 fax: (703) 605-6900

email: orders@nits.fedworld.gov

online ordering: http://www.ntis.gov/ordering.htm
\end{abstract}

This document was printed on recycled paper. 


\section{Fuel-In-Air FY07 Summary Report}
B. D. Hanson
W. Wu (BSC)
R. C. Daniel
P. J. MacFarlan
A. M. Casella
R. W. Shimskey
R. S. Wittman

September 2008

Prepared for

the U.S. Department of Energy

under Contract DE-AC05-76RL01830

Pacific Northwest National Laboratory

Richland, Washington 99352 


\section{Summary}

This report presents the results of testing conducted by Pacific Northwest National Laboratory (PNNL) in support of Bechtel SAIC Co., LLC (BSC) during fiscal year 2007. The objectives of the testing were to provide data to determine if high burnup (HBU) Commercial Spent Nuclear Fuel (CSNF) (i.e., burnup in the range 45 to $80 \mathrm{GWd} / \mathrm{MTU}$ ) will behave differently than lower burnup fuels tested previously, to determine the fractional release of fuel from a spent fuel rod upon cladding failure (i.e., burst scenario), and to determine the fraction and particle-size distribution (PSD) of oxidized fuel that may become airborne under off-normal scenarios in the surface facilities at the proposed repository at Yucca Mountain.

The Fuel-In-Air Test Apparatus (FIATA) was designed primarily to determine the PSD of fuel in a well-fluidized air stream. The use of cascade impactors or optical particle counters (OPCs) to determine these distributions required the inclusion of balance air and may have affected the detection of particles by these instruments. The results of a literature review and the testing follows.

- A literature review focusing on the formation of the High Burnup Structure (HBS) and properties of fuel finds that, overall, no impact of HBS formation on fuel distribution is expected (Section 1.1).

- $\quad \mathrm{HBU}$ fuel known to contain $\mathrm{HBS}$ appears to have oxidized completely to $\mathrm{U}_{3} \mathrm{O}_{8}$ within 30 days for both tests operating at $450^{\circ} \mathrm{C}$ and $500^{\circ} \mathrm{C}$. However, similar samples oxidized at $350^{\circ} \mathrm{C}$ and $400^{\circ} \mathrm{C}$ appear to have oxidized only to the plateau, and no powder formation was observed (Section 2.3). There is an apparent contradiction with literature data on fuel oxidized at $397^{\circ} \mathrm{C}$ that converted to $\mathrm{U}_{3} \mathrm{O}_{8}$ in hours. Additional tests are planned for FY08 to resolve this apparent contradiction.

- The FIATA was used with rod segments of fuel to determine the quantity of fuel that may be released if a rod were to burst, possibly as a result of a drop scenario.

o A PWR fuel with a burnup of $45 \mathrm{GWd} / \mathrm{MTU}$ was tested three separate times using different orientations. The fractional release calculated using the OPC and a calculated global detection efficiency of 0.065 (Section 6.3.8) ranges from $1 \times 10^{-5}$ to $8 \times 10^{-5}$ (Section 4.2.1).

o Three different segments of HBU fuel from a BWR were tested multiple times. In some instances, the maximum flow was limited below 500 SCCM and a maximum pressure of $130 \mathrm{psig}$ because the fuel/clad gap was closed, and the cracks in the fuel pellets were very tight. Release fractions from these fuels ranged from $9 \times 10^{-7}$ to $8 \times 10^{-6}$ (Sections 4.2.2 through 4.2.4). In the one instance where high flow was achieved, the release fraction was $2 \times 10^{-5}$.

o In one instance, a cascade impactor was used in conjunction with gamma energy analysis to determine the fractional release. The test with the cascade impactor was performed first and yielded a fractional release one-fourth that observed using the OPC. This seems to indicate that the global efficiency of 0.065 is valid since a factor of four difference is within the range of repeat tests (Section 4.2.2).

o Based on the releases observed, there is no significant difference in either the release fraction or the PSD between HBU fuels containing HBS and lower burnup fuels (Section 4.3, Appendix C). 
- The FIATA was used with powders of oxidized spent fuels to determine fractional release and PSD. Based on mass-difference measurements, the total release fractions range from 0.09 to 0.75 for samples subjected to flow rates up to $1000 \mathrm{SCCM}$ (greater than $2.1 \mathrm{~m} / \mathrm{s}$ linear flow). However, the expected flow rates within a failed container are expected to be closer to $0.1 \mathrm{~m} / \mathrm{s}$ (BSC 2007a). For conditions more applicable to these scenarios, the release fractions obtained using OPCs and a global detection efficiency of 0.065 range from 0.01 to 0.65 . In all cases, virtually no release was detected in this lower flow range except under the transient condition when the flow control was switched from one controller to another (Section 5.2 and Section 5.3).

- While differences in PSD were observed, the differences were not large and appeared to be a function of the post-irradiation grain size and the oxidation temperature (Section 5.4).

- Justification for the global OPC efficiency of 0.065 is presented in Section 6. While this efficiency appears to underestimate powder releases by up to two orders of magnitude, this is most likely because of differences (density, morphology, sticking potential, etc.) between the silica powder and the CSNF oxidized powders. The denser CSNF powders would be expected to settle more rapidly in a flowing air stream. Even though fuel was released from the holder, the experiments suggest that the overwhelming majority of fuel will settle out or stick to other materials quickly, thus limiting the fraction that remains airborne. A modification to the FIATA is proposed for additional testing to be performed in FY08 to eliminate the issues that prevent release fractions from being determined with certainty with the current system (Section 6.3.8).

- Calculations were performed in Section 7 to determine if the increased linear velocity as air exits the frits used to hold the fuel imparted enough momentum for particles to be ejected from the holder at lower superficial velocities. Using conservative assumptions and analyses, it is shown that the high release of CSNF oxidized powders was not a result of higher flow rates through the frits. The modified FIATA for FY08 will not need frits to hold the fuel to avoid this complication. 


\section{Quality Assurance}

The Pacific Northwest National Laboratory (PNNL) project that performed these studies was required by the U.S. Department of Energy (DOE) Office of Repository Development and Bechtel SAIC Company, LLC (BSC), to develop and implement a Quality Assurance (QA) Program that addressed the requirements of DOE/RW-0333P, Office of Civilian Radioactive Waste Management (OCRWM) Quality Assurance Requirements and Description (QARD), as applicable to the PNNL scope of work. In response to this requirement, the Nuclear Quality Assurance Requirements and Description (NQARD) QA Program was imposed upon the experimental studies described in this document.

The PNNL NQARD QA Program provided an effective management system for conducting and verifying quality-affecting activities in a planned, controlled, and traceable manner. The adequacy, effectiveness, and implementation of the NQARD QA Program have been verified by multiple DOE clients. The NQARD QA Program was audited and approved by the OCRWM Office of Quality Assurance in August 1998 as it related to PNNL work that supports OCRWM's Yucca Mountain Project. The OCRWM Office of Quality Assurance lists PNNL and the NQARD QA Program on their Qualified Supplier Listing. As a qualified supplier, OCRWM and BSC attest that the PNNL NQARD QA Program is adequate and is being effectively implemented. The PNNL NQARD QA Program has also been audited by BSC on a triennial basis in 2001, 2004, and 2006 to confirm that the PNNL QA Program has continued its effective implementation.

Based on the acceptance of the PNNL NQARD QA Program by the OCRWM Office of Quality Assurance in August 1998 and the subsequent evaluations and approval of this QA program by OCRWM and BSC in 2001, 2004, and 2006, the data in this technical report are considered qualified and acceptable for use with the following exceptions.

The only exceptions preventing all data in this report from being fully qualified are the reported mass of fuel (both powders and rod segments) and the estimates of the fuel mass in the cascade impactors obtained by gamma energy analysis. The balances used in the hot cells were calibrated by the same vendor (one that is on the OCRWM Qualified Supplier Listing) as the other balances (such as those used for the silica calibration tests), but the hot-cell balances were not part of the Purchase Order that was reviewed by the Project Quality Engineer as required. The procedure and standards used for the gamma energy analysis are part of the Analytic Service Organization (ASO), a supplier to the PNNL Project. However, the audit of ASO had not been performed to keep them on the PNNL Evaluated Supplier Listing, so these results are considered for information only.

All QA records generated as a result of implementing the NQARD QA Program for the studies described in this technical report are maintained as permanent records within the PNNL and DOE records management systems. 



\section{Glossary of Definitions and Acronyms}

\begin{tabular}{|c|c|}
\hline AED & Aerodynamic Equivalent Diameter \\
\hline ARF & Airborne Release Fraction \\
\hline ATM & Approved Testing Material \\
\hline BSC & Bechtel SAIC Co., LLC \\
\hline BWR & boiling water reactor \\
\hline CRWMS & Civilian Radioactive Waste Management Service \\
\hline CSNF & Commercial Spent Nuclear Fuel \\
\hline DOE & U.S. Department of Energy \\
\hline FGR & Fission-gas release \\
\hline FIATA & Fuel-In-Air Test Apparatus \\
\hline HBS & high burnup structure \\
\hline $\mathrm{HBU}$ & high burnup \\
\hline HEPA & high-efficiency particulate air \\
\hline $\mathrm{M} \& \mathrm{O}$ & Management and Operations \\
\hline NIST & National Institute of Science and Technology \\
\hline $\mathrm{O} / \mathrm{M}$ & oxygen-to-metal (ratio) \\
\hline $\mathrm{OPC}$ & optical particle counter \\
\hline PID & proportional-integral-differential \\
\hline PNNL & Pacific Northwest National Laboratory \\
\hline PSD & particle-size distribution \\
\hline PSL & polystyrene latex \\
\hline PWR & pressurized water reactor \\
\hline
\end{tabular}




$\begin{array}{ll}\text { RF } & \text { Respirable Fraction } \\ \text { RPL } & \text { Radiochemical Processing Laboratory } \\ \text { SAL } & \text { Shielded Analytical Laboratory } \\ \text { SEM } & \text { scanning electron microscopy } \\ \text { SFA } & \text { spent-fuel assembly } \\ \text { TAD } & \text { transportation, ageing and disposal } \\ \text { TGA } & \text { thermal gravimetric analysis } \\ \text { YMP } & \text { Yucca Mountain Project }\end{array}$

\section{Unit Abbreviations}

$\begin{array}{ll}{ }^{\circ} \mathrm{C} & \text { degrees Centigrade } \\ \mathrm{g} / \mathrm{cm}^{3} & \text { grams per cubic centimeter (density) } \\ \mathrm{GWd} & \text { Gigawatt day } \\ \mathrm{mL} / \mathrm{g} & \text { milliliters per gram } \\ \mathrm{MTU} & \text { metric ton of uranium } \\ \mathrm{SCCM} & \text { standard cubic centimeters per minute } \\ \mathrm{SLM} & \text { standard liters per minute }\end{array}$




\section{Contents}

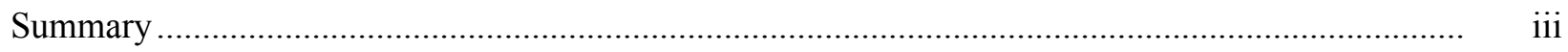

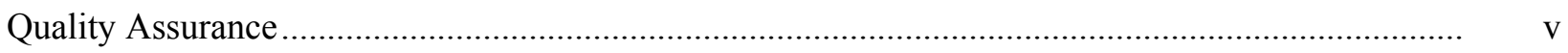

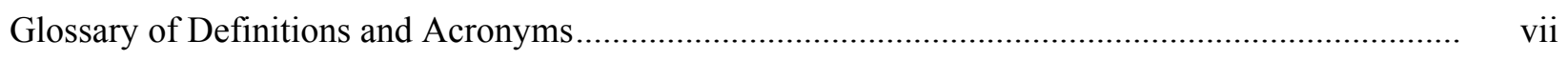

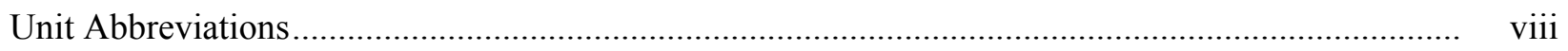

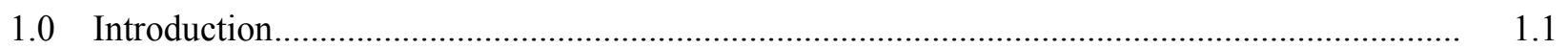

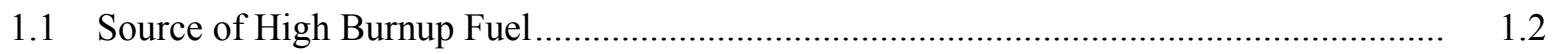

1.1.1 High Burnup Fuel Characteristics .................................................................. 1.2

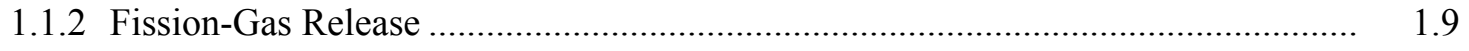

1.1.3 Fission Volatile Radionuclide Release ............................................................. 1.12

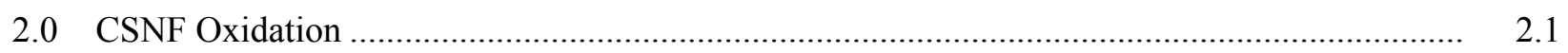

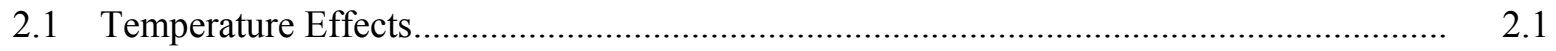

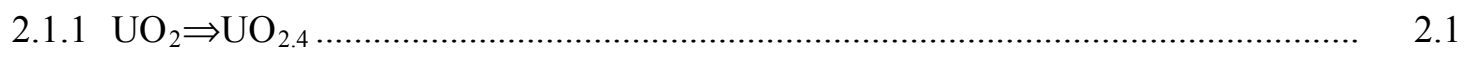

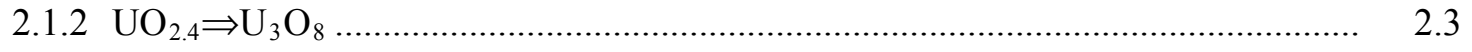

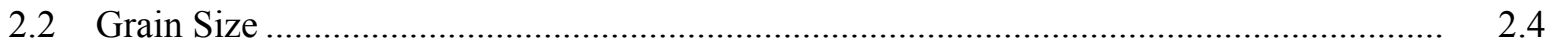

2.2.1 Initial Grain Size and Growth ............................................................................ 2.5

2.2.2 Post-Oxidation Particle Size.............................................................................. 2.6

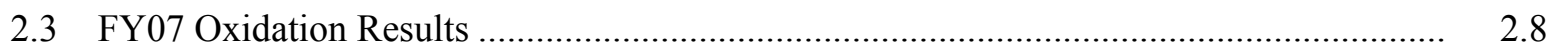

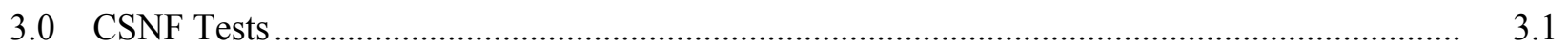

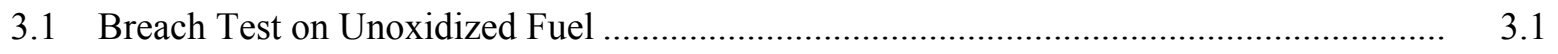

3.2 Suspension Test on Oxidized Fuel Powders ............................................................ 3.2

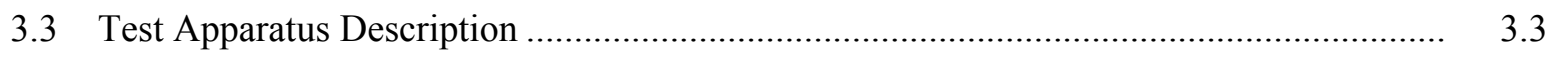

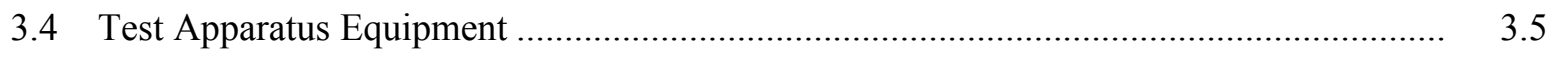

3.5 Preparation of the Particle Collection/Analysis System ............................................... 3.9

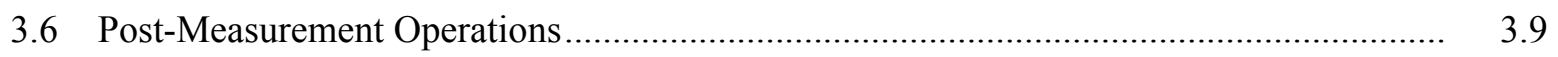

3.7 Estimate of Release in Optical-Particle-Counter Tests................................................. 3.10

3.8 Estimates of Mass Release and Release Fraction ......................................................... 3.14

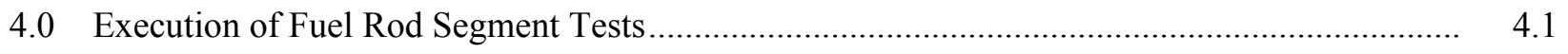

4.1 Overall Results of CSNF Rod Segment Tests ........................................................... 4.3

4.2 Individual Results of CSNF Rod Segment Tests......................................................... 4.6

4.2.1 ATM-106 Rod Segment Test Results …............................................................. 4.7

4.2.2 HBU 539B3 Rod Segment Test Results....................................................... 4.8

4.2.3 HBU 539D5 Rod Segment Test Results ...................................................... 4.10

4.2.4 HBU 539F5 Rod Segment Test Results ........................................................... 4.11 
4.3 Rod-Segment Release-Fraction Results.............................................................. 4.12

5.0 Execution of Oxidized Fuel Powder Tests......................................................................... 5.1

5.1 Overall Results of CSNF Oxidized Powder Tests .......................................................... 5.2

5.2 Individual Results of CSNF Oxidized Powder Tests....................................................... 5.3

5.2.1 109-A450 Powder Test Results ......................................................................... 5.4

5.2.2 109-A500 Powder Test Results ........................................................................ 5.5

5.2.3 105-LOW Powder Test Results......................................................................... 5.6

5.2.4 105-HIGH Powder Test Results............................................................................. 5.6

5.3 Oxidized Powder Release Fraction Results Discussion............................................... 5.7

5.4 Particle Size of Oxidized Powders............................................................................ 5.9

6.0 Calibration of Total Versus Detected Release Using Silica Powders.................................... 6.1

6.1 Execution of Silica Calibration Tests .......................................................................... 6.2

6.2 Analysis of Raw Silica Calibration Data ..................................................................... 6.5

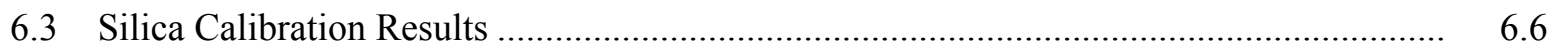

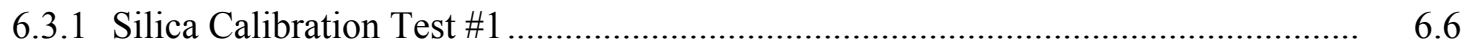

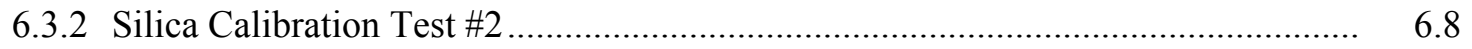

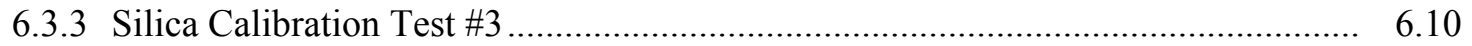

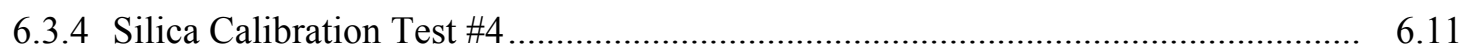

6.3.5 Examination of Release Particle Size as a Function of Gas Flow Rate .................. 6.13

6.3.6 Summary of Silica Calibration Test Results …................................................ 6.14

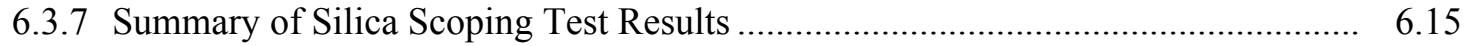

6.3.8 Estimate of Global Counting Efficiency ......................................................... 6.16

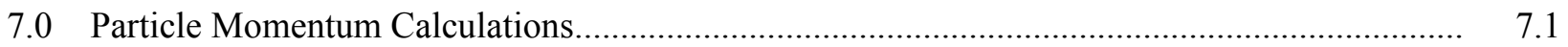

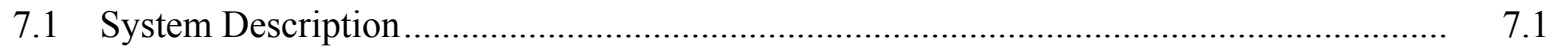

7.2 Force Balances and Trajectory Calculations ..................................................................

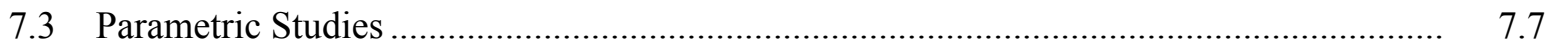

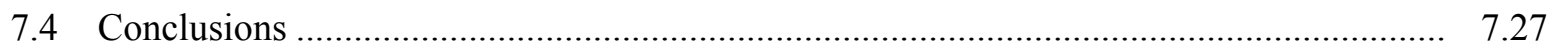

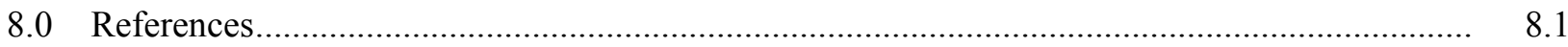

Appendix A: Mass Release from CSNF Rod Tests …................................................................. A.1

Appendix B: Mass Release from CSNF Powder Tests .......................................................... B.

Appendix C: Particle-Size Distributions from CSNF Rod Tests ................................................ C.1

Appendix D: Particle-Size Distributions for CSNF Powder Tests ............................................ D. 


\section{Figures}

1.1. Radial Burnup Profile in CSNF with Pellet-Average Burnup of 44.3 GWd/MTU .................. 1.3

1.2. Rim Thickness as a Function of Burnup (GWd/MTU) .................................................... 1.4

1.3. Grain-Size Distributions of Recrystallized Fuel with a Pellet-Average Burnup of 98 GWd/

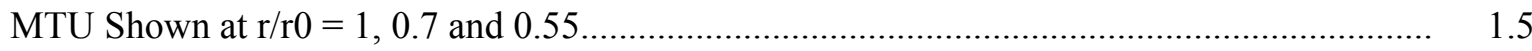

1.4. Fractional Porosity as a Function of Radial Position......................................................... 1.6

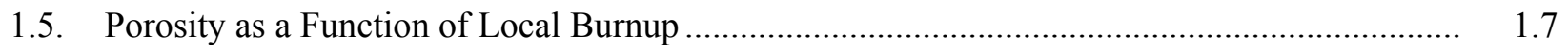

1.6. Relative Hardness as a Function of Radial Position ........................................................ 1.7

1.7. Relative Hardness as a Function of Fractional Porosity ................................................... 1.8

1.8. Fracture Toughness as a Function of Radial Position .................................................... 1.9

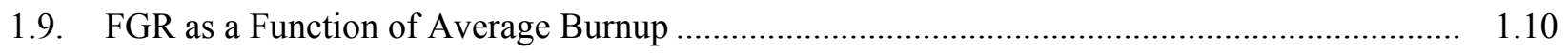

1.10. FGR from PWR Fuel as a Function of Burnup, Solid Line—Best Fit; Dashed Line-

Bounding or Pessimistic Estimate ................................................................................. 1.10

1.11. Cumulative Fractional Release of $\mathrm{Kr}$ at the Ramp and Isothermal Annealing Temperature of $1800^{\circ} \mathrm{C}$

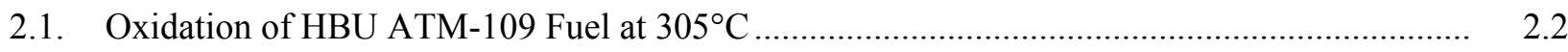

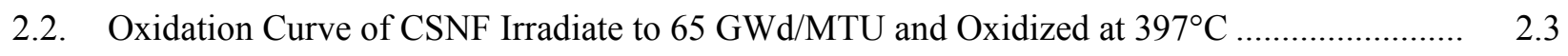

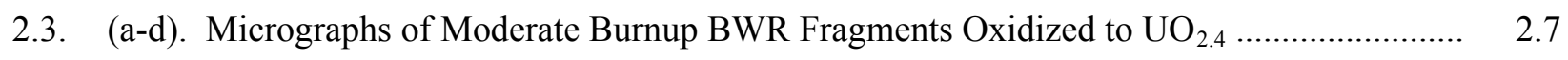

2.4. (a-c). Micrographs of Moderate Burnup BWR Fragments Oxidized to $\mathrm{U}_{3} \mathrm{O}_{8}$ at a) $283^{\circ} \mathrm{C}$,

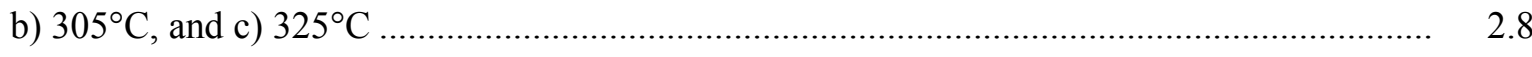

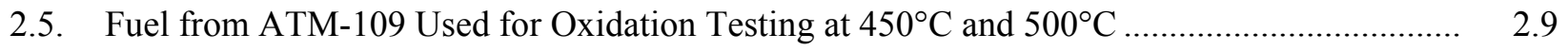

2.6. Schematic of Oxidation Apparatus .......................................................................... 2.11

2.7. Weighing of Sample After Oxidation....................................................................... 2.11

2.8. ATM-109 Fuel Fragment Oxidized for Approximately 30 Days at $500^{\circ} \mathrm{C}$ Disintegrates into Powder

2.9. SEM Images of ATM-109 Fuel Oxidized at $450^{\circ} \mathrm{C}$ for Approximately 30 Days ................... 2.14

2.10. SEM Images of ATM-109 Fuel Oxidized at $500^{\circ} \mathrm{C}$ for Approximately 30 Days ................... 2.15

3.1. Example of Pellet Cracking and Flow Path for ATM-106 rod NBD-107 ............................. 3.2 
3.2. The Sample Holder (A) Consists of a Gas Line Inlet Connected to a Compressed Dry Air Gas Cylinder and a Swagelok ${ }^{\circledR}$ Fitting to Secure the Fuel Segment or Sample Boat Being Tested

3.3. Fuel-in-Air Test Apparatus Setup Used for Testing CSNF Rod Segments and Powders at the RPL's SAL

4.1. Photographs of each End of the Four CSNF Rod Segments Tested: a-b) ATM-106, c-d) HBU 539B3, e-f) HBU 539D5, g-h) HBU539F5

6.1. Schematic of Modified FIATA Employed for Silica Calibration Test \#4.....

6.2. Silica Calibration Correlation Curve Showing the Mass Detected at the OPC as a Function of the Mass Lost from the Sample Boat for Silica Calibration Test \#1.....

6.3. Silica Calibration Correlation Curve Showing the Mass Detected at the OPC as a Function of the Mass Lost from the Sample Boat Silica Calibration Test \#1

6.4. Silica Calibration Correlation Curve Showing the Mass Detected at the OPC as a Function of the Mass Lost from the Sample Boat Silica Calibration Test \#3

6.5. Silica Calibration Correlation Curve Showing the Mass Detected at the OPC as a Function of the Mass Lost from the Sample Boat Silica Calibration Test \#4

6.6. Particle Size as a Function of Flow Rate for (A) Silica Calibration Test \#1, Using the Typical FIATA Setup over Flow Rates Between 100 to 1000 SCCM and (B) Silica Calibration Test \#4, Using a Direct Connection Between Sample Boat and OPC over 100 to 1000 SCCM

7.1. Flow System Being Modeled in this Section

7.2. Post-Frit (Zone 1) Force Balance on a Particle

7.3. Force Balance on a Particle Engulfed by an Upward Air Stream 


\section{Tables}

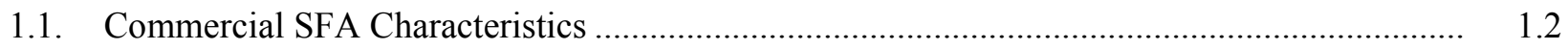

1.2. Comparison of Rim-Zone Microstructural Properties and Typical Properties of Low Burnup $\mathrm{UO}_{2}$ Fuel

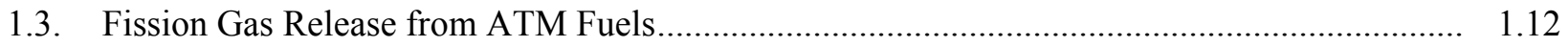

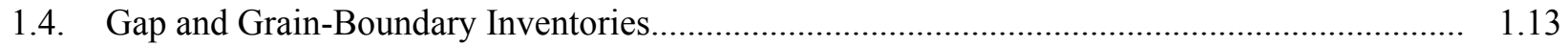

3.1. Summary of Major Equipment Used for SAL FIATA .....................................................

3.2. Operational Parameters for Met-One Instruments, Inc., Model 61636 Sixteen-Channel

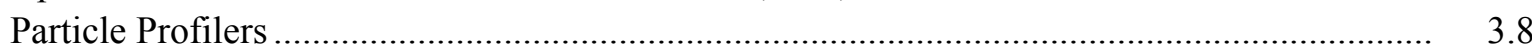

3.3. Operational Parameters for Sioutas Cascade Impactors ................................................ 3.8

3.4. Particle-Size Settings for the 16-Channel OPC ................................................................. 3.10

3.5. Average Particle Size for each OPC Channel ............................................................... 3.11

4.1. Estimated Dimensions and Mass of Fuel Rod Segments Used in SAL FIATA Testing ............

4.2. Summary of CSNF Rod Segment Testing Parameters ............................................................

4.3. Summary of CNSF Rod Segment Test Release Results...................................................

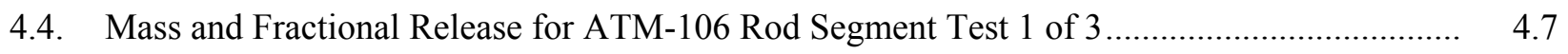

4.5. Mass and Fractional Release for ATM-106 Rod Segment Test 2 of 3 ..................................

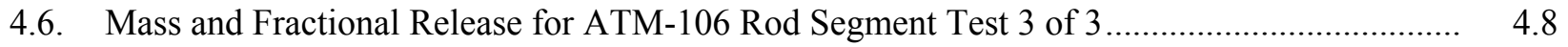

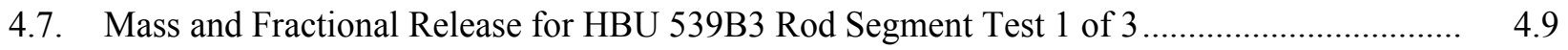

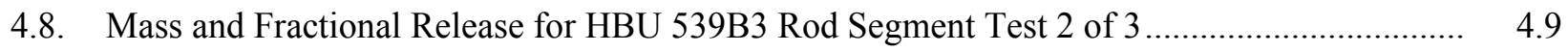

4.9. Mass and Fractional Release for HBU 539B3 Rod Segment Test 3 of 3 ................................. 4.9

4.10. Mass and Fractional Release for the HBU 539D5 Rod Segment Test ................................. 4.10

4.11. Mass and Fractional Release for HBU 539F5 Rod Segment Test 1 of $2 \ldots \ldots \ldots \ldots \ldots \ldots \ldots \ldots \ldots \ldots \ldots \ldots . . . . . . . . .11$

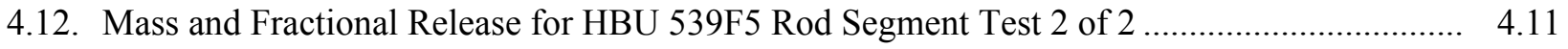

4.13. Estimated Gas-Flow Velocity Inside Porous Interior of Fuel Rod Segments ......................... 4.13

5.1. Mass of Oxidized Fuel Used in Powder Tests ........................................................................ 
5.2. Summary of CSNF Oxidized Powder Testing Parameters.................................................. 5.2

5.3. Summary of CNSF Oxidized Powder Test Release Results ............................................. 5.2

5.4. Mass and Fractional Release for the CSNF Powder Test...................................................

5.5. Mass and Fractional Release for the 109-A450 Powder Test...............................................

5.6. Mass and Fractional Release for the 109-A500 Powder Test............................................... 5.5

5.7. Mass and Fractional Release for the 105-LOW Powder Test ................................................ 5.6

5.8. Mass and Fractional Release for the 105-HIGH Powder Test .......................................... 5.7

5.9. Estimated Linear Gas Flow Velocity Inside Powder Sample Boat ....................................... 5.8

5.10. Particle-Size Distribution for the Sum of All Non-Background Release Events Observed in Oxidized Powder Tests ............................................................................................ $\quad 5.10$

6.1. Properties of the Particle Size Standard Used in Silica Calibration Testing .......................... 6.2

6.2. Event-to-Event Results of Silica Calibration for Test \#1, Which Examined Counting Efficiencies over Flow Rates Between 100 and 1000 SCCM Without any Mechanical Action

6.3. Event-to-Event Results of Silica Calibration for Test \#2, Which Examined Counting Efficiencies over Flow Rates Between 100 and 1000 SCCM with Mechanical Action.

6.4. Event-to-Event Results of Silica Calibration for Test \#3, Which Examined Counting Efficiencies Using Flow Set Points and Mechanical Actions Similar to Those Used in Hot Cell Testing

6.5. Event-to-Event Results of Silica Calibration for Test \#4, Which Employed a Direct Connection Between Sample Boat and OPC

6.6. Summary of Silica Calibration Test Results........................................................................ 6.14

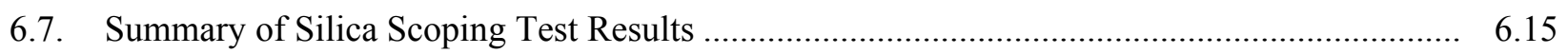

6.8. Summary of Silica Scoping Test Results ....................................................................

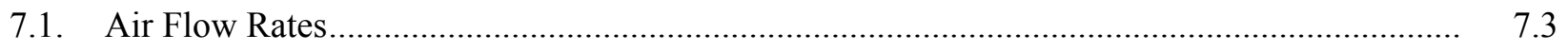

7.2. Maximum Particle Size with an Initial Upward Acceleration ............................................. 7.8

7.3. Maximum Particle Size for which the Final Particle Velocity is Greater than 0 for each Volumetric Flow Rate

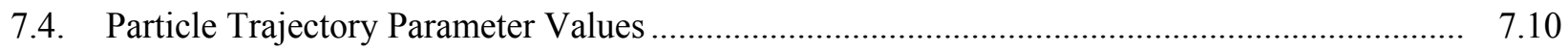

7.5. Maximum Vertical Height Achieved by Particles in a 100-SCCM Flow ................................ 7.11 
7.6. Adjustment of $h_{s}$ Value for a 100-SCCM Flowrate .........................................................

7.7. Adjustment of $h_{s}$ Value for a 200-SCCM Flowrate …....................................................

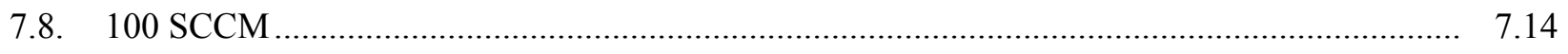

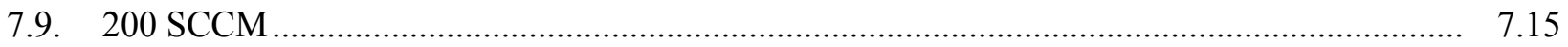

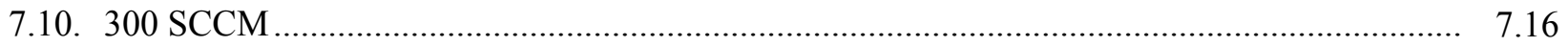

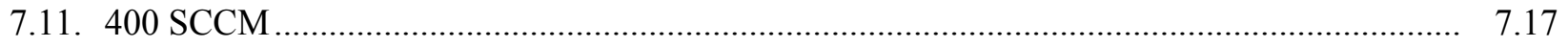

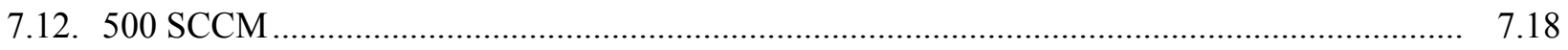

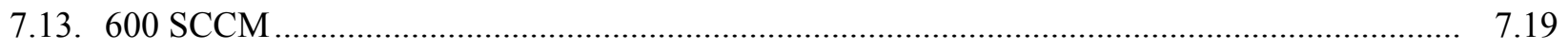

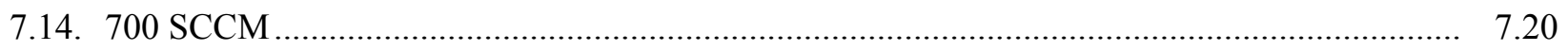

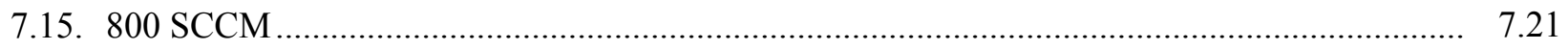

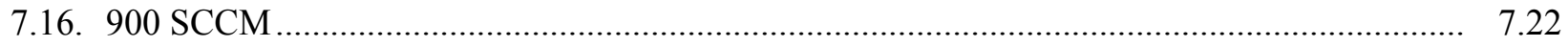

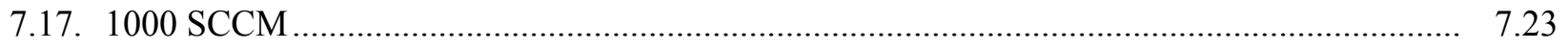

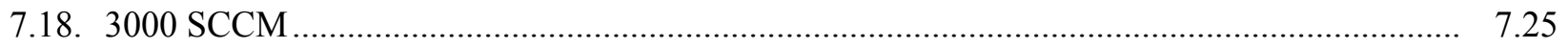




\subsection{Introduction}

When commercial spent nuclear fuel (CSNF) is handled in a dry environment, whether as fuel assemblies, canned, or within a container, one possible mechanism for radionuclide release is from oxidation of the CSNF exposed to air. The fuel in intact rods with no through-wall cladding failures or in rods in sealed cans or containers that maintain an inert environment would be prevented from oxidizing. However, in a drop accident scenario, it is possible that the cladding could fracture, and cans or containers could breach, allowing oxygen to contact the fuel (Sprung et al. 2000). Upon clad breach, it is expected that the rod would rapidly depressurize, releasing its fill gas (e.g., $\mathrm{He}$ ) and fission gases (e.g., $\mathrm{Kr}, \mathrm{Xe}$ ) that have been released from the fuel matrix, depending on the size of the cladding defect and fuel burnup characteristics (Einziger and Beyer 2007). It has been conservatively estimated (Anderson et al. 1996) that $30 \%$ of the fission gas inventory of a failed rod is released as the result of a cladding breach. It is also possible for fuel fines to be ejected as the high-pressure fill and fission gases rapidly escape through the defect. As the fuel oxides and forms $\mathrm{U}_{3} \mathrm{O}_{8}$, it swells and causes further clad unzipping, potentially releasing the fine-powdered $\mathrm{U}_{3} \mathrm{O}_{8}$ and allowing additional fission gas release.

To determine potential dose consequences from radionuclide releases, it is necessary to determine the fraction of fuel or fuel fines that may become airborne or the airborne release fraction (ARF) as well as the fraction that is respirable $(\mathrm{RF})$. The RF is defined as the fraction of airborne radionuclides as particles that can be transported through air and inhaled into the human respiratory system and is commonly assumed to include particles of 10- $\mu \mathrm{m}$ aerodynamic equivalent diameter (AED) or less (DOE 1994). The AED refers to the diameter of a sphere of density $1 \mathrm{~g} \mathrm{~cm}^{-3}$ that exhibits the same terminal velocity of the particle in question. For releases from oxidation of the spent fuel, Davis et al. (1998) recommend using an ARF of $12 \%$, with an RF of $1 \%$ yielding a total fraction of fuel available for respiration of $0.12 \%$ or 0.0012. The ARF is derived from experimental work by Iwasaki et al. (1968) on unirradiated $\mathrm{UO}_{2}$ pellets, and the RF is based on work compiled in the U.S. Department of Energy (DOE) Handbook (DOE 1994). However, the analysis by Davis et al. (1998) was for an aircraft accident where oxidized fuel could be picked up by the wind and dispersed in the open environment and is, therefore, considered overly conservative for the conditions expected in the surface facilities at the proposed Yucca Mountain repository. In these facilities, it is likely that any failed fuel assemblies would be in a relatively confined environment and would have a very low chance of being disturbed. Based on initial calculations and a review of ARFs reported in literature for a variety of oxidized powders (BSC 2007a), the ARF for oxidized CSNF is anticipated to be approximately $2 \times 10^{-3}$.

A number of factors contribute to these estimates and will be examined as part of a combined modeling and testing effort with Bechtel SAIC Co. LLC (BSC) and Pacific Northwest National Laboratory (PNNL). The most fundamental factors determining airborne release are the particle size, density, morphology, and the velocity of the airflow or forces for resuspension. The particle size, in turn, will be dictated by the post-irradiation grain size of the CSNF and whether the fuel has oxidized to $\mathrm{U}_{3} \mathrm{O}_{8}$. The oxidation of $\mathrm{UO}_{2}$ to $\mathrm{U}_{3} \mathrm{O}_{8}$ is a function of the time at temperature, initial grain size, burnup, and oxygen availability. This document addresses the current state of knowledge for each of these factors to serve as a guide for the modeling and testing effort. 


\subsection{Source of High Burnup Fuel}

Spent nuclear fuel exceeding 45 gigawatt days per metric ton of uranium (GWd/MTU) is classified as high burnup (HBU) fuel (Jain et al. 2004, p.1-1). The assembly-average burnup level for CSNF was less than $40 \mathrm{GWd} / \mathrm{MTU}$ before the early 1990s, with the exception of lead test assemblies. Today, the majority of the spent fuel discharged has a burnup level in excess of $45 \mathrm{GWd} / \mathrm{MTU}$ (McKinnon and Cunningham 2003, Section 2.2). The current reactor operating burnup limit is $62 \mathrm{GWd} / \mathrm{MTU}$ (peak rod), and the nuclear industry seeks to increase peak rod burnup from 62 to $75 \mathrm{GWd} / \mathrm{MTU}$. It is estimated that 30 percent of the spent nuclear fuel could be classified as HBU fuel for the potential Yucca Mountain repository (Jain et al. 2004, p.1-1).

Spent-fuel assemblies (SFAs) from pressurized water reactors (PWRs) and boiling water reactors (BWRs) are considered with the four different combinations of initial enrichment, burnup, and decay time for the source term in the preclosure consequence analysis (BSC 2007b), as shown in Table 1.1. Since industry is working towards increasing the peak rod burnup, the analyses for the preclosure safety analysis consider the effects of events involving a PWR assembly with a peak burnup of $80 \mathrm{GWd} / \mathrm{MTU}$ and a BWR assembly with a peak burnup of $75 \mathrm{GWd} / \mathrm{MTU}$. Because no CSNF currently exists with those properties, this report is used to estimate the behavior of these higher burnup fuels with respect to oxidation and potential release from breached rods.

Table 1.1. Commercial SFA Characteristics

\begin{tabular}{|l|c|c|c|c||}
\hline \multicolumn{1}{|c|}{ SFA } & $\begin{array}{c}\text { Initial } \\
\text { Enrichment } \\
\text { (Percent) }\end{array}$ & $\begin{array}{c}\text { Initial } \\
\text { MTHM/assembly }\end{array}$ & $\begin{array}{c}\text { Burnup } \\
\text { (GWd/MT } \\
\text { U) }\end{array}$ & $\begin{array}{c}\text { Decay Time } \\
\text { (Years) }\end{array}$ \\
\hline Representative PWR ${ }^{\mathrm{a}}$ & 4.2 & 0.475 & 50 & 10 \\
\hline Maximum PWR & 5.0 & 0.475 & 80 & 5 \\
\hline Representative BWR & 4.0 & 0.200 & 50 & 10 \\
\hline Maximum BWR & 5.0 & 0.200 & 75 & 5 \\
\hline Sources: ${ }^{\mathrm{a}}{ }^{\mathrm{b}}$ BSC 2007b, Table 12 & & \\
\hline
\end{tabular}

\subsubsection{High Burnup Fuel Characteristics}

When the cross-section averaged burnup of a fuel rod exceeds about $40 \mathrm{GWd} / \mathrm{MTU}$, the surface microstructure of the $\mathrm{UO}_{2}$ fuel pellet changes, which is known as the rim or high burnup structure (HBS). The local burnup is dependent on the radial location within a fuel pellet, with the higher burnup at the surface of the pellet, as shown in Figure 1.1. The higher burnup and cooler temperatures near the pellet surface result in the formation of the HBS. The thickness of the rim increases with the burnup dramatically. There are several characteristics in the rim zone: smaller grain size ( 0.1 to $0.3 \mu \mathrm{m})$, higher porosity (up to more than 20\%), and the larger pore size (a few microns). These changes become more significant as burnup increases. Une et al. (2000, p.62) reported that the pellet size and fission rate hardly influence the HBS formation. Several characteristics of HBU fuel are discussed below. 


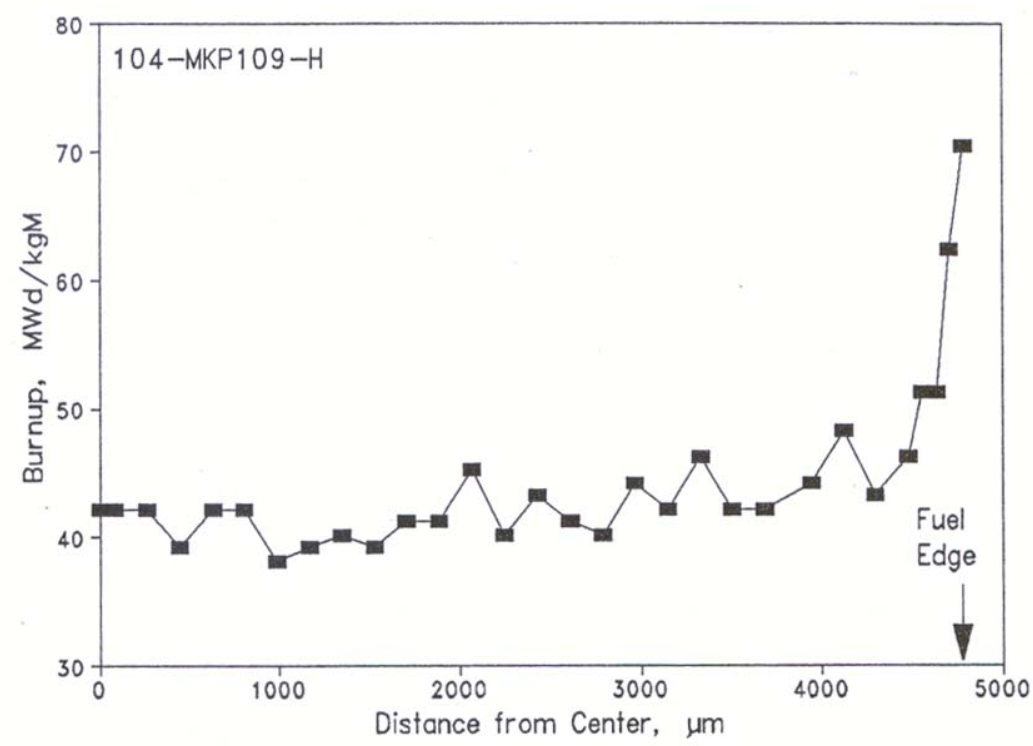

Figure 1.1. Radial Burnup Profile in CSNF with Pellet-Average Burnup of $44.3 \mathrm{GWd} / \mathrm{MTU}$ (Guenther et al. 1991a, Figure 7.15)

Rim Properties-The properties of a $\mathrm{UO}_{2}$ fuel pellet are compared between the rim zone of HBU fuel and low burnup fuel in Table 1.2 (Jernkvist and Massih 2004, p.11).

Table 1.2. Comparison of Rim-Zone Microstructural Properties and Typical Properties of Low Burnup $\mathrm{UO}_{2}$ Fuel

\begin{tabular}{|c|c|c|}
\hline Fuel Material Property & Rim Zone & Low Burnup Fuel \\
\hline Density $\left(\mathrm{kg} / \mathrm{m}^{3}\right)$ & 9670 & 10250 \\
\hline Porosity (volume fraction) & 0.10 & 0.04 \\
\hline Grain size $(\mu \mathrm{m})$ & 0.3 & 10 \\
\hline Intergranular bubble size $(\mathrm{nm})$ & 2.0 & 20 \\
\hline
\end{tabular}

Rim Thickness-Johnson et al. (2005) recently provided a summary of the rim thickness as a function of average burnup based on the literature review, as shown in Figure 1.2. There are three equations in the figure representing the different aspects of the rim-thickness calculation. The best fit shown in Figure 1.2 is represented by (Koo et al. 2001, Eq. 1):

$$
R_{t}=3.55 B U_{R}-185
$$

where $R_{t}$ is the rim thickness in $\mu \mathrm{m}$, and $B U_{R}$ is the rim average burnup in GWd/MTU. The rim average burnup is estimated as (Koo et al. 2001, Eq. 5):

$$
B U_{R}=1.33 B U_{\text {ave }}
$$


where $\mathrm{BU}_{\text {ave }}$ is the pellet average burnup in $\mathrm{GWd} / \mathrm{MTU}$. The bounding case shown in Figure 1.2 is given as (Koo et al. 2001, Eq. 2):

$$
R_{t}=5.28 B U_{R}-178
$$

Equation 1.3 estimates a significant rim thickness even in fuels with burnups in the range 30 GWd/MTU, which is contrary to observation. Thus, Johnson et al. (2005, Eq. 3) proposed a pessimistic expression with respect to almost all points in Figure 1.2 using the following equation:

$$
R_{t}=5.44 B U_{R}-281
$$

The calculated rim thicknesses are $193 \mu \mathrm{m}, 384 \mu \mathrm{m}$, and $298 \mu \mathrm{m}$ using Equations (1.1), (1.3), and (1.4) for a pellet average burnup of $80 \mathrm{GWd} / \mathrm{MTU}$, respectively. The above three equations are in a linear relationship, while Lassmann et al. (1995, Figure 8) suggested an exponential relationship between the burnup and rim thickness, which is also shown in Figure 1.2. The SKI Report 2005:16 (Jernkvist and Massih 2004, p.11) provided the following equation for commercial PWR fuel rods:

$$
w_{\text {Rim }}=\left\{\begin{array}{lc}
0 & E_{a v} \leq 35 \mathrm{GWd} / \mathrm{MTU} \\
0.0427\left(E_{a v}-35\right)^{2.41} & 35<E_{a v}<70 \mathrm{GWd} / \mathrm{MTU}
\end{array}\right.
$$

where $w_{\text {Rim }}$ is the width of the rim zone in $\mu \mathrm{m}$, and $E_{a v}$ is the pellet radial average burnup in GWd/MTU. At its highest valid average burnup of $70 \mathrm{GWd} / \mathrm{MTU}$, the rim thickness is about $225 \mu \mathrm{m}$. Based on the above discussion, the rim thickness should be no more than $300 \mu$ m using the pessimistic expression proposed by Johnson et al. (2005) for the HBU fuel of $80 \mathrm{GWd} / \mathrm{MTU}$, which is the highest burnup CSNF expected to be received at the Yucca Mountain repository. For a small pellet with a diameter of $0.8 \mathrm{~cm}$, a rim thickness of $300 \mu \mathrm{m}$ corresponds to approximately $15 \mathrm{vol} \%$.

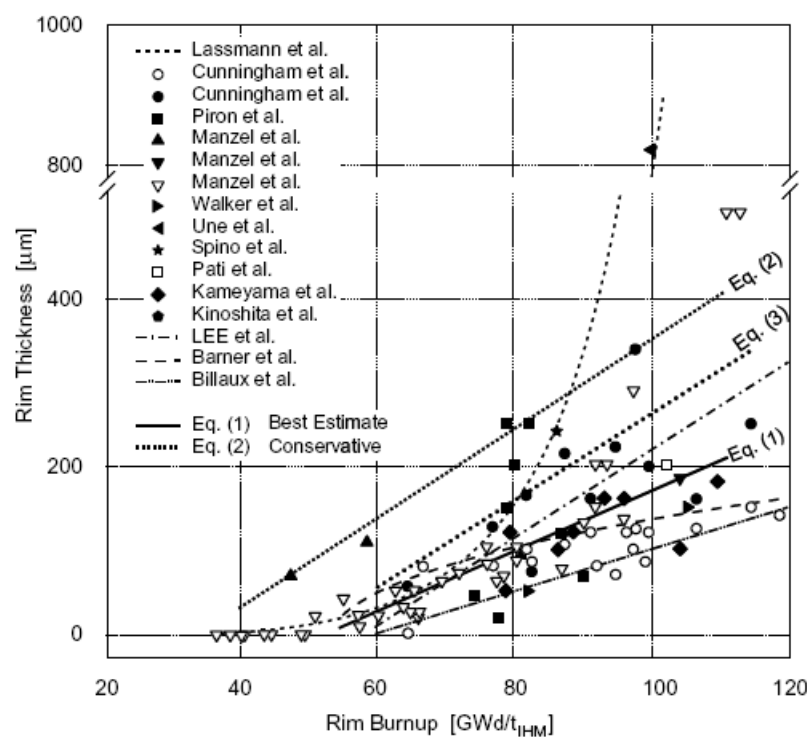

Figure 1.2. Rim Thickness as a Function of Burnup (GWd/MTU), Taken from Johnson et al. (2005, Fig. 2) 
Grain Restructuring-Based on Spino et al. (2006, 66-84), the $\mathrm{UO}_{2}$ fuel pellet is made with an initial grain size of 9 to $12 \mu \mathrm{m}$. During irradiation, grain growth occurs if the temperature is high enough, and at high burnup, the microstructure may alter to form the HBS. The grain-size distribution after irradiation in the rim between the different burnups (67 vs. $98 \mathrm{GWd} / \mathrm{MTU}$ ) is very similar, only slightly wider for the case of the HBU, as shown in the left-side figures in Figure 1.3. Comparing the grain-size distribution at different radial positions for the fuel with $98 \mathrm{GWd} / \mathrm{MTU}$ average burnup and a peak local burnup in excess of $200 \mathrm{GWD} / \mathrm{MTU}$ shows the distribution widening (grain-growth) towards the center of the pellet, as shown in the right-side figures in Figure 1.3 (Spino et al. 2006, Figure 11). It is noted that both positions $\left(\mathrm{r} / \mathrm{r}_{0}=0.7\right.$ and 0.55$)$ are not considered as the rim per Equations 1.1 through 1.4, but the grains have restructured as submicron grains nevertheless. However, the fuel examined by Spino et al. (2006) is not prototypic of fuels in that not only is the burnup much higher than any commercial fuel expected to be received at Yucca Mountain, but its ${ }^{235} \mathrm{U}$ enrichment (3.5 to 4.2\%) is relatively low compared to typical fuels meant to achieve high burnup within a few irradiation cycles and not the nine cycles for this fuel. Still, it can be concluded that as soon as local burnup exceeds the threshold for restructuring (such as $50 \mathrm{GWd} / \mathrm{MTU}$ ), the grain size decreases to the submicron range demonstrated in Figure 1.3. Spino et al. (2006) present evidence that the grain restructuring is not the only parameter characterizing the rim structure, but porosity is also important in determining the rim thickness.
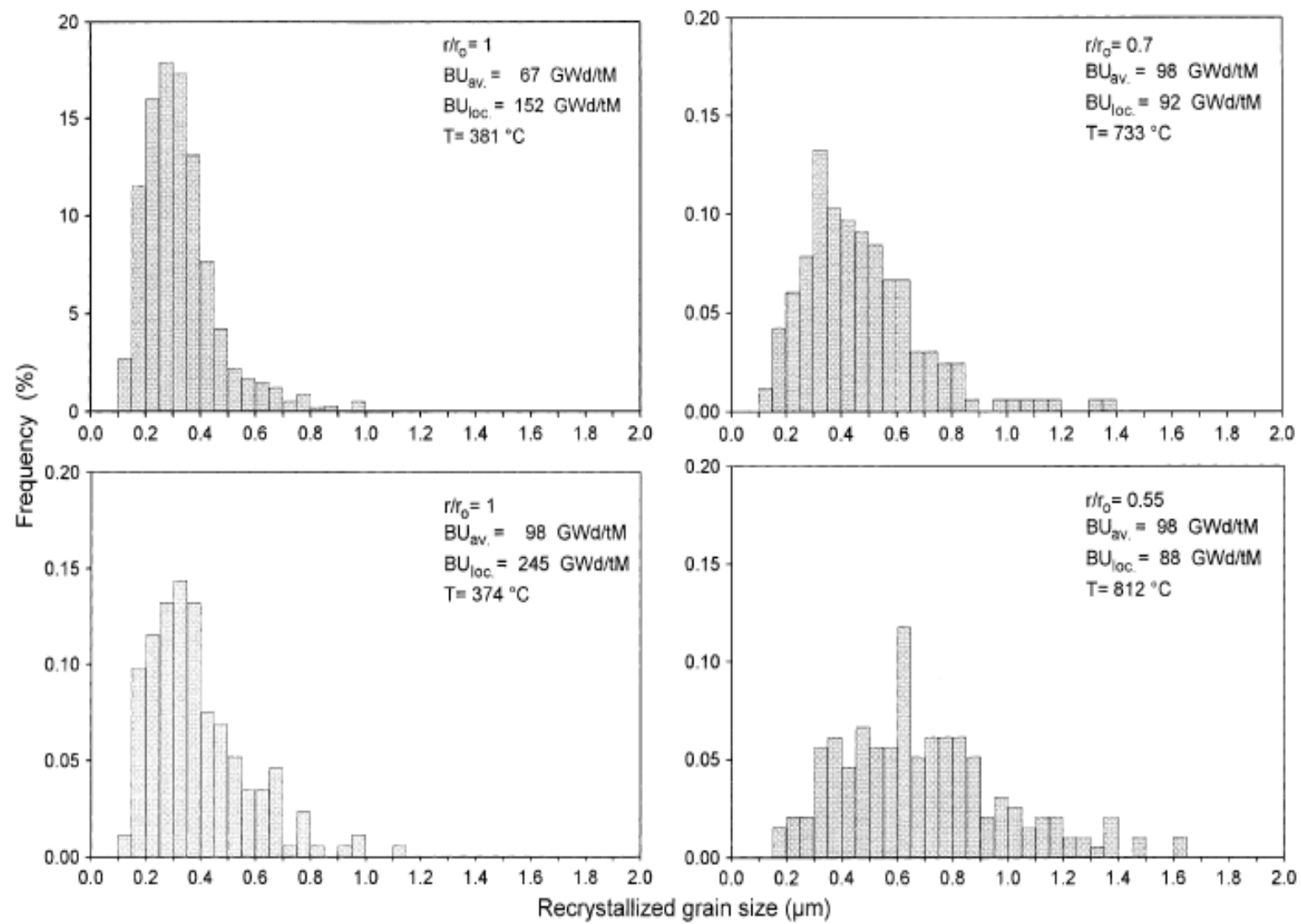

Figure 1.3. Grain-Size Distributions of Recrystallized Fuel with a Pellet-Average Burnup of $98 \mathrm{GWd} / \mathrm{MTU}$ Shown at $\mathrm{r} / \mathrm{r} 0=1,0.7$ and 0.55 . This shows a comparison with the grain-size distribution at the pellet edge of the fuel with $67 \mathrm{GWd} / \mathrm{MTU}$, taken from Spino et al. (2006, Figure 11). 
Rim Porosity - An important characteristic in the rim that differs from unaltered fuel is the porosity. Spino et al. (2006, Figure 3) provided the rim porosity as a function of the radial position for fuel with an average burnup of $98 \mathrm{GWd} / \mathrm{MTU}$, as shown in Figure 1.4. The porosity increases towards the surface of a pellet from $3 \%$ in the center to $24 \%$ in the edge. In terms of porosity as a function of local burnup, Spino et al. (2006, Figure 9) reported that the porosity increases with local burnup increase, as shown in Figure 1.5. The local burn up depends on the radial position with the highest on the edge. It can be seen from Figure 1.5 that the rim burnup can be as high as $245 \mathrm{GWd} / \mathrm{MTU}$ ( 2.5 times), compared with the average burnup of $98 \mathrm{GWd} / \mathrm{MTU}$.

In addition, Spino et al. (2006, p. 66) suggested that no clusters of interconnected pores were observed up to the maximum pore fractions checked (0.24), which was observed at the pellet surface $\left(\mathrm{r} / \mathrm{r}_{0}\right.$ $=1.00$ ) for the $98 \mathrm{GWd} / \mathrm{MTU}$ burnup. Koo et al. (2003, pp. 249-255) concluded that it is the rim porosity rather than the pore-size distribution or the rim thickness that determines the fraction of open pores connected to the pellet surface. The number of rim pores that connect with each other and form release channels in the rim increases very rapidly above the threshold porosity of around $24 \%$. If the rim porosity is less than $24 \%$, only a small number of rim pores located within $20 \mu \mathrm{m}$ from the pellet surface could contribute to gas release, resulting in a very low gas release from the rim. Once the rim porosity reaches a threshold value, extensive release channels would be developed, and considerable gas release would occur in the rim, leading to fuel rod over-pressurization and clad lift-off. The maximum rim porosity observed for $80 \mathrm{GWd} / \mathrm{MTU}$ is $14.3 \%$ (Spino et al. 2006, Table 2). This indicates that even the maximum reference SFAs ( $80 \mathrm{GWD} / \mathrm{MTU}$ ) will not reach the rim porosity high enough to form extensive release channels.

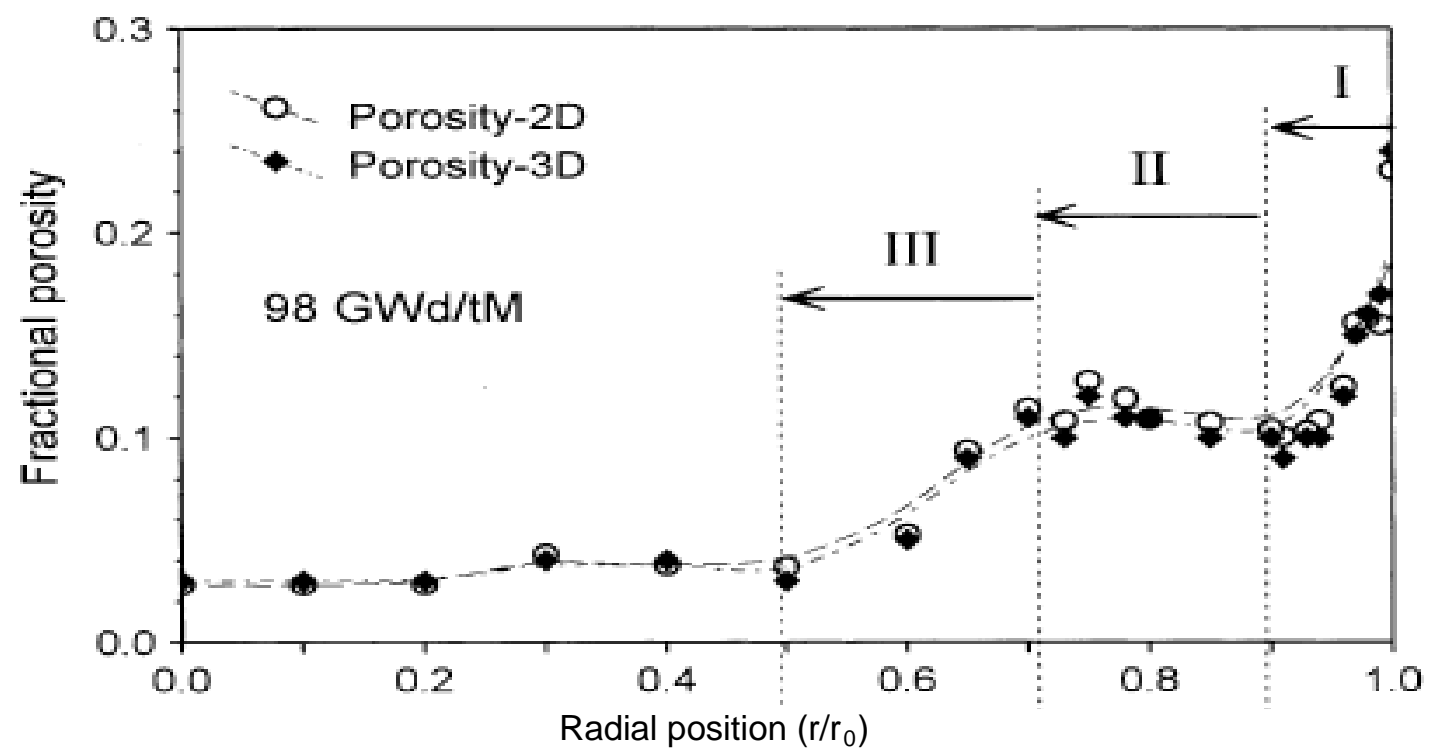

Figure 1.4. Fractional Porosity as a Function of Radial Position, Taken from Spino et al. (2006, Figure 3) 


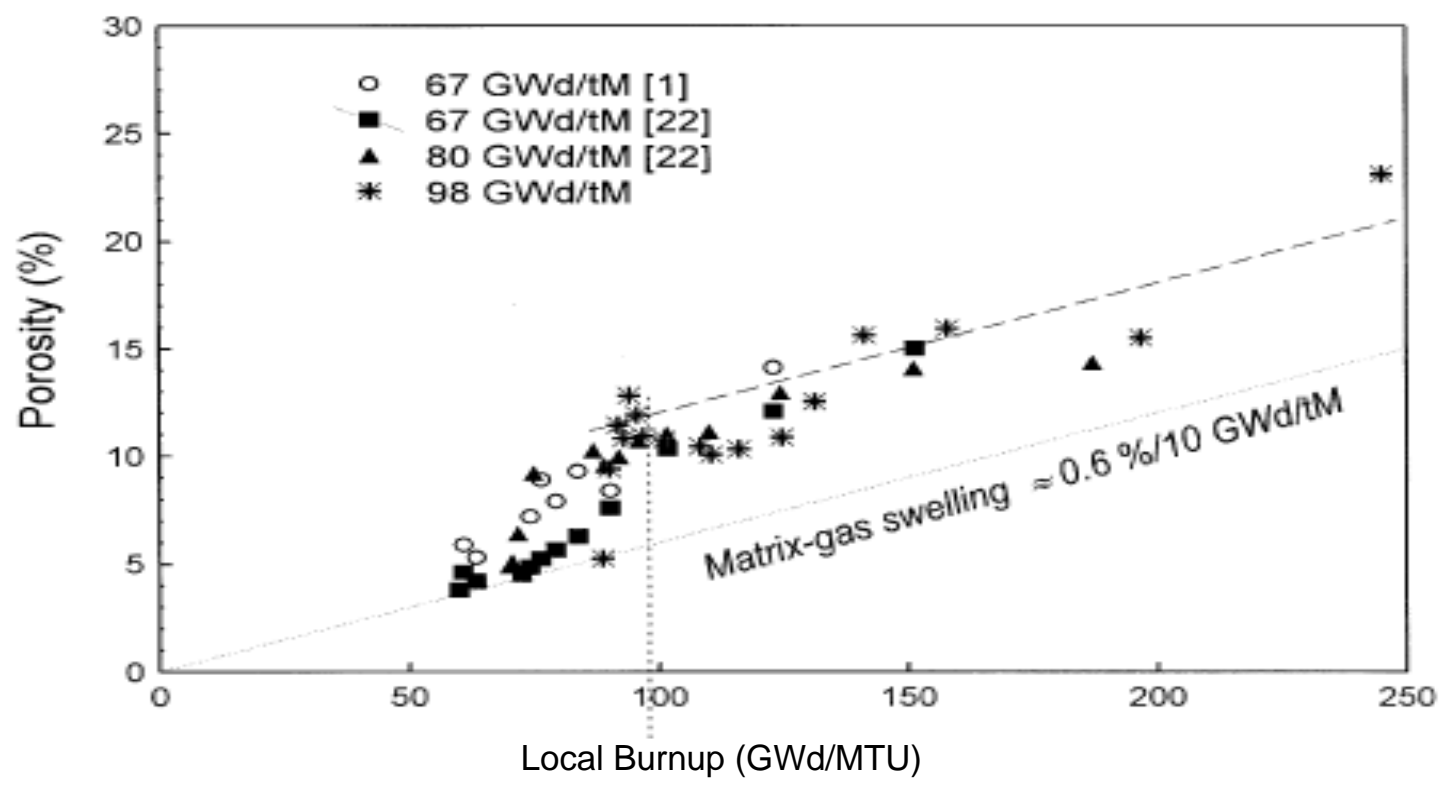

Figure 1.5. Porosity as a Function of Local Burnup, Taken from Spino et al. (2006, Figure 9)

Rim Hardness - Matzke and Spino (1997, p.176) reported that the relative hardness remains constant along most of the pellet radius and then decreases towards the fuel pellet edge where the rim structure exists, as shown in Figure 1.6. Recent testing provides the results that relative hardness decreases with fraction porosity (Spino et al. 2006, Figure 9). At a porosity of about 0.15 that corresponds to the highest porosity for an average fuel-pellet burnup of $80 \mathrm{GWd} / \mathrm{MTU}$ (see previous section), the relative hardness is about 0.7 to 0.8 , as shown in Figure 1.7. The rim hardness reduction is mainly caused by the porosity increase at the rim zone.

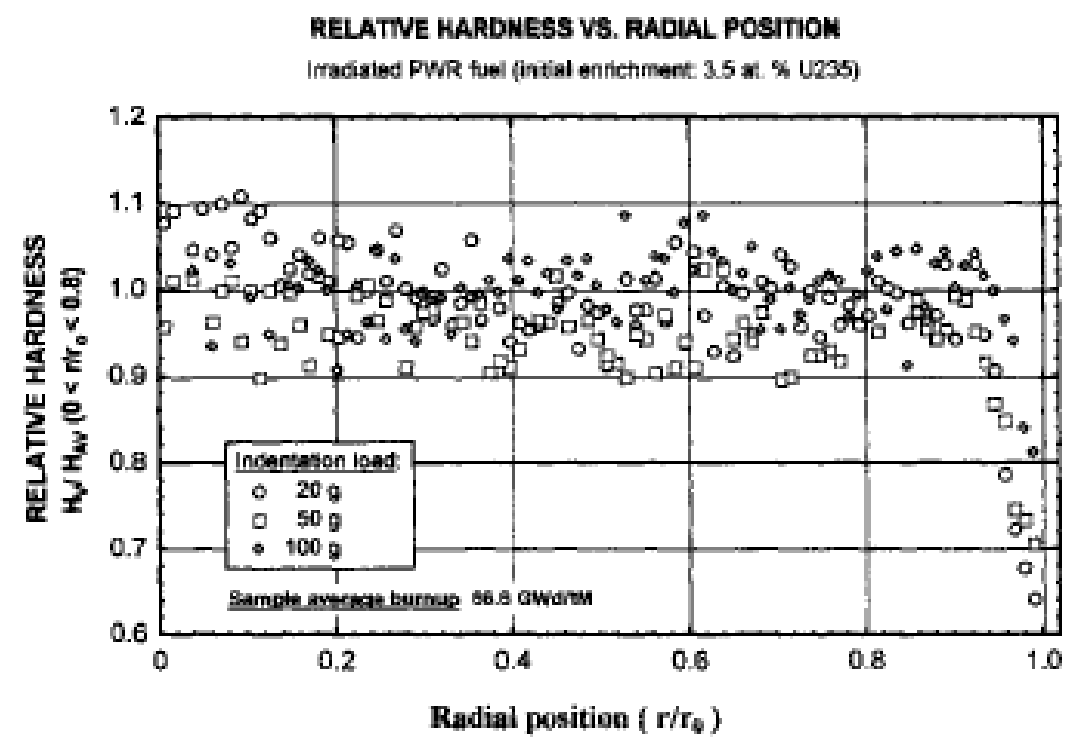

Figure 1.6. Relative Hardness as a Function of Radial Position, Taken from Matzke and Spino (1997, Figure 7) 


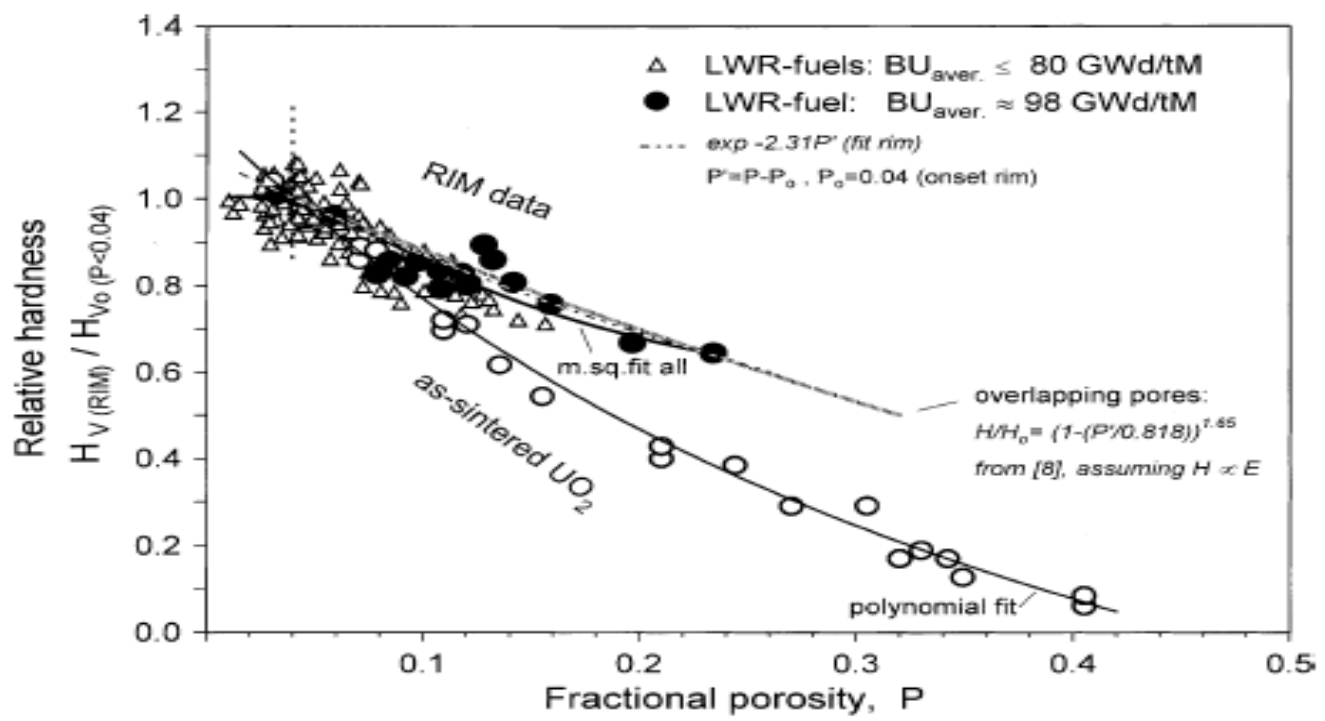

Figure 1.7. Relative Hardness as a Function of Fractional Porosity, Taken from Spino et al. (2006, Figure 9)

Rim Toughness-Matzke and Spino (1997, p.176) reported that the fracture toughness of the central parts of the fuel roughly corresponds to the range of values for unirradiated $\mathrm{UO}_{2}$, while the fracture toughness of the rim zone can be twice as large, as shown in Figure 1.8. The improvement of the fracture toughness is mainly caused by the grain refinement (Spino et al. 1996, p.186). The results indicate that although the rim contains smaller grain and more pores, it is not as easy to break into small grain particles as the unirradiated fuel. Based on the results, it seems that the release fraction of fine particles from $H B U$ fuel should not be as high as that from low burnup fuel. 
FRACTURE TOUGHNESS OF IRRADIATED PWR FUEL

Determination by the Vickers indentation method

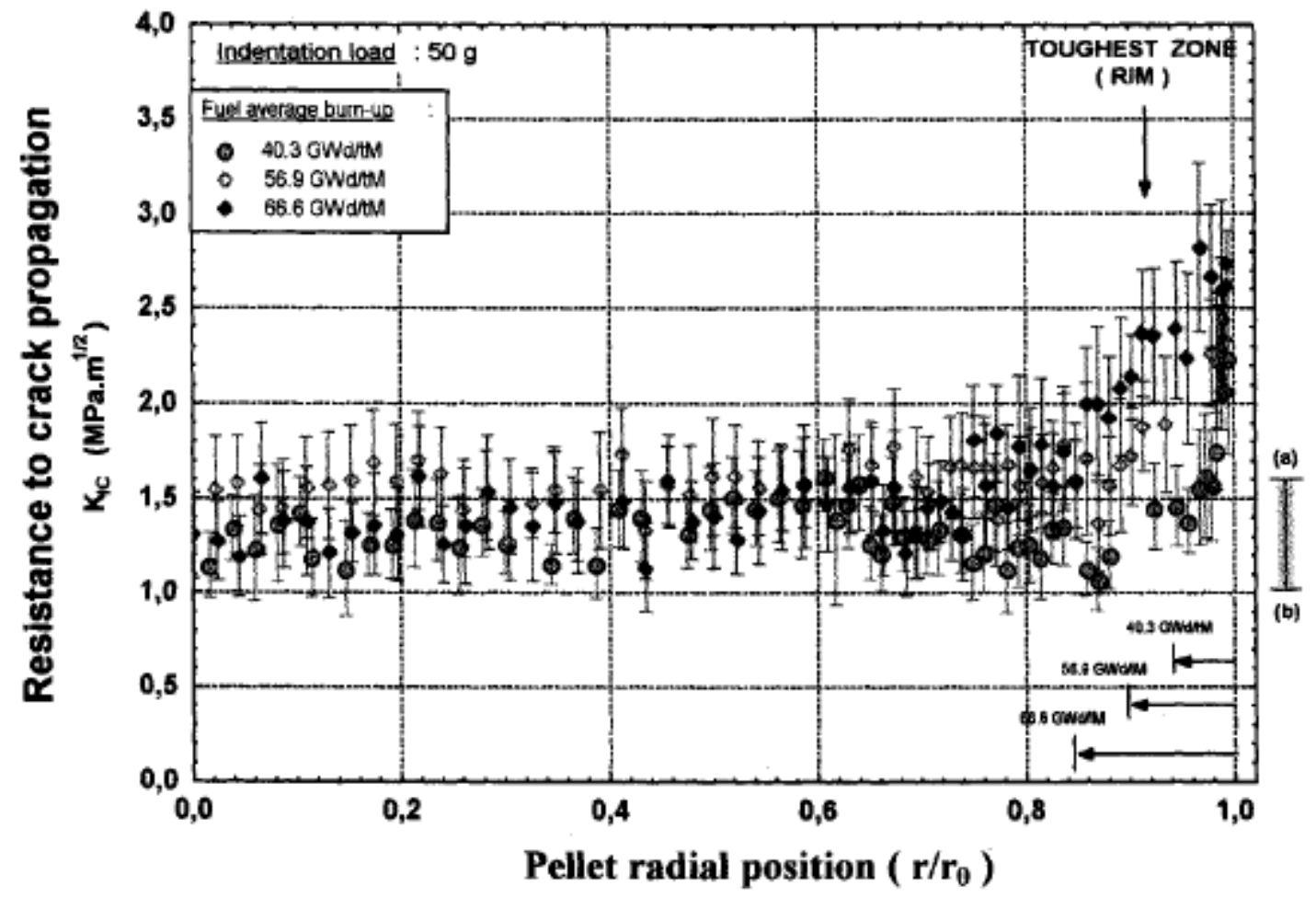

(a) $\mathrm{K}_{\mathrm{sc}}-\mathrm{UO} \mathrm{O}_{2}$ (unirradiated) - This work (low leads, plenty of lateral eracks)

(b) $\mathrm{K}_{\mathrm{NC}}-\mathrm{UO}_{2}$ (unirradiated) - Literature data (high leade, no erack branching)

Figure 1.8. Fracture Toughness as a Function of Radial Position, Taken from Matzke and Spino (1997, Figure 9)

\subsubsection{Fission-Gas Release}

Fission-gas release (FGR) was measured and modeled in a number of reports. It is a key phenomenon that must be assessed for fuel-rod design and licensing. It contributes to the fuel-rod internal pressure, with the associated risks of fuel thermal degradation and even clad failure (Bernard et al. 2002, p.125). Under the normal operation condition, the fraction of fission gas released into the gap between the fuel and cladding is only a few percent of the total fission gas produced. At low burnup and under normal operating temperatures, the rim and thermal FGR are negligible. Therefore, FGR is proportional to burnup. At high burnup, additional fission gas can be released from either a thermal effect or a rim effect or both, as shown Figure 1.9 (Bernard et al. 2002, p.128). It is reported that FGR strongly depends upon temperature, especially those experienced under reactor transient conditions.

Johnson et al. (2005, Figure 3) provided similar FGR results, as shown in Figure 1.10. The fission gas is retained in the rim pore structure, and the fission gas in this region can be considered released from the fuel matrix, even though it is not released to the void space in the fuel rod (Johnson et al. 2005, p. 60). 


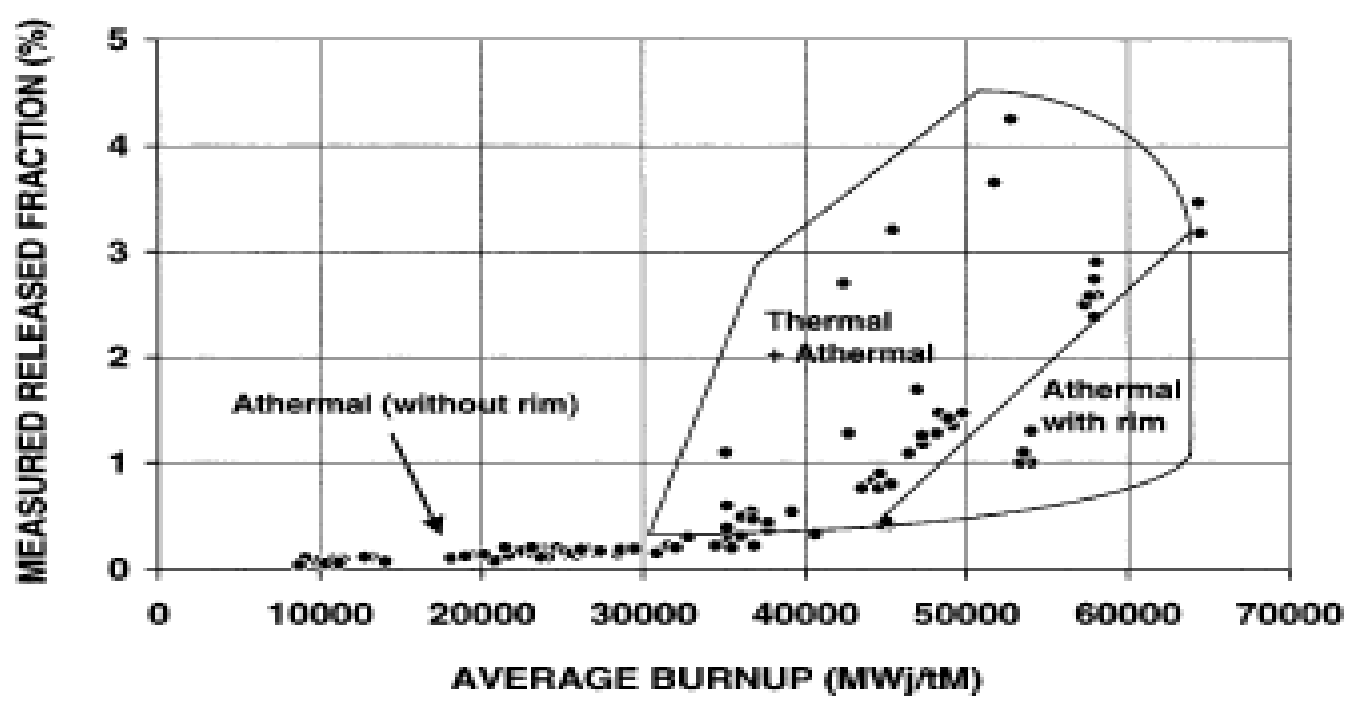

Figure 1.9. FGR as a Function of Average Burnup, Taken from Bernard et al. (2002, p.128)

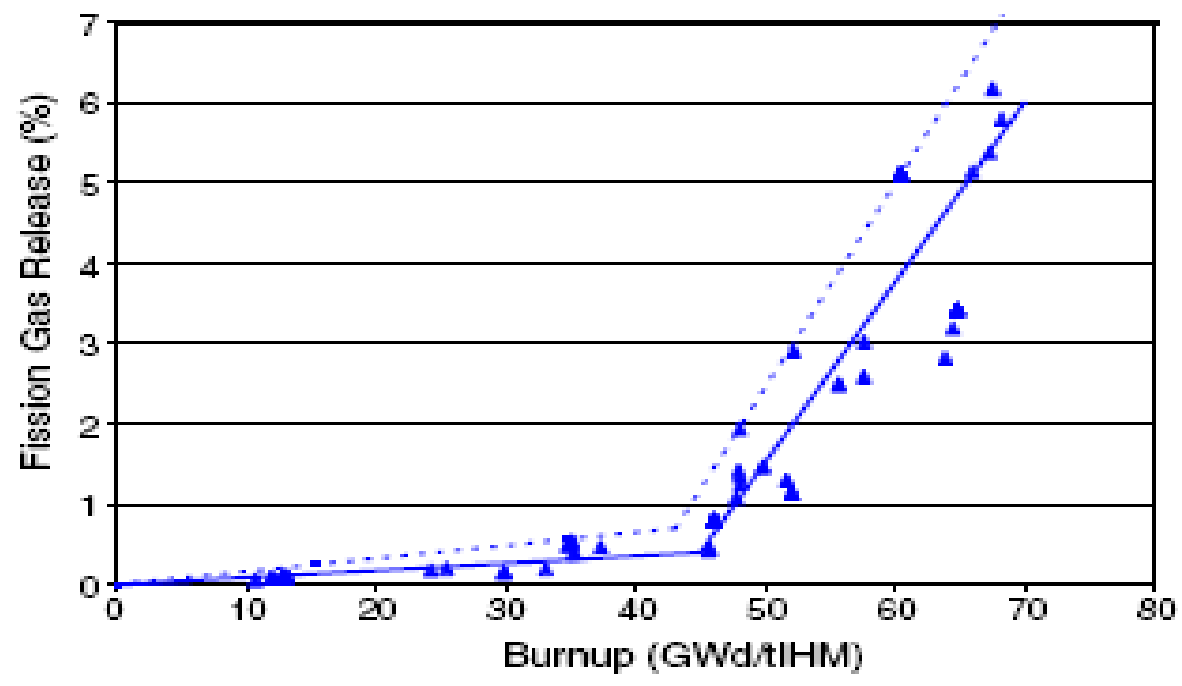

Figure 1.10. FGR from PWR Fuel as a Function of Burnup, Solid Line-Best Fit; Dashed LineBounding or Pessimistic Estimate, from Johnson et al. (2005, Fig. 3)

The FGR can be high at high temperatures, especially temperature excursions associated with reactor transients. Figure 1.11 gives the cumulative fractional releases of $\mathrm{Kr}$ from $\mathrm{UO}_{2}$ specimens irradiated in the commercial BWR ( 6 to $44 \mathrm{GWd} / \mathrm{t}$ ) and the PWR-type test reactor $(83 \mathrm{GWd} / \mathrm{t}$ ) at the ramp and isothermal annealing temperature of $1800^{\circ} \mathrm{C}$ (Une et al. 1992, p.70). However, this temperature does not occur during the preclosure repository operation.

Table 1.3 lists the FGR for the Approved Testing Material (ATM) fuels characterized and used by the project. The highest FGR from any of the rods, including a rod with a rod average burnup of 
$61 \mathrm{GWd} / \mathrm{MTU}$, was 18\%. Manzel and Coquerelle (1997, Figure 1) reported an FGR of approximately $12 \%$ for a PWR fuel rod with a rod average burnup of $78 \mathrm{GWd} / \mathrm{MTU}$ and Van Swam et al. (2007,

Table 1) examined 35 PWR rods with burnups between 70 and $73 \mathrm{GWd} / \mathrm{MTU}$ and found all FGRs to be less than or equal to 7\%. They also examined BWR fuel rods and found that in all cases except for fuels with very large initial pellet-to-clad gaps, the FGR was less than or equal to $12 \%$. The rods with the large initial gap exhibited FGRs as high as $27.9 \%$. In all cases, the FGR is less than the conservative estimate of $30 \%$ used by Anderson et al. (1996, p. 30) in NUREG/CR-6487.

It is concluded that typically only a few percent of the total fission gas is available for release into the environment in the event of a drop and breach of the fuel cladding. For fuel in a canister (e.g., TAD, cask, dual purpose canister, etc.), the canister must also be breached for the fission gas to be released to the environment.

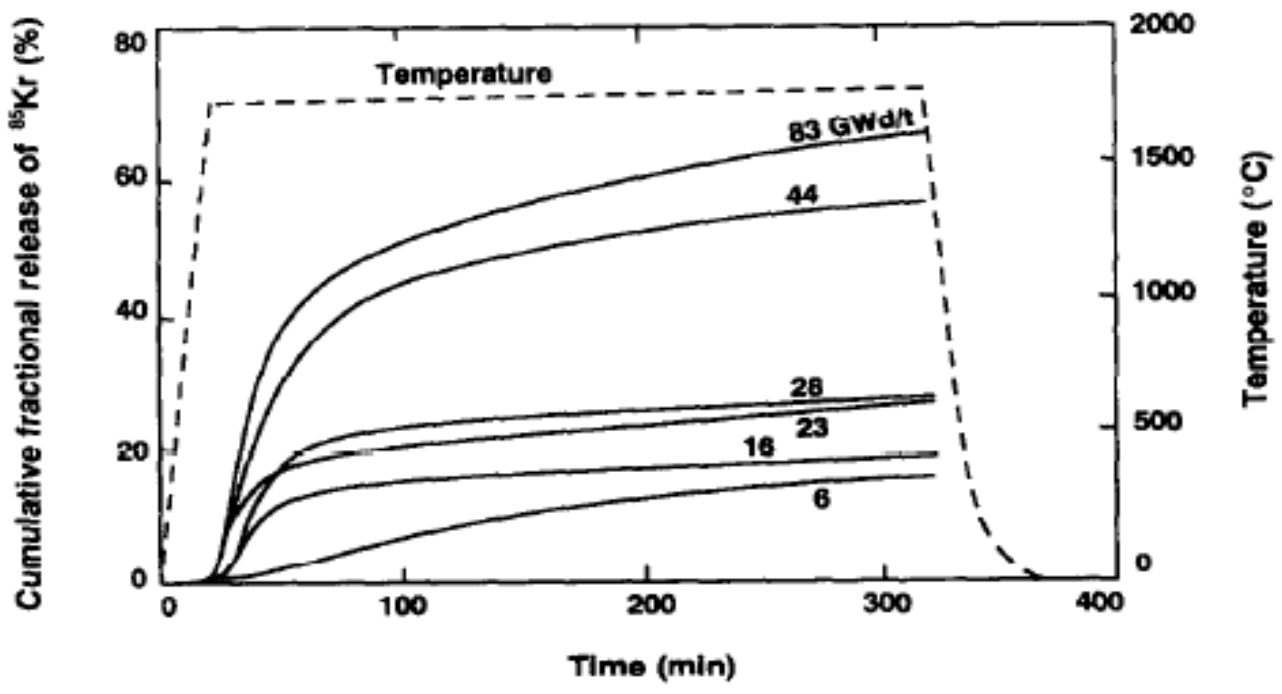

Figure 1.11. Cumulative Fractional Release of $\mathrm{Kr}$ at the Ramp and Isothermal Annealing Temperature of $1800^{\circ} \mathrm{C}$ from Une et al. (1992, Figure 5) 
Table 1.3. Fission Gas Release from ATM Fuels

\begin{tabular}{|c|c|c|c|}
\hline ATM & $\begin{array}{c}\text { Rod } \\
\text { Identification }\end{array}$ & $\begin{array}{c}\text { Rod Average Burnup } \\
\text { (GWd/MTU) }\end{array}$ & Fission Gas Release \% \\
\hline ATM-103 ${ }^{(\mathrm{a})}$ & MLA098 & 29.8 & 0.25 \\
\hline \multirow[t]{3}{*}{ ATM-104 ${ }^{(\mathrm{b})}$} & MKP028 & 40.26 & 0.38 \\
\hline & MKP059 & 42.56 & 0.62 \\
\hline & MKP109 & 40.40 & 1.10 \\
\hline \multirow[t]{2}{*}{ ATM-105 ${ }^{(\mathrm{c})}$} & ADD2966 & 28.05 & 7.85 \\
\hline & ADD2974 & 28.05 & 0.59 \\
\hline \multirow[t]{3}{*}{ ATM-106 ${ }^{(\mathrm{d})}$} & NBD040 & 42.32 & 1.4 \\
\hline & NBD095 & 42.32 & 7.4 \\
\hline & NBD107 & 42.32 & 11.2 \\
\hline ATM-106 ${ }^{(\mathrm{e})}$ & NBD131 & 46 & 18 \\
\hline \multirow[t]{3}{*}{ ATM-109 ${ }^{(f)}$} & $\mathrm{A} 2$ & 60.6 & 4.42 \\
\hline & G3 & 61.1 & 2.95 \\
\hline & E7 & 52.2 & 3.52 \\
\hline \multicolumn{4}{|c|}{ (a) Guenther et al. (1988a, Table 4.7) } \\
\hline \multicolumn{4}{|c|}{ (b) Guenther et al. (1991a, Table 8.7) } \\
\hline \multicolumn{4}{|c|}{ (c) Guenther et al. (1991b, Table 4.10) note that burnup is bundle average } \\
\hline \multicolumn{4}{|c|}{ (d) Guenther et al. (1988b, Table 4.10) } \\
\hline \multicolumn{4}{|c|}{ (e) Guenther et al. (1994, Table 4.4) note that burnup and FGR are estimates } \\
\hline \multicolumn{4}{|c|}{ (f) Vaidyanathan et al. (1997, Table 2) } \\
\hline
\end{tabular}

\subsubsection{Fission Volatile Radionuclide Release}

The main contributors to FGR are the fission products $\mathrm{Xe}$ and $\mathrm{Kr}$ since they are gases, highly mobile, and do not react with other elements. Other elements such as cesium, iodine, and tellurium, and to a lesser extent, barium, ruthenium, and technetium, are insoluble in the $\mathrm{UO}_{2}$ lattice and tend to be mobile at the high temperatures during reactor operation. However, unlike the rare gases, these other elements tend to react with the fuel or other elements within the matrix that limits their release from the fuel pellet. Still, a fraction of these elements that may be volatile at the temperatures of interest for fuel handling transport out of the fuel grains, first to the grain boundaries and then to the fuel-cladding gap. That fraction within the gap may be available for release along with the fission gases upon breach of the cladding. The grain-boundary fraction would be available for release only after the grain boundaries open because of oxidation. Table 1.4 lists the gap and grain boundary inventories measured by leaching for some of the fuels in Table 1.3. From Table 1.4, it is clear that only minimal quantities of the volatile radionuclides, with the exception of iodine, are located outside of the matrix. Thus, it is appropriate that the $A R F$ for the volatile elements is similar to that for fine particles of fuel, with the exception of iodine and sometimes cesium, which are similar to the FGR. The respirable fraction for the volatile elements is similar to that for gas. 
Table 1.4. Gap and Grain-Boundary Inventories

\begin{tabular}{|c|c|c|c|c|c|c|c|c|c|c|c|c|}
\hline ATM & $\operatorname{Rod} \#$ & Segment & $\begin{array}{l}\text { Local Burnup } \\
\text { (GWd/MTU) }\end{array}$ & $\begin{array}{c}\text { Fission Gas } \\
\text { Release } \\
(\%)\end{array}$ & $\begin{array}{c}\text { Cs-GI } \\
(\%)\end{array}$ & $\begin{array}{c}\text { Cs-GBI } \\
(\%)\end{array}$ & $\begin{array}{c}\text { Tc-GI } \\
(\%)\end{array}$ & $\begin{array}{c}\text { Tc-GBI } \\
(\%)\end{array}$ & $\begin{array}{l}\text { Sr-GI } \\
(\%)\end{array}$ & $\begin{array}{l}\text { Sr-GBI } \\
(\%)\end{array}$ & $\begin{array}{l}\text { I-GI } \\
(\%)\end{array}$ & $\begin{array}{c}\text { I-GBI } \\
(\%)\end{array}$ \\
\hline 104 & MKP-109 & $\mathrm{X}$ & 44 & 1.1 & $1.18 \mathrm{E}+00$ & $1.98 \mathrm{E}-01$ & & $5.04 \mathrm{E}-02$ & $3.97 \mathrm{E}-02$ & $2.14 \mathrm{E}-02$ & & \\
\hline \multirow[t]{5}{*}{105} & ADD-2966 & $\mathrm{AA}$ & 34 & 7.85 & $7.21 \mathrm{E}-01$ & $1.17 \mathrm{E}+00$ & $3.44 \mathrm{E}-05$ & & $8.70 \mathrm{E}-03$ & $2.38 \mathrm{E}-02$ & & \\
\hline & & $\mathrm{H}$ & 33 & 7.85 & $8.02 \mathrm{E}-01$ & $3.67 \mathrm{E}-01$ & & $4.76 \mathrm{E}-02$ & $3.68 \mathrm{E}-02$ & $5.75 \mathrm{E}-02$ & & \\
\hline & & $\mathrm{Q}$ & 34 & 7.85 & $2.23 \mathrm{E}+00$ & & & & & & $1.52 \mathrm{E}+00$ & \\
\hline & & $\mathrm{Q}$ & 34 & 7.85 & $1.55 \mathrm{E}+00$ & & & & & & $3.28 \mathrm{E}+00$ & \\
\hline & & $\mathrm{Q}$ & 34 & 7.85 & & $6.24 \mathrm{E}-01$ & & & & & & $5.15 \mathrm{E}+00$ \\
\hline \multirow[t]{3}{*}{105} & ADD-2974 & BB & 31 & 0.59 & $2.07 \mathrm{E}-01$ & $1.88 \mathrm{E}-01$ & & $6.63 \mathrm{E}-02$ & $1.97 \mathrm{E}-02$ & $8.00 \mathrm{E}-02$ & $1.68 \mathrm{E}-02$ & \\
\hline & & $\mathrm{BB}$ & 31 & 0.59 & $2.78 \mathrm{E}-01$ & & & & & & $1.15 \mathrm{E}-01$ & \\
\hline & & $\mathrm{BB}$ & 31 & 0.59 & & 7.37E-01 & & & & & & $2.09 \mathrm{E}+00$ \\
\hline \multirow[t]{4}{*}{106} & NBD-095 & $\mathrm{DD}$ & 45.8 & 7.4 & $1.80 \mathrm{E}+00$ & & & & & & $9.37 \mathrm{E}-02$ & \\
\hline & & DD & 45.8 & 7.4 & & $6.96 \mathrm{E}-01$ & & & & & & $7.98 \mathrm{E}+00$ \\
\hline & & DD & 45.8 & 7.4 & & $6.48 \mathrm{E}-01$ & & & & & & $7.53 \mathrm{E}+00$ \\
\hline & & & & & & & & & & & & \\
\hline \multirow[t]{6}{*}{106} & NBD-107 & $\mathrm{N}$ & 46.5 & 11.2 & $2.57 \mathrm{E}+00$ & $1.00 \mathrm{E}+00$ & $1.51 \mathrm{E}-02$ & $1.54 \mathrm{E}-02$ & $2.30 \mathrm{E}-02$ & $1.34 \mathrm{E}-01$ & & \\
\hline & & $\mathrm{N}$ & 46.5 & 11.2 & $2.32 \mathrm{E}+00$ & & $8.22 \mathrm{E}-03$ & & $1.22 \mathrm{E}-02$ & & & \\
\hline & & DD & 46.6 & 11.2 & $3.21 \mathrm{E}+00$ & & & & & & $1.44 \mathrm{E}+00$ & \\
\hline & & DD & 46.6 & 11.2 & $3.00 \mathrm{E}+00$ & & & & & & $1.08 \mathrm{E}+00$ & \\
\hline & & DD & 46.6 & 11.2 & & 7.64E-01 & & & & & & $7.48 \mathrm{E}+00$ \\
\hline & & DD & 46.6 & 11.2 & & $8.14 \mathrm{E}-01$ & & & & & & $9.13 \mathrm{E}+00$ \\
\hline \multirow{6}{*}{106} & NBD-131 & $\mathrm{O}$ & 48.1 & 18 & $4.25 \mathrm{E}+00$ & $9.34 \mathrm{E}-01$ & $5.42 \mathrm{E}-02$ & $1.33 \mathrm{E}-01$ & $9.70 \mathrm{E}-02$ & $8.65 \mathrm{E}-02$ & & \\
\hline & & $\mathrm{O}$ & 48.1 & 18 & $5.05 \mathrm{E}+00$ & & $2.55 \mathrm{E}-02$ & & $4.08 \mathrm{E}-02$ & & & \\
\hline & & $\mathrm{Y}$ & 47.5 & 18 & $7.46 \mathrm{E}+00$ & & & & & & $1.82 \mathrm{E}+01$ & \\
\hline & & $\mathrm{Y}$ & 47.5 & 18 & $1.05 \mathrm{E}+01$ & & & & & & $1.23 \mathrm{E}+01$ & \\
\hline & & $\mathrm{Y}$ & 47.5 & 18 & & $1.10 \mathrm{E}+00$ & & & & & & $8.32 \mathrm{E}+00$ \\
\hline & & $\mathrm{Y}$ & 47.5 & 18 & & $1.23 \mathrm{E}+00$ & & & & & & $8.54 \mathrm{E}+00$ \\
\hline
\end{tabular}

GI is the gap inventory; GBI is the grain-boundary inventory. 


\subsection{CSNF Oxidation}

The temperature to which CSNF is exposed to an oxidizing atmosphere is the most important parameter when determining the possibility and extent of oxidation, which in turn affects the quantity and size distribution of fuel available for release from a breached rod. It is necessary for the cladding to be breached for oxidation and radionuclide release to occur. For the purposes of this report, it is assumed that the maximum time a breached rod may be exposed to an oxidizing atmosphere is 720 hours (30 days) (NRC 2008) after which mitigating steps (e.g., canning of the fuel, sealing of the affected canister, etc.) will prevent further oxidation and radionuclide release. Other factors affecting the oxidation kinetics of the fuel, such as grain size and burnup, are discussed in this section. Finally, the results of fragment oxidation tests performed during Fiscal Year 2007 are presented.

\subsection{Temperature Effects}

\subsection{1 $\mathrm{UO}_{2} \Rightarrow \mathrm{UO}_{2.4}$}

For almost all fuels of interest, it has been shown that fuel oxidation will occur by first oxidizing the cubic $\mathrm{UO}_{2}$ phase to a cubic phase resembling $\mathrm{U}_{4} \mathrm{O}_{9}$, but in a hyperstoichiometric form, it is often represented as either $\mathrm{U}_{4} \mathrm{O}_{9+\mathrm{x}}$ or $\mathrm{UO}_{2.4}$. This transition has been shown to be relatively independent of burnup (Hanson 1998; McEachern and Taylor 1998; CRWMS 2000). Although full conversion to $\mathrm{UO}_{2.4}$ is dependent on the initial grain size (Kansa et al. 1998) with small-grained fuels oxidizing faster, the variation for typical fuels is considered within the uncertainties of the experimental data. Using the equation for the nominal case from CRWMS (2000), we obtain

$$
\mathrm{t}_{2.4}(\mathrm{~h})=1.40 \times 10^{-8} \exp (105 \mathrm{~kJ} / \mathrm{RT})
$$

where $\mathrm{t}_{2.4}$ is the time required to reach full conversion to $\mathrm{UO}_{2.4}, \mathrm{R}$ is the universal gas constant (8.314 $\left.\mathrm{J} \mathrm{mol}^{-1} \mathrm{~K}^{-1}\right)$, and $\mathrm{T}$ is the temperature $\left(\mathrm{K}=273+\mathrm{T}\left[{ }^{\circ} \mathrm{C}\right]\right)$. Thus, if the temperature of the fuel can be maintained at or below about $240^{\circ} \mathrm{C}$, then during the 720 hours of exposure, only oxidation to $\mathrm{UO}_{2.4}$ will occur. (This applies to fuel with burnups greater than about $15 \mathrm{GWd} / \mathrm{MTU}$ because Novak et al. [1983] showed severe cladding splitting within 200 hours at $250^{\circ} \mathrm{C}$ for CANDU fuel with a burnup of approximately $8 \mathrm{GWd}$ /MTU.) Since this oxidation is accompanied by a slight contraction of the fuel, no swelling and no additional cladding unzipping will occur. However, fission gasses and volatiles located within the gap and grain boundaries, as shown in Table 1.4, may be released. Similarly, the oxidized grain boundaries may allow fuel relocation of as-irradiated grain-sized particles. This relocation is based upon the observation that fuel with oxidized grain boundaries tends to be friable and can break apart upon handling. Any relocation will be dependent on the cladding defect size and the movement of the damaged rod.

It is important to note that the work of Einziger and Strain (1986, Figure 10) seems to indicate that significant fuel oxidation and cladding splitting can occur in under 130 hours at $235^{\circ} \mathrm{C}$. However, since the title of the paper refers to temperatures between 250 and $360^{\circ} \mathrm{C}$, it is clear from the data presented in Table IV of Einziger and Strain (1986, p. 91) and the more detailed report of the work referenced in EPRI (1986 p. 3-20) that the caption for picture b in Figure 10 of Einziger and Strain (1986) has a typographical 
error, and the temperature should indeed be $325^{\circ} \mathrm{C}$. There is therefore no contradiction to Equation 2.1, and a maximum fuel temperature of $240^{\circ} \mathrm{C}$ would indeed prevent additional cladding splitting.

Finally, some have hypothesized that the restructured rim, or HBS, may lead to more rapid oxidation than that proposed in Equation 2.1 because of the extremely fine-grained material and high porosity (see Section 1.1.1). Only minimal oxidation testing has been performed on CSNF with burnups high enough for HBS to exist.

Previously unpublished data from ATM-109 fuel oxidized at $305^{\circ} \mathrm{C}$ in comparison with other fuels oxidized at the same temperature (see, e.g., Hanson 1998) shows that even for a fragment from the rim containing HBS, the time to reach the plateau (see Section 2.2 but assumed to be the $\mathrm{UO}_{2.4}$ phase) was approximately 26 hours. Although this time is less than the 43 hours predicted by Equation 2.1, it is important to stress the variability of the data (CRWMS 2000, Table 1 and Figure 5) used to generate the equation. This variability is a result of differing grain sizes and the presence of hydrated phases for some fuels. While the time to reach the plateau for the fragment of the same fuel but from the center of the pellet was approximately 70 hours, it is not possible to attribute the difference solely to the presence of the HBS, especially because the weight-gain curves were obviously skewed from the release of fission gasses during the oxidation process (see Figure 2.1), which was much more pronounced for the fragment from the rim. Even if one assumes the HBS to be responsible for this observed difference, compared to other fuels oxidized at the same temperature, the effect of the HBS is negligible, and there is no evidence of the HBS resulting in significantly faster oxidation.

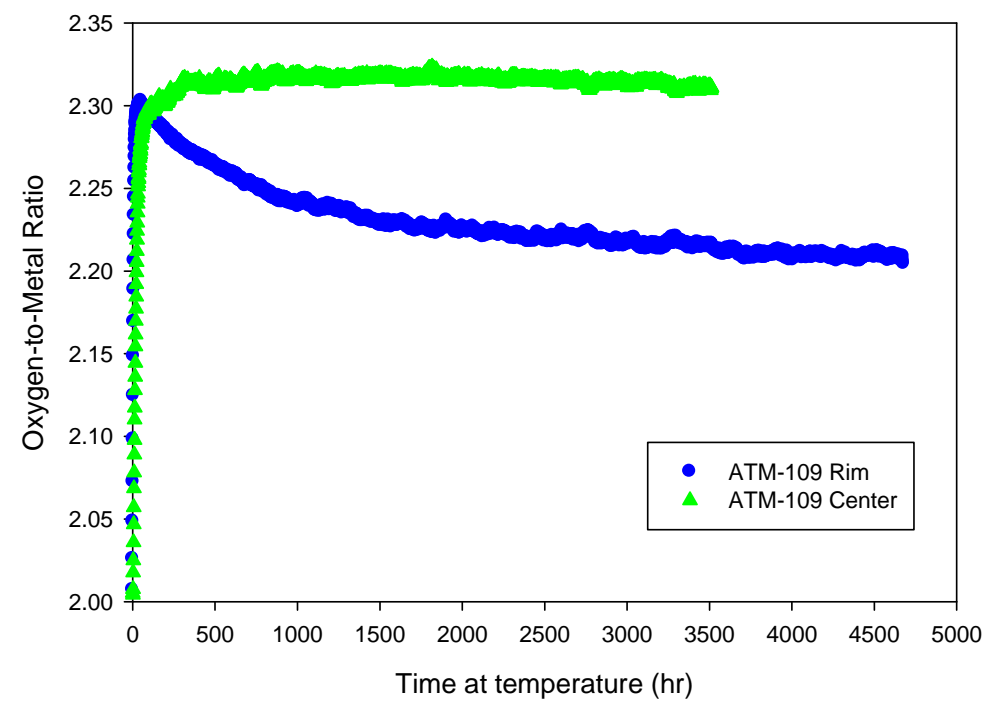

Figure 2.1. Oxidation of $\mathrm{HBU}$ ATM-109 Fuel at $305^{\circ} \mathrm{C}$

A recent paper by Colle et al. (2006) plotted the oxidation curve of spent fuel irradiated to 65 GWd/MTU (approximately the same as the ATM-109 fuel shown in Figure 2.2 and tested by PNNL during Fiscal Year 2007) at $670 \mathrm{~K}\left(397^{\circ} \mathrm{C}\right)$ as shown in Figure 2.2. Assuming that the plateau was the last data point $(\mathrm{O} / \mathrm{U} 2.25)$ before observing the orthorhombic phase, the $\mathrm{t}_{2.4}$ is approximately 2 hours, the same as predicted by Equation 2.1. Colle et al. (2006) show that at temperatures approaching $400^{\circ} \mathrm{C}$, there is complete oxidation to form $\mathrm{U}_{3} \mathrm{O}_{8}$ within about 2 days. However, it is unclear what was meant in 
their paper when they stated (p. 231) that "Samples were taken from pellets, where the in-pile temperature was low enough to maintain all fission products homogeneously dispersed or frozen in the lattice." If the sample used for oxidation came from the lower end of the fuel rod, then it is possible that the burnup of the sample could be much lower than the rod-average $65 \mathrm{GWd} / \mathrm{MTU}$ reported.

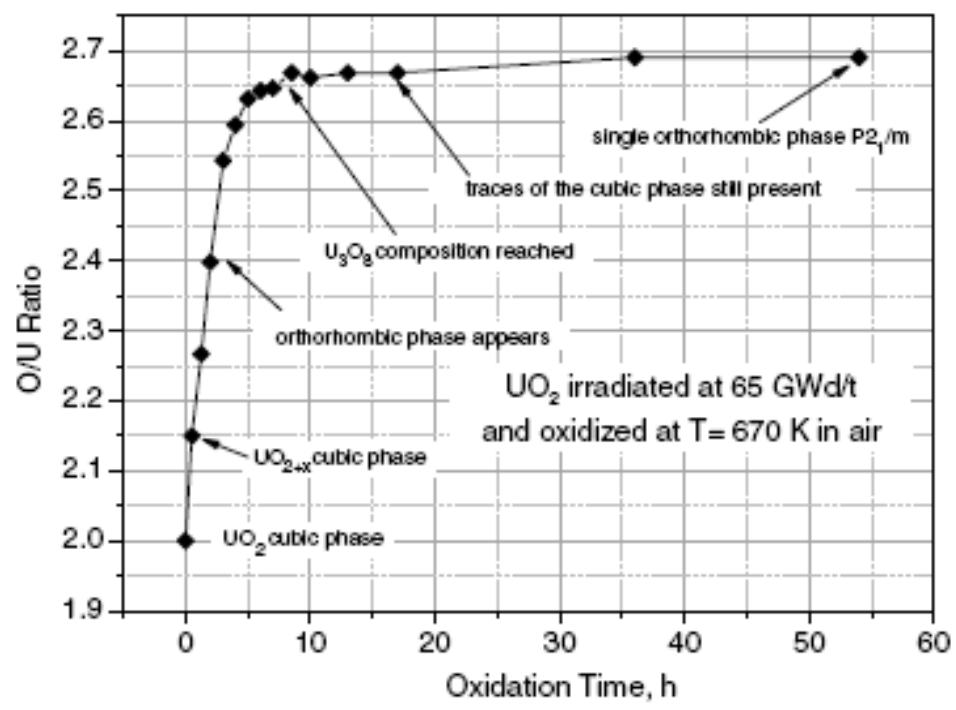

Figure 2.2. Oxidation Curve of CSNF Irradiate to $65 \mathrm{GWd} / \mathrm{MTU}$ and Oxidized at $397^{\circ} \mathrm{C}$, from Colle et al. (2006, Figure 3)

\subsection{2 $\mathrm{UO}_{2.4} \Rightarrow \mathrm{U}_{3} \mathrm{O}_{8}$}

After an individual grain oxidizes to $\mathrm{UO}_{2.4}$, it then oxidizes to $\mathrm{U}_{3} \mathrm{O}_{8} \cdot \mathrm{U}_{3} \mathrm{O}_{8}$ is less dense than the original $\mathrm{UO}_{2}$, and as it forms, it exerts a hoop stress on the cladding that can propagate the original defect and result in cladding unzipping. $\mathrm{U}_{3} \mathrm{O}_{8}$ also spalls from the sample as it forms and is available for relocation. At low temperatures and with $\mathrm{HBU}$, an extended plateau exists where there is no appreciable weight gain. It is unclear what processes are occurring during the plateau, but the orthorhombic $\mathrm{U}_{3} \mathrm{O}_{8}$ phase is not observed until the weight gain continues past this plateau. The incubation time defined in CRWMS 2000 is $t_{2.4}$ plus the time required for enough $\mathrm{U}_{3} \mathrm{O}_{8}$ to form to start propagating the cladding defect. This incubation time, $t_{\text {inc }}$, is expressed as

$$
\mathrm{t}_{\text {inc }}=\mathrm{t}_{2.4}+\lambda_{\text {inc }} \mathrm{k}_{75} \exp [(150 \mathrm{~kJ}+\alpha \times \text { Burnup }) / \mathrm{RT}]
$$

where $\quad t_{\text {inc }}=$ hours

$\mathrm{t}_{2.4}=$ that given by equation 2.1

$\lambda_{\text {inc }}=$ a parameter specified in CRWMS 2000 and related to the fuel/clad gap and the strain required for defect propagation

$\mathrm{k}_{75}=4.84 \times 10^{-14}$ for the nominal case or $1.48 \times 10^{-14}$ for the bounding case

$\alpha=1.0 \mathrm{~kJ} \mathrm{~mol}^{-1}$ per GWd/MTU local burnup of the fuel in GWd/MTU 
burnup

CRWMS (2000) assumed the very conservative case of no initial fuel/clad gap and zero strain needed for crack propagation so that $\lambda_{\text {inc }}$ is 0.0063 . This factor can increase by almost a factor of 10 (with corresponding delay in the incubation time) if the contraction of the fuel as it oxidizes to $\mathrm{UO}_{2.4}$, the initial fuel/clad gap (which will only exist for low to moderate burnup fuels), and realistic strains of $1 \%$ or $2 \%$ are included.

Ferry et al. (2005, p. 189) reported the incubation times as a function of temperature (K) for a number of cases:

$$
\begin{array}{ll}
\mathrm{t}_{\text {inc }}(\mathrm{h})=5.6 \times 10^{-6} \exp (9140 / \mathrm{T}) & \text { CANDU fuel } \\
\mathrm{t}_{\text {inc }}(\mathrm{h})=6.2 \times 10^{-9} \exp (13245 / \mathrm{T}) & \text { PWR/BWR fuels } \\
\mathrm{t}_{\text {inc }}(\mathrm{h})=1.692 \times 10^{-8} \exp (13648 / \mathrm{T}) & \text { PWR small defects } \\
\mathrm{t}_{\text {inc }}(\mathrm{h})=3.198 \times 10^{-11} \exp (16774 / \mathrm{T}) & \text { PWR large defects } \\
\mathrm{t}_{\text {inc }}(\mathrm{h})=5.817 \times 10^{-10} \exp (15349 / \mathrm{T}) & \text { PWR all defects }
\end{array}
$$

Most of these fits were obtained using the data from Einziger and Strain (1986), so the PWR fuel had a burnup of only $27 \mathrm{GWd} / \mathrm{MTU}$. While showing the clear dependence on burnup (i.e., the marked difference in the activation energy between the CANDU fuels and the LWR fuels) and on defect size, it is still clear that even at $300^{\circ} \mathrm{C}$, the incubation time for these lower burnup fuels is much less than the 720 hours assumed for this analysis.

Equations 2.1 through 2.7 were developed using fuel samples with burnups below about 40 $\mathrm{GWd} / \mathrm{MTU}$ and maximum temperatures of $350^{\circ} \mathrm{C}$. Extrapolating these equations to higher burnups and temperatures may not be warranted. Colle et al. (2006) reported that spent fuel with a burnup of 65 $\mathrm{GWd} / \mathrm{MTU}$ essentially fully converted to $\mathrm{U}_{3} \mathrm{O}_{8}$ in about 10 hours at $670 \mathrm{~K}\left(397^{\circ} \mathrm{C}\right)$, as seen in Figure 2.3 . This is substantially faster than the approximately 2800 hours predicted by the nominal model in CRWMS (2000). As discussed in Section 2.1.1, however, this may be because the actual sample oxidized by Colle et al. (2006) had a lower burnup than the $65 \mathrm{GWd} / \mathrm{MTU}$ reported for the rod average.

\subsection{Grain Size}

CSNF is known to oxidize by rapid attack of the grain boundaries followed by oxidation of the individual grains. For the $\mathrm{UO}_{2}$ to $\mathrm{UO}_{2.4}$ transition, the oxidation front progresses within each grain via diffusion of oxygen through an increasing thickness of the higher oxide. Thus, larger grains will take longer to oxidize than smaller grains. The grain size will also be important in determining what fraction of fuel may be suspended at various air flows. This grain size is a function of the initial grain size, any grain growth or subdivision that may occur during reactor operations, and the extent of intragranular cracking during oxidation. 


\subsubsection{Initial Grain Size and Growth}

Iwasaki et al. (1968) used unirradiated $\mathrm{UO}_{2}$ pellets in their oxidation studies. They report an average initial grain size of $9 \mu \mathrm{m}$. Similar studies on both unirradiated and irradiated $\mathrm{UO}_{2}$ pellets of CANDUTM fuel (Liu et al. 1992) also reported an average initial grain size of $9 \mu \mathrm{m}$. The HBU fuel recently studied by Spino et al. (2006) had initial grain sizes ranging from $9 \mu \mathrm{m}$ to $12 \mu \mathrm{m}$. Early versions of ASTM International standard C776 Standard Specification for Sintered Uranium Dioxide Pellets had the requirement that the average grain size of as-fabricated pellets should be $\geq 5 \mu \mathrm{m}$. More recent versions of this and similar standards state that "The performance of $\mathrm{UO}_{2}$ fuel pellets is affected by their grain size and pore morphology. These characteristics shall be mutually agreed upon between the buyer and the seller" (ASTM International C776-00).

Some of the performance factors influenced by grain size are FGR and the formation of the HBS. Une et al. (2000) have shown that large-grained fuels reduced FGR by about 30 to $50 \%$ relative to standard-grained fuels, even after achieving a rod average burnup of $75 \mathrm{GWd} / \mathrm{MTU}$. Similarly, the formation of the sub-divided or recrystalized grains associated with the HBS was greatly suppressed in large-grained fuels relative to the standard-grain fuels. It is highly probable that the grain size for newer fuels will be markedly larger than past fuels. These newer fuels will typically achieve much higher burnup, so it is likely that these innovations will limit the quantity of HBS available for dispersion.

Fuel vendors do not typically publish the characteristics of their fuel, and these may change not only from vendor to vendor, but for each of the cores they supply. However, examination of recent data suggests that initial grain sizes for modern fuels are larger than the $9 \mu \mathrm{m}$ reported by Iwasaki et al. (1968) and Liu et al. (1992). The fuel the Yucca Mountain Project identifies as ATM 109 is a BWR fuel irradiated in the Quad Cities 1 reactor. Vaidyanathan et al. (1997) characterized this fuel initially at GE Vallecitos Nuclear Center, and portions of the fuel rod were then sent to Argonne National Laboratory (ANL) and PNNL for examination and testing. Three different fuel types representing different ${ }^{235} \mathrm{U}$ enrichments and $\mathrm{Gd}_{2} \mathrm{O}_{3}$ content as a burnable poison were characterized. The two fuels without $\mathrm{Gd}$ are reported as having fuel grain sizes of $15 \mu \mathrm{m}$ and $30 \mu \mathrm{m}$. The Gd-doped fuel is reported only as having a bimodal grain size, with some grains in the $10-\mu \mathrm{m}$ to $12-\mu \mathrm{m}$ range and a second set interspersed with grains $<5 \mu \mathrm{m}$. PNNL purchased unirradiated $\mathrm{UO}_{2}$ pellets from a fuel vendor in 2000 for use in a testing program. The fuel came from 10 different lots from 6 different projects. The Gd content of the pellets ranged from 0 to $8 \mathrm{wt} \%$. The vendor reported grain sizes ranging from $12 \mu \mathrm{m}$ to $16 \mu \mathrm{m}$. It appears that larger grain sizes are indeed becoming the industry norm to help minimize FGR and HBS formation.

Knowing the grain size of the fuel will help determine the fraction of fuel that may be small enough to be airborne under various postulated accident scenarios. However, the initial grain size of as-fabricated fuel is not typically the same as the post-irradiation grain size. With the exception of HBS formation in the first few hundred microns, as discussed in Section 1.1.1, fuels experience grain growth during reactor operations. At the temperatures the fuel experiences during irradiation, grain growth occurs whereby the average grain size of the pellet increases. Thus, CSNF will have larger grains than reported by the vendor for unirradiated fuel. Grain growth typically occurs predominately towards the fuel centerline where temperatures are higher. Typically, the grains at the surface of the fuel experience little, if any, grain growth. 
Detailed grain-size distributions for the irradiated ATM fuels are available in their respective characterization reports (Guenther et al. 1988a, 1988b, 1991a, 1991b). It is clear from these data that grain growth depends on the temperature of the fuel and varies both radially and axially. Grain growth can be significant, as high as $116 \%$, for rods that experienced power excursions such as often result in larger-than-average FGRs. It is important to know the grain-size distribution of fuel before oxidation to truly assess the impacts on radionuclide release. Still, it can be readily argued that modeling as-fabricated grain sizes is conservative in that they are smaller than post-irradiation grain sizes, with the exception of the HBS.

\subsubsection{Post-Oxidation Particle Size}

As CSNF oxidizes, the first phase that forms is a $\mathrm{UO}_{2.4}$ or $\mathrm{U}_{4} \mathrm{O}_{9}$-like phase that is slightly denser than the starting $\mathrm{UO}_{2}$. This causes a slight contraction of the grain boundaries, allowing relatively free diffusion of oxygen. But this does not imply that the fuel falls apart into individual grains at this point. The fuel becomes friable, but may remain as large particles. Figure 2.3 shows a series of scanning electron microscope (SEM) images taken from a sample of ATM-105 (BWR fuel with a rod-average burnup of $28 \mathrm{GWd} / \mathrm{MTU})$ oxidized for 544 hours at $255^{\circ} \mathrm{C}$ to an oxygen-to-metal (O/M) ratio of 2.41 based on mass increase. Figure 2.3a shows the large particles that were sampled from the $\sim 200-\mathrm{mg}$ fragment that was oxidized. Some powder was created while the SEM mount was being prepared. Figure 2.3b and Figure 2.3c are an increased magnification of these larger particles showing that the grain boundaries are visible, but not separated. Figure $2.3 \mathrm{~d}$ shows the grain-sized particles formed during the sample preparation. From the scale, it is very clear that only a very minute fraction of the fuel is smaller than $10 \mu \mathrm{m}$. Thus, oxidation to only $\mathrm{UO}_{2.4}$ will provide only a very small amount of powder available for airborne release, unless a mechanical shock is applied to break the fuel apart at the grain boundaries.

As oxidation continues, $\mathrm{U}_{3} \mathrm{O}_{8}$, a phase much less dense than the starting $\mathrm{UO}_{2}$, is formed. Because it is less dense, it spalls from the fuel as it is formed. In addition to temperature affecting the oxidation kinetics, Iwasaki et al. (1968) demonstrated that the particle size tended to increase with oxidation temperature. At present, it is unclear if this finding applies to the lower temperature range expected for the pre-closure operations. Figure 2.4 shows the extent of intragranular cracking experienced by ATM-105 fuel (post irradiation grain size is $11 \mu \mathrm{m}$ to $15 \mu \mathrm{m}$ ) when subjected to complete oxidation to $\mathrm{U}_{3} \mathrm{O}_{8}$ at temperatures of $283^{\circ} \mathrm{C}, 305^{\circ} \mathrm{C}$, and $325^{\circ} \mathrm{C}$, in a, b, and c, respectively. In this case, it appears that the intragranular cracking, and thus the potential for creating particles smaller than a grain, is more extensive at higher temperatures. 

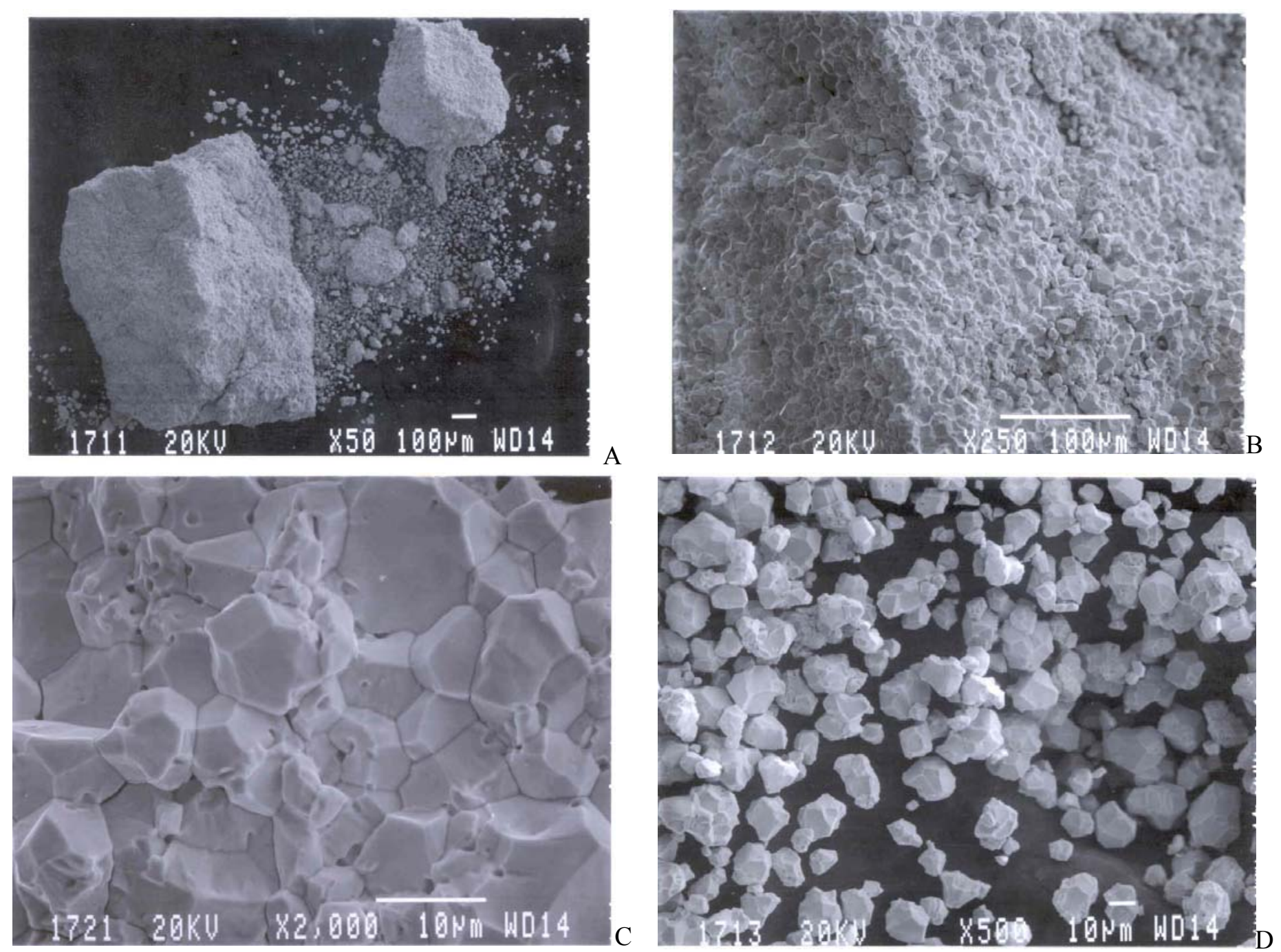

Figure 2.3. (a-d). Micrographs of Moderate Burnup BWR Fragments Oxidized to $\mathrm{UO}_{2.4}$ 

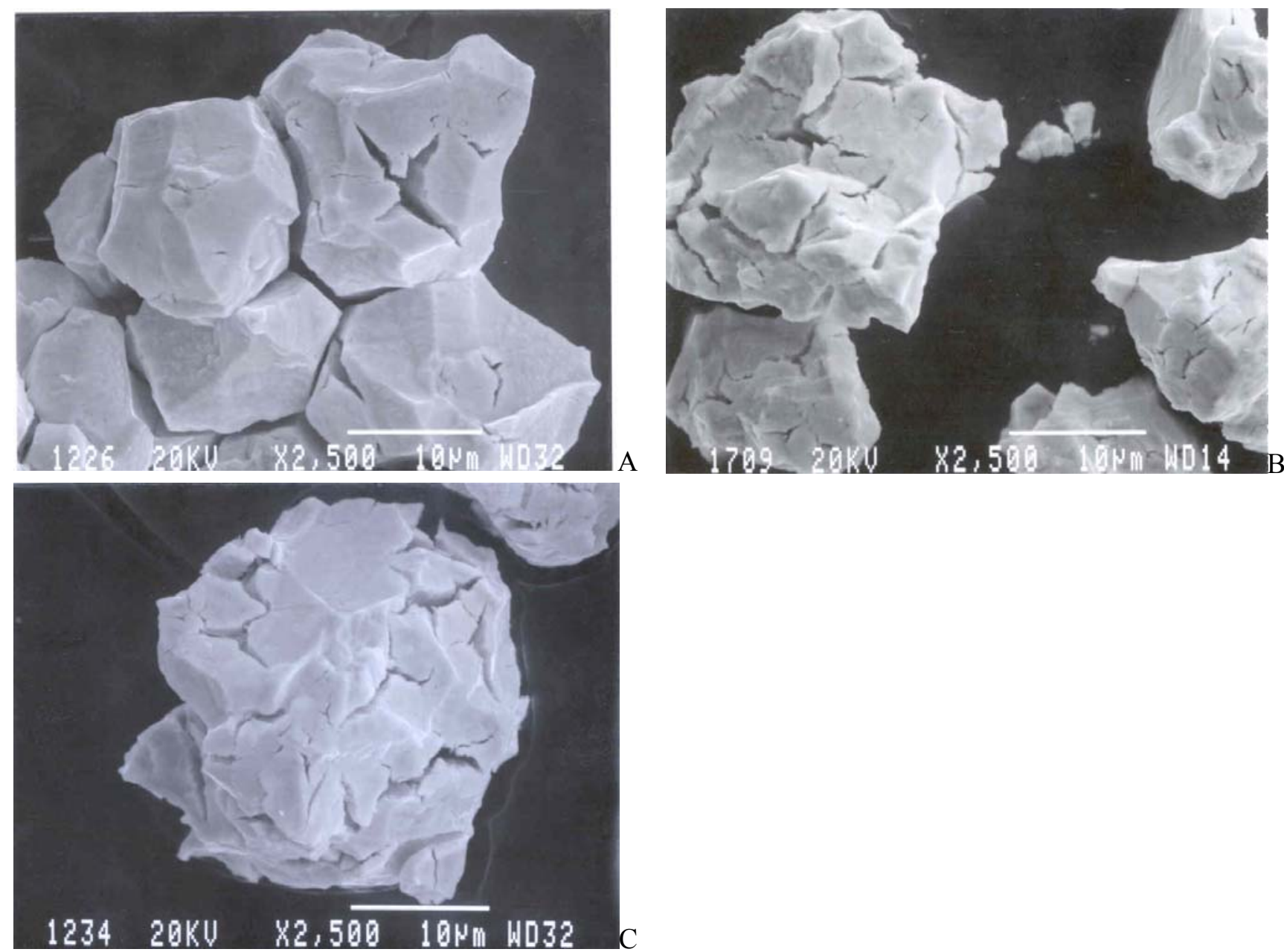

Figure 2.4. (a-c). Micrographs of Moderate Burnup BWR Fragments Oxidized to $\mathrm{U}_{3} \mathrm{O}_{8}$ at a) $283^{\circ} \mathrm{C}$, b) $305^{\circ} \mathrm{C}$, and c) $325^{\circ} \mathrm{C}$

\subsection{FY07 Oxidation Results}

Previous oxidation work in support of the YMP (e.g., Hanson 1998; CRWMS M\&O 2000) has shown the burnup dependence of CSNF oxidation at various temperatures relevant to post-closure scenarios. For this work, the higher temperatures relevant to off-normal conditions in the surface facilities were the focus. In addition, there was some question as to whether the highly porous and small-grained HBS would counteract the delays in burnup expected due to the chemical stabilization of higher burnup fuels. This work focused on the oxidation of fuel fragments known to have HBS present. A piece of fuel from ATM-109A (rod ZB00113 identified by ANL as 539F5) originally cut in December 1998 for other oxidation experiments was retrieved from the PNNL hot-cell archive. This fuel is identified by Vaidyanathan et al. (1997) as having an initial ${ }^{235} \mathrm{U}$ enrichment of $3.80 \%$, and a burnup, based on ${ }^{137} \mathrm{Cs}$ counting, in the range $59.2 \mathrm{GWd} / \mathrm{MTU}$ to $66 \mathrm{GWd} / \mathrm{MTU}$. The archived sample was a thin piece of fuel still attached to the cladding (see Figure 2.5). The upper piece of fuel was separated from the cladding by laying the entire specimen on a paper towel in the hot cell and then tapping it with a "hammer." The fuel 
separated nicely from the cladding. It is possible that this piece of fuel includes some of the $\mathrm{Zr}$ liner (ATM-109 was a barrier fuel with a liner of pure $\mathrm{Zr}$ on the fuel side of the cladding). The upper piece of fuel, which was broken in two, clearly has the HBS material and minimizes the amount of non-rim material. These two fragments were then oxidized at $450^{\circ} \mathrm{C}$ and $500^{\circ} \mathrm{C}$, respectively, as described below. A similar process of separating fuel from the cladding was repeated to obtain the second set of fragments for the oxidation experiments performed at $350^{\circ} \mathrm{C}$ and $400^{\circ} \mathrm{C}$.

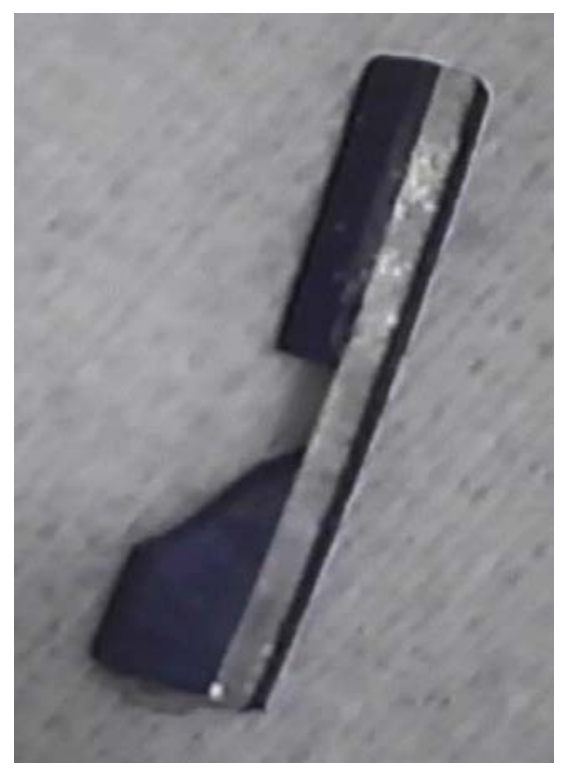

Figure 2.5. Fuel from ATM-109 Used for Oxidation Testing at $450^{\circ} \mathrm{C}$ and $500^{\circ} \mathrm{C}$. The upper piece was removed from the cladding and broken in two for testing.

Two systems were used to perform the oxidation experiments. Both systems (see Figure 2.6) consisted of an overhead hanger from which the fuel sample was suspended, a furnace and a proportionalintegral-differential (PID) feedback controller to regulate the sample temperature, a closed gas-circulating system, and a computerized data-acquisition system. The sample temperatures were measured with a calibrated Chromel-Alumel thermocouple positioned immediately below a quartz crucible that served as the sample container.

A single fuel fragment was placed in the quartz crucible and then weighed to obtain the initial mass. Since the fuel tested this fiscal year was from a HBU rod, the mass of each individual fragment was limited to less than $150 \mathrm{mg}$ due to dose constraints. The crucible was then suspended on a platinum chain from the overhead hanger. A quartz reaction tube was then raised to enclose the sample. The system was evacuated to a pressure less than 50 millitorr for at least 12 hours. Commercial dry air (Scott's Specialty Gas) containing $21 \%$ oxygen with less than 3 ppm $\mathrm{H}_{2} \mathrm{O}$ was then admitted to the system at a pressure of between 700 torr and 747 torr. A gas circulation pump directed air flow downward onto the sample at a total flow rate of $200 \mathrm{cc} / \mathrm{min}$. Once the furnace was turned on, the fuel sample reached the desired operating temperature (maximum of $500^{\circ} \mathrm{C}$ ) within 2 hours, at which time the operating pressure was approximately 760 torr. The sample temperature, system pressure, and gas recirculation rate were monitored and recorded every minute on a LabView ${ }^{\mathrm{TM}}$ data-acquisition system. The oxidation was allowed to proceed, with minor adjustments made to keep the sample at the desired temperature, until the 
time at temperature was approximately 30 days, the time estimated that fuel may be exposed to oxidizing conditions during off-normal conditions involving fuel in the surface facilities at Yucca Mountain. Upon unloading, the fragment was reweighed to determine the mass increase due to oxidation. This mass increase has relatively large uncertainties since the sample is weighed inside a capped vial (see Figure 2.7) to prevent the spread of radioactive particulates. The uncertainty results from a total mass change of only a few $\mathrm{mg}$ in a system that weighs approximately $20 \mathrm{~g}$. The change in the $\mathrm{O} / \mathrm{M}$ ratio can be calculated from the mass increase of the specimen using the equation

$$
\Delta(\mathrm{O} / \mathrm{M})=(270 / 16) \times\left(\Delta \mathrm{M} / \mathrm{M}_{0}\right)
$$

where $270=$ atomic mass of $\mathrm{UO}_{2}$ (the mass difference due to fission of $\mathrm{U}$ and substitution of fission products and higher actinides is ignored)

$16=$ the atomic mass of the oxygen taken up by the sample (i.e., assumes that the only mechanism for mass increase is oxygen uptake)

$\Delta \mathrm{M}=$ increase in mass

$\mathrm{M}_{0}=$ original mass of the specimen.

Tests were run at $350^{\circ} \mathrm{C}, 400^{\circ} \mathrm{C}, 450^{\circ} \mathrm{C}$, and $500^{\circ} \mathrm{C}$. Examination of the samples after oxidizing for at least 30 days at the two lower temperatures showed that the samples remained as fragments. Even when force was applied with tweezers, the fragments that oxidized at $350^{\circ} \mathrm{C}$ and $400^{\circ} \mathrm{C}$ did not break apart. This suggests that no $\mathrm{U}_{3} \mathrm{O}_{8}$ was formed. This is substantiated by mass increases corresponding to increases in $\mathrm{O} / \mathrm{M}$ to only about 2.20 to 2.23 . While these $\mathrm{O} / \mathrm{M}$ ratios are quite low relative to the normal plateau of approximately 2.40 , it is necessary to stress that the plateau has not been previously measured on fuels with burnups as high as these fuels.

Each of the fragments oxidized at $350^{\circ} \mathrm{C}$ and $400^{\circ} \mathrm{C}$ was counted for 1 hour using a track detector at a distance of about 2 meters from the face of the detector. The gamma self-absorption in each sample was determined empirically by adjusting the average sample thickness to give the best fit for multiple gamma emission lines from ${ }^{134} \mathrm{Cs}$, ${ }^{154} \mathrm{Eu}$, and ${ }^{155} \mathrm{Eu}$. When normalized to the pre-oxidation sample mass, both samples had a normalized ${ }^{137} \mathrm{Cs}$ concentration of $0.118 \mathrm{Ci}$ per $\mathrm{g} \mathrm{UO}_{2}$, or $0.134 \mathrm{Ci}$ per g U. The SCALE code was run for a fuel with a 3.8\% enrichment and 15-year decay for burnups of $55 \mathrm{GWd} / \mathrm{MTU}$ and $60 \mathrm{GWd} / \mathrm{MTU}$. Using linear interpolation to determine the change in ${ }^{137} \mathrm{Cs}$ concentration with burnup, the average burnup of the two fragments was estimated as $65 \mathrm{GWd} / \mathrm{MTU}$. 


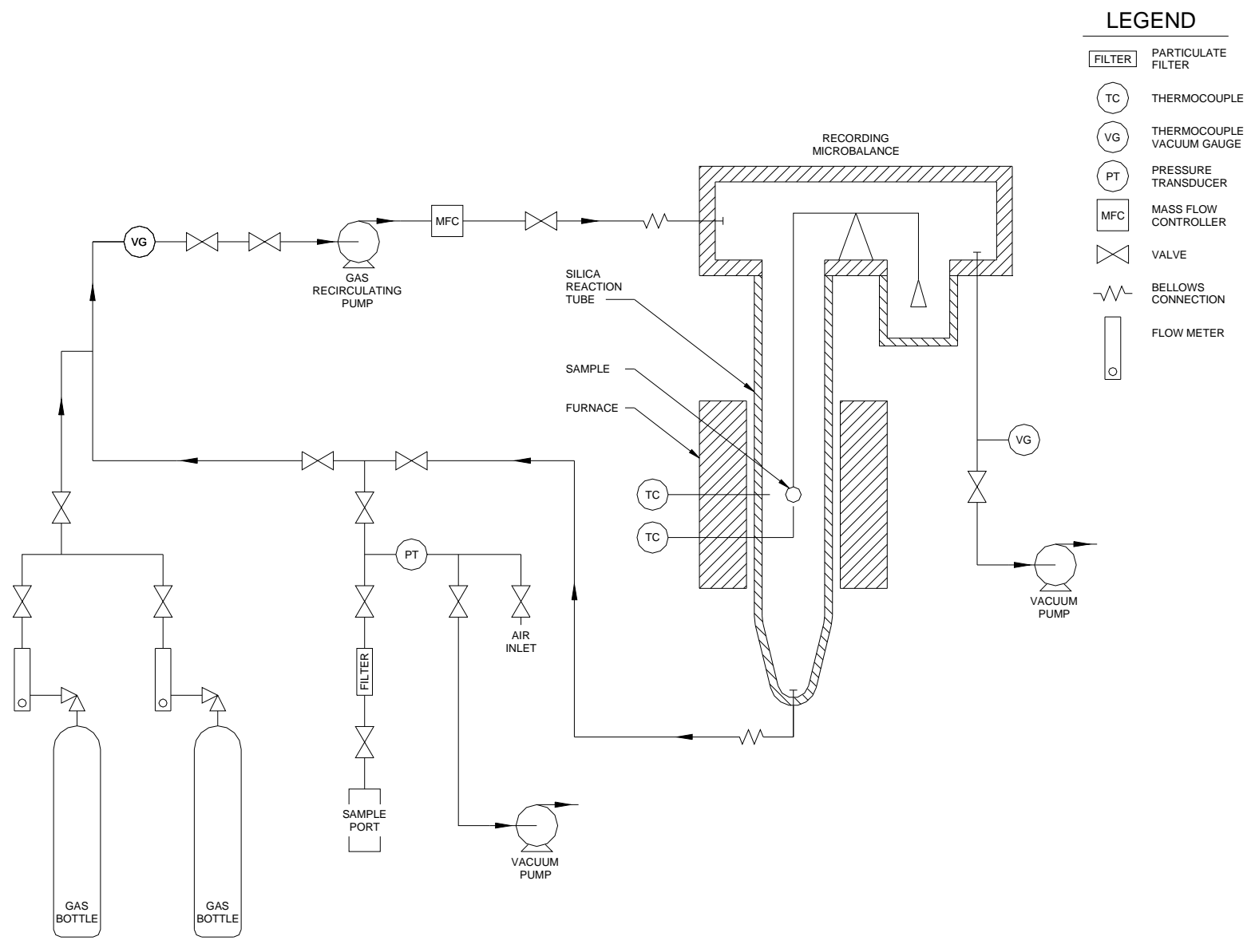

Figure 2.6. Schematic of Oxidation Apparatus

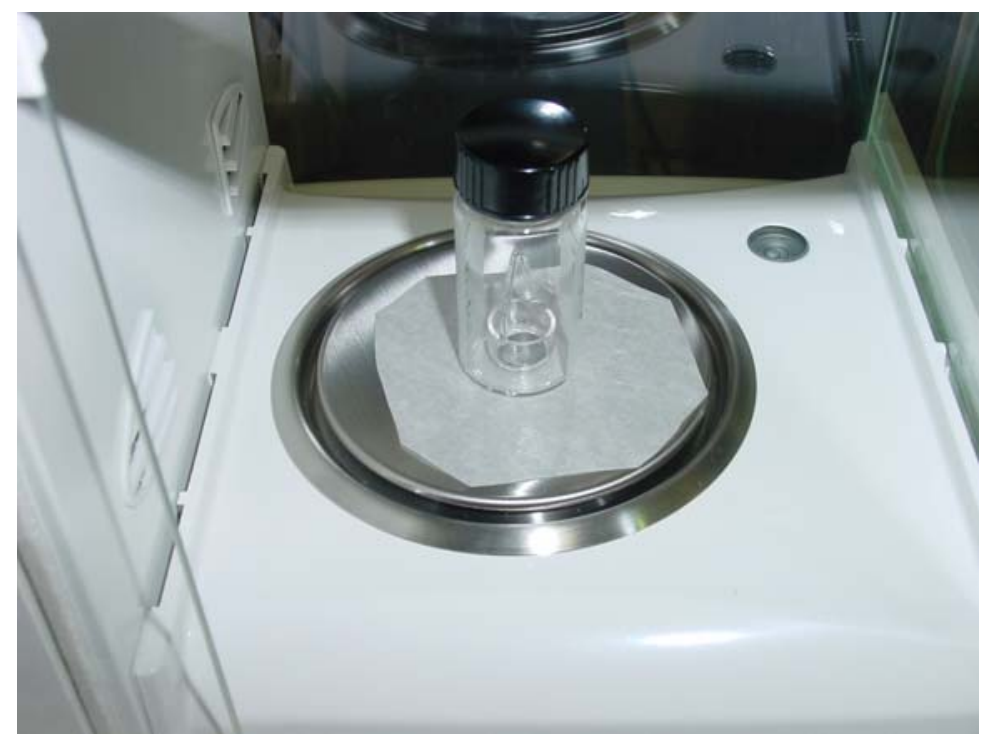

Figure 2.7. Weighing of Sample After Oxidation 
Using the methodology for calculating the incubation time for clad unzipping due to fuel oxidation found in CRWMS M\&O (2000) as expressed in Equation 2.2, the time for incubation for fuel with a burnup of $65 \mathrm{GWd} / \mathrm{MTU}$ at $400^{\circ} \mathrm{C}$ is approximately 17 hours. This fuel oxidized for over 720 hours without signs of $\mathrm{U}_{3} \mathrm{O}_{8}$ formation, although $\mathrm{X}$-ray diffractometry has yet to be performed. Still, this suggests that the burnup dependence of oxidation is not linear as presented in CRWMS M\&O 2000, but fuel is more resistant to oxidation at higher burnups than currently modeled. To verify that diffusion of $\mathrm{Zr}$ from the liner of the cladding did not influence the oxidation results, these tests will be repeated with fuel from the center of the pellet that will not have the HBS and will have a slightly lower average burnup. Another conclusion drawn from these tests is that the presence of the HBS did not accelerate oxidation because of the fine-grained, high-surface-area structure.

The two samples oxidized for at least 30 days at $450^{\circ} \mathrm{C}$ and $500^{\circ} \mathrm{C}$; however, were completely disintegrated into powder (see, e.g., Figure 2.8) even before being unloaded from the system. The mass increase for both samples yields final $\mathrm{O} / \mathrm{M}$ ratios of $\sim 3.0$, suggesting that $\mathrm{UO}_{3}$ was formed. While the formation of $\mathrm{UO}_{3}$ is not normally observed without the presence of water vapor during the oxidation, data at these higher temperatures are not as readily available, and we must assume that this phenomenon is real. SEM was performed on subsamples of each of the powders to try and determine particle sizes. As seen in Figure 2.9 and Figure 2.10, there is a large range of particle sizes present with what appears to be a large fraction of particles smaller than $2 \mu \mathrm{m}$. It is interesting to note that intragranular cracking is not noticeable in either Figure 2.9 or Figure 2.10, suggesting that all fragmentation has already occurred. However, it is clear from examining the micrographs that using SEM was not going to yield accurate particle-size distributions (PSDs), which led to the development of the tests discussed later in this report. 


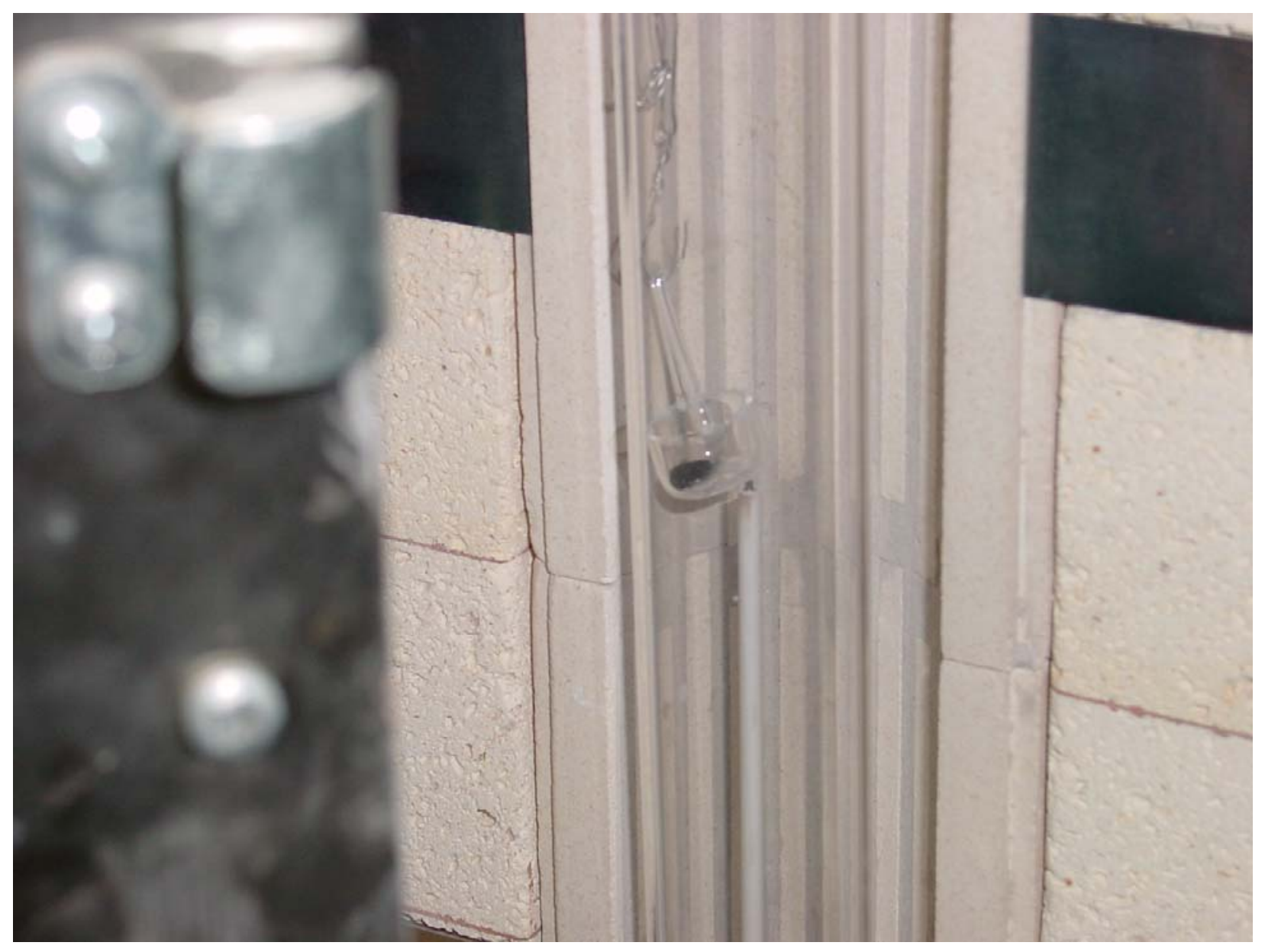

Figure 2.8. ATM-109 Fuel Fragment Oxidized for Approximately 30 Days at $500^{\circ} \mathrm{C}$ Disintegrates into Powder 

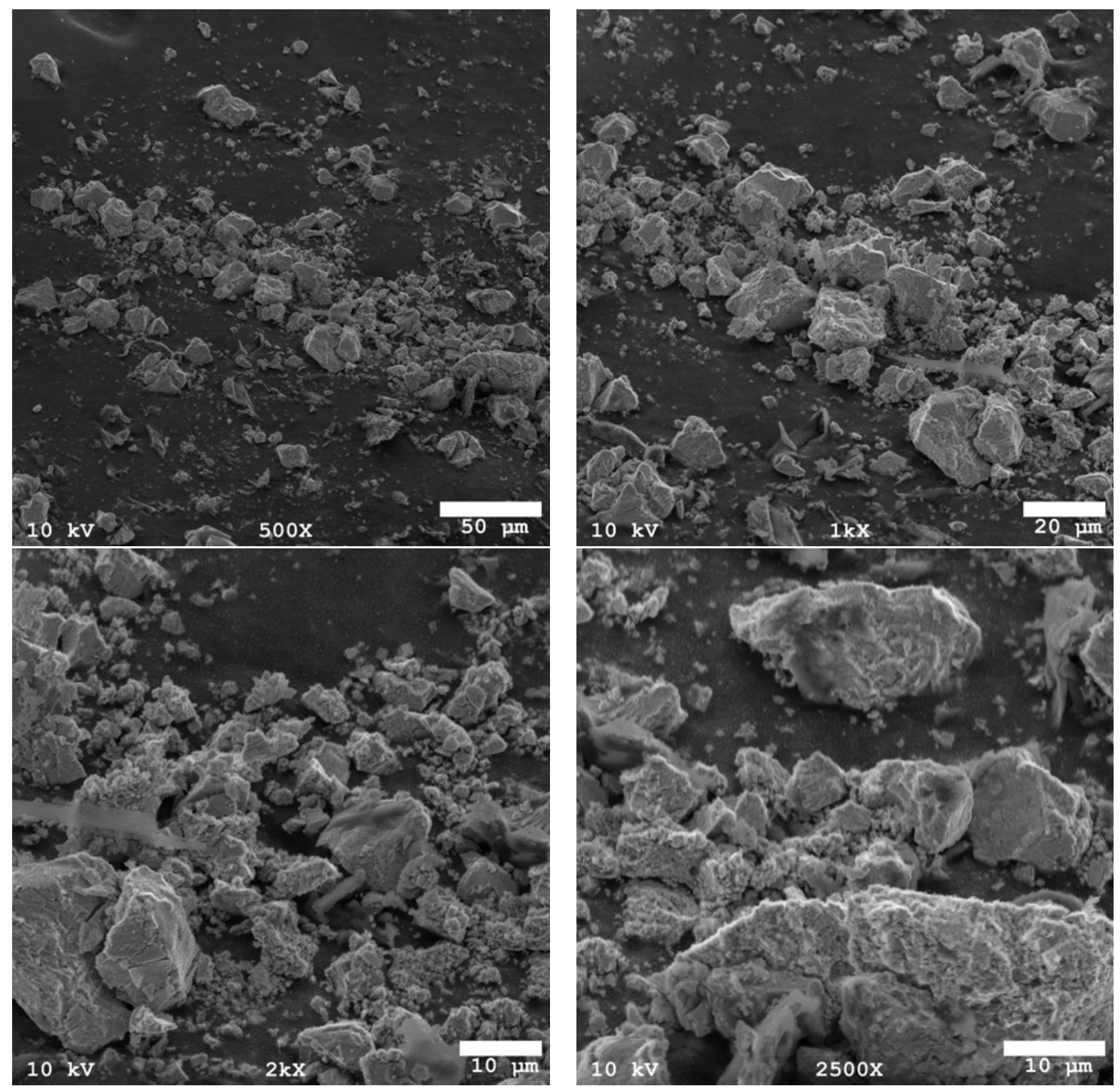

Figure 2.9. SEM Images of ATM-109 Fuel Oxidized at $450^{\circ} \mathrm{C}$ for Approximately 30 Days 

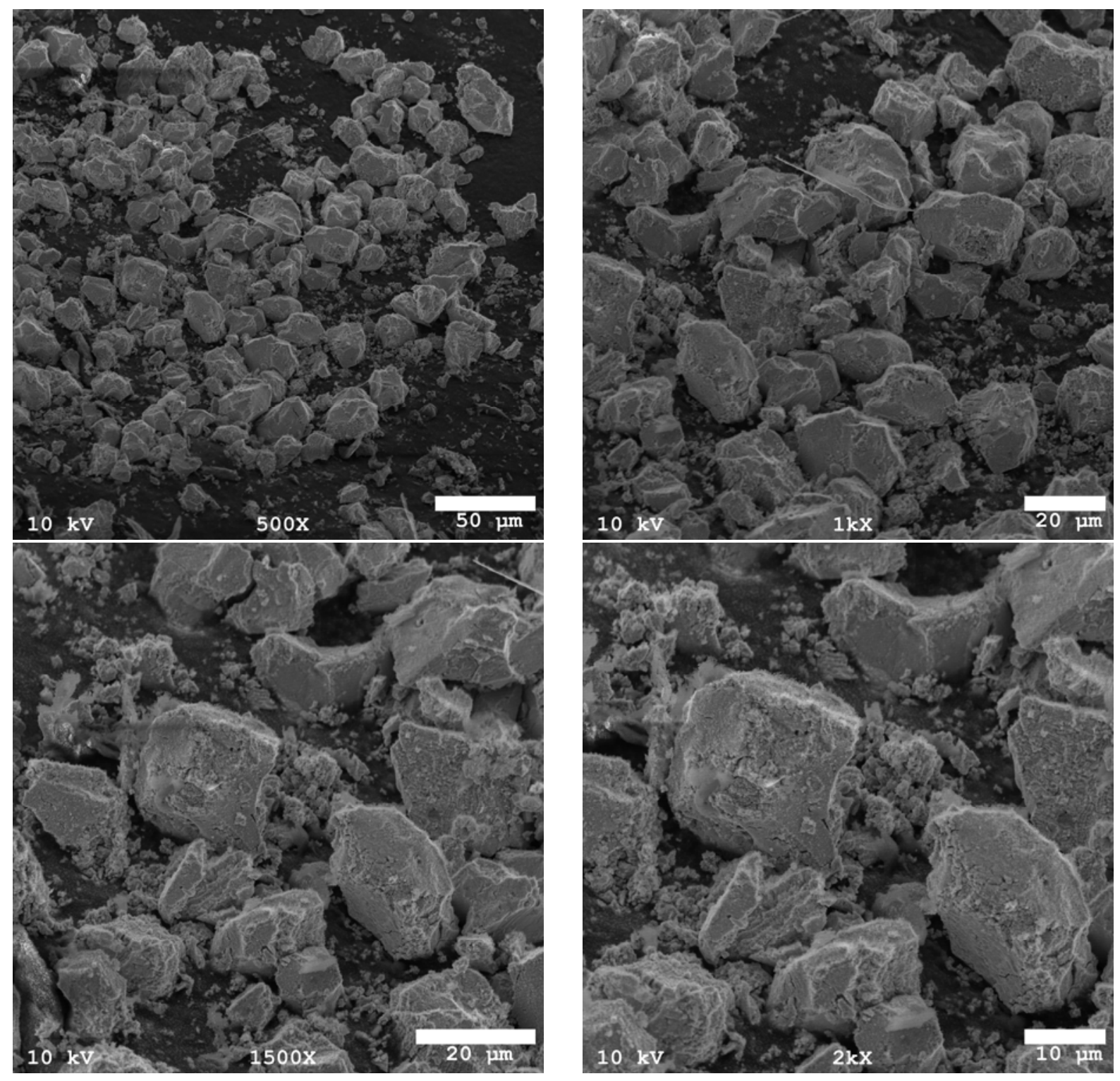

Figure 2.10. SEM Images of ATM-109 Fuel Oxidized at $500^{\circ} \mathrm{C}$ for Approximately 30 Days 


\subsection{CSNF Tests}

The goals of experimental testing with CSNF rods and powders are to provide a conservative (maximal) estimate of total and airborne spent-fuel release fractions during canister breach or failure events, a defensible range of particle sizes released, and data to determine the effect of HBU on release. Failure scenarios emphasize the convective entrainment and transport of nuclear fuel in air. Experimental work was formulated based on a "worst-case" scenario, where the CSNF material being tested was placed in direct contact with the suspending medium in such a way as to maximize potential release. Two subcategories of release were examined: 1) unoxidized nuclear fuel released from a "breached" rod and 2) dispersion of oxidized powder released from a cylindrical sample boat (discussed in more detail in the following pages).

\subsection{Breach Test on Unoxidized Fuel}

Test subcategory \#1 simulates the release of unoxidized HBU fuel from a rod caused by a breach of the cladding. Because fuel rods have significant internal pressure, any accidental rupture of the cladding would be accompanied by a release of pressure and an outflow of gas from the rod through the breach. The geometry of the cladding breach will determine the rate and duration of pressure equilibration. For example, a small breach could provide a sustained, low volumetric flow rate from the rod. On the other hand, a large breach could provide a sudden, relatively high volumetric flow rate from the rod. In addition, the geometry of the breach also can influence the amount of material released (e.g., 30- $\mu \mathrm{m}$ fuel fragments could not escape through a $10-\mu \mathrm{m}$ crack in the cladding).

With this in mind, a diverse range of breach scenarios could be postulated. However, for the purpose of simulating a "worst-case" breach event, the release of unoxidized nuclear fuel from "breached" rods was accomplished by flowing (forcing) a continuous stream of dry air through the porous internal structure of cladded CSNF segments. Specifically, a 1- to 2-in. segment of an HBU CSNF rod was cut so that unoxidized fuel was exposed at both ends. One end was attached to a dry air cylinder using a Swagelok fitting. The open end was connected to a cylindrical chamber being monitored for fuel/particle release. Depending on the configuration of the fuel within the cladding, the air may flow through the annular gap between the rod and cladding (if the fuel has not expanded to close this gap as is typical for HBU fuels) or through void space within the rod itself. During irradiation, the fuel pellets crack and fragment because of the large temperature gradients over very small distances. This fragmentation, as shown in Figure 3.1 for a segment of fuel from a companion rod from ATM-106 (Guenther et al. 1988b, Figure E.1.g), creates void space, even if the fuel-clad gap is closed..

Because the crack and void geometries inside the fuel rod are of such a small scale, local air flow velocities can be high, allowing the air to shear or erode particles off the surface of the fuel or dislodge entire sections of fuel. These entrained particles can redeposit on fuel surfaces downstream of the source region or be carried out of the rod by the air stream. The latter comprises a fuel release from the rod. During breach testing, the fuel rod is subjected to a range of gas flows from zero up to a predefined maximum and to a variety of agitation conditions such as vibration (by tapping the fuel rod) or pressure pulses (by rapid increase in supply pressure). 


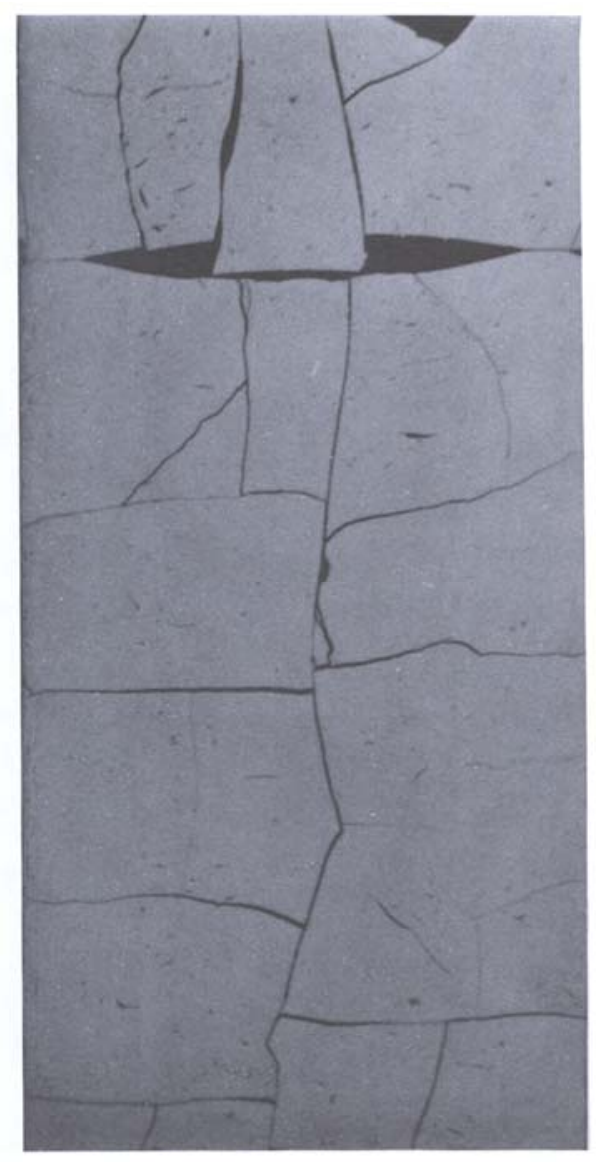

Figure 3.1. Example of Pellet Cracking and Flow Path for ATM-106 rod NBD-107 (taken from Guenther et al. 1988b, Figure E.1.g)

The setup described in the preceding paragraph constitutes a "worst-case" breach in two respects. First, the breached area is significant, with the entire axial cross-section exposed to the atmosphere and nothing to hinder particulate release from the open end. A more realistic breach in the cladding would be a crack on the micro- to millimeter length scale. Second, the total mass of air that flowed through the fuel rod during the course of breach testing is substantially higher than the mass of pressurized gas contained in an intact rod. Indeed, during breach tests, the fuel rods were exposed to gas flow rates of 0 to 9000 standard cubic centimeters per minute (SCCM) over periods ranging from 1 to 3 hours. This prolonged contact with gas flow provides a greater opportunity for fuel erosion and release than could be anticipated under postulated accident scenarios. Similarly, each rod segment was tested multiple times, increasing the potential for dislodging and releasing particles because of the large volumes of gas that flowed through the sample. A detailed discussion of the potential flow rates a breached rod may experience is found in Section 4.3.

\subsection{Suspension Test on Oxidized Fuel Powders}

Test subcategory \#2 is aimed at determining the "true" PSD of fuel samples that have been oxidized, as described in Section 2.3. In terms of postulated accident scenarios, it simulates the interaction of oxidized fuel fragments with an air stream. Unlike the fuel elements in the breach tests, oxidized fuel 
forms a fine powder. Because the fuel is already fragmented, it is dispersed simply by placing it in a container through which air is flowed. Here, the entire powder will be engulfed by the air stream, which is thought to provide an upper bound of the possible release fraction.

As with the tests on fuel rod segments, an inline optical particle counter is used to monitor and analyze any entrained particles in the gas stream leaving the sample container. The flow rate of gas is varied stepwise from near zero up to a predefined maximum. Each flow set point will be maintained until the concentration of particles detected falls below a predefined limit. As with the breach tests, vibration and pressure pulses are employed to assist or facilitate dispersion.

\subsection{Test Apparatus Description}

Release of particulates from unoxidized fuel segments and oxidized fuel powders were measured with PNNL's Fuel-In-Air Test Apparatus (FIATA). The FIATA was designed to create and analyze dispersions of CSNF particles by 1) fragmenting unoxidized fuel pellets in cladded CSNF rods or 2) fluidizing oxidized CSNF powder. It was designed for the characterization of both radioactive and non-radioactive materials. The FIATA is a composite system composed of both commercial off-the-self laboratory equipment and fabricated hardware. It consists of the following subsystems:

- gas dispersion

- dispersion confinement enclosure

- dispersion collection/analysis

The gas-dispersion system flows air through the material being tested, allowing fluidization and dispersion of any loose particulate material. It consists of a compressed dry air gas cylinder, two flow controllers spanning flows from 1 to 10,000 SCCM, and the sample holder for the fuel segment or powder. Two separate sample adaptors have been designed: 1) one that allows a fuel-rod segment to be tightly fitted to the air flow line using a Swagelok ${ }^{\circledR}$ fitting such that air flows through the porous interior of the fuel rod and 2) another that allows gas flow through a loose powder resting on top of a porous metal plug through which air is flowed. Both setups are similar, with the primary difference being that powder tests employ a 0.5 -in. OD cylindrical sample boat with a porous metal base in place of a fuel-rod segment. Figure 3.2A shows a basic schematic of the sample holder.

The dispersion confinement enclosure is a cylindrical housing (approximately 2 inches in diameter and 7 inches in length) that prevents particulate matter dispersed by the gas-dispersion system from spreading beyond the FIATA. As shown in Figure 3.2B, the enclosure fits over the sample holder and limits particle dispersion to the annular region between the boat and the enclosure wall. The enclosure serves as a localized space from which the dispersed particles can be collected, sampled, and characterized. The air balance is maintained by drawing air into (or pushing air out of at higher flow rates) multiple filtered ports. This air balance is necessary because the cascade impactors require a constant $9 \mathrm{~L} / \mathrm{min}$ input while the optical particle counters have a maximum 1-L/min input.

The dispersion collection/analysis system consists of a collection filter (connected to a downstream vacuum pump) or inline particle analyzer. The specific system used depends on whether the particles are being analyzed in real-time or being collected for post-experimental analysis. For real-time analysis, 
inline optical particle counters are used. If particles are being collected for later analysis, an inline filter or cascade impactor connected to a downstream vacuum pump will be used.

Two FIATAs were set up at PNNL. A non-radiological system was setup in the general laboratory space in the Radiochemical Processing Laboratory (RPL). A separate radiological FIATA was set up in the RPL's Shielded Analytical Laboratory (SAL). All work on CSNF rod segments and powders was performed using the SAL FIATA.

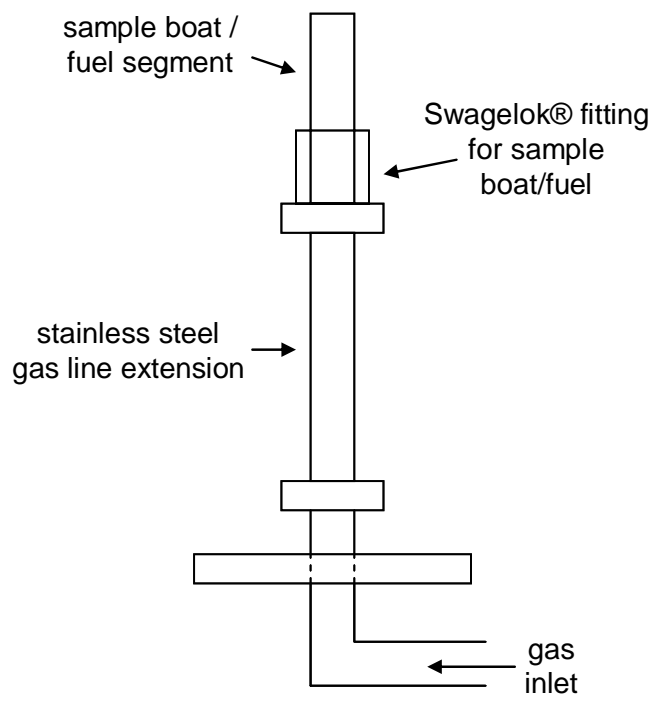

(A)

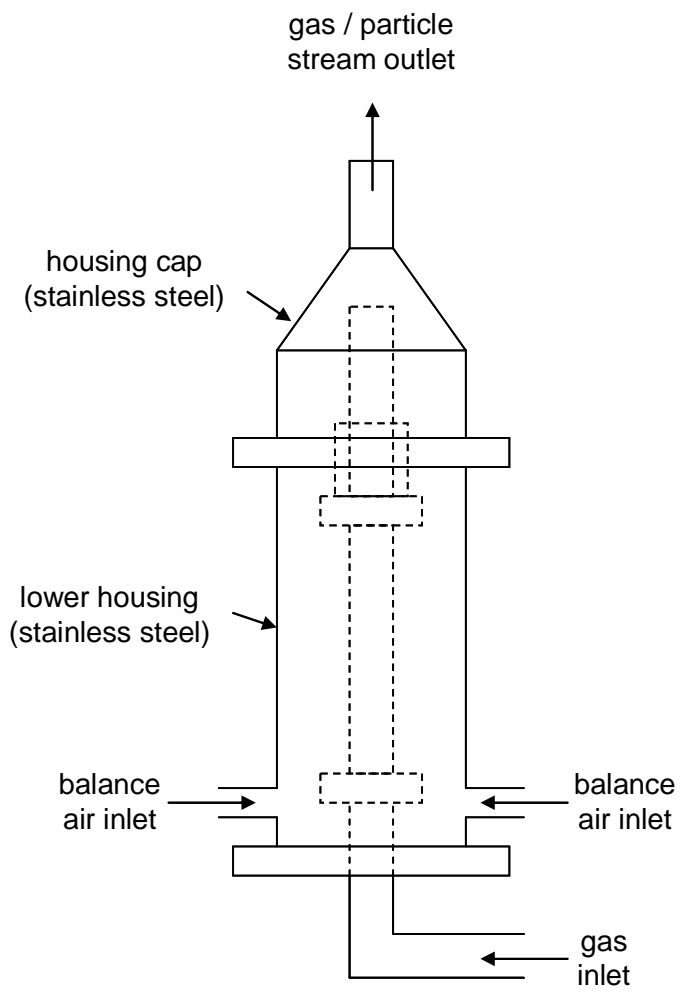

(B)

Figure 3.2. The Sample Holder (A) Consists of a Gas Line Inlet Connected to a Compressed Dry Air Gas Cylinder and a Swagelok ${ }^{\circledR}$ Fitting to Secure the Fuel Segment or Sample Boat Being Tested. During testing, fuel particles or fragments are confined within a housing that directs air flow to the downstream detectors (B).

The SAL FIATA (shown in Figure 3.3) consists of a gas supply cylinder (compressed dry air) connected to two calibrated flow controllers, FC1 and FC2. FC1 is intended for low-flow applications ( 0 to 200 SCCM), whereas FC2 is intended for higher flow applications ( 0 to 10,000 SCCM). A pressure gage is located immediately downstream of the gas supply and is used to monitor the supply pressure. A pressure relief valve (set at $150 \mathrm{psig}$ ) is used to protect the flow controllers from over pressure. Valves V1, V2, and V3 are used as on/off switches for gas flow into the hot cell where the fuel rods and oxidized powders are located. A check valve is located just before the cell wall to prevent the back flow of radiological material out of the hot cell. 


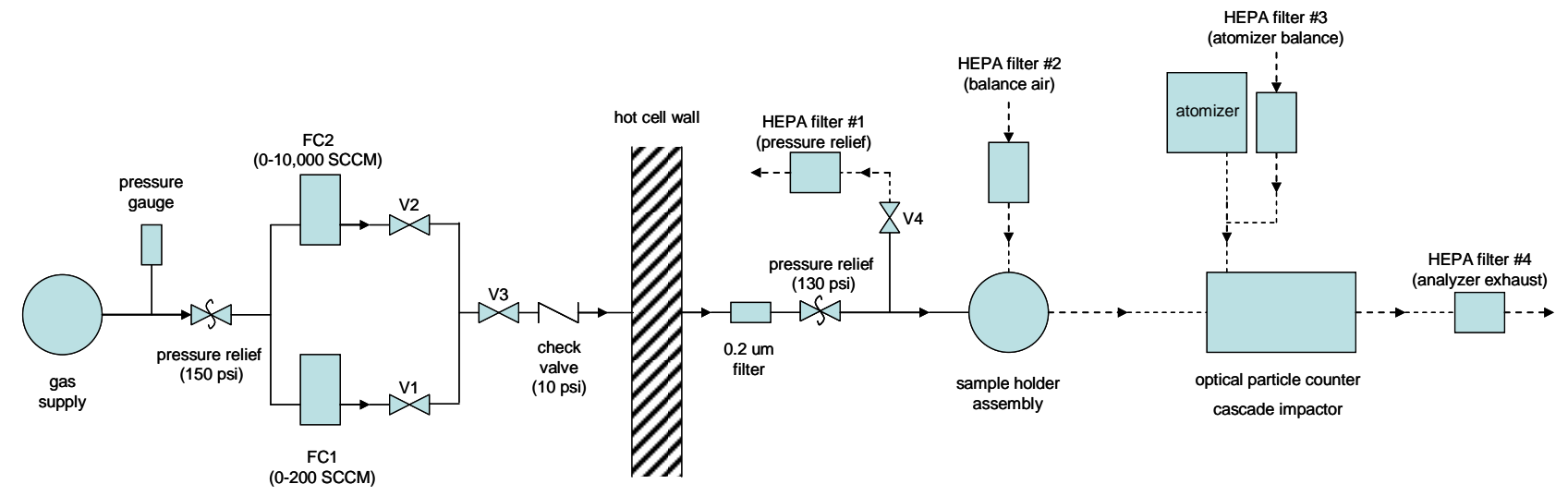

Figure 3.3. Fuel-in-Air Test Apparatus Setup Used for Testing CSNF Rod Segments and Powders at the RPL's SAL. Solid lines indicate stainless steel lines; dashed lines indicate plastic or conductive silicon tubing.

Here, gas flows from the supply bottle on the left of the diagram through the flow controllers (which throttle flow to the desired level) and into the hot cell. Once inside the hot cell, the dry air is first passed through a $0.2-\mu \mathrm{m}$ filter to remove any entrained particulates. The filter effluent then passes through another pressure-relief valve (set at $130 \mathrm{psig}$ ) and into the sample holder assembly shown in Figure 3.2. When passing through the sample chamber, the dry air interacts with the sample (either oxidized fuel powder or a cladded fuel segment) and potentially picks up fuel fragments/particles. Air then flows into the sample chamber enclosure where it mixes with balance air filtered through high-efficiency particulate air (HEPA) filter \#2, where it is sampled by downstream optical particle counters or cascade impactors. During analysis, any entrained fuel particles are cleaned from the air stream using inline HEPA filters (HEPA \#4). The cleaned air is then exhausted to the inside of the hot cell. After each experiment, any residual pressure in the SAL FIATA is relieved through valve V4. Gas-flow tubing inside the hot-cell environment is either stainless steel, plastic tubing, or conductive silicon tubing. In Figure 3.3, stainless steel tubing is shown as solid connective lines whereas plastic tubing and conductive tubing are shown as dashed lines.

The optical particle counters used to analyze the dispersed fuel fragments are calibrated instruments whose performance needs to be validated periodically. For this purpose, an atomizer is included in the SAL FIATA setup. During optical-counter validation, the atomizer is used to disperse aqueous suspensions of highly uniform monodisperse polystyrene latex (PSL) spheres whose size is certified by the National Institute of Science and Technology (NIST). The size distribution of particles in the PSL aerosol is determined using the optical particle counter and compared to the size listed on the certificate to verify whether the counter is still functioning properly. Verification is performed before and after each series of measurements (defined as the period over which the OPC remains powered on, but typically 1 to 2 days).

\subsection{Test Apparatus Equipment}

As stated before, the SAL FIATA is composed of both off-the-shelf commercial instruments and fabricated hardware. Table 3.1 details the important equipment source and operation parameters. Detailed discussion is given in the following paragraphs. 
Table 3.1. Summary of Major Equipment Used for SAL FIATA

\begin{tabular}{|c|c|}
\hline Part & Description \\
\hline Gas Supply Cylinder & $\begin{array}{l}\text { Contains industrial-grade compressed air obtained from } \\
\text { American Air Liquide (Houston, TX 77056). }\end{array}$ \\
\hline Pressure Gauge & $\begin{array}{l}\text { A Baratron }{ }^{\circledR} \text { General Purpose Absolute Capacitance } \\
\text { Manometer Type 722A from MKS Instruments } \\
\text { (Wilmington, MA 01887). }\end{array}$ \\
\hline $\begin{array}{l}\text { Mass Flow Controllers FC1 and } \\
\text { FC2 }\end{array}$ & $\begin{array}{l}\text { Gas-flow controllers FC1 and FC2 are 1179A Mass-Flo }{ }^{\circledR} \\
\text { General Purpose Mass Flow Controllers from MKS } \\
\text { Instruments (Wilmington, MA 01887). FC1 is rated for } \\
200 \text { SCCM Air; FC2 is rated for 10,000 SCCM Air. }\end{array}$ \\
\hline Sample Holder Assembly & $\begin{array}{l}\text { Fabricated by PNNL. The gas line for the sample } \\
\text { boat/fuel segment is } 0.5 \text {-in. OD and } 0.38 \text {-in. ID. The } \\
\text { housing diameter is approximately } 2 \text { inches. The housing } \\
\text { height is } 7 \text { inches. The upper housing tapers down to a } \\
3 / 8 \text {-in. barb. The air balance inlet ports are } 1 / 4 \text { inch. }\end{array}$ \\
\hline HEPA Filters & $\begin{array}{l}\text { Whatman HEPA-Cap Disposable Air Filtration Capsule } \\
\text { No. } 75 \text { from Fisher Scientific (Waltham, MA 02454) }\end{array}$ \\
\hline Cascade Impactors & $\begin{array}{l}\text { A Sioutas Cascade Impactor with Leland Legacy }{ }^{\circledR} \text { Pump } \\
\text { Kit. Obtained from SKC West Inc. (Fullerton, CA 92834- } \\
\text { 4133). }\end{array}$ \\
\hline Optical Particle Counters & $\begin{array}{l}\text { A 16-channel profiler ( } 0.5 \text { to } 20 \mu \mathrm{m}) \text { model } 61636 \text { from } \\
\text { Met One Instruments, Inc (Grants Pass, OR 97526) }\end{array}$ \\
\hline Conductive Silicon Tubing & $\begin{array}{l}\text { Conductive silicone tubing for particle transport, 0.19-in. } \\
\text { inside diameter (fits } 1 / 4 \text {-in. barb), from TSI Incorporated } \\
\text { (Shoreview, MN 55126). }\end{array}$ \\
\hline Atomizer & $\begin{array}{l}\text { A Model } 3079 \text { Portable Atomizer Aerosol Generator from } \\
\text { TSI Incorporated (Shoreview, MN 55126). }\end{array}$ \\
\hline
\end{tabular}

The gas-supply cylinder employed contained industrial-grade compressed air and was obtained from American Air Liquide (Houston, TX 77056). All pressure gages and flow controllers were obtained from MKS Instruments (Wilmington, MA 01887). Flow controllers FC1 and FC2 are 1179A Mass-Flo® General Purpose Mass Flow Controllers. FC1, used to provide the lower range of gas flow rates tested, was rated from 4 to 200 SCCM Air. Control flow rates below 4 SCCM were possible, but not reliable. FC2, used to provide the higher range of gas flow rates tested, was rated from 200 to 10,000 SCCM Air. The flow controllers were rated up to working pressures of 150 psig. As such, an MKS Baratron ${ }^{\circledR}$ General Purpose Absolute Capacitance Manometer Type 722A was used to monitor the pressure upstream of the flow controllers for overpressure.

The sample holder assembly shown in Figure 3.2 was fabricated at PNNL. It consists of a 0.5-in. OD gas supply line that has been welded to a 3-in.-diameter flange plate, which forms the bottom of the enclosure. The gas supply line extends about 5.75 inches above the enclosure base and is capped by a Swagelok ${ }^{\circledR}$ fitting with a Teflon ${ }^{\circledR}$ ferule. The fitting accepts 0.5 -in.-OD sample boats or fuel rod 
segments (which have outer diameters slightly less than 0.5 inches). When tightened down with a fuel rod segment or powder sample boat in place, the Swagelok ${ }^{\circledR}$ forms a tight seal around the outer diameter of the sample. As a result, all air flows through the sample rather than around it. Teflon ${ }^{\circledR}$ was selected as the ferule material because it allowed greater variability in the diameter of the sample boat/fuel rod segment while still providing a tight seal.

Sample boats for powder samples were also fabricated at PNNL. They consisted of a 1.5-in.-long stainless steel tube ( 0.5 in. OD, 0.37 in. ID) with a sintered stainless steel frit pressed tightly into the base. All frits had a nominal pore size of $0.5 \mu \mathrm{m}$.

The sample holder assembly is capped by an enclosure body and cap. The body is a 2.00-in.-OD, 1.86-in.-ID (0.07-in. wall thickness) cylinder approximately 5 inches tall with 3 -in.-diameter upper and lower flanges. The enclosure cap has a matching 3-in. flanged base and 1.86-in. initial internal diameter that gradually tapers down to a 0.19 -in.-ID outlet (0.295-in.-OD barb). To assemble the enclosure, the enclosure body is lowered over the sample holder, and the cap is placed on top of the enclosure body. Rubber O-rings are placed between the base and cap flanges to provide an air-tight seal, which is required to minimize outside air being drawn into the system without passing through the air-balance HEPA filter. The enclosure is sealed by tightly clamping the upper and lower enclosure flanges. Air balance is provided by two 0.25 -in.-OD, 0.19 -in.-ID balance ports connected to a single HEPA filter. It should be noted that with the exception of the Teflon ${ }^{\circledR}$ ferule, rubber o-rings, and HEPA filter cartridge, the sample holder assembly is constructed entirely from stainless steel.

HEPA filters are Whatman HEPA-Cap Disposable Air Filtration Capsule No. 75 and were obtained from Fisher Scientific (Waltham, MA 02454). This type of filter was employed because of its ability to filter a high capacity of air with a minimal pressure drop (1 psig at 150 standard liters per minute (SLM)). A low filter-pressure drop is required for proper operation of particle collection and analysis systems, which are designed for ambient pressure operation. The HEPA filters employed in the SAL FIATA serve to both filter input air and exhaust system air. HEPA filters \#1 and \#4 are system exhausts and serve to minimize the spread of spent-fuel particles into the hot cell. HEPA filters \#2 and \#3 are input filters that serve to remove ambient dusts and aerosols that could otherwise be mistaken for spent-fuel release from the balance air to the sample enclosure and particle collectors.

Two types of particle collection/analysis systems are employed: optical particle counters (OPCs) and cascade impactors (see Table 3.2 and Table 3.3). Model 61636 Sixteen Channel Particle Profilers were purchased from Met One Instruments, Inc. (Grants Pass, Oregon 97526). These systems count the number of particles entrained in a flowing stream of air using the principle of laser diffraction. The range of measurement spans 0.5 to $20 \mu \mathrm{m}$; particles greater than $20 \mu \mathrm{m}$ are lumped into a single oversize fraction. In addition, these particle profilers can be connected to a computer to provide a real-time readout of particle-release events. The Met One profile samples air at $1 \mathrm{~L} / \mathrm{min}$ and it has an internal flow loop that recirculates filtered air at $3 \mathrm{~L} / \mathrm{min}$. The OPCs were calibrated and validated by Met One Instruments, Inc. prior to delivery.

Sioutas Cascade Impactors with Leland Legacy ${ }^{\circledR}$ Pump Kits were obtained from SKC West Inc. (Fullerton, CA 92834-4133). The Sioutas Cascade Impactor is a four-stage collector that provides a rough sort (between 0.5 and $10 \mu \mathrm{m}$ ) of airborne particulate material generated by the FIATA flowassembly material. Particles larger than $10 \mu \mathrm{m}$ are collected on the first stage; particles smaller than 
$0.5 \mu \mathrm{m}$ are collected on a post-impactor filter. It should be noted that the cascade impactor is only a collector. The amount and size of material collected must be characterized using separate analytic techniques (such as dosimetry and scanning electron microscopy). The cascade impactors are accompanied by a Leland Legacy ${ }^{\circledR}$ Pump, which draws air through the impactors at flows of $9 \mathrm{~L} / \mathrm{min}$.

Table 3.2. Operational Parameters for Met-One Instruments, Inc., Model 61636 Sixteen-Channel Particle Profilers

\begin{tabular}{|l|l||}
\hline Principle of Operation: & Laser Diffraction \\
\hline Measuring range: & 0.5 to $20 \mu \mathrm{m}$ (16-channel analyzer). \\
\hline Air sampling rate: & $1 \mathrm{~L} / \mathrm{min}$ \\
\hline Result: & $\begin{array}{l}\text { Real-time particle counts for various size ranges falling between } 0.5 \\
\text { and } 20 \mu \mathrm{m} . \text { Post-measurement analysis converts these number } \\
\text { distributions into mass distributions. Particles over } 20 \mu \mathrm{m} \text { are } \\
\text { counted, but no size distribution is given. }\end{array}$ \\
\hline
\end{tabular}

Table 3.3. Operational Parameters for Sioutas Cascade Impactors

\begin{tabular}{|l|l||}
\hline Principle of Operation: & Separation Based on Particle Inertia \\
\hline Measuring range: & $\begin{array}{l}\text { A four-stage separation over } 0.5 \text { to } 10 \mu \mathrm{m} . \text { Particles greater than } \\
10 \mu \mathrm{m} \text { are collected on the first plate. Particles under } 0.5 \mu \mathrm{m} \text { are } \\
\text { collected on a final filtration stage. }\end{array}$ \\
\hline Air sampling rate: & $\begin{array}{l}\text { L/min } \\
\text { Result: }\end{array}$ \\
$\begin{array}{l}\text { size. The quantity of particles collected may be determined by mass- } \\
\text { difference measurements on the collection stages. Separate analysis } \\
\text { (such as SEM) may be run after collection to determine particle size. }\end{array}$ \\
\hline
\end{tabular}

The atomizer employed in testing is a Model 3079 Portable Atomizer Aerosol Generator from TSI Incorporated (Shoreview, MN 55126). In the current setup, it is used to verify the performance of the optical particle counters. Specifically, it is used to atomize (form an aerosol) monodisperse polystyrene latex (PSL) spheres from a PSL solution. The atomizer is capable of dispersing PSL spheres up to $3 \mu \mathrm{m}$ in diameter. For verifying the SAL FIATA, Standard Reference Material ${ }^{\circledR} 1690$ was obtained from NIST. SRM 1690 is a $0.5-\mathrm{wt} \%$ suspension of nominal $1-\mu \mathrm{m}$-diameter polystyrene spheres (certified number average diameters of $0.895 \pm 0.008 \mu \mathrm{m})$ in water.

All gas flow lines and valves external to the hot cell are constructed of stainless steel. Gas flow lines are $\frac{3}{8}$ in. OD. In the hot cell, a combination of stainless steel tubing and plastic/conductive tubing is used. Gas flow lines before the sample boat are constructed of $3 / 8$-in.-OD stainless steel. Supply lines are connected to the sample chamber with a 0.5-in.-OD stainless steel flexible line. All HEPA filters are connected to the system using 0.5 -in.-OD Tygon ${ }^{\circledR}$ tubing. Lines intended for particle transport, such as the line connecting the sample chamber or atomizer to particle collection systems (i.e., the OPC and cascade impactor), use conductive silicone tubing for particle transport (0.19-in. inner diameter) from TSI Incorporated (Shoreview, MN 55126). 


\subsection{Preparation of the Particle Collection/Analysis System}

The collection system employed for fuel-rod segment and powder testing was either a single OPC or cascade impactor. These systems had to be properly prepared before beginning work.

As per the manufacturer's recommended instructions, the optical particle counter must be "zero checked" before each series of planned measurements to verify that the optical system is free of particles. Zero checks involve testing HEPA-filtered air in the OPC and verifying that the instrument does not detect any particles in the filtered air for a given period of time (typically 5 minutes). During the hot-cell testing of the OPC, it was observed that the radiation fields generated by the highly radioactive spent-fuel rods and powders gave false particle detections on the $0.5-\mu \mathrm{m}$ channel. Specifically, when a 1.5-in. fuel rod segment was placed immediately next to the detector, the OPC measured approximately 1000 false $0.5-\mu \mathrm{m}$ particle counts per second as a result of the radiation. At a distance of $30 \mathrm{~cm}$, radiation counts fell to 10 to 30 counts per second. Shielding fuel elements and the OPC detector with lead bricks reduced the false counts to less than 2 counts per second but did not eliminate them entirely. As such, the zero check "pass" criterion was reduced to observing no greater than 2 counts per second over a 5-minute period. Anything lower was considered to be system background noise.

In addition to the zero check, a field-performance check of the OPC was performed before and after each series of measurements. Field-performance checks used a well-characterized material to verify that the instrument properly measures particle size. Specifically, the OPC was used to analyze the size distribution of a 1- $\mu \mathrm{m}$ PSL standard from NIST aerosolized by the atomizer. The OPC was considered to pass the performance check if all the following conditions were met: 1 ) the $50^{\text {th }}$-percentile diameter for the particle-size standard was greater than 0.9 times the lower bounding diameter on the bin showing the highest particle counts (differential-particle-size histogram), and 2) the $50^{\text {th }}$-percentile diameter for the particle-size standard was less than 1.1 times the upper bounding diameter on the bin showing the highest particle counts (differential-particle-size histogram).

Preparing cascade impactors involved replacing the filters on each stage. Because of the difficulties associated with working with spent fuel outside of a shielded facility, a new cascade impactor was used for each measurement. Each new cascade impactor was disassembled before being installed in the hot cell. Clean Teflon ${ }^{\circledR}$ filters were installed on each of the four collection stages and a post-impactor, deadend Teflon filter was installed to collect any particles that managed to pass through the previous stages of the impactor. Once the filters were in place, the impactor was reassembled and moved into the hot cell for testing.

\subsection{Post-Measurement Operations}

After each segment/oxidized powder test, the OPC or cascade impactor pump was powered down. The conductive tubing connecting the sample chamber to the particle collection system was removed, rolled-up, and placed into a clean, labeled plastic bag for post-measurement estimates of particle hold-up (using dosimetry). The sample enclosure was disassembled and the fuel rod segment or sample boat removed and set aside. The interior surfaces of the sample enclosure were inspected and any large fragments of fuel or powder aggregations on the interior surfaces of the enclosure were collected (and in 
the case of powder samples, returned to the sample boat). Once all large fragments were collected, the interior surfaces of the enclosure were wiped down with a damp cloth. All wipes were collected in a separate plastic bag for post-analysis dosimetry.

For tests using the OPC, raw measurement data were downloaded from the counter to a computer for post-measurement analysis. The OPC particle count logs were supplemented by the time log for flow setpoint/mechanical action that was recorded during analysis. For tests using the cascade impactor, the impactor was placed in a plastic bag and loaded out of the hot cell for post-measurement dosimetry.

After all planned measurements were completed, a post-measurement performance check was conducted to verify continued OPC performance.

\subsection{Estimate of Release in Optical-Particle-Counter Tests}

Raw test data for optical particle counters is contained in two separate files: 1) the event data file and 2) the OPC count log. The event file is a simple record of the time that a set-point increase or a mechanical action (hereafter called an "event") took place. For example, an event-file log entry might read "Time: 5/25/2007 9:00:06, FC1: 30, FC2: 0, Action: Increased flow." This indicates that at 9:00:06 AM on May 25 $5^{\text {th }}, 2007$, the set point for flow controller FC1 was increased to 30 SCCM. The OPC count log contains the cumulative oversize counts for each particle bin at 1-second intervals for the duration of the measurement period. Each entry has an associated time, record number, counts for each of the 16 size channels, and information regarding the OPC operational status. In their raw format, counts for particle size are presented as cumulative oversize counts for the sizes shown in Table 3.4:

Table 3.4. Particle-Size Settings for the 16-Channel OPC

\begin{tabular}{||c|c||c|c||}
\hline Bin Number & Size Range $(\boldsymbol{\mu m})$ & Bin Number & Size Range $(\mu \mathrm{m})$ \\
\hline 1 & $>0.5$ & 9 & $>11.0$ \\
2 & $>0.7$ & 10 & $>12.0$ \\
3 & $>1.0$ & 11 & $>13.0$ \\
4 & $>2.0$ & 12 & $>14.0$ \\
5 & $>5.0$ & 13 & $>15.0$ \\
6 & $>7.0$ & 14 & $>16.0$ \\
7 & $>9.0$ & 15 & $>18.0$ \\
8 & $>10.0$ & 16 & $>20.0$ \\
\hline
\end{tabular}

The OPC count log can be expressed as a two-dimensional array consisting of $\mathrm{M}$ total entries. Each OPC count $\log$ entry $j$ is of the form:

$$
\left\langle t^{(j)}, N_{1}^{(j)}, N_{2}^{(j)}, \ldots N_{i}^{(j)} \ldots, N_{16}^{(j)}\right\rangle \text { for } j=1 \text { to } M
$$

Here $t$ is time and $N_{i}$ is the cumulative oversize count for size bin $i$. 
The first step in the analysis was to convert all cumulative oversize counts into differential counts for each size bin (i.e., the number of particles falling between the diameters associated with the bins). This is done through the following equation:

$$
n_{i}^{(j)}=N_{i}^{(j)}-N_{i+1}^{(j)}
$$

where $n_{i}{ }^{(j)}$ is the number of differential particle counts in the $i^{\text {th }}$ bin in count log entry $j$. It should be noted that $\mathrm{N}_{\mathrm{i}+1}$ does not exist for bin 16 (hereafter referred to as the "oversize" bin). In this case, the differential count is simply:

$$
n_{16}^{(j)}=N_{16}^{(j)}
$$

The size range corresponding to each bin calculated using this method is as shown in Table 3.5:

\begin{tabular}{|c|c|c|}
\hline Bin Number & Size Range $(\mu \mathrm{m})$ & Average Size $(\mu \mathrm{m})$ \\
\hline 1 & $0.5-0.7$ & 0.65 \\
\hline 2 & $0.7-1.0$ & 0.85 \\
\hline 3 & $1.0-2.0$ & 1.5 \\
\hline 4 & $2.0-5.0$ & 3.5 \\
\hline 5 & $5.0-7.0$ & 6.0 \\
\hline 6 & $7.0-9.0$ & 8.0 \\
\hline 7 & $9.0-10.0$ & 9.5 \\
\hline 8 & $10.0-11.0$ & 10.5 \\
\hline 9 & $11.0-12.0$ & 11.5 \\
\hline 10 & $12.0-13.0$ & 12.5 \\
\hline 11 & $13.0-14.0$ & 13.5 \\
\hline 12 & $14.0-15.0$ & 14.5 \\
\hline 13 & $15.0-16.0$ & 15.5 \\
\hline 14 & $16.0-18.0$ & 17.0 \\
\hline 15 & $18.0-20.0$ & 19.0 \\
\hline 16 & $>20.0$ & $20(a)$ \\
\hline \multicolumn{3}{|c|}{$\begin{array}{l}\text { No average diameter is defined for the oversize bin. A } \\
\text { value of } 20 \mu \mathrm{m} \text { is taken for use in subsequent calculations }\end{array}$} \\
\hline
\end{tabular}

Table 3.5. Average Particle Size for each OPC Channel

The next step in analyzing the OPC data is to match up the events listed in the event log to the data in the OPC count log. This is accomplished by defining an event period for each event that spans the start of the current event to the start of the next event. For example, the event period for event $k$ is bound by the start time for that event, $t^{(a)}$, and the start of event $k+1, t^{(b)}$. Here, $j=a$ and $j=b$ are the OPC count entry rows corresponding to times $t^{(a)}$ and $t^{(b)}$.

Once the period is determined for each recorded event, the number of particles observed during that release event and their distribution can be determined. For event $k$, the total number of particles observed in size bin $i$, given by $\eta_{\mathrm{i}}{ }^{(\mathrm{k})}$, is 


$$
\eta_{i}^{(k)}=\sum_{j=a}^{b} n_{i}^{(j)}
$$

The total number release within the operational range of the OPC (i.e., $0.5-20 \mu \mathrm{m}), \mathrm{H}^{(k)}$, is given by,

$$
\mathrm{H}^{(k)}=\sum_{i=1}^{15} \eta_{i}^{(k)}
$$

The total number release over all bins (including the oversize bin), $\mathrm{H}_{\text {tot }}{ }^{(k)}$, is:

$$
\mathrm{H}_{\text {tot }}^{(k)}=\sum_{i=1}^{16} \eta_{i}^{(k)}
$$

From this, a number distribution of particles may be calculated for event $k$ :

$$
f_{i}^{(k)}=\frac{\eta_{i}^{(k)}}{\mathrm{H}^{(k)}}, \text { for } \mathrm{i}=1 \text { to } 15
$$

After the number distribution is calculated, estimates of the release volume of particles and the volume distribution can be made. For event $k$, the volume of particles in bin $i$ is given by:

$$
v_{i}^{(k)}=\frac{\pi \eta_{i}^{(k)} d_{i}^{3}}{6}
$$

where $d_{i}$ is the average size range for bin $i$. Then, the total volume release within the operational range of the OPC, $V^{(k)}$, and the total volume release over all bins, $V_{\text {tot }}{ }^{(k)}$, are, respectively:

$$
\begin{aligned}
& V^{(k)}=\sum_{i=1}^{15} v_{i}^{(k)} \\
& V_{t o t}^{(k)}=\sum_{i=1}^{16} v_{i}^{(k)}
\end{aligned}
$$

The volume distribution of particles for event $k$ is then calculated as:

$$
x_{i}^{(k)}=\frac{v_{i}^{(k)}}{V^{(k)}}, \text { for } \mathrm{i}=1 \text { to } 15
$$

Finally, an estimate of powder mass released as measured by the OPC, $m_{\text {release }}^{(k)}$, can be made by multiplying the total volume released by the material density. That is, 


$$
m_{\text {release }}^{(k)}=\rho V_{\text {tot }}^{(k)}
$$

where $\rho$ is the material density.

In addition to the differential number and volume distributions described above, results can also be expressed as cumulative undersize distributions. Cumulative distributions are calculated as:

$$
\begin{gathered}
\hat{\eta}_{l}^{(k)}=\sum_{i=1}^{l} \eta_{i}^{(k)}, \text { and } \\
\hat{x}_{l}^{(k)}=\sum_{i=1}^{l} x_{i}^{(k)}
\end{gathered}
$$

where $\hat{\eta}_{l}^{(k)}$ and $\hat{x}_{l}^{(k)}$ are the cumulative undersize distributions for particle number and volume, respectively, of the $l^{\text {th }}$ bin during event $k$. The cumulative volume distribution allows particle diameters to be calculated corresponding to the $10^{\text {th }}, 50^{\text {th }}$, and $90^{\text {th }}$ percentiles via:

$$
d(w)^{(k)}=\left(\frac{d_{m+1}-d_{m}}{\hat{x}_{m+1}^{(k)}-\hat{x}_{m}^{(k)}}\right)\left(w-\hat{x}_{m}^{(k)}\right)+d_{m}
$$

In this equation, $w$ is the desired percentile (i.e., 10, 50, or 90). The points $m$ and $m+1$ correspond to the cumulative oversize fractions, $\hat{x}_{m}^{(k)}$ and $\hat{x}_{m+1}^{(k)}$, that bound the desired percentile and have associated average sizes of $d_{m}$ and $d_{m+1}$.

The analysis outlined above is performed on all events listed in the OPC event log using the data in the OPC particle-counts log. The end result is a matrix including the particle counts, mass, and mass/volume distributions associated with each event. The final step in the analysis was to differentiate between measurements showing significant particle release and those showing less than background. This step is necessary only because incident radiation from the fuel rod segments and powders gave occasional false peaks on the optical particle counter. It was observed that with the sample enclosure located at a distance of approximately 3 feet from the OPC and with lead brick shielding over the OPC detector, background counters were reduced to less than 2 counts per second. As such, it was determined whether an event $k$ showed significant release as follows:

1. The maximum differential count observed during even $k$ is determined through

$$
n_{\max }^{(k)}=\operatorname{Maximum}\left[n_{i}^{(j)}\right] \text { for } \mathrm{i}=1 \text { to } 16, \mathrm{j}=\mathrm{a} \text { to } \mathrm{b}
$$

It should be noted that since each log file entry row $j$ corresponds to a 1 -second interval, the maximum counts determined by the above equation also correspond to the maximum release rate (in particles per second). 
2. If $n_{\max }^{(k)} \geq 3$, release for event $k$ is considered significant and reportable. On the other hand, if $n_{\max }^{(k)}<3$, then release for event $\mathrm{k}$ is considered to be below background and is not reported.

In the results sections that follow, only those particle release events showing greater than background concentrations of particles are reported.

\subsection{Estimates of Mass Release and Release Fraction}

Fuel release during rod segment and oxidized powder tests is defined by the amount of material leaving the rod segment or sample boat. However, because of the constraints related to disassembling the SAL FIATA, the only real time, event-by-event estimate of release is provided by the OPC measurements. As discussed in detail in Section 6, OPC detection is not $100 \%$ efficient in that not all of the sample material makes it to the detector. Indeed, sample loss occurs as a result of sticking and settling of particles entrained in the air flow and as a result of splitting of the flow stream at flows greater than 1 SLM (see Section 6 for additional discussion). Given an appropriate counting efficiency, the actual material released from the sample boat/rod may be estimated from that detected alone. Specifically, the actual sample boat/rod release occurring during an event $k, m_{\text {actual }}^{(k)}$, is given by:

$$
m_{\text {actual }}^{(k)}=\frac{m_{\text {release }}^{(k)}}{\phi_{o}}
$$

where $\phi_{o}$ is an estimate of the global counting efficiency. Based on the results in Section 6, a reasonable estimate of the global efficiency is $\phi_{o} \sim 0.065$. Then, the release fraction (F) for event $k$, which is defined as the ratio of mass released to mass available for release, can be determined from the equation:

$$
F=\frac{m_{\text {actual }}^{(k)}}{m_{\text {sample }}}
$$

Here, $m_{\text {sample }}$ is the mass of the sample given. For oxidized fuel powders, it is simply the mass of powder placed in the sample boat. For spent-fuel rod segments, it corresponds to the amount of fuel material in the entire length of rod. 


\subsection{Execution of Fuel Rod Segment Tests}

Experimental studies for fuel rod segment release tests employed the SAL FIATA setup described in the preceding sections. Four different commercial spent-fuel rod segments were tested:

- ATM-106 - OPC only (3 tests)

- HBU 539B3 - Cascade Impactor (1 test) and OPC (3 tests)

- HBU 539D5 - Cascade Impactor (1 test) and OPC (1 test)

- HBU 539F5 - OPC only (2 tests)

The ATM-106 fuel, which is from a PWR, achieved a burnup of approximately $45 \mathrm{GWd} / \mathrm{MTU}$ and is characterized in Guenther et al. (1988b). The three HBU fuels are high burnup BWR fuels and achieved burnups in the range $53 \mathrm{GWd} / \mathrm{MTU}$ to $69 \mathrm{GWd} / \mathrm{MTU}$ and are characterized by Vaidyanathan et al. (1997). These BWR fuels are known as barrier fuels in that there was a liner of $\mathrm{Zr}$ on the fuel-side of the Zircaloy cladding.

Segment tests were conducted by securing the fuel segment (see Table 4.1 for additional information) in the sample holder. The sample enclosure was then assembled and connected to the particle collection systems using a clean, 3-foot section of conductive tubing. It should be noted that 3 feet of conductive tubing is the maximum recommended for particle transport by the manufacturer (TSI). The length of tubing was selected to maximize the distance between the OPCs and the highly radioactive fuel segment, reducing the number of false particle counts on the OPC as a result of radioactivity.

Table 4.1. Estimated Dimensions and Mass of Fuel Rod Segments Used in SAL FIATA Testing

\begin{tabular}{|l|c|c|c|c||}
\hline & ATM-106 & HBU 539B3 & HBU 539D5 & HBU 539F5 \\
\hline Dimensions: & & & & \\
Outer Diameter: & $1.118 \mathrm{~cm}^{(\mathrm{a})}$ & $\sim 1.23 \mathrm{~cm}$ & $\sim 1.23 \mathrm{~cm}$ & $\sim 1.23 \mathrm{~cm}$ \\
Inner Diameter: & $0.986 \mathrm{~cm}^{(\mathrm{a})}$ & $\sim 1.11 \mathrm{~cm}$ & $\sim 1.11 \mathrm{~cm}$ & $\sim 1.11 \mathrm{~cm}$ \\
Pellet Diameter: & $0.9639 \mathrm{~cm}^{(\mathrm{a})}$ & $(\mathrm{b})$ & $(\mathrm{b})$ & $(\mathrm{b})$ \\
Length: & $3.65 \mathrm{~cm}$ & $5.08 \mathrm{~cm}$ & $4.92 \mathrm{~cm}$ & $5.08 \mathrm{~cm}$ \\
\hline Mass: & $\mathrm{NA}$ & $55.2753 \mathrm{~g}$ & $52.8093 \mathrm{~g}$ & $54.8246 \mathrm{~g}$ \\
$\quad$ Total & $26.6 \mathrm{~g}$ & $49.2 \mathrm{~g}$ & $47.6 \mathrm{~g}$ & $49.2 \mathrm{~g}$ \\
$\quad$ Fuel & \multicolumn{4}{|c||}{} \\
\hline (a) Pre-irradiation dimension. & \\
(b) Assumed to be the same as the inner diameter since the pellet/gap closes at high burnup. \\
(c) Calculated using pellet diameter, length, and an assumed density of $10 \mathrm{~g} / \mathrm{cc}$.
\end{tabular}

Once the sample holder was assembled and connected to the particle collection system, the particle collection/analysis systems and associated pumps were powered on. At this point, all air flow through the sample chamber was being drawn in through the balance air ports by the particle collection system. For experiments with the OPC, a zero check was performed to verify that the system was sealed and that all balance air drawn into the system was being drawn through the HEPA filters. After a successful OPC zero check, the clocks on the computer controlling the OPC and the internal clock on the OPC were synchronized. 
After all equipment was in a measurement-ready state, the gas supply bottle was opened and regulated to a pressure above that needed to overcome the 10-psig check valve. Valves V1 and V3 were opened and flows ranging from 0 to 200 SCCM were tested using flow-controller FC1. At 200 SCCM, the flow control was given to controller FC2 by closing valve V1 and opening valve V2. Flow controller exchange often led to a 1- to 2-second spike in flow rate. Gas flow rates from 200 SCCM to 9 SLM were tested using FC2. In many cases, the regulated gas pressure had to be increased to achieve the desired flow rate. For fuel segments HBU 539B3 and 539D5, the maximum system pressure (130 psig - flow controller safety limit) was met at gas flow rates below 500 SCCM. As such, the high-end flow rates could not be tested for these systems.

At high flow rates, typically 3 SLM or beyond, the fuel segments were subjected to the following two different mechanical actions to facilitate particle release:

- Tapping - the enclosure body, cap, or inlet lines were tapped with a wrench located in the hot cell. In some instances, the conductive tubing between the sample chamber and particlecollection system was shaken. Tapping and shaking operations were each of 30 seconds in duration or less.

- Pressure pulsing - valve V3 was closed and the cell side of the system was allowed to depressurize. Valve V3 was then quickly opened, resulting in a rapid increase in cell-side pressure.

For fuel segments HBU 539B3 and 539D5, tapping and pressure pulsing were done at or near the maximum flow rate achieved. Mechanical agitation was only done on measurements where the release could be directly observed and correlated to events causing them (i.e., those measurements employing optical particle counters). Mechanical agitation was not performed during measurement with cascade impactors.

Throughout each fuel rod experiment, the time of each flow rate increase and/or mechanical action was recorded so that each observed particle release could be correlated to a change in conditions. For measurements employing cascade impactors, set points were held for at least 3 minutes and typically for longer periods of time. For measurements using OPC, release events were monitored in real time through a computer connection to the particle analysis system. Particle counts were measured and recorded at a frequency of $1 \mathrm{~Hz}$. Typically, set points were held until the particle release caused by the last action fell below background. In cases where sustained particle release occurred for greater than 30 minutes, the next flow condition/action was set without waiting for counts to fall to background levels.

All fuel rod segments were tested more than once. Each "repeat" measurement was made on the same segment as the previous test. All rod release tests consist of at least one OPC measurement. Only fuel segments HBU 539B3 and 539D5 employed cascade impactors.

Repeat OPC measurements for ATM-106 and HBU 539B3 examined the influence of rod segment orientation. In particular, ATM-106 tests 1 and 3 use a "C up" orientation, whereas test 2 uses a "C down" orientation. The orientation terminology refers to the faint " $\mathrm{C}$ " impression that can be seen on the ATM-106 segment end shown in Figure 4.1b. In the "C up" orientation, the segment end shown in Figure 4.1a is secured in the sample holder's Swagelok ${ }^{\circledR}$ fitting while the end shown in Figure 4.1b is exposed to the sample chamber. In this orientation, gas flows into end $4.1 \mathrm{a}$ and out of end $4.1 \mathrm{~b}$. In the 
"C down" orientation, the rod is reversed such that gas flows into end $4.1 \mathrm{~b}$ (which is secured in the sample holder) and out of 4.1a.

OPC tests 1 and 3 for HBU 539B3 use a "plug up" orientation. Test 2 uses a "plug down" orientation. The "plug" terminology refers to the dark semi-circular area seen on the exposed fuel end in Figure 4.1d. In the "plug up" orientation, the end shown in Figure 4.1c is secured in the sample holder; the end shown in Figure 4.1d is exposed to the sample chamber. Gas flows into end 4.1c and out of 4.1d. As before, the ends are reversed in the "plug down" orientation.

\subsection{Overall Results of CSNF Rod Segment Tests}

Summary results of the CSNF rod segment tests are presented in Table 4.2 and Table 4.3. Table 4.2 gives a rough outline of the flow-rate ranges and mechanical actions each fuel rod was subjected to during testing. Repeat measurements are listed separately; for example, the second test with the ATM106 rod is designated "ATM106 (2/3)." The flow-rate range for fuels HBU 539B3 and 539D5 were limited by the maximum allowable system pressure $(130 \mathrm{psig})$. This suggests that these fuels have little internal pore space for gas flow. Table 4.1 shows both ends of each of the four rods tested. While a clear fuel/clad gap is observed with the ATM-106 fuel, which would account for the high flow rates achieved, similar gaps and distinguishable cracks are missing from the HB fuels. The phenomenon of fuel/clad gap closure at higher burnups is well documented. Initial testing of fuel HUB 539F5 achieved the entire range of flow rates; however, repeat testing found that flow was limited to 0.75 SLM. This suggests a change in the fuel configuration during testing, as a result of either fuel relocation or a difference in the way the fuel was secured in the sample holder.

Table 4.3 shows the amount and characteristics of fuel particulates detected at the OPC. It includes

the raw-particle counts, an estimate of the volume of spent fuel released, and the PSD in terms of the $10^{\text {th }}$, $50^{\text {th }}$, and $90^{\text {th }}$ diameter percentiles for all events and mechanical actions performed on the listed fuel rod segment. 

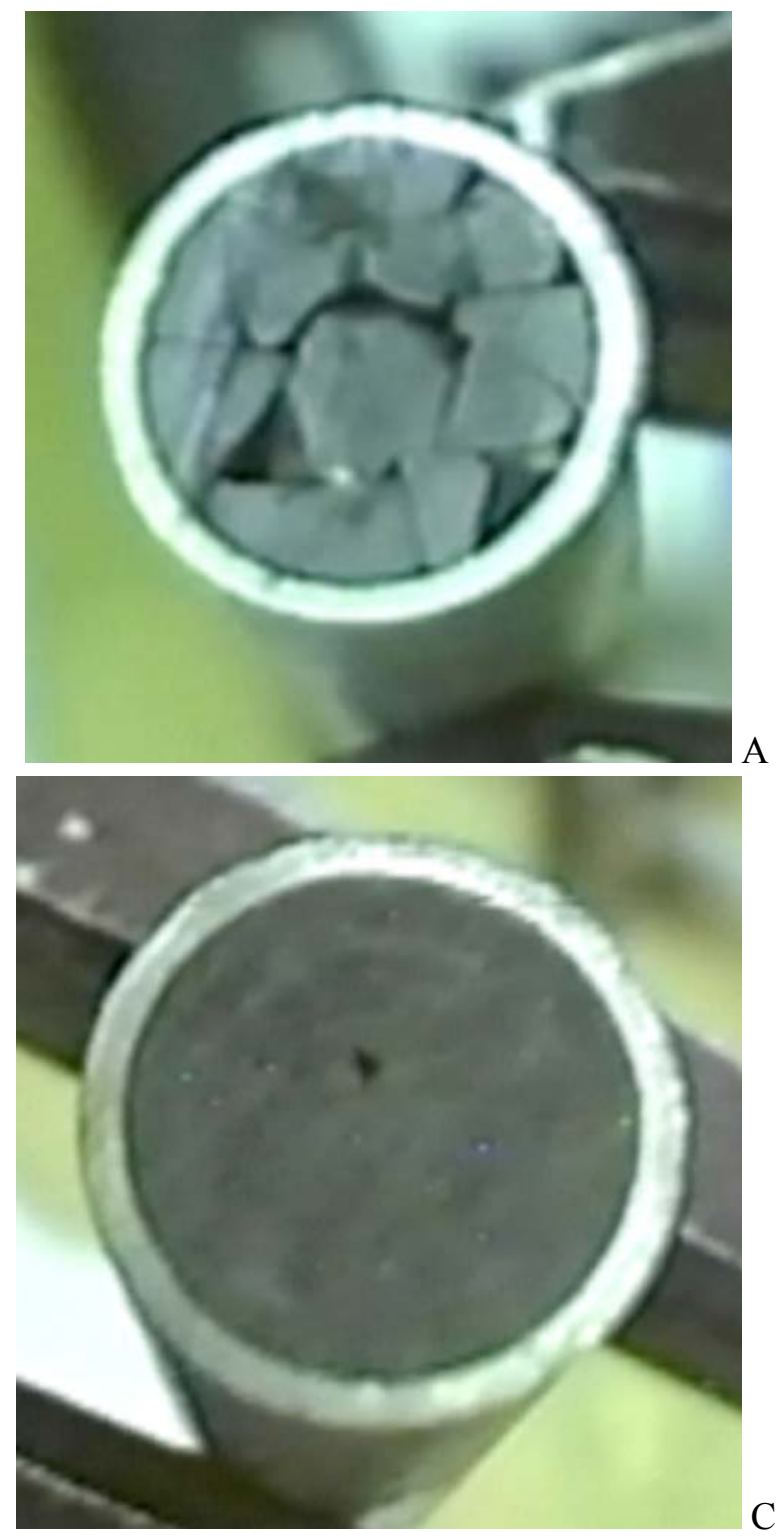
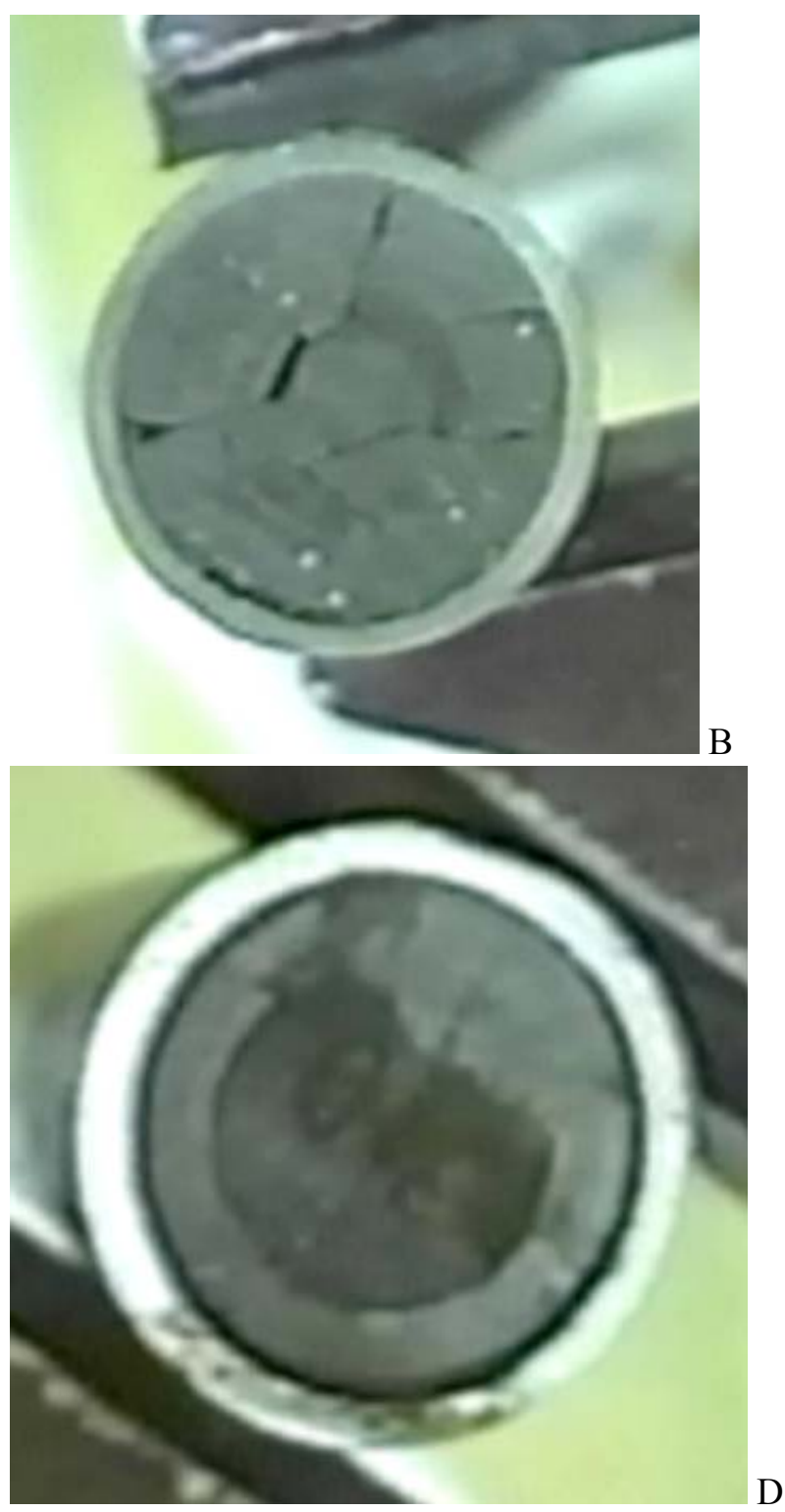

Figure 4.1. Photographs of each End of the Four CSNF Rod Segments Tested: $a-b)$ ATM-106, c-d) HBU 539B3, e-f) HBU 539D5, g-h) HBU539F5 

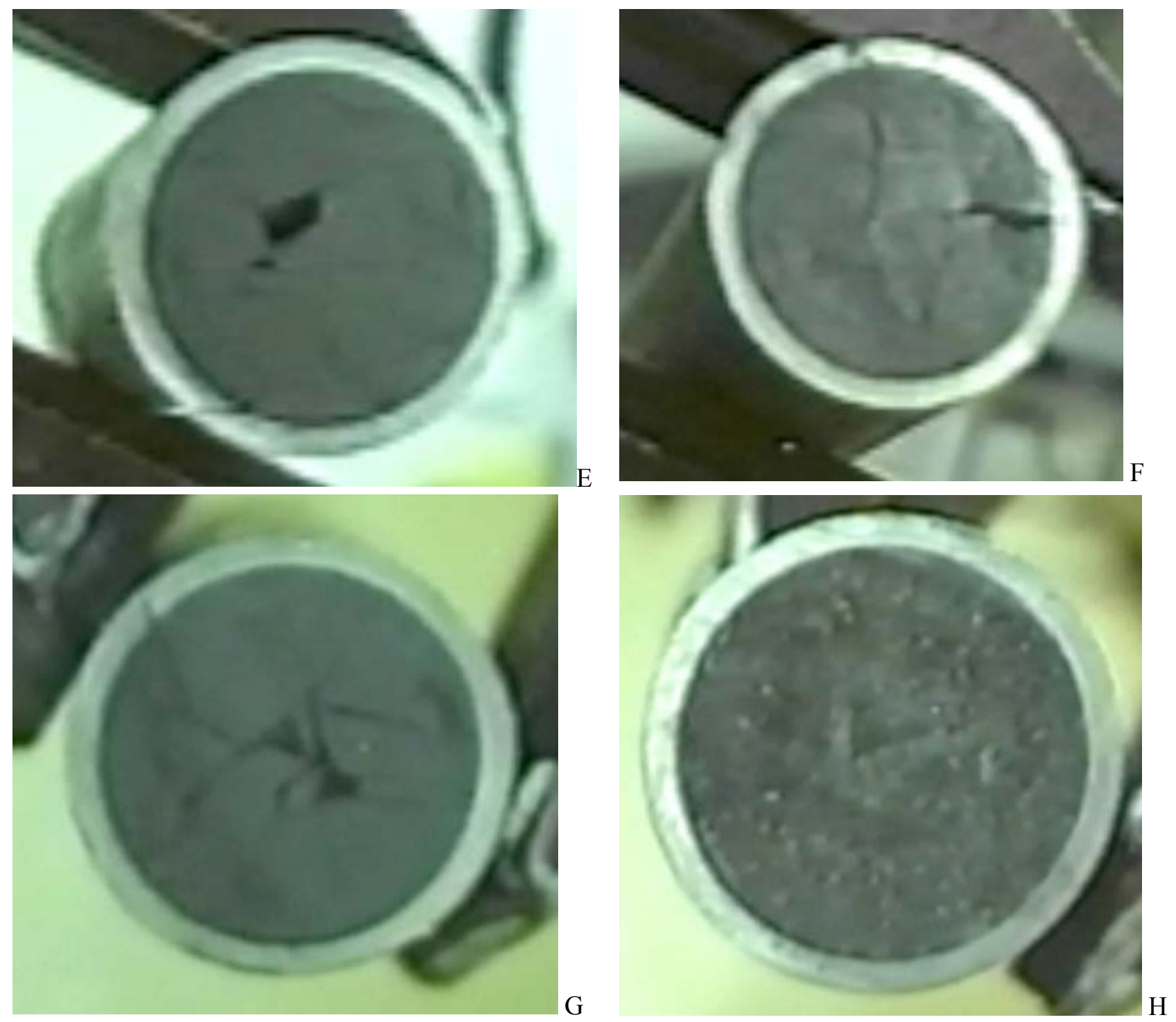

Figure 4.1 (Contd) 
Table 4.2. Summary of CSNF Rod Segment Testing Parameters

\begin{tabular}{|c|c|c|c|c|}
\hline System & Duration [s] & $\begin{array}{c}\text { Flow Rate } \\
\text { Range }[\text { SLM] }\end{array}$ & Pressure Pulses & $\begin{array}{l}\text { Tapping/Shaking } \\
\text { Operations }\end{array}$ \\
\hline ATM106 (1/3) & 3078 & $0.01-9.0$ & 19 & 4 \\
\hline ATM106 (2/3) & 7596 & $0.01-9.0$ & 2 & 4 \\
\hline ATM106 (3/3) & 6072 & $0.01-9.0$ & 3 & 4 \\
\hline HBU 539B3 (1/3) & 7380 & $0.01-0.37^{(\mathrm{a})}$ & 3 & 2 \\
\hline HBU 539B3 (2/3) & 5850 & $0.01-0.35^{(\mathrm{a})}$ & 2 & 2 \\
\hline HBU 539B3 (3/3) & 4646 & $0.01-0.36^{(\mathrm{a})}$ & 2 & 4 \\
\hline HBU 539D5 & 5861 & $0.01-0.48^{(\mathrm{a})}$ & 4 & 1 \\
\hline HBU 539F5 (1/2) & 4032 & $0.01-9.0$ & 10 & 9 \\
\hline HBU 539F5 (2/2) & 4712 & $0.01-0.75^{(\mathrm{a})}$ & 1 & 4 \\
\hline \multicolumn{5}{|c|}{$\begin{array}{l}\text { (a) Indicates a test where the flow-rate range was limited by the maximum allowable system } \\
\text { pressure ( } 150 \text { psig). }\end{array}$} \\
\hline
\end{tabular}

Table 4.3. Summary of CNSF Rod Segment Test Release Results

\begin{tabular}{||l|c|c|c|c|c||}
\hline \multicolumn{1}{|c|}{ System } & $\begin{array}{c}\text { Counts } \\
{[\text { particles] }}\end{array}$ & $\begin{array}{c}\text { Volume } \\
\text { Released }\left[\mathbf{c m}^{3}\right]\end{array}$ & $\begin{array}{c}\mathbf{d 1 0} \\
{[\mu \mathbf{m}]}\end{array}$ & $\begin{array}{c}\mathbf{d 5 0} \\
{[\mu \mathbf{m}]}\end{array}$ & $\begin{array}{c}\mathbf{d 9 0} \\
{[\mu \mathbf{m}]}\end{array}$ \\
\hline ATM106 (1/3) & $2.6 \times 10^{5}$ & $1.9 \times 10^{-6}$ & 1.7 & 4.5 & 14.5 \\
\hline ATM106 (2/3) & $8.5 \times 10^{6}$ & $1.4 \times 10^{-5}$ & 0.9 & 2.2 & 3.9 \\
\hline ATM106 (3/3) & $6.0 \times 10^{6}$ & $8.0 \times 10^{-6}$ & 0.8 & 2.2 & 6.9 \\
\hline $\begin{array}{l}\text { HBU 539B3 } \\
(1 / 3)\end{array}$ & $1.4 \times 10^{5}$ & $2.9 \times 10^{-7}$ & 1.0 & 3.1 & 10.4 \\
\hline $\begin{array}{l}\text { HBU 539B3 } \\
(2 / 3)\end{array}$ & $3.8 \times 10^{5}$ & $1.5 \times 10^{-6}$ & 1.2 & 2.9 & 13.4 \\
\hline $\begin{array}{l}\text { HBU 539B3 } \\
(3 / 3)\end{array}$ & $1.6 \times 10^{5}$ & $1.4 \times 10^{-6}$ & 2.0 & 8.6 & 16.8 \\
\hline HBU 539D5 & $7.5 \times 10^{5}$ & $1.5 \times 10^{-6}$ & 1.0 & 2.3 & 4.6 \\
\hline $\begin{array}{l}\text { HBU 539F5 } \\
(1 / 2)\end{array}$ & $7.6 \times 10^{5}$ & $5.2 \times 10^{-6}$ & 1.5 & 2.9 & 11.6 \\
\hline $\begin{array}{l}\text { HBU 539F5 } \\
(2 / 2)\end{array}$ & $1.1 \times 10^{6}$ & $2.6 \times 10^{-6}$ & 1.0 & 2.4 & 5.2 \\
\hline
\end{tabular}

\subsection{Individual Results of CSNF Rod Segment Tests}

The overall release results given in Section 4.1 represent the sum of particle release as a result of each flow set point change and mechanical action. While this information is useful in gauging the physical properties of the fuel, such as the propensity to release particles relative to one another and the average size distribution of particles reaching the detector, it provides little information on how flow conditions affect particle release.

In the following subsections, particle release as a function of flow conditions and mechanical action will be provided. As indicated by Table 4.2 and discussed in Section 3, the times corresponding with 
each flow set point change, housing, etc, were recorded to allow direct correlation with OPC detection curves. However, fuel rod tests involved anywhere from 20 to 80 individual "events," and as such, it is impractical to list the release associated with each event in the current report. While an event-by-event breakdown of particle release is provided in Appendix A, the results presented in this section will examine release over four flow rate/agitation regimes. For ATM-106 segment tests, the aggregate conditions considered are:

- between 0 to 500 SCCM with no mechanical action

- between 500 to 1000 SCCM with no mechanical action

- between 1000 to 3000 SCCM with no mechanical action

- 3000 SCCM and above with mechanical.

Aggregate results for the remaining fuel rods follow a similar format. However, results for HBU 539B3 and HBU 539D5 are limited to only two conditions by the fact that the maximum obtainable flow rates for these rods was $\sim 400 \mathrm{SCCM}$ and $\sim 500 \mathrm{SCCM}$, respectively. For HBU 539F5 Test 1 of 2, mechanical actions were performed on the rod at 500 SCCM, and an additional aggregate condition has been added to reflect this.

\subsubsection{ATM-106 Rod Segment Test Results}

Table 4.4 through Table 4.6 list the results of the three rod segment tests for ATM-106. The estimated mass releases given in the tables are based on an assumed fuel density of $10 \mathrm{~g} / \mathrm{cc}$ and an OPC detection efficiency of 0.065 (see Section 6.3 .8 for details). Repeat tests were conducted in the order in which they are numbered and employ the same fuel-rod segment. As noted in the introduction to Section 4, tests 1 and 3 use a "C up" orientation, whereas test 2 uses a " $\mathrm{C}$ down" orientation. Note that because of rounding, the totals in some of the tables will appear to be incorrect. For consistency, all values are taken from the associated spreadsheets and then rounded to the appropriate number of significant figures in this document.

Table 4.4. Mass and Fractional Release for ATM-106 Rod Segment Test 1 of 3

\begin{tabular}{|l|c|c||}
\hline Region & $\begin{array}{c}\text { Estimated Mass } \\
\text { Release }[\boldsymbol{\mu g}]\end{array}$ & Release Fraction \\
\hline Release over 0 to 500 SCCM & 5 & $1.9 \times 10^{-7}$ \\
\hline Release over 500 to 1000 SCCM & 0 & 0 \\
\hline Release over 1000 to 3000 SCCM & 0 & 0 \\
\hline Release at 3000 SCCM and above, with mechanical action & 283 & $1.1 \times 10^{-5}$ \\
\hline Total & 288 & $1.1 \times 10^{-5}$ \\
\hline
\end{tabular}

Table 4.5. Mass and Fractional Release for ATM-106 Rod Segment Test 2 of 3

\begin{tabular}{|l|c|c||}
\hline Region & $\begin{array}{c}\text { Estimated Mass } \\
\text { Release }[\boldsymbol{\mu g}]\end{array}$ & Release Fraction \\
\hline Release over 0 to 500 SCCM & 529 & $2.0 \times 10^{-5}$ \\
\hline Release over 500 to 1000 SCCM & 426 & $1.6 \times 10^{-5}$ \\
\hline Release over 1000 to 3000 SCCM & 550 & $2.1 \times 10^{-5}$ \\
\hline
\end{tabular}




\begin{tabular}{|l|r|r||}
\hline \hline Release at 3000 SCCM and above, with mechanical action & 599 & $2.3 \times 10^{-5}$ \\
\hline Total & 2103 & $7.9 \times 10^{-5}$ \\
\hline
\end{tabular}

Table 4.6. Mass and Fractional Release for ATM-106 Rod Segment Test 3 of 3

\begin{tabular}{|l|c|c||}
\hline Region & $\begin{array}{c}\text { Estimated Mass } \\
\text { Release }[\boldsymbol{\mu g}]\end{array}$ & Release Fraction \\
\hline Release over 0 to 500 SCCM & 76 & $2.9 \times 10^{-6}$ \\
\hline Release over 500 to 1000 SCCM & 146 & $5.5 \times 10^{-6}$ \\
\hline Release over 1000 to 3000 SCCM & 310 & $1.2 \times 10^{-5}$ \\
\hline Release at 3000 SCCM and above, with mechanical action & 697 & $2.6 \times 10^{-5}$ \\
\hline Total & 1228 & $4.6 \times 10^{-5}$ \\
\hline
\end{tabular}

As shown by the results in Table 4.4 to Table 4.6, release results vary from test to test. In test 1 , only $5 \mu \mathrm{g}$ of fuel release is observed over flow rates spanning 0 to 3000 SCCM. Indeed, the most significant release, $283 \mu \mathrm{g}$, occurred at $3000 \mathrm{SCCM}$ and above with mechanical tapping. This contrasts strongly with the second test, which observed a relatively constant release of $\sim 500 \mu \mathrm{g}$ over each of the four flow regions examined and a total release of $2103 \mu \mathrm{g}$. While the $1228 \mu \mathrm{g}$ release observed in test 3 is the same order of magnitude, the release appears to be a strong function of flow region.

The differences outlined in the preceding paragraph appear to result from a combination of orientation and history effects. The high release results of test 2, compared to tests 1 and 3, suggest that the "C down" orientation (the " $\mathrm{C}$ " is the dark ring seen in Figure 4.1B) is more favorable to particle release. This is supported by visual inspection of the rod segment. Specifically, Figure 4.1a shows that the fuel at the exposed end in the "C down" orientation appears to be quite fractured with gaps and cracks that are easily visible without the aid of a microscope. Particles entrained upstream of this structure would be less likely to become trapped in the relatively open void structure. In comparison, the exposed fuel end in the "C up" orientation used in tests 1 and 3 shows less fragmentation and smaller cracks. This is consistent with the lessened release from this orientation. As such, it appears that fuel orientation does influence the observed release fraction.

Results from tests employing the same fuel orientation show substantial variation. The release in test 3 is approximately four times higher than that in test 1 . In addition, no fuel release was observed in the 500- to 3000-SCCM region in test 1, whereas test 3 saw release results comparable (but somewhat less) than in test 2 . The increased release in test 3 could suggest changes to the fuel-rod microstructure that favor particle release. It is postulated that mechanical actions performed during the course of testing opened up some of the fractures within the fuel pellet sufficiently to allow particle release. This is supported by the observation that no substantial release occurred in test 1 until mechanical action was employed (see Appendix A) and that a higher release was observed in test 3 over similar conditions but after all of the mechanical handling of the fuel segment.

\subsubsection{HBU 539B3 Rod Segment Test Results}

Table 4.7 through Table 4.9 list the results of the three rod segment tests for HBU 539B3. The estimated mass releases given in the tables are based on an assumed fuel density of $10 \mathrm{~g} / \mathrm{cc}$ and an OPC 
detection efficiency of 0.065 (see Section 6.3 .8 for details). Repeat tests were conducted in the order in which they are numbered and employ the same fuel-rod segment. As noted in the introduction to Section 4, tests 1 and 3 use a "plug up" orientation, whereas test 2 uses a "plug down" orientation. However, it must be noted that this sample was tested first using a cascade impactor and subsequently with the OPC.

As with the ATM-106 segment results, HBU 539B3 release results vary between repeat tests. The fuel released in the first test, $45 \mu \mathrm{g}$, is much lower than that released in the second and third tests, 225 and $213 \mu \mathrm{g}$, respectively. On the other hand, release results for the second and third tests compare well. Both show the same range of release over the 0 to 400 SCCM and 400 SCCM with mechanical action regions.

Table 4.7. Mass and Fractional Release for HBU 539B3 Rod Segment Test 1 of 3

\begin{tabular}{|l|c|c||}
\hline Region & $\begin{array}{c}\text { Estimated Mass } \\
\text { Release }[\mu \mathrm{g}]\end{array}$ & Release Fraction \\
\hline Release over 0 to 400 SCCM & 5 & $1.0 \times 10^{-7}$ \\
\hline Release at 400 SCCM, with mechanical action & 40 & $8.1 \times 10^{-7}$ \\
\hline Total & 45 & $9.2 \times 10^{-7}$ \\
\hline
\end{tabular}

Table 4.8. Mass and Fractional Release for HBU 539B3 Rod Segment Test 2 of 3

\begin{tabular}{|l|c|c||}
\hline Region & $\begin{array}{c}\text { Estimated Mass } \\
\text { Release }[\mu \mathrm{g}]\end{array}$ & Release Fraction \\
\hline Release over 0 to 400 SCCM & 81 & $1.7 \times 10^{-6}$ \\
\hline Release at 400 SCCM, with mechanical action & 144 & $2.9 \times 10^{-6}$ \\
\hline Total & 225 & $4.6 \times 10^{-6}$ \\
\hline
\end{tabular}

Table 4.9. Mass and Fractional Release for HBU 539B3 Rod Segment Test 3 of 3

\begin{tabular}{|l|c|c||}
\hline Region & $\begin{array}{c}\text { Estimated Mass } \\
\text { Release }[\mu \mathrm{g}]\end{array}$ & Release Fraction \\
\hline Release over 0 to 400 SCCM & 63 & $1.3 \times 10^{-6}$ \\
\hline Release at 400 SCCM, with mechanical action & 150 & $3.0 \times 10^{-6}$ \\
\hline Total & 213 & $4.3 \times 10^{-6}$ \\
\hline
\end{tabular}

Because tests 1 and 3 use the same "plug down" orientation, the increased release observed in test 3 suggests an opening of the fractures or increased the relocation of the fuel as a result of the mechanical actions applied in the first and second tests. On the other hand, the similarity in release between tests 2 and 3 suggests that orientation does not influence the fractional release for HBU 539B3. This latter observation may result from uniformity of the fractures throughout the rod segment. This is further supported by images of the HBU 539B3 rod segment in Figure 4.1c and Figure 4.1d where the exposed fuel on both ends appears relatively free of large cracks and void areas. 
Unlike the ATM 106 segment, the HBU 539B3 segment was difficult to flow gas through. Flow rates above 400 SCCM were not obtainable without exceeding the limiting pressure of the gas supply system (130 psig). The high gas pressure needed to obtain even modest flows is indicative of a closed fuel-clad gap and very tight fractures. This is also consistent with the lower release rates for this fuel: the rates observed here are roughly an order of magnitude lower than those observed in ATM 106 testing (where flow was not pressure limited).

As of the writing of this report, the cascade impactor had not yet been disassembled so that each individual filter could be counted. However, the entire impactor was placed on a gamma detector, and a preliminary gamma energy analysis was performed. There are large uncertainties because the geometry did not match a calibrated geometry for the system. Still, the strong gamma rays emitted by ${ }^{137} \mathrm{Cs}$ allowed the sample to be examined, and an estimated ${ }^{137} \mathrm{Cs}$ inventory of $1.10 \mu \mathrm{Ci}$ was found. Using the SCALE code data discussed in Section 2.3 and assuming a burnup of $60 \mathrm{GWd} / \mathrm{MTU}$ and a decay time of 15 years, this fuel is expected to have approximately $110 \mu \mathrm{Ci} \mathrm{mg}^{-1}$. Thus, the cascade impactor contains approximately $0.01 \mathrm{mg}(10 \mu \mathrm{g})$ of fuel, which is only a factor of 4 smaller than the release in Test 1 with the OPC using the efficiency of 0.065 , providing validity for the use of this global efficiency.

\subsubsection{HBU 539D5 Rod Segment Test Results}

Table 4.10 lists the results of the single rod-segment test for HBU 539D5 that was performed using the OPC. It should be noted that a test was first performed on this segment using a cascade impactor. The estimated mass releases given in the table are based on an assumed fuel density of $10 \mathrm{~g} / \mathrm{cc}$ and an OPC detection efficiency of 0.065 (see Section 6.3 .8 for details).

Table 4.10. Mass and Fractional Release for the HBU 539D5 Rod Segment Test

\begin{tabular}{|l|c|c||}
\hline Region & $\begin{array}{c}\text { Estimated Mass } \\
\text { Release }[\mu \mathrm{g}]\end{array}$ & Release Fraction \\
\hline Release over 0 to 500 SCCM & 148 & $3.1 \times 10^{-6}$ \\
\hline Release at 500 SCCM, with mechanical action & 79 & $1.7 \times 10^{-6}$ \\
\hline Total & 227 & $4.8 \times 10^{-6}$ \\
\hline
\end{tabular}

Like the HBU 539B3 segment tests, gas flows in HBU 539D5 were limited by the supply pressure of 130 psig. Here, the maximum achievable gas flow through the 539D5 segment was approximately $500 \mathrm{SCCM}$. As before, this suggests that the fuel-clad gap in this segment is closed, and the fractures are tight. Release rates from the first test are comparable to those observed in repeat tests of 539B3.

However, because only one test using the OPC was run for this fuel, the potential for increased release as a result of fuel microstructure alteration cannot be evaluated. It is interesting to note that the release over 0 to $500 \mathrm{SCCM}$ is greater than that observed at $500 \mathrm{SCCM}$ with mechanical action. This could indicate a clearing of easily fragmented material from the porous structure at early flow rates. It also could be a time effect, as the total testing time for the 0 to 500 -SCCM region ( $\sim 6000$ seconds) is much longer than that for the 500 SCCM with a mechanical action region ( $\sim 1500$ seconds). However, the latter conclusion is countered by the fact that each release event is allowed to continue until the counts return to background levels. 


\subsubsection{HBU 539F5 Rod Segment Test Results}

Table 4.11 and Table 4.12 list the results of the two rod segment tests for HBU 539F5. The estimated mass releases given in the tables are based on an assumed fuel density of $10 \mathrm{~g} / \mathrm{cc}$ and an OPC detection efficiency of 0.065 (see Section 6.3.8 for details).

Table 4.11. Mass and Fractional Release for HBU 539F5 Rod Segment Test 1 of 2

\begin{tabular}{|l|c|c||}
\hline Region & $\begin{array}{c}\text { Estimated Mass } \\
\text { Release }[\boldsymbol{\mu g}]\end{array}$ & Release Fraction \\
\hline Release over 0 to 500 SCCM & 68 & $1.4 \times 10^{-6}$ \\
\hline Release at 500 SCCM, with mechanical action & 257 & $5.2 \times 10^{-6}$ \\
\hline Release over 500 to 1000 SCCM & 0 & 0 \\
\hline Release over 1000 to 3000 SCCM & 54 & $1.1 \times 10^{-6}$ \\
\hline Release at 3000 SCCM and above, with mechanical action & 419 & $8.5 \times 10^{-6}$ \\
\hline Total & 798 & $1.6 \times 10^{-5}$ \\
\hline
\end{tabular}

Table 4.12. Mass and Fractional Release for HBU 539F5 Rod Segment Test 2 of 2

\begin{tabular}{|l|c|c||}
\hline Region & $\begin{array}{c}\text { Estimated Mass } \\
\text { Release }[\boldsymbol{\mu g}]\end{array}$ & Release Fraction \\
\hline Release over 0 to 500 SCCM & 169 & $3.4 \times 10^{-6}$ \\
\hline Release at 500 SCCM and beyond, with mechanical action & 225 & $4.6 \times 10^{-6}$ \\
\hline Total & 394 & $8.0 \times 10^{-6}$ \\
\hline
\end{tabular}

The two tests for HBU 539F5 not only show different release behaviors, but also different flow behaviors as well. In the first test, the flow rate was not pressure limited. That is, flow rates up to 3000 SCCM and beyond could be achieved without exceeding the maximum system pressure limit of $130 \mathrm{psig}$. In the second test, flow rates beyond 500 SCCM could not be achieved without exceeding a supply pressure of $130 \mathrm{psig}$.

One potential cause of the difference in achievable gas flows could be poor securing of the sample in the Swagelok ${ }^{\circledR}$. In this case, gas would flow around the outside of the fuel cladding, allowing gas flows that would be impossible if flow were restricted to the segment interior. However, poor securing of the Swagelok ${ }^{\circledR}$ fitting seems unlikely as significant particle entrainment (relative to other HBU tests) was observed during testing, which indicates significant flow through the interior. A more likely cause is that flow and mechanical action experienced during the first test collapsed gas flow paths. However, the 0- to 500-SCCM region in the second test shows a higher release relative to the first test, suggesting that the microstructure has been altered to allow higher particle releases (as in prior rod tests). On the other hand, compression of the cladding and fuel by the Swagelok ${ }^{\circledR}$ fitting could also locally collapse the flow pathways while leaving the altered microstructure downstream. Unfortunately, it is impossible to draw a conclusion as to the exact cause of the difference in achievable gas flows without information on how the internal structure of the rod changed from test-to-test and without information on the fuel orientation in the sample holder. 
Despite the difference in achievable flows, results from the first and second tests are similar for the 0 to $500-\mathrm{SCCM}$ region. The first test observed a $68-\mu \mathrm{g}$ release, whereas the second test observed a $169-\mu \mathrm{g}$ release. The increase in the second measurement release is consistent with previous tests and is believed to be caused by alterations in the microstructure favorable for particle release. Release results in the 500-SCCM region (with mechanical action) are comparable. Continued testing in the first HBU 539F5 measurement found a substantial drop in the particle release until there were flows above 3000 SCCM with mechanical action. This can be attributed to the mechanical action at 500 SCCM (which was not done in other tests). It is believed that tapping of the segment at 500 SCCM frees particles that would otherwise be entrained at higher gas flows.

\subsection{Rod-Segment Release-Fraction Results}

The preceding sections discuss particle release in terms of the mass of fuel released from the segment. This is useful only for comparing release under different gas-flow and mechanical-action regions within the same test rod. Release fractions (also reported in Table 4.4 to Table 4.12) provide a useful means of comparing results of different fuel segments. For the various fuel segments tests, the maximal total release results are

- $7.9 \times 10^{-5}$ for ATM 106

- $4.6 \times 10^{-6}$ for HBU $539 \mathrm{~B} 3$

- $4.8 \times 10^{-6}$ for HBU 539D5

- $1.6 \times 10^{-5}$ for HBU $539 \mathrm{~F} 5$.

These results indicate that the total release is greatest for ATM 106. However, they include the effects of repeat testing and may not be indicative of breach from an unperturbed fuel rod. When only the first test of each measurement series is considered (i.e., test 1 but ignoring the cascade impactor runs performed previously for two of the samples), the maximal total release is

- $1.1 \times 10^{-5}$ for ATM 106

- $9.2 \times 10^{-7}$ for HBU $539 \mathrm{~B} 3$

- $4.8 \times 10^{-6}$ for HBU 539D5

- $1.6 \times 10^{-5}$ for HBU 539 F5.

Within the uncertainties of the experimental system and analytic techniques, there is no difference between the release from ATM-106 and HBU 539F5 for these first tests. The other two HBU fuels exhibit smaller releases. The tests have shown, however, that high burnup fuel that contains HBS does not have any different release characteristics, either fractional release or PSD (see Appendix C), than other fuels other than the potential for smaller release because of restricted flow paths.

Accident release scenarios must also consider the flow region over which release occurs. Clearly, the release indicated by the total maximal release fraction does not occur all at once. For a given breach event, one of the flow regions given in Table 4.4 to Table 4.12 may be a more suitable indication of release. When the fuel structure is unaltered, and neglecting fuel densification, swelling, or clad creep, the nominal fuel pellet diameter and cladding inner diameters for the ATM-106 fuel (Guenther et al. 1988b, Figure 4.2) are 0.9639 and $0.9860 \mathrm{~cm}$, respectively. This yields a gap size of $0.221 \mathrm{~mm}$ and a 
cross-section area of $0.0338 \mathrm{~cm}^{2}$. Based on this information, the maximum gas velocity falls between the ranges shown in Table 4.13. It should be noted that the gas flow conditions listed in Table 4.13 correspond to standard conditions $\left(1 \mathrm{~atm}\right.$ and $\left.25^{\circ} \mathrm{C}\right)$.

Because of the high pressures involved (300 to 3000 psig depending on the initial fill gas pressure and the amount of fission gas released from the fuel pellet), gas flows in an actual rod depressurization event are typically sonic and are of very short duration. Scoping calculations of the depressurization event with helium at $3000 \mathrm{psig}$ as the rod fill gas found gas flows greater than $300 \mathrm{~m} / \mathrm{s}$ at standard conditions and depressurization times less than 1 second. Based on these scoping calculations alone, even the flows from 3000 to 9000 SCCM do not approach potential flows during pressure release of 3000 psig. Because rod depressurization supersedes (and as such, encompasses) all test conditions, no single flow regime provides a best estimate of actual release. For the purposes of release analysis herein, the total release will then be taken as the metric for fuel release during depressurization.

Table 4.13. Estimated Gas-Flow Velocity Inside Porous Interior of Fuel Rod Segments

\begin{tabular}{|l|c|}
\hline Region & Estimated Gas Flow Velocity [m/s] \\
\hline 0 to 500 SCCM & 0 to 2.47 \\
\hline 500 to 1000 SCCM & 2.47 to 4.93 \\
\hline 1000 to 3000 SCCM & 4.93 to 14.8 \\
\hline 3000 to 9000 SCCM & 14.8 to 44.38 \\
\hline
\end{tabular}

The release-fraction results presented in this section are best estimates of fuel release based on the mass detected at the OPC and on a conservative estimate of the OPC counting efficiency. The counting efficiency was determined for silica powder, and as such, may not be valid for spent-fuel particles because of differences in the material density, interaction potential, and charging behavior. Indeed, the influence of these differences will become apparent when the release results of spent-fuel powders are discussed in Section 5. In these results, the release estimated using silica detection efficiencies appears to underestimate release by 1 to 2 orders of magnitude. The consequence of this underestimation for the segment tests could mean that actual rod release fractions could be as high as $0.1 \%$. However, the preliminary results for the one cascade impactor test that was performed first suggest that the efficiency and methodology used for calculating release from the rod segments are valid; especially because the cascade impactor test did not suffer from the problem of excess air being forced out the balance air ports as occurred at high flow rates with the OPC because it can only draw in 1000 SCCM compared to the 9000 SCCM for the cascade impactor. 


\subsection{Execution of Oxidized Fuel Powder Tests}

Experimental studies for oxidized fuel powder tests employed the SAL FIATA setup described in the preceding sections. Four oxidized CSNF powders were tested:

- $109-\mathrm{A} 450$

- 109-A500

- 105-LOW

- 105-HIGH

Each of these consisted of fuels that had been oxidized previously in the TGA systems discussed in Section 2.3. The 109A fuels came from a segment adjacent to the HBU 539F5 segment discussed in Section 4.2 .4 and were oxidized at $450^{\circ} \mathrm{C}$ and $500^{\circ} \mathrm{C}$, respectively. The 105-LOW samples are a BWR fuel characterized in Guenther et al. (1991b) but came from the low burnup ( $\sim 15$ to $20 \mathrm{GWd} / \mathrm{MTU}$ ) end of the rod and were oxidized at $305^{\circ} \mathrm{C}$. The 105 -HIGH samples came from the higher burnup section $(\sim 28 \mathrm{GWd} / \mathrm{MTU})$ of the same rod and were also oxidized at $305^{\circ} \mathrm{C}$. All oxidized powder tests were carried out using optical particle counters. No measurements were made using cascade impactors.

Approximately 1 to $50 \mathrm{mg}$ of oxidized powders were transferred into pre-weighed sample boats. The exact powder mass added was then determined by mass difference (see Table 5.1). The sample boats with powder were then secured in the sample holder. The sample enclosure was then assembled and connected to the particle collection systems using a clean, 3-foot section of conductive tubing. As in the fuel segment tests, the length of tubing was maximized to reduce false OPC counts resulting from sample radioactivity.

Table 5.1. Mass of Oxidized Fuel Used in Powder Tests

\begin{tabular}{|l|c|}
\hline Sample & Powder Mass [mg] \\
\hline $109-A 450$ & 0.4 \\
\hline $109-A 500$ & 8.7 \\
\hline $105-$ LOW & 51.1 \\
\hline $105-$ HIGH & 50.3 \\
\hline
\end{tabular}

Once the sample holder was assembled, the OPC was powered on. A zero check was performed to verify that the system was sealed and that all balance air drawn into the system was being drawn through the HEPA filters. If the zero check was successful, the gas supply bottle was opened and regulated to a pressure above that necessary to overcome the 10-psig check valve. Valves V1 and V3 were opened and flows ranging from 0 to 200 SCCM were tested using flow controller FC1. At 200 SCCM, the flow control was given to controller FC2 by closing valve V1 and opening valve V2. Although different techniques were used to try to minimize any effects, the flow-controller exchange often led to a 1- to 2 -second spike in flow rate. This temporary spike, as high as 630 SCCM for 105-LOW, resulted in a significant release during each of the four tests. Gas flow rates from 200 SCCM to 9 SLM were tested using FC2. In some cases, the regulated gas pressure had to be increased to achieve the desired flow rate. While the $0.5-\mu \mathrm{m}$ porous frit at the base of the sample boat offered some resistance to flow, flow rates up 
to 9 SLM were achieved in all powder tests. At 3 SLM and beyond, the powders were subjected to tapping and pressure pulses (see previous section) to facilitate particle release.

The time of each flow-rate increase and/or mechanical action was recorded so that each observed particle release could be correlated to a change in conditions. Release events were monitored in real time through a computer connection to the particle analysis system. Particle counts were measured and recorded at a frequency of $1 \mathrm{~Hz}$. Typically, set points were held until the particle release caused by the last action returned to background levels.

At the end of each test, the FIATA was disassembled. Any visible powder fragments that had settled to the floor of the test apparatus enclosure were collected and added back to the sample boat. The actual powder release was determined by mass difference.

\subsection{Overall Results of CSNF Oxidized Powder Tests}

Summary results of the CSNF oxidized power tests are presented in Table 5.2 to Table 5.4. Table 5.2 gives a rough outline of the flow-rate ranges and mechanical actions each powder was subjected to during testing. Table 5.3 shows the characteristics of fuel release as detected by the OPC. It includes the raw particle counts, an estimate of the volume of spent fuel released, and the PSD in terms of the $10^{\text {th }}, 50^{\text {th }}$, and $90^{\text {th }}$ diameter percentiles for all events and mechanical actions performed on the listed powder sample.

Table 5.2. Summary of CSNF Oxidized Powder Testing Parameters

\begin{tabular}{|l|c|c|c|c|}
\hline \multicolumn{1}{|c|}{ System } & $\begin{array}{c}\text { Duration } \\
{[\mathbf{s}]}\end{array}$ & $\begin{array}{c}\text { Flow Rate Range } \\
{[\text { [SLM] }}\end{array}$ & Pressure Pulses & $\begin{array}{c}\text { Tapping/Shaking } \\
\text { Operations }\end{array}$ \\
\hline $109-\mathrm{A} 450$ & 475 & $0.01-9.0$ & 6 & 0 \\
\hline $109-\mathrm{A} 500$ & 2816 & $0.01-9.0$ & 3 & 3 \\
\hline 105 -LOW & 1032 & $0.01-9.0$ & 2 & 8 \\
\hline 105 -HIGH & 1434 & $0.01-9.0$ & 2 & 10 \\
\hline
\end{tabular}

Table 5.3. Summary of CNSF Oxidized Powder Test Release Results

\begin{tabular}{||l|c|c|c|c|c||}
\hline \multicolumn{1}{|c|}{ System } & $\begin{array}{c}\text { Counts } \\
{[\text { particles] }}\end{array}$ & $\begin{array}{c}\text { Volume } \\
\text { Released }\left[\mathbf{c m}^{3}\right]\end{array}$ & $\begin{array}{c}\mathbf{d 1 0} \\
{[\boldsymbol{\mu m}]}\end{array}$ & $\begin{array}{c}\mathbf{d 5 0} \\
{[\boldsymbol{\mu m}]}\end{array}$ & $\begin{array}{c}\mathbf{d 9 0} \\
{[\boldsymbol{\mu m}]}\end{array}$ \\
\hline $109-\mathrm{A} 450$ & $4.2 \times 10^{2}$ & $4.4 \times 10^{-8}$ & 3.6 & 12.2 & 16.1 \\
\hline $109-\mathrm{A} 500$ & $3.7 \times 10^{4}$ & $6.0 \times 10^{-7}$ & 1.8 & 5.4 & 15.7 \\
\hline $105-\mathrm{LOW}$ & $7.2 \times 10^{4}$ & $1.3 \times 10^{-6}$ & 1.8 & 5.9 & 15.0 \\
\hline $105-\mathrm{HIGH}$ & $7.0 \times 10^{3}$ & $5.7 \times 10^{-7}$ & 2.9 & 10.0 & 17.5 \\
\hline
\end{tabular}

It should be noted that because of the small sample mass associated with test 109-A450 (0.4 mg), the results may be atypical and unreliable. In addition, the low powder mass in both 109-A450 and 109-A500 samples makes comparison against powder-release results for 105-LOW and 105-HIGH tenuous. 
Table 5.4 shows the mass release as determined by the difference between mass of powder added to the sample boat and mass of powder remaining after the test. In all tests, a loss of powder measurable by a four-place balance was observed. The release fraction as indicated by mass difference was high, ranging from $9 \%$ up to $80 \%$. It should be noted that the $80 \%$ release occurred for the $109-\mathrm{A} 450$. As such, this release fraction is somewhat unreliable because of the low initial mass of powder ( $0.4 \mathrm{mg})$. If a balance uncertainty of $\pm 0.1 \mathrm{mg}$ is assumed, the uncertainty introduced by the balance alone in the $80 \%$ release fraction result is $\pm 30 \%$. Relative to the rod segment tests, the release fractions measured for oxidized powders are at least five orders of magnitude larger. This result is expected, as the fuel particles and particle aggregates are not confined by the microstructure of the fuel or by the cladding.

Table 5.4. Mass and Fractional Release for the CSNF Powder Test

\begin{tabular}{||l|c|c||}
\hline System & $\begin{array}{c}\text { Estimated Mass } \\
\text { Release }[\mathbf{m g}]\end{array}$ & Release Fraction \\
\hline $109-\mathrm{A} 450$ & 0.3 & 0.75 \\
\hline $109-\mathrm{A} 500$ & 5.0 & 0.57 \\
\hline $105-\mathrm{LOW}$ & 14.2 & 0.278 \\
\hline $105-\mathrm{HIGH}$ & 4.5 & 0.0895 \\
\hline
\end{tabular}

The release results shown in Table 5.4 must be used with caution when evaluating a potential release of oxidized fuel powder. Specifically, these results encompass the total release caused by the full range of flow and mechanical conditions that were examined during testing. Specifically, the volumetric flow rate in all tests went as high as $9000 \mathrm{SCCM}$, which corresponds to a linear velocity greater than $2 \mathrm{~m} / \mathrm{s}$. This velocity is more than one order of magnitude higher than that expected inside a failed container (BSC 2007a). This, combined with the fact that the powder rests inside the gas flow field, yields high release results relative to other oxidized powder studies (such as that by Mishima [1969]). It should be noted that in a scoping study performed by Mishima (as described in the 1969 report) in which oxidized plutonium oxide powders were allowed to directly interact with a high-velocity gas stream, similar release results to those reported in Table 5.4 were observed. In the following sections, the release will be examined as a function of flow and mechanical action.

\subsection{Individual Results of CSNF Oxidized Powder Tests}

As with the rod tests, the overall release results given in Section 5.1 represent the sum of particle release as a result of each flow set-point change and mechanical action. While this information is useful in gauging the physical properties of the fuel, such as the propensity to release particles relative to one another and the average size distribution of particles reaching the detector, it provides little information on how flow conditions affect particle release.

In the following subsections, particle release as a function of flow conditions and mechanical action will be provided. Powder tests involved anywhere between 50 to 80 individual "events." For brevity, the results presented in this section will examine release over four flow-rate/agitation regimes:

- between 0 to 500 SCCM with no mechanical action

- between 500 to 1000 SCCM with no mechanical action 
- between 1000 to 3000 SCCM with no mechanical action

- 3000 SCCM and above with mechanical action.

An event-by-event breakdown of particle release is provided in Appendix B, and PSDs are shown in Appendix D. Note that because of rounding, the totals in some of the tables will appear to be incorrect. For consistency, all values are taken from the associated spreadsheets and then rounded to the appropriate number of significant figures in this document.

\subsubsection{9-A450 Powder Test Results}

Table 5.5 lists the results of the single powder test for 109-A450. The estimated mass releases given in the table are based on an assumed fuel density of $8.35 \mathrm{~g} / \mathrm{cc}$ (i.e., the theoretical density of $\mathrm{U}_{3} \mathrm{O}_{8}$ ) and an OPC detection efficiency of 0.065 (see Section 6.3 .8 for details).

Table 5.5. Mass and Fractional Release for the 109-A450 Powder Test

\begin{tabular}{|l|c|c||}
\hline Region & $\begin{array}{c}\text { Estimated Mass } \\
\text { Release }[\boldsymbol{\mu g}]\end{array}$ & Release Fraction \\
\hline Release over 0 to 500 SCCM & 5 & $1.3 \times 10^{-2}$ \\
\hline Release over 500 to 1000 SCCM & 0 & 0 \\
\hline Release over 1000 to 3000 SCCM & 0 & 0 \\
\hline Release at 3000 SCCM and above, with mechanical action & 1 & $2.5 \times 10^{-3}$ \\
\hline Total & 6 & $1.5 \times 10^{-2}$ \\
\hline
\end{tabular}

Based on the results of the OPC efficiency analysis, 109-A450 oxidized fuel powder shows a total estimated mass release of $6 \mu \mathrm{g}$. This release is two orders of magnitude lower than measured directly by mass difference ( $\sim 0.3 \mathrm{mg}$, see Table 5.4$)$. The difference in actual and estimated release likely results from material differences between the spent-fuel particles and the silica powder used to establish the efficiency. Specifically, differences in particle morphology and density as well as differences in interaction or sticking potential (such as particle charging) will lead to differences in the propensity of the dispersed phase to settle and/or adhere to surfaces.

Despite the difference in total mass detection, it is assumed that mass derived from OPC detection provides an indication of the relative release between flow regions. As such, the results in Table 5.5 suggest that the majority of release occurs over the 0- to 500-SCCM range. Based on the information in Appendix B, the release over the first region appears to be driven by the flow controller exchange at 200 SCCM when the flow rate exhibited a temporary spike to 300 SCCM. Approximately $80 \%$ of the total release in this test was associated with the flow increase during the flow-controller exchange. This corresponds to $100 \%$ of the release in the flow range most applicable to the scenarios of interest ( 0 to $500 \mathrm{SCCM})$. In addition, the small $1-\mu \mathrm{g}$ release appears to be driven by mechanical action rather than flow. Both release events suggest a powder that is difficult to disperse, as sudden flow alterations and mechanical action are required to promote the travel of fuel particles to the OPC. The larger release of $5 \mu \mathrm{g}$ suggests that the flow spike during the 200-SCCM controller exchange dispersed all of the loose powder, leaving behind those particles strongly adhered to the walls of the boat and test apparatus. The 
latter were subsequently disturbed by mechanical action, as given by the $1-\mu \mathrm{g}$ release in the $3000 \mathrm{SCCM}$ and above region.

The total release fraction of powder 109-A450 estimated by this test is $1.5 \times 10^{-2}$. Relative to the other oxidized powder tests, OPC results for 109-A450 showed the largest degree of fuel release. This observation is consistent with the release-fraction measurements based on the difference between starting and ending boat mass (see Table 5.4). This result should be viewed with some caution, as the low starting mass of material introduces a large uncertainty into the release fraction.

\subsubsection{9-A500 Powder Test Results}

Table 5.6 lists the results of the single powder test for 109-A500. The estimated mass releases given in the table are based on an assumed fuel density of $8.35 \mathrm{~g} / \mathrm{cc}$ and an OPC detection efficiency of 0.065 (see Section 6.3 .8 for details).

Table 5.6. Mass and Fractional Release for the 109-A500 Powder Test

\begin{tabular}{|l|c|c||}
\hline Region & $\begin{array}{c}\text { Estimated Mass } \\
\text { Release }[\boldsymbol{\mu g}]\end{array}$ & Release Fraction \\
\hline Release over 0 to 500 SCCM & 0.6 & $6.9 \times 10^{-5}$ \\
\hline Release over 500 to 1000 SCCM & 1.7 & $2.0 \times 10^{-4}$ \\
\hline Release over 1000 to 3000 SCCM & 11 & $1.3 \times 10^{-3}$ \\
\hline Release at 3000 SCCM and above, with mechanical action & 64 & $7.4 \times 10^{-3}$ \\
\hline Total & 77 & $8.9 \times 10^{-3}$ \\
\hline
\end{tabular}

For the total range of flow and mechanical conditions, the total mass release for powder 109-A500 was $77 \mu \mathrm{g}$. With the powder 109-A450, this mass release is approximately two orders of magnitude lower than that determined by differential mass measurements $(5.0 \mathrm{mg})$. It is postulated that differences in the sticking potential, holdup, particle characteristics (morphology and density) and settling of the oxidized spent-fuel powder relative the silica powder used to calibrate release caused the large difference between release estimated by OPC and that measured by mass difference. The release of powder 109A500 appears to increase with increasing gas volumetric flow rate and mechanical action. In relative terms, only minor release occurs at flow rates spanning 0 to 1000 SCCM. Approximately $90 \%$ of the release in the range of interest ( 0 to $500 \mathrm{SCCM}$ ) was associated with the flow spike during the flow controller exchange (see Appendix B), although the peak flow rate during the spike was not recorded.

Although increased fuel release is observed between 1000 to 3000 SCCM, the vast majority of release occurs at flow rates over 3000 SCCM. The total release fraction of powder 109-A450 estimated by this test is $8.9 \times 10^{-3}$. Of all the oxidized powder tests, this is the second largest release. The magnitude of relative release is again consistent with differential mass results in Table 5.4. Specifically, powder 109A500 shows the second largest release fraction based on both OPC and differential mass results. Relative to 109-A450, powder 109-A500 typically shows a higher mass release with the exception of the 0 - to 500-SCCM region. However, this appears to be an effect of having more fuel available for release, as the release fractions for A500 are roughly an order of magnitude lower than those for A450. 


\subsubsection{5-LOW Powder Test Results}

Table 5.7 lists the results of the single powder test for 105-LOW. The estimated mass releases given in the table are based on an assumed fuel density of $8.35 \mathrm{~g} / \mathrm{cc}$ and an OPC detection efficiency of 0.065 (see Section 6.3.8 for details).

Table 5.7. Mass and Fractional Release for the 105-LOW Powder Test

\begin{tabular}{|l|c|c||}
\hline Region & $\begin{array}{c}\text { Estimated Mass } \\
\text { Release }[\boldsymbol{\mu g}]\end{array}$ & Release Fraction \\
\hline Release over 0 to 500 SCCM & 120 & $2.3 \times 10^{-3}$ \\
\hline Release over 500 to 1000 SCCM & 0 & 0 \\
\hline Release over 1000 to 3000 SCCM & 26 & $5.1 \times 10^{-4}$ \\
\hline Release at 3000 SCCM and above, with mechanical action & 22 & $4.3 \times 10^{-4}$ \\
\hline Total & 169 & $3.3 \times 10^{-3}$ \\
\hline
\end{tabular}

The total mass release for powder $105-\mathrm{LOW}$ was $168 \mu \mathrm{g}$. As with previous tests, this mass release is again two orders of magnitude lower than that determined by differential mass measurements $(14.2 \mathrm{mg})$. The release of 105-LOW powder is influenced by the flow-controller exchange. Specifically, the $120 \mu \mathrm{g}$ release between 0 to $500 \mathrm{SCCM}$ is associated with the flow-controller exchange at 200 SCCM.

Transient, but significant, increases in gas flow typically occur during the flow-controller exchange because of the inability of the controller to maintain steady flow as valve 1 is shut and valve 2 is opened. For the 105-LOW powder, a flow-rate spike of 630 SCCM occurred during the exchange. This flow-rate spike, coupled with the fact that 105-LOW also had the most initial mass of powder in the boat ( $\sim 50.0 \mathrm{mg}$ ), probably led to the large particle release during the exchange. The release associated with the flow-rate spike is approximately $71 \%$ of the total release and is virtually $100 \%$ of the release at flows below 500 SCCM (see Appendix B). The large spike in gas flow as a result of the flow-controller exchange appears to "purge" most of the easily dispersible material from the sample boat, as no particle release occurs in the 500- to 1000-SCCM region. Higher flows and mechanical action appear to yield some particle release, but these do not match the release caused by the flow controller exchange.

The total release fraction of powder 105 -LOW estimated by this test is $3.3 \times 10^{-3}$. Of all the oxidized powder tests, this is the third largest release. The magnitude of relative release is again consistent with differential mass results in Table 5.4. Specifically, powder 105-LOW shows the third largest release fraction based on both OPC and differential mass results.

\subsubsection{5-HIGH Powder Test Results}

Table 5.8 lists the results of the single powder test for 105-HIGH. The estimated mass releases given in the table are based on an assumed fuel density of $8.35 \mathrm{~g} / \mathrm{cc}$ and an OPC detection efficiency of 0.065 (see Section 6.3.8 for details). 
Table 5.8. Mass and Fractional Release for the 105-HIGH Powder Test

\begin{tabular}{|l|c|c||}
\hline \hline Region & $\begin{array}{c}\text { Estimated Mass } \\
\text { Release }[\boldsymbol{\mu g}]\end{array}$ & Release Fraction \\
\hline Release over 0 to 500 SCCM & 9 & $1.8 \times 10^{-4}$ \\
\hline Release over 500 to 1000 SCCM & 0 & 0 \\
\hline Release over 1000 to 3000 SCCM & 4 & $8.0 \times 10^{-5}$ \\
\hline Release at 3000 SCCM and above, with mechanical action & 60 & $1.2 \times 10^{-3}$ \\
\hline Total & 73 & $1.5 \times 10^{-3}$ \\
\hline
\end{tabular}

The total mass release for powder $105-\mathrm{HIGH}$ was $73 \mu \mathrm{g}$. As with previous tests, this mass release is again two orders of magnitude lower than that determined by differential mass measurements (4.5 mg). The release of 105-HIGH powder over the 0- to 500-SCCM flow region appears to be influenced by the flow-controller exchange, as the 9- $\mu$ g release is higher than the mass release observed in the 500- to 1000 -SCCM and 1000- to 3000-SCCM flow regions ( 0 and $4 \mu \mathrm{g}$, respectively). Flows in excess of 3000 SCCM and mechanical action yield the strongest particle release of $60 \mu \mathrm{g}$. In contrast to the 105-LOW powder test, the 105-HIGH powder test showed relatively low particle releases over the 0 to 500-SCCM range. Although both 105-LOW and 105-HIGH powder tests started with approximately the same amount of oxidized powder $(\sim 50 \mathrm{mg})$, the flow controller exchange in the 105-HIGH test was not accompanied by the typical transient increase in gas flow that was the driving force for the large release in the 105-LOW test. The release during the controller transition accounts for $100 \%$ of the release in the 0 to 500 -SCCM range, but only about $12 \%$ of the total release when release due to mechanical action is included.

The total release fraction of powder 105 -HIGH estimated by this test is $1.4 \times 10^{-3}$. Of all the oxidized powder tests, this is the fourth largest release. The magnitude of relative release is again consistent with differential mass results in Table 5.4. Specifically, powder 105-HIGH shows the fourth largest release fraction based on both OPC and differential mass results.

\subsection{Oxidized Powder Release Fraction Results Discussion}

The mass release and release fractions for oxidized powders discussed in the preceding sections indicate that there is a potential for large release if the gas stream directly interacts with oxidized powder. This result is based on the release fractions shown in Table 5.4. Even if results for powder 109-A450 are discarded because of low sample mass, release fractions still range from 9 to $57 \%$ for the conditions tested. Such large releases are expected given the experimental conditions, as Mishima (1969) observed similar release in a scoping study where powder directly interacted with the air stream. In fact, the porous frit produces a well fluidized stream and maximizes the potential for release by overcoming factors such as sticking potentials. As such, application of such release fractions to accident scenarios within the surface facilities at Yucca Mountain should be performed with caution, especially since most fuel will be in a canister (e.g., cask, TAD, waste package) that will greatly limit the exposure of fuel to flowing air.

The difference in the actual release measured by mass difference and that estimated by OPC detection efficiency calculations indicates the degree to which material reaching the OPC depends on the material properties. This difference is important when evaluating potential release. As shown in Section 6, the lower bounding detection efficiency for silica used to estimate fuel release was $6.5 \%$. For the results in 
this section, comparison of boat-mass-loss measurement to estimated mass release by OPC consistently determines release estimates that are two orders of magnitude lower than expected. As such, the true detection efficiency for spent fuel may be as low as $\sim 0.1 \%$. Of the two methods, release fractions determined by mass difference are the most direct and reliable measurement of fuel transport. Specifically, the 9 to $57 \%$ release fractions are the most reliable measurements because they make no assumption as to the behavior of the fuel. In contrast, the results estimated from OPC detection make the implicit assumption that the fuel behaves like silica with regard to sticking and settling. This conclusion impacts the results of Section 4, as it indicates that fuel-release fractions from rods may actually be on the order of $1 \times 10^{-3}$ as opposed to the $1 \times 10^{-5}$ fraction measured by OPC detection.

The release fractions measured for oxidized powders depend on the flow region in which they were taken. Some part, if not the majority, of the release occurred as a result of flows greater than 3000 SCCM and mechanical agitation in all tests. The 9 to $57 \%$ release fractions discussed in the preceding paragraph encompass the entire range of test conditions herein, including both flow and mechanical action. Relative to this, test conditions observed in the experiments above may not be indicative of those that occur at the surface facilities. Estimates of the linear superficial gas-flow velocity acting on the spent-fuel powder in the flow region can be made based on the known range of gas flow rates tested and on the known 0.374-in. inner diameter of the sample boat. These are shown in Table 5.9. The flow regions identified for testing span gas-flow velocities from 0 up to $2.1 \mathrm{~m} / \mathrm{s}$. However, gas-flow velocities inside a breached canister will most likely result from natural convention and should have an upper bound of approximately $0.1 \mathrm{~m} / \mathrm{s}$ (BSC2007a). As such, test results in the 0 to 500-SCCM flow region are the most appropriate measures of fuel release and dispersion resulting from natural convection.

Table 5.9. Estimated Linear Gas Flow Velocity Inside Powder Sample Boat

\begin{tabular}{|l|c|}
\hline Region & Estimated Gas Flow Velocity [m/s] \\
\hline 0 to 500 SCCM & 0 to 0.12 \\
\hline 500 to 1000 SCCM & 0.12 to 0.24 \\
\hline 1000 to 3000 SCCM & 0.24 to 0.71 \\
\hline 3000 to 9000 SCCM & 0.71 to 2.1 \\
\hline
\end{tabular}

Given the 0 - to 500-SCCM region of interest, the release fractions based on OPC detection and efficiency calculations are:

- $\quad 1.3 \times 10^{-2}$ for $109-\mathrm{A} 450(\sim 5 \mu \mathrm{g}$ released out of $0.4 \mathrm{mg}$ available $)$

- $6.9 \times 10^{-5}$ for $109-\mathrm{A} 500(\sim 0.6 \mu \mathrm{g}$ released out of $8.7 \mathrm{mg}$ available $)$

- $2.3 \times 10^{-3}$ for $105-\mathrm{LOW}(\sim 120 \mu \mathrm{g}$ released out of $51.1 \mathrm{mg}$ available)

- $1.8 \times 10^{-4}$ for $105-\mathrm{HIGH}(\sim 9 \mu \mathrm{g}$ released out of $50.3 \mathrm{mg}$ available).

In order of most easily to least easily dispersible at low flows, the powders are 109-A450, 105-LOW, 109-A500, and 105-HIGH. It should be noted that these release fractions are subject to the uncertainty associated with the OPC detection efficiency and, as such, may be one to two orders of magnitude larger than indicated by the OPC test results. 
Because the total release fractions for OPC and mass difference analyses predict the same order of release for the powders, an estimate of the "actual" release fraction for 0 to 500 SCCM can be made by multiplying the 0 - to 500-SCCM OPC release fractions by the ratio of total differential mass to total OPC detected mass. For example, release results for powder 109-A450 are $0.3 \mathrm{mg}$ by mass difference (from Table 5.4) and $6 \mu \mathrm{g}$ by OPC detection analysis (from Table 5.5). An estimate of the "actual" release is then:

$$
\left(\frac{0.3 \mathrm{mg}}{6 \mu \mathrm{g}}\right)\left(\frac{1000 \mu \mathrm{g}}{1 \mathrm{mg}}\right)\left(1.3 \times 10^{-2}\right)=0.65
$$

Thus, the "corrected" release fractions for oxidized spent-fuel powders within the flow regime of interest may be as follows:

- $\quad 0.65$ for $109-\mathrm{A} 450$

- 0.0045 for $109-\mathrm{A} 500$

- 0.19 for $105-\mathrm{LOW}$

- 0.011 for $105-\mathrm{HIGH}$.

All in all, these results suggest that spent-fuel powders are highly dispersible, even at relatively low linear gas velocities. However, the 0.19 and 0.65 release fractions for $109-\mathrm{A} 450$ and 105 -LOW could be atypical. The 109-A450 powder results are prone to high uncertainty because of the low starting mass of powder, and the 105-HIGH powder results showed atypical release over the 0 to 500-SCCM region because the flow actually reached 630 SCCM during the flow controller exchange. Release fractions for 109-A500 and 105-HIGH powders are on the order of $5 \times 10^{-3}$ to $1.0 \times 10^{-2}$. Although both are still large relative to those reported in Mishima's 1969 study $\left(5 \times 10^{-6}\right.$ to $\left.3 \times 10^{-3}\right)$, Mishima's results correspond to air flows above the powder rather than through the powder as in the present study. As such, large release fractions should be expected using the FIATA, as the release conditions simulate Stokes suspension of particles (discussed in Section 7 of this report). Even then, virtually no release was detected in this flow range except under the transient condition of the flow-controller exchange, whether or not there was a corresponding detectable flow increase.

\subsection{Particle Size of Oxidized Powders}

Although the discussion of oxidized powder studies has focused primarily on the degree of release, the main objective was to obtain a measurement of the size fractionation of particles. The PSD of the oxidized powders was derived directly from OPC results as discussed in Section 3.8 of this report. Table 5.10 presents the results of the size analysis. Specifically, it lists $10^{\text {th }}, 50^{\text {th }}$, and $90^{\text {th }}$ percentiles based on the particle count fractionation of the sum of all non-background release events. An event-byevent breakdown of PSD is provided in Appendix D to this report. 
Table 5.10. Particle-Size Distribution for the Sum of All Non-Background Release Events Observed in Oxidized Powder Tests

\begin{tabular}{||l|c|c|c||}
\hline Powder & $\mathbf{d}(\mathbf{1 0})[\boldsymbol{\mu m}]$ & $\mathbf{d}(\mathbf{5 0})[\boldsymbol{\mu m}]$ & $\mathbf{d}(\mathbf{9 0})[\boldsymbol{\mu m}]$ \\
\hline $109-\mathrm{A} 450$ & 3.63 & 12.2 & 16.1 \\
\hline $109-\mathrm{A} 500$ & 1.83 & 5.40 & 15.7 \\
\hline 105-LOW & 1.84 & 5.94 & 15.0 \\
\hline 105-HIGH & 2.92 & 9.97 & 17.5 \\
\hline
\end{tabular}

The particle-size results in Table 5.10 indicate that, for all powders, $10 \%$ of the particles by mass fall below 2 to $4 \mu \mathrm{m}$. Mean particle diameters as given by the $\mathrm{d}(50)$ fall between 5 and $12 \mu \mathrm{m}$. The upper range of particle size falls close to 15 to $20 \mu \mathrm{m}$. However, because of the limited-size measurement range of the particle counters, the upper end of the PSD could not be probed. For all tests, the sum of nonbackground release events showed particles larger than $20 \mu \mathrm{m}$ present. However, because of the inability of the Met-One OPC to size in this range, the actual percentage of particles or particle aggregates in this size range could not be determined.

From Table 5.10, it is clear that the 105-LOW fuel had a smaller particle size than did the 105-HIGH fuel. This is to be expected since they both had the same pre-irradiation grain size $(\sim 11 \mu \mathrm{m}$ per Table 4.11 in Guenther et al. 1991b) but the fuel in the hotter, higher burnup section of the fuel experienced grain growth, so the post-irradiation grain size was larger ( $\sim 14$ to $15 \mu \mathrm{m})$. Thus, the 105-HIGH sample should have approximately the same post-irradiation grain size as the 109-A samples (Vaidyanathan et al. 1997). Table 5.10 shows that $105-$ HIGH and 109-A450 had similar post-oxidation particle sizes. As discussed in Section 2.2.2, the post-oxidation grain size is a function of not only the starting grain size, but also the temperature at which oxidation occurs. It appears that intragranular cracking (see Figure 2.4) is more extensive at higher temperatures and may lead to smaller particulates, as demonstrated by the smaller particle sizes for 109-A500 compared to 109-A450. These differences in morphology and particle size may influence properties such as sticking and release, although no direct correlation between the PSDs in Table 5.10 and the release fractions discussed in Section 5.3 is readily apparent. Still, there is no evidence that the HBS contributes to additional release or markedly different PSDs. 


\subsection{Calibration of Total Versus Detected Release Using Silica Powders}

During testing of fuel-rod segments and oxidized fuel powders, only a fraction of the free particles and particle aggregates generated actually make it to the particle collection/analysis system. Loss occurs as a result of material sticking to the internal surfaces of the test apparatus, large material fragments settling, and/or sample boat effluent splitting.

Because the primary fuel particles are small $(\sim 0.5$ to $20 \mu \mathrm{m})$ and potentially charged as a result of radioactivity, there is a significant probability that some of the released material will stick to the internal surfaces of the test apparatus as a result of van der Waals and/or Coulombic attraction. Surfaces available for particle sticking include the enclosure body and cap and the internal diameter of the conductive tubing between the enclosure and particle-collection system.

The balance air to the system, which forms a sheath around the sample boat, may not be sufficient to suspend all particles exiting the sample boat, including large particles and particle aggregates. As a result, material released can settle to the base of the sample chamber after exiting the sample boat. Settling is compounded by the sudden enlargement and resulting reduction in suspending gas velocity that occurs at the sample boat and by the decrease in balance air velocity as the suspending gas velocity increases.

The sample boat effluent splits when the gas-supply flow rate through the sample boat exceeds that drawn into the downstream particle collection and analysis system. It results from the fact that the particle collection systems are designed to sample the enclosure at a single flow rate: 1 SLM for OPCs and 9 SLM for cascade impactors. Because gas-supply flows are limited to 9 SLM, flow splitting only affects measurements employing the OPC. Specifically, for OPC measurements at flows greater than 1 SLM, the excess flow and any entrained particles are forced out of HEPA filter \#2 (see Figure 3.3) to maintain air balance. Assuming perfect mixing of the entrained particles in the enclosure, losses in OPC detection efficiency as a result of splitting can be estimated based on the ratio of sampling flow to total gas flow. For example, at 3 SLM, the counting efficiency is (1 SLM/3 SLM) 33\%.

The actual release of material can be expressed in terms of a counting efficiency, which is defined as the ratio of release mass/volume detected at the collection system to that actually released from the sample boat. Experimental determination of the counting efficiency is possible provided that both the mass released from the boat and that collected downstream can be tracked throughout the measurement. However, this is impractical in the hot-cell environment because of the time required to disassemble and reassemble the FIATA (approximately 1-hour)

To estimate the counting efficiency of the particle-collection systems used in fuel-in-air testing, tests using a standardized silica powder were performed on a non-radioactive mock-up of the FIATA system. The non-rad FIATA mock-up is equivalent to the SAL FIATA. Because the majority of tests on fuel-rod segments and oxidized powders used the OPC as the collection system and because of the difficulties associated with detecting small quantities of collected materials on the cascade impactor stages, the total versus detected release of particles was calibrated using only the OPC. 


\subsection{Execution of Silica Calibration Tests}

Experimental calibration of FIATA/OPC counting efficiency employed a non-radioactive mock-up of the SAL FIATA. The silica powder used for testing was a 1- to $10-\mu \mathrm{m}$ polydisperse silica particle size standard, PS-192, from Whitehouse Scientific, Ltd. (Waverton, Chester, CH3 7PB, United Kingdom). Whitehouse Scientific supplies these standards as 0.1-g dry-powder shots. The specific details of the powder are given in Table 6.1. All silica calibration tests were carried out using optical particle counters. No measurements were made using cascade impactors.

Table 6.1. Properties of the Particle Size Standard Used in Silica Calibration Testing. The material was obtained from Whitehouse Scientific. The manufacturer's listed PSD [i.e., the d(10), d(50), and $\mathrm{d}(90)]$ corresponds to a well-dispersed suspension of the material in water.

\begin{tabular}{||l|l|}
\hline Size Range: & $1-10 \mu \mathrm{m}$ \\
\hline Catalogue \#: & PS-192 \\
\hline $\mathrm{d}(10):$ & $2.88 \mu \mathrm{m}$ \\
\hline $\mathrm{d}(50):$ & $4.18 \mu \mathrm{m}$ \\
\hline $\mathrm{d}(90):$ & $6.23 \mu \mathrm{m}$ \\
\hline
\end{tabular}

For each calibration measurement, approximately 100 to $150 \mathrm{mg}$ of silica powder was transferred into a pre-weighed sample boat. The exact powder mass added was then determined by mass difference. The sample boat with powder was then secured in the sample holder. The sample enclosure was then assembled and connected to the particle collection systems using a clean, 3-foot section of conductive tubing.

Once the sample holder was assembled, the OPC was powered on. A zero check was performed to verify that the system was sealed and that all balance air drawn into the system was being drawn through the HEPA filters. Four tests were run to determine the counting efficiency over a range of conditions:

- Test 1: counting efficiencies over flow rates between 100 and 1000 SCCM without any mechanical action. For this test, the gas flow rate was increased at 100-SCCM intervals. Flow set points were held for 5 minutes while the particle release was continuously monitored by OPC. After each flow set point, the gas flow was stopped, the enclosure disassembled, and the sample boat weighed to determine powder loss by difference. All operations used flow controller FC2.

- Test 2: counting efficiencies over flow rates between 100 and 1000 SCCM with mechanical action. For this test, the gas flow rate was again increased at 100-SCCM intervals. Flow set points were held for 5 minutes while the particle release was continuously monitored by OPC. For the first 30 seconds of the flow set point, the enclosure housing was tapped with a wrench to assist particle release. The enclosure housing was disassembled after the 800- and 1000-SCCM set points to measure the release of powder from the boat. All operations used flow controller FC2.

- Test 3: counting efficiencies were determined for a flow set point and mechanical action conditions similar to those used in hot cell testing. The flow was ramped from 0 to 3 SLM, with a flow controller exchange at 200 SCCM. The enclosure housing was tapped at 3 SLM to assist release. 
The test apparatus was disassembled at 1 SLM and 3 SLM (before and after mechanical action) to determine powder release from the sample boat. For these tests, flow set points were held for at least 5 minutes. In some cases, the set point was held up to 15 minutes to allow particle counts to decay.

- Test 4: uses a modified mock-up system. The sample enclosure is bypassed by making a direct connection between the sample boat and OPC. This eliminates the possibility of settling in the sample chamber. Balance air is provided by a plastic $3 / 8$ in. Y-junction. The total length of the connection between the boat and OPC is no longer than 3 inches. A schematic of the modified test setup is shown in Figure 6.1. Flow rates tested go from 100 to 1000 SCCM at 100-SCCM intervals without any mechanical action. Flow set points were held for 5 minutes while the particle release was continuously monitored by OPC. After each flow set point, the gas flow was stopped, the enclosure disassembled, and the sample boat weighed to determine powder loss by difference. All operations used flow controller FC2.

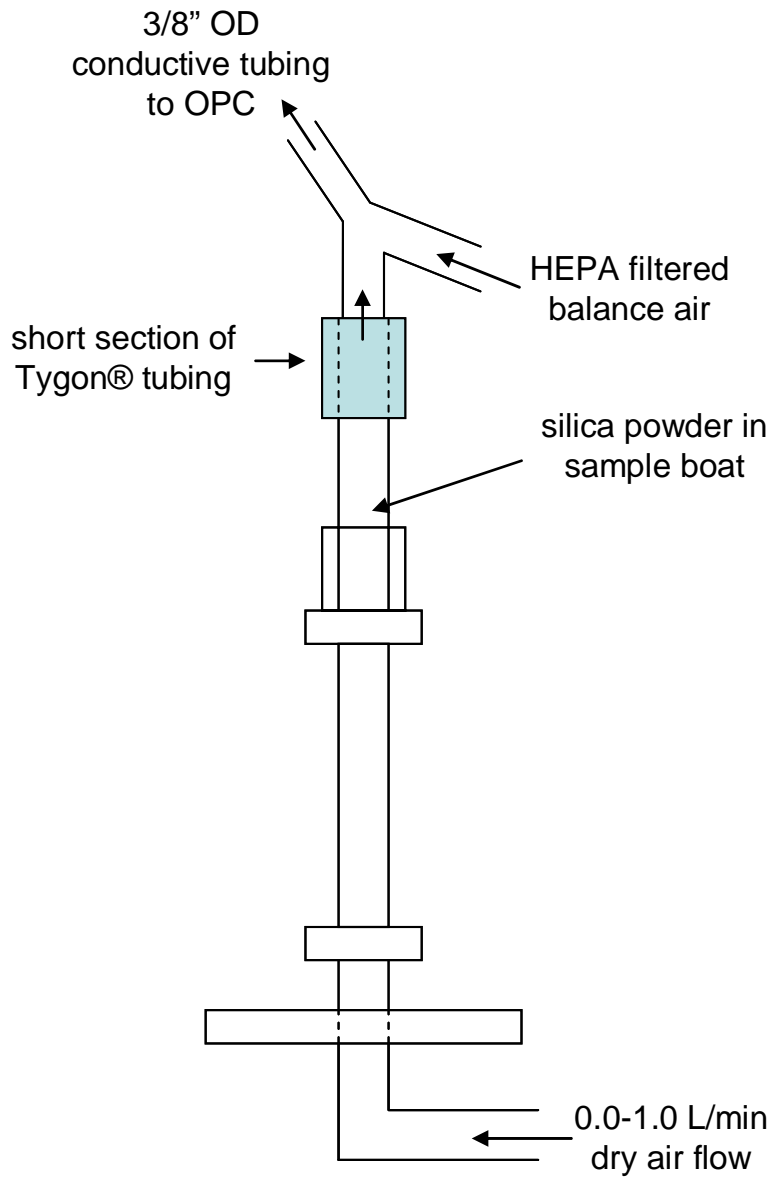

Figure 6.1. Schematic of Modified FIATA Employed for Silica Calibration Test \#4. The primary change from the typical FIATA setup is the direct connection between sample boat and OPC. 
As with hot-cell testing, the time of each flow rate increase and/or mechanical action was recorded so that each observed particle release could be correlated to a change in conditions. Release events were monitored in real time through a computer connection to the optical particle counter. Particle counts were measured and recorded at a frequency of $1 \mathrm{~Hz}$. All boat weights were made using a 5-place calibrated balance. Exposure of the powder to the atmosphere before and during testing is minimized to limit possible absorption of atmospheric moisture into the powder.

In addition to the tests above, a separate set of silica calibration measurements (called silica scoping studies) were conducted. They are different from the silica calibration tests described in the preceding paragraphs in that they 1) consist of a single end-point measurement of detection/release efficiency and 2 ) focus only on the 0 - to 900 -SCCM flow-rate range. These silica scoping studies provide a measure of calibration reproducibility.

For the silica scoping tests, approximately 50 to $100 \mathrm{mg}$ of silica powder was transferred into a preweighed sample boat. The exact powder mass added was then determined by mass difference. The sample boat with powder was then secured in the sample holder and the test apparatus assembled. The test assembly was subjected to flows spanning 100 to 900 SCCM at 100-SCCM increments. Each flow set point was held for 5 minutes, during which time the boat effluent was monitored continuously for particle release. At the end of 5 minutes, the next flow point was set and immediately engaged. The test apparatus remained assembled until the end of testing, at which point the sample boat was removed and weighed to determine the actual mass of powder released.

Silica scoping tests consisted of five experimental setups:

- Scoping Test \#1: employed the normal test setup. This test setup is equivalent to Silica Calibration \#1 and the first part of Silica Calibration \#3. No mechanical actions were performed on the test setup. As such, it provides a measure of reproducibility for these tests.

- Scoping Test \#2: employed the direct connection test setup, but with a 3-foot length of boat-toOPC conductive tubing. No mechanical actions were performed on the test setup. With the exception of the long conductive tubing, this test is similar to Silica Calibration Test \#4 (see Figure 6.1).

- Scoping Test \#3: employed the direct connection test setup, but with a 3-foot length of sample boat-to-OPC conductive tubing. This test is equivalent to Silica Scoping Test \#2, with the exception that at the end of the $900-$ SCCM set point, the sample boat was tapped to assist particle release.

- Scoping Test \#4: employed the direct connection test setup with a short ( $\sim 3$ inch) connection between sample boat and OPC. This test is equivalent to Silica Calibration Test \#4, and as such, provides a measure of that test's reproducibility.

- Scoping Test \#5: employed the direct connection test setup, but with a 3-foot length of boat-toOPC tubing. Unlike Scoping Tests \#2 and \#3, which used the typical 0.19-in. inner diameter conductive plastic tubing from TSI, this test used a stainless steel tubing section. This allowed a high-level examination of the influence of line material on particle hold-up.

For silica scoping tests, the time of each flow-rate increase and/or mechanical action was recorded so that each observed particle release could be correlated to a change in conditions. Release events were monitored in real time through a computer connection to the optical particle counter. Particle counts were 
measured and recorded at a frequency of $1 \mathrm{~Hz}$. All boat weights were made using a 5-place calibrated balance.

It should be noted that not all silica calibrations performed are reported herein. In particular, two tests were excluded because of data-quality concerns. The first excluded test was a silica calibration similar to Silica Calibration Test \#4, with the exception that it employed a 3-foot conductive tubing segment. Unlike other silica calibrations, exposure of the silica powder to the atmosphere was not well controlled during this test. As such, many of the initial test points show significant mass gain (rather than the expected loss). For example, the powder used for this test gained approximately $0.30 \mathrm{mg}$ between sample loading and the finish of the first flow set point. Moisture absorption by the powder is the suspected cause of the gain. Data are excluded because significant mass gains resulting from moisture absorption obfuscate the true release of powder from the boat.

The second excluded test was a silica calibration measurement examining releases over 100 to 1000 SCCM and was similar to Silica Calibration Test \#4. Here, a short, 3-in. direct connection between boat and OPC was employed; however, a new boat and 100 -mg powder sample was placed in the OPC after each flow set point. The use of a new boat and powder at each step required that mass losses be calculated as differential, rather than cumulative, changes. A cumulative loss could only be constructed by summing each differential loss. Because differential losses were all less than $35 \mathrm{mg}$, changes in mass were all close to the limit of detection of $10 \mathrm{mg}$ (see Section 6.2) and, as such, were of low significance. In addition, exposure of powder to the atmosphere was not well controlled and absorption of moisture was observed. For these two reasons, data corresponding to this test were excluded from this report.

\subsection{Analysis of Raw Silica Calibration Data}

Raw test data for the silica calibration include the optical particle counter data (i.e., the event and OPC count logs) as well as the sample-boat measurements, including the initial weight with powder and the weights taken after select set points. Section 3.2 describes how to analyze the OPC data.

Mass loss from the sample boat is determined by difference between the sample boat weight measurements and a given reference point. The reference point selected is the initial weight of boat and powder, as this allows the most accurate loss calculation. As such, the powder loss (or actual release) from the sample boat can be expressed as:

$$
m_{\text {loss }}^{(l)}=m_{\text {ref }}-m_{\text {boat }}^{(l)}
$$

where $m_{\text {loss }}^{(l)}$ and $m_{\text {boat }}^{(l)}$ are the loss and boat mass after event $l$, and $m_{\text {ref }}$ is the reference mass. The mass loss expressed by the above equation is cumulative. It incorporates the loss resulting from all prior flow set points and mechanical actions. This is more accurate than an event-to-event loss calculation, which references the previous event (or series of events) simply because of balance accuracy. The 5-place balance used for these measurements allowed estimates of sample boat loss to the nearest $0.01 \mathrm{mg}$. Experience and repeat mass measurements suggested an actual accuracy of $\sim 0.1 \mathrm{mg}$. As shown in the silica calibration results below, most of the changes in boat mass resulting from powder were also on the order of $0.1 \mathrm{mg}$, especially at the low flow rates (100 to $1000 \mathrm{SCCM})$. As a result, event-to-event calculations provide mass losses that are typically statistically indistinguishable from zero. While this is 
also true for the initial cumulative mass loss calculations, the cumulative mass loss eventually becomes large enough to be statistically significant.

To be directly comparable to the result of the mass loss equation, the mass detected by the OPC must also be expressed as a cumulative measurement. This is done by simply summing up the mass for each event $k$ up to the measurement of interest $l$. That is,

$$
m_{\text {detect }}^{(l)}=\sum_{k=1}^{l} m_{\text {release }}^{(k)}
$$

where $m_{\text {detect }}^{(l)}$ is the cumulative mass detected by the OPC up to event $l$. The detection efficiency, $\phi^{(l)}$, can then be expressed as the ratio of the mass detected to the mass lost,

$$
\phi^{(l)}=\frac{m_{\text {detect }}^{(l)}}{m_{\text {loss }}^{(l)}}
$$

\subsection{Silica Calibration Results}

The results for silica calibration tests 1 to 3 are presented in the graphs and tables below.

\subsubsection{Silica Calibration Test \#1}

Table 6.2 and Figure 6.2 present the results for silica test \#1, which examined counting efficiencies over flow rates between 100 and 1000 SCCM without any mechanical action. Table 6.2 suggests counting efficiencies between 0.44 and 0.79 ; the efficiency appears to increase with the flow rate of gas through the sample boat. The total material released is $\sim 0.56 \mathrm{mg}$ by boat loss and $0.44 \mathrm{mg}$ by OPC detection. In terms of the total mass of powder placed in the boat $(111.5 \mathrm{mg})$, the total release of powder relative to the total available powder is relatively small (i.e., it represents a $\sim 0.5 \%$ release of material).

Figure 6.2 shows the correlation between the cumulative mass of silica lost from the boat and that detected at the OPC for test \#1. The mass detected appears to scale quadratically with the mass lost from the boat; however, this could be an artifact of the error in the mass of silica lost from the boat. A rough approximation of global counting efficiency is made by fitting a zero intercept line to the data and measuring the slope of the resulting curve. The result indicates a global efficiency for the entire test of 0.70 . 
Table 6.2. Event-to-Event Results of Silica Calibration for Test \#1, Which Examined Counting Efficiencies over Flow Rates Between 100 and 1000 SCCM Without any Mechanical Action

\begin{tabular}{|c|c|c|c|}
\hline Sample State & $\begin{array}{l}\text { Mass Lost, } \\
\quad m_{\text {loss }} \\
{[\mathrm{mg}]}\end{array}$ & $\begin{array}{l}\text { Mass Detected, } \\
m_{\text {detect }} \\
{[\mathrm{mg}]}\end{array}$ & Efficiency, $\phi$ \\
\hline Initial boat with powder & 0.0 & 0.00 & -- -- \\
\hline Post 100-SCCM test & 0.0 & 0.00 & -- -- \\
\hline Post 200-SCCM test & 0.05 & 0.01 & -- -- \\
\hline Post 300-SCCM test & 0.05 & 0.02 & ---- \\
\hline Post 400-SCCM test & 0.02 & 0.03 & ---- \\
\hline Post 500-SCCM test & 0.0 & 0.05 & ---- \\
\hline Post $600-\mathrm{SCCM}$ test & 0.14 & 0.06 & 0.44 \\
\hline Post 700-SCCM test & 0.25 & 0.14 & 0.56 \\
\hline Post 800-SCCM test & 0.39 & 0.26 & 0.67 \\
\hline Post 900-SCCM test & 0.47 & 0.33 & 0.70 \\
\hline Post 1000-SCCM test & 0.56 & 0.44 & 0.79 \\
\hline \multicolumn{4}{|c|}{$\begin{array}{l}\text { Note: Efficiencies are undefined when the mass loss from the sample boat is } 0.0 \text { (or not measurable). } \\
\text { Mass loss below } 0.1 \mathrm{mg} \text { was considered too uncertain to use because of the uncertainty in the balance } \\
\text { reading and the susceptibility of moisture pick up and loss affecting readings. Mass increases are reported } \\
\text { as zero in this analysis. No efficiencies are calculated for mass loss below } 0.1 \mathrm{mg} . .\end{array}$} \\
\hline
\end{tabular}




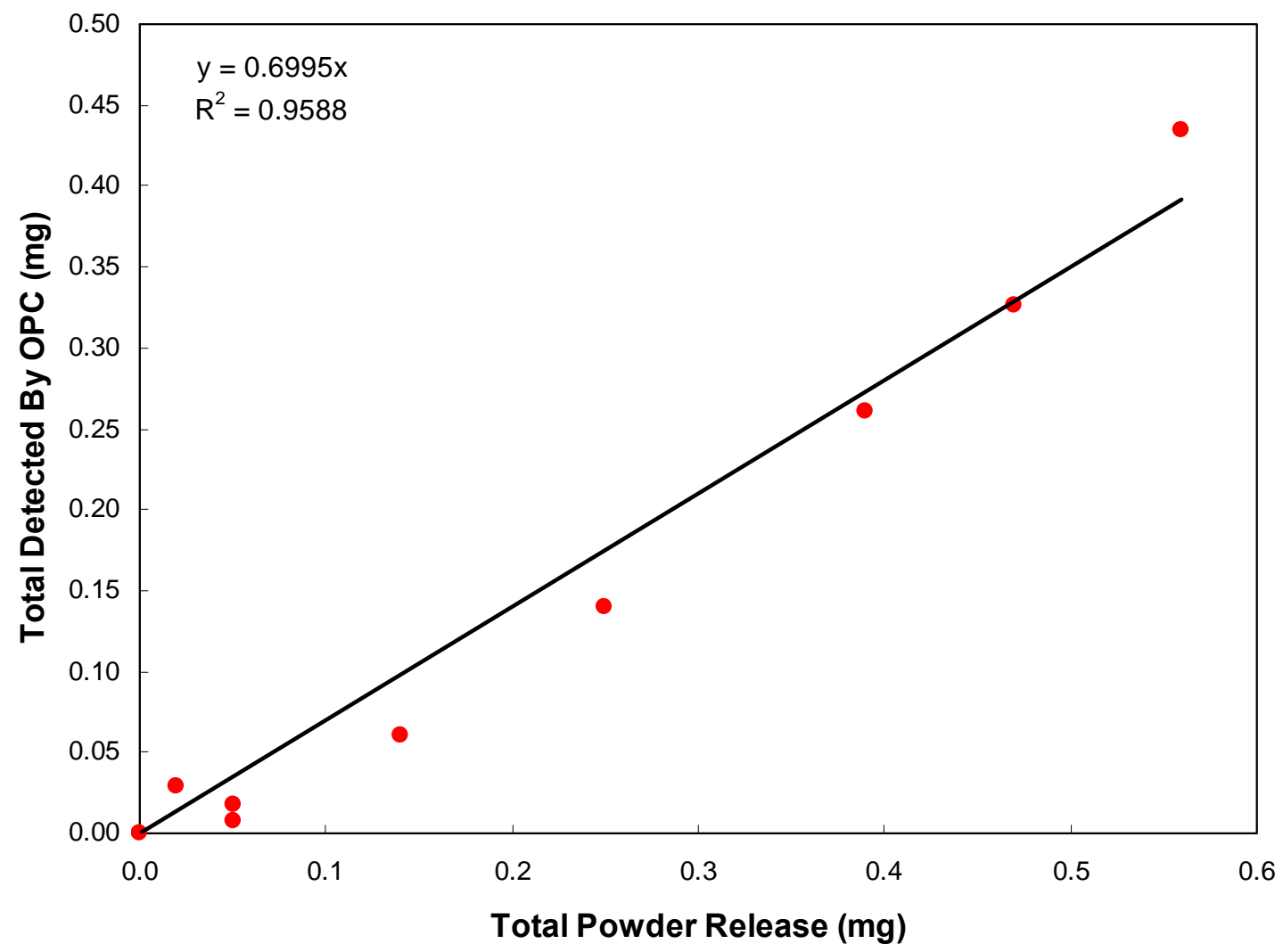

Figure 6.2. Silica Calibration Correlation Curve Showing the Mass Detected at the OPC as a Function of the Mass Lost from the Sample Boat for Silica Calibration Test \#1

\subsubsection{Silica Calibration Test \#2}

Table 6.3 and Figure 6.3 present the results for silica test \#2, which examined counting efficiencies over flow rates between 100 and 1000 SCCM with mechanical action (i.e., tapping of the enclosure housing during the first 30 seconds of the 5-minute flow setting). Table 6.3 suggests counting efficiencies between 0.21 and 0.25 ; as before, the efficiency appears to increase with the flow rate of gas through the sample boat. Tapping of the sample boat housing has had two effects: 1) the efficiency of the collector has been reduced by a factor of 2 to 3 and 2) the amount of material released has increased an order of magnitude. The increase in magnitude of the boat loss most likely results from fragmentation of the powder aggregates as a result of mechanical agitation. Increased fragmentation could potentially lead to a larger size range of material released from the boat. Increased release and settling of large particle aggregates could be the cause of the reduced counting efficiency. On the other hand, the reduced counting efficiency could simply result from a better estimate of the mass loss and mass detected (because of their increased magnitude relative to the balance error of $\sim 0.1 \mathrm{mg}$ ). In terms of the total mass of powder placed in the boat $(107.8 \mathrm{mg})$, the total release of powder $(7.9 \mathrm{mg})$ relative to the total available powder is $7 \%$ and is much larger than before. 
Table 6.3. Event-to-Event Results of Silica Calibration for Test \#2, Which Examined Counting Efficiencies over Flow Rates Between 100 and 1000 SCCM with Mechanical Action (i.e., Tapping of the Enclosure Housing During the First 30 Seconds of the 5 Minute Flow Setting)

\begin{tabular}{||l|c|c|c||}
\hline \multicolumn{1}{|c|}{ Sample State } & $\begin{array}{c}\text { Mass Lost, } \\
\boldsymbol{m}_{\text {loss }} \\
{[\mathbf{m g}]}\end{array}$ & $\begin{array}{c}\text { Mass Detected, } \\
\boldsymbol{m}_{\text {detect }} \\
{[\mathbf{m g}]}\end{array}$ & Efficiency, $\boldsymbol{\phi}$ \\
\hline Initial boat with powder & 0.0 & 0.00 & --- \\
\hline $\begin{array}{l}\text { Post 100- to 800-SCCM tests with } \\
\text { tapping }\end{array}$ & 4.0 & 0.83 & 0.21 \\
\hline $\begin{array}{l}\text { Post 900- to 1000-SCCM tests with } \\
\text { tapping }\end{array}$ & 7.9 & 1.98 & 0.25 \\
\hline
\end{tabular}

Figure 6.3 shows the correlation between the cumulative mass of silica lost from the boat and that detected at the OPC for test \#2. As before, the mass detected appears to show a slight upward concavity with the mass lost from the boat. A best-fit of the correlation using a zero intercept line suggests a global efficiency of the process to be approximately 0.24 .

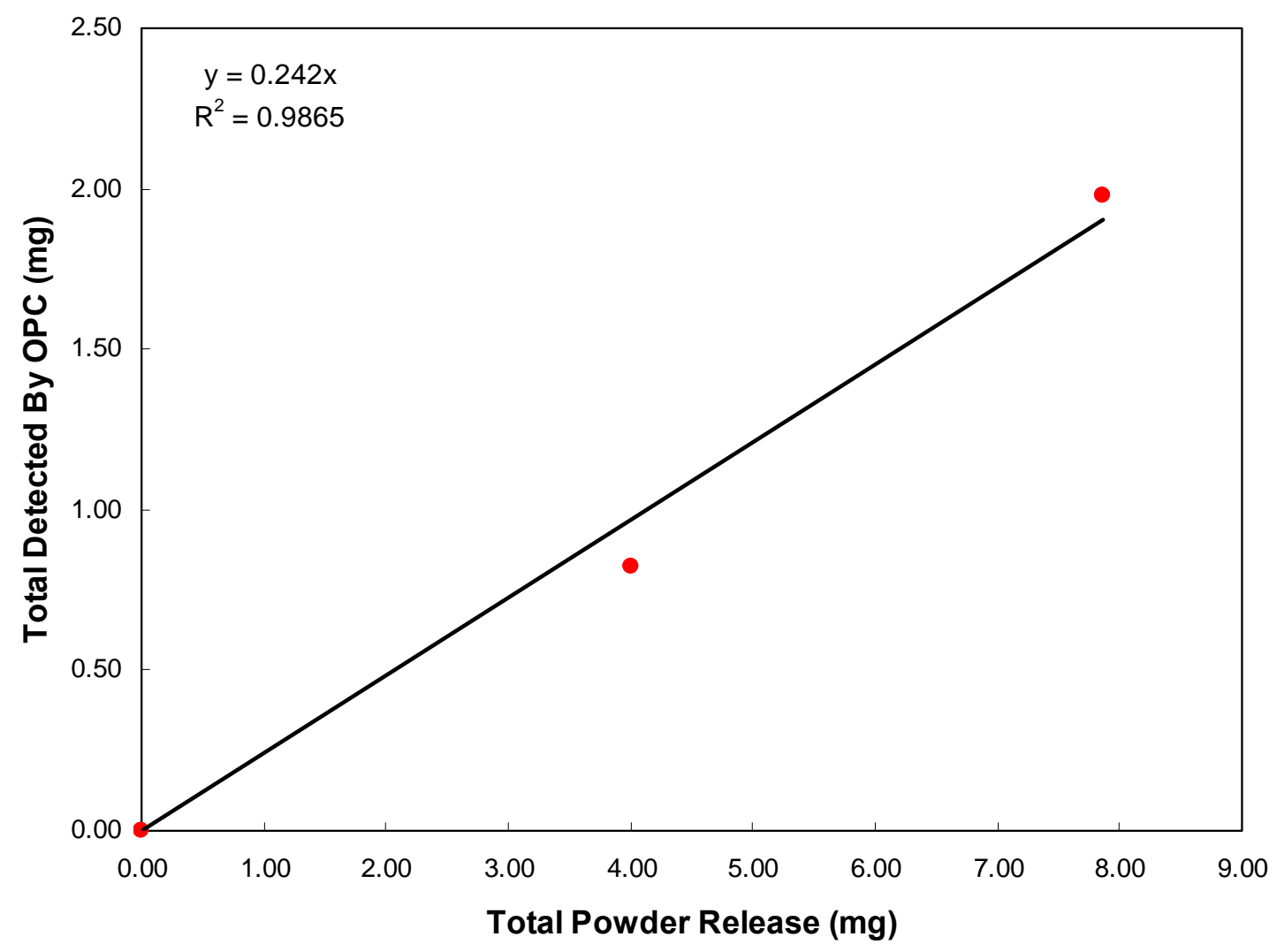

Figure 6.3. Silica Calibration Correlation Curve Showing the Mass Detected at the OPC as a Function of the Mass Lost from the Sample Boat Silica Calibration Test \#1 


\subsubsection{Silica Calibration Test \#3}

Table 6.4 and Figure 6.4 present the results for silica test \#3, which examined counting efficiencies over flow rates between 100 and 1000 SCCM with mechanical action (i.e., tapping of the enclosure housing during the first 30 seconds of the 5-minute flow setting). The counting efficiencies shown in Table 6.4 suggest relatively low efficiencies between 0.076 and 0.11 . The efficiency over the 100 - to 1000 -SCCM range is lower than the ramped chamber test, and the release is about a factor of 3 larger. The only difference in the $<1000$-SCCM region in tests 1 and 3 is the flow-controller exchange conducted in test \#3. The exchange causes a transient spike in flow rate, which could force aggregates out of the boat that settle out in the chamber before being sampled by the collection system. It should be noted that the material release occurring in test \#3 at flows between 100 to 1000 SCCM is still less than the tapped test.

The reduced counting efficiency between 1 and 3 SLM at higher flow rates appear to result from flow splitting. It should be noted that the amount of material released by flow alone above 3 SLM is nearly two orders of magnitude greater than the maximal release in test \#1 and a factor of 2 larger than the maximal release in test \#2. Tapping of the housing at 3 SLM causes a near $100 \%$ release of material from the boat. Specifically, the total release here is $139.5 \mathrm{mg}$, while the amount of material available for release (i.e., that added to the sample boat) was $143.0 \mathrm{mg}$. This represents a boat release of $\sim 98 \%$ of the material by mass. While much of the material was released, only $7.6 \%$ of the material was detected at OPC. This suggests that much of the material release either stuck to the FIATA walls or settled to the bottom of the sample chamber. This was confirmed by opening the sample chamber, whereupon, the silica powder was observed to coat the lower enclosure surfaces.

Table 6.4. Event-to-Event Results of Silica Calibration for Test \#3, Which Examined Counting Efficiencies Using Flow Set Points and Mechanical Actions Similar to Those Used in Hot Cell Testing

\begin{tabular}{||l|c|c|c||}
\hline \multicolumn{1}{|c|}{ Sample State } & $\begin{array}{c}\text { Mass Lost, } \\
\boldsymbol{m}_{\text {loss }} \\
{[\mathbf{m g}]}\end{array}$ & $\begin{array}{c}\text { Mass Detected, } \\
\boldsymbol{m}_{\text {detect }} \\
{[\mathbf{m g}]}\end{array}$ & Efficiency, $\phi$ \\
\hline Initial boat with powder & 0.0 & 0.00 & ---- \\
\hline $\begin{array}{l}\text { Flows from 100 to 1000 SCCM at } \\
\text { 100-SCCM intervals with a } \\
\text { controller exchange at 200 SCCM }\end{array}$ & 1.6 & 0.18 & 0.11 \\
\hline $\begin{array}{l}\text { Flows from 1 to 3 SLM at 1-SLM } \\
\text { increments }\end{array}$ & 16.2 & 1.32 & 0.082 \\
\hline $\begin{array}{l}\text { Flow of 3 SLM with a 30-second } \\
\text { housing tap }\end{array}$ & 139.5 & 10.59 & 0.076 \\
\hline
\end{tabular}

Figure 6.4 shows the correlation between the cumulative mass of silica lost from the boat and that detected at the OPC for test \#3. The mass detected appears to scale linearly with the mass lost from the boat, but this could simply be an artifact of the magnitude of the final point. A best-fit of the correlation using a zero intercept line suggests a global efficiency of the process to be approximately 0.076 . 


\subsubsection{Silica Calibration Test \#4}

Table 6.5 and Figure 6.5 present the results for silica test \#4, which examined counting efficiencies using a direct connection to the OPC over flow rates between 100 and 1000 SCCM without any mechanical action. The direct connection and use of flow rates below 1 SLM eliminates both settling and flow splitting as a source of material loss. As a result, loss is limited to particle sticking

Surprisingly, the counting efficiencies using the direct connection, which show a maximum of 0.41 , are less than that observed in the equivalent chamber tests. However, the amount of material released during test \#4 ( $\sim 3.9 \mathrm{mg}$ ) is also an order of magnitude greater than in test \#1 ( $\sim 0.6 \mathrm{mg})$, so the difference may simply be a result of better accuracy relative to the limit of mass measurement $(\sim 0.1 \mathrm{mg})$. Relative to the total mass available for release $(91.1 \mathrm{mg})$, the actual release represents about $4.3 \%$ of the total material. It is suspected that the increased release observed in test $\# 4$ occurs as a result of the additional mechanical agitation required to remove the Y-junction when weighing the sample boat. This results in a direct disturbance of the silica powder in the boat and may cause powder fragmentation. Because of these differences, it is difficult to directly compare the results from test \#1 and test \#4.

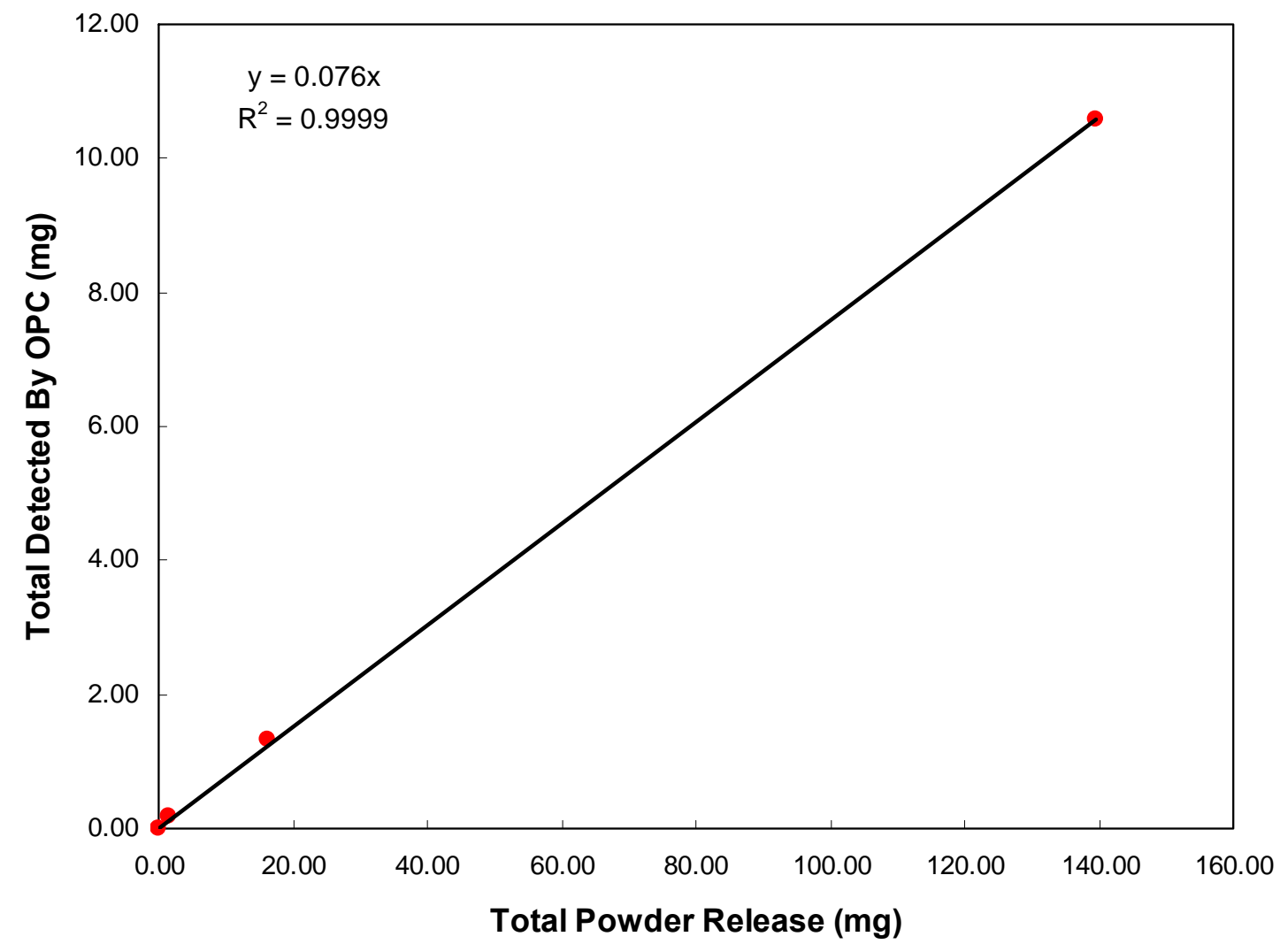

Figure 6.4. Silica Calibration Correlation Curve Showing the Mass Detected at the OPC as a Function of the Mass Lost from the Sample Boat Silica Calibration Test \#3 
Table 6.5. Event-to-Event Results of Silica Calibration for Test \#4, Which Employed a Direct Connection Between Sample Boat and OPC

\begin{tabular}{||l|c|c|c||}
\hline \multicolumn{1}{|c|}{ Sample State } & $\begin{array}{c}\text { Mass Lost, } \\
\boldsymbol{m}_{\text {loss }} \\
{[\mathbf{m g}]}\end{array}$ & $\begin{array}{c}\text { Mass Detected, } \\
\boldsymbol{m}_{\text {detect }} \\
{[\mathbf{m g}]}\end{array}$ & Efficiency, $\boldsymbol{}$ \\
\hline Initial boat with powder & 0.0 & 0.00 & ---- \\
\hline Post 100-SCCM test & 0.20 & 0.00 & 0.00 \\
\hline Post 200-SCCM test & 0.52 & 0.003 & 0.01 \\
\hline Post 300-SCCM test & 0.37 & 0.010 & 0.03 \\
\hline Post 400-SCCM test & 0.45 & 0.061 & 0.14 \\
\hline Post 500-SCCM test & 0.89 & 0.188 & 0.21 \\
\hline Post 600-SCCM test & 0.81 & 0.287 & 0.35 \\
\hline Post 700-SCCM test & 1.60 & 0.429 & 0.27 \\
\hline Post 800-SCCM test & 2.38 & 0.823 & 0.35 \\
\hline Post 900-SCCM test & 3.19 & 1.23 & 0.39 \\
\hline Post 1000-SCCM test & 3.91 & 1.59 & 0.41 \\
\hline Note: efficiencies are undefined when the mass loss from the sample boat is 0.0 (or not measurable). \\
\hline
\end{tabular}

Figure 6.5 shows the correlation between the cumulative mass of silica lost from the boat and that detected at the OPC for test \#4. As with test \#1, the mass detected appears to scale quadratically with the mass lost from the boat, however. A zero-intercept linear fit of the cumulative mass correlation suggests a global efficiency of 0.36 . 


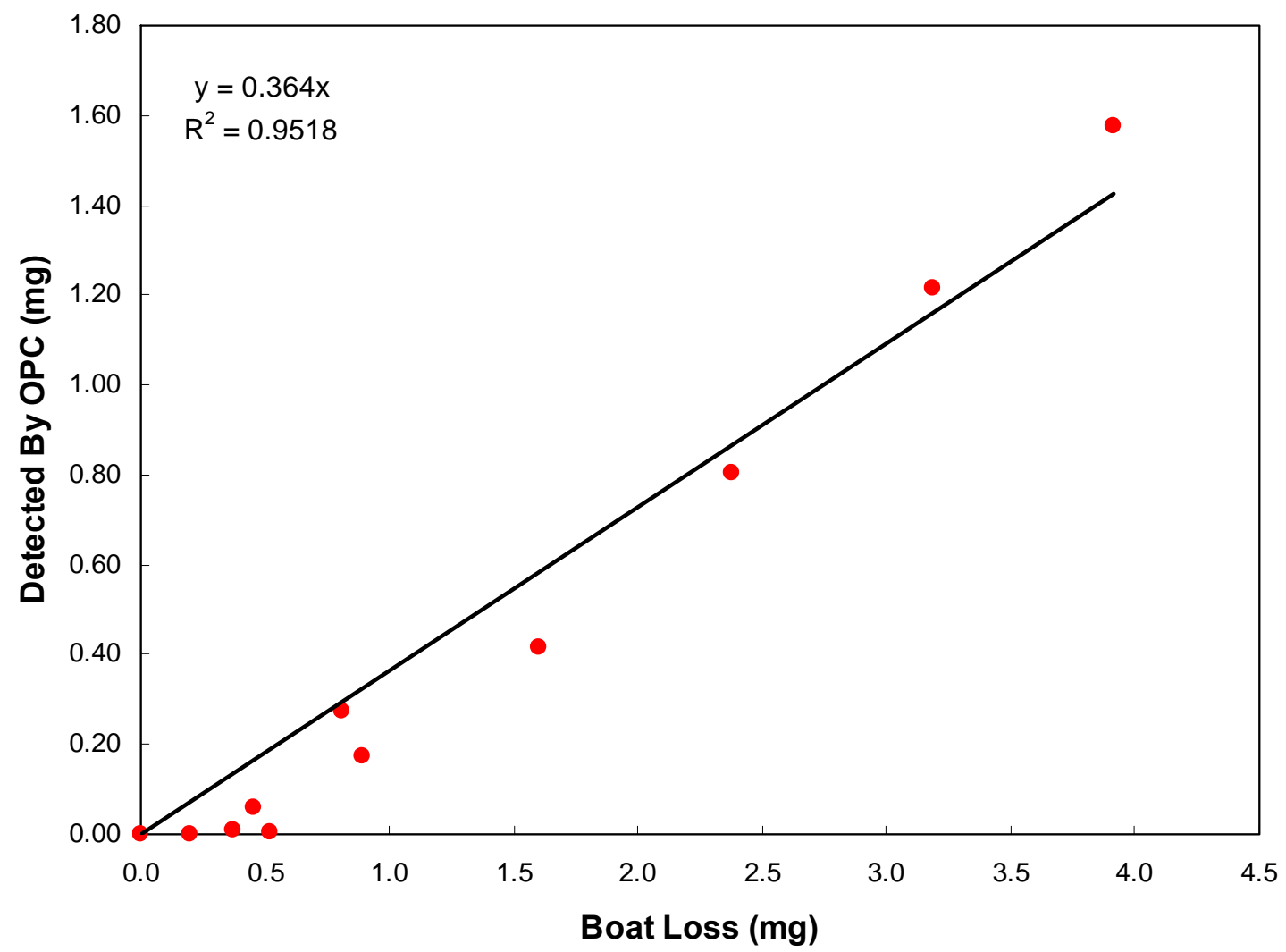

Figure 6.5. Silica Calibration Correlation Curve Showing the Mass Detected at the OPC as a Function of the Mass Lost from the Sample Boat Silica Calibration Test \#4

\subsubsection{Examination of Release Particle Size as a Function of Gas Flow Rate}

Tests \#1 and \#4 examine particle releases over a continuous range of gas flow rates without any mechanical action, and as such, provide an opportunity to see if the size of particle released varies as a function of flow rate. Based on Stoke's predictions alone, it is expected that small particles would represent the majority released at low flow rates and large particles the majority at high flow rates. On the other hand, the smallest particles also show the highest sticking potential (based on their higher surface area-to-volume ratio).

Figure 6.6 shows the PSD, in terms of $\mathrm{d}(10), \mathrm{d}(50)$, and $\mathrm{d}(90)$, as a function of gas flow rates for silica calibration tests \#1 and \#4. In both cases, the particle size does not appear to show any predictable trend with the flow rate. This could suggest that the flow rate required to fragment particles off of the powder mass resting in the sample boat is sufficient to suspend the majority of particles. A comparison of the distribution observed in tests \#1 and \#4 finds that both are roughly equivalent. This suggests that for these particular tests, the sample chamber setup did not have a profound impact on the size of material detected. 


\subsubsection{Summary of Silica Calibration Test Results}

Table 6.6 provides a summary of the fractional powder releases and the estimated global detection efficiencies determined for each test setup. From silica calibration testing results presented in this table, it may be concluded that the combination of high flow and mechanical action leads to complete release of particulate matter (as indicted by the results of Test \#3). At flows lower than 1 SLM, only a fraction of the material is released from the sample boat. Depending on the addition of mechanical agitation of the powder, the release represents anywhere from $0.5 \%$ to $7 \%$ of the total material in the sample boat.

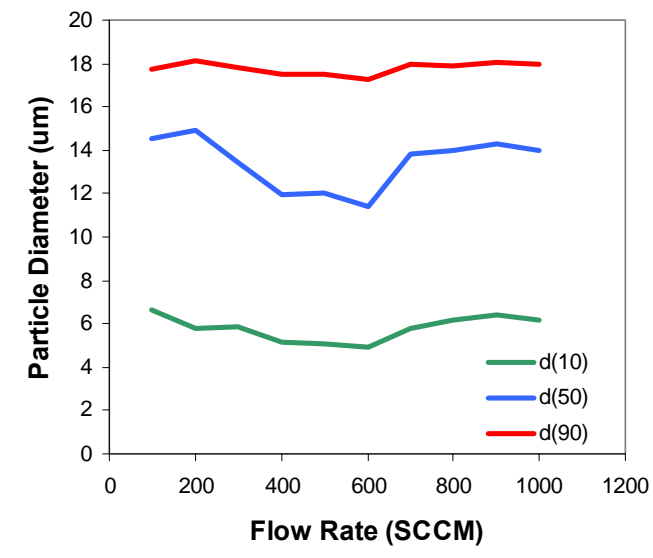

(A)

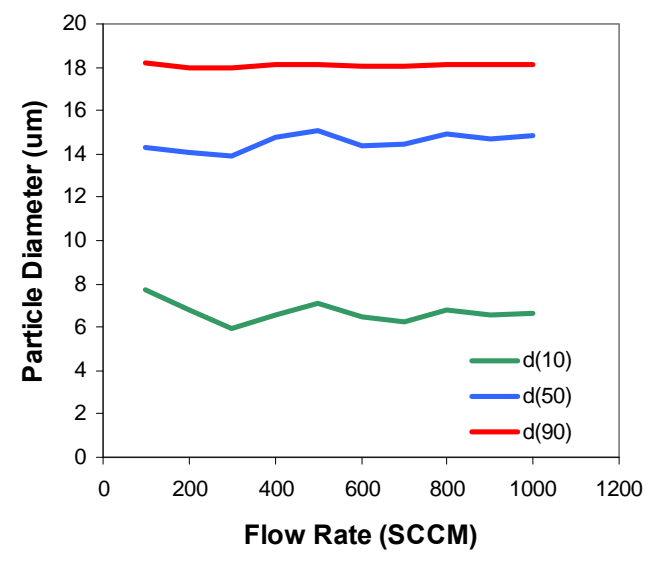

(B)

Figure 6.6. Particle Size as a Function of Flow Rate for (A) Silica Calibration Test \#1, Using the Typical FIATA Setup over Flow Rates Between 100 to 1000 SCCM and (B) Silica Calibration Test \#4, Using a Direct Connection Between Sample Boat and OPC over 100 to 1000 SCCM

Table 6.6. Summary of Silica Calibration Test Results

\begin{tabular}{|c|l|c|c|c|}
\hline Test & Conditions & $\begin{array}{c}\text { Total Mass of } \\
\text { Powder [mg] }\end{array}$ & $\begin{array}{c}\text { Fraction } \\
\text { Released }\end{array}$ & $\begin{array}{c}\text { Estimated Global } \\
\text { Detection Efficiency }\end{array}$ \\
\hline 1 & $\begin{array}{l}\text { Normal test setup: 100-1000 SCCM; } \\
\text { no tapping }\end{array}$ & 111.5 & $0.5 \%$ & 0.70 \\
\hline 2 & $\begin{array}{l}\text { Normal test setup: } 100-1000 \text { SCCM; } \\
\text { with tapping }\end{array}$ & 107.8 & $7.3 \%$ & 0.24 \\
\hline 3 & $\begin{array}{l}\text { Normal test setup: 100-3000 SCCM; } \\
\text { with tapping and flow controller } \\
\text { exchange }\end{array}$ & 143.0 & $97.5 \%$ & 0.076 \\
\hline 4 & $\begin{array}{l}\text { Direct OPC connection: } 100-1000 \\
\text { SCCM }\end{array}$ & 91.1 & $4.3 \%$ & 0.36 \\
\hline
\end{tabular}

The global counting efficiency of the measurements above appears to depend on the experimental setup, flow conditions, and mechanical actions employed. Efficiencies ranged from 0.70 in tests focusing only on the low range of flow rates down to 0.076 in tests with flows greater than 1 SLM and mechanical agitation of the sample enclosure. 


\subsubsection{Summary of Silica Scoping Test Results}

Silica scoping tests provide a measure of silica calibration reproducibility. Table 6.7 provides the raw release results for the silica scoping studies. Table 6.8 provides a summary of the fractional powder releases and the estimated global detection efficiencies determined for each scoping test setup.

Comparing equivalent tests between scoping and calibration studies produces mixed results. Specifically, the release in scoping test \#1 (17.7\%) is much higher than the release in silica calibration \#1 $(0.5 \%)$ and slightly higher than in the first part of silica calibration \#3 (11.3\%). On the other hand, the release in scoping test \#4 (2.7\%) is somewhat lower than the release in silica calibration \#4 (4.3\%).

With regard to the global counting efficiency, the range observed in the scoping tests was 0.051 to 0.43 . While the overall range of counting efficiencies observed is similar to that measured in the calibration results, comparing similar tests shows substantial test-to-test differences. Specifically, the counting efficiency observed in the scoping studies for the normal test setup over 100 to 900 SCCM (i.e., Test \#1) was 0.065 . This efficiency is about one order of magnitude lower than that observed in the equivalent silica calibration testing (Test \#1 0.70).

Table 6.7. Summary of Silica Scoping Test Results

\begin{tabular}{|c|l|c|c|c||}
\hline Test & Conditions & $\begin{array}{c}\text { Powder Added } \\
\text { to Boat [mg] }\end{array}$ & $\begin{array}{c}\text { Powder Release } \\
\text { from Boat [mg] }\end{array}$ & $\begin{array}{c}\text { Powder Detected } \\
\text { at OPC [mg] }\end{array}$ \\
\hline 1 & $\begin{array}{l}\text { Normal test setup: 100-900 SCCM; } \\
\text { no tapping }\end{array}$ & 105.0 & 18.6 & 1.20 \\
\hline 2 & $\begin{array}{l}\text { Direction connection with 3-foot } \\
\text { conductive tubing: 100-900 SCCM; } \\
\text { no tapping }\end{array}$ & 77.5 & 20.1 & 4.03 \\
\hline 3 & $\begin{array}{l}\text { Direction connection with 3-foot } \\
\text { conductive tubing: 100-900 SCCM; } \\
\text { with tapping }\end{array}$ & 71.9 & 62.7 & \\
\hline 4 & $\begin{array}{l}\text { Direction connection with 3-in. } \\
\text { conductive tubing: 100-900 SCCM; } \\
\text { no tapping }\end{array}$ & 52.0 & 1.4 & 0.60 \\
\hline 5 & $\begin{array}{l}\text { Direction connection with 3-foot } \\
\text { stainless steel tubing: 100-900 } \\
\text { SCCM; with tapping }\end{array}$ & 58.7 & 0.9 & 0.13 \\
\hline
\end{tabular}


Table 6.8. Summary of Silica Scoping Test Results

\begin{tabular}{|c|l|c|c|c||}
\hline Test & Conditions & $\begin{array}{c}\text { Total Mass of } \\
\text { Powder [mg] }\end{array}$ & $\begin{array}{c}\text { Fraction } \\
\text { Released }\end{array}$ & $\begin{array}{c}\text { Estimated Global } \\
\text { Detection Efficiency }\end{array}$ \\
\hline 1 & $\begin{array}{l}\text { Normal test setup: 100-900 SCCM; } \\
\text { no tapping }\end{array}$ & 105.0 & $17.7 \%$ & 0.065 \\
\hline 2 & $\begin{array}{l}\text { Direction connection with 3-foot } \\
\text { conductive tubing: 100-900 SCCM; } \\
\text { no tapping }\end{array}$ & 77.5 & $25.9 \%$ & 0.052 \\
\hline 3 & $\begin{array}{l}\text { Direction connection with 3-foot } \\
\text { conductive tubing: 100-900 SCCM; } \\
\text { with tapping }\end{array}$ & 71.9 & $87.1 \%$ & 0.079 \\
\hline 4 & $\begin{array}{l}\text { Direction connection with 3-in. } \\
\text { conductive tubing: 100-900 SCCM; } \\
\text { no tapping }\end{array}$ & 52.0 & $2.7 \%$ & 0.43 \\
\hline 5 & $\begin{array}{l}\text { Direction connection with 3-foot } \\
\text { stainless steel tubing: 100-900 } \\
\text { SCCM; with tapping }\end{array}$ & 58.7 & $1.5 \%$ & 0.15 \\
\hline
\end{tabular}

\subsubsection{Estimate of Global Counting Efficiency}

The goal of silica calibration testing was to provide an estimate for the counting efficiency (i.e., material holdup, settling, and splitting) for the actual fuel testing as well as a point of comparison for material release. The estimation is complicated by the fact that the disparate results between the calibration and scoping tests suggest that both the fraction of powder released and the counting efficiency are highly variable, even for similar test setups. The cause of these differences is unknown, but may include, among other things, differences in the geometry of powder resting in the sample boat and differences in handling and mechanical action as it is inserted into the sample holder and as the test apparatus is assembled.

Despite the high variability, the range of counting efficiencies for silica appears to fall between 0.052 and 0.43 . For the purpose of estimating the fractional release in the spent-fuel tests, a global efficiency based on the silica calibration and scoping tests most closely approximating the SAL FIATA experiments will be chosen. As such, the selection is limited to silica calibration Tests 1 to 3 and silica scoping Test 1 . The most conservative global efficiency observed in these tests is 0.065 . It is conservative in that it is the lowest efficiency observed for these tests, and as such, provides an upper bound of the mass release from the boat (or rod in segment tests). The conditions under which this 0.065 efficiency were measured were 100 to 900 SCCM with no mechanical actions. All tests performed under similar conditions showed higher counting efficiencies. Tests employing higher flows and mechanical action typically showed similar (but slightly higher) efficiencies. As such, the 0.065 is bounding for all flow and mechanical action conditions.

When compared to release fractions observed for oxidized fuel powder, the bounding 0.065 release appears to underestimate fuel release by two orders of magnitude. It is postulated that this discrepancy is a result of differences in the physical properties between the silica powder and actual oxidized fuel powder. Specific properties that could yield different counting efficiencies between silica and actual fuel 
include, but are not limited to, particle sticking potential, the tendency of the powder to form particle aggregates, and density. Regarding sticking potential, fuel particles are known to charge as a result of radioactive decay, which may lead to an increased tendency for those particles to stick to oppositely charged surfaces. Silica particles, which are non-radioactive, are not subject to this phenomenon. In addition, fuel particles $(\sim 8.35 \mathrm{~g} / \mathrm{cc})$ are much denser than silica particles $(\sim 2.5 \mathrm{~g} / \mathrm{cc})$ and should settle more rapidly in flowing air streams. Based on the expected increase in fuel sticking potential and higher fuel density, the reduced counting efficiency observed for fuel relative to silica is not surprising. Indeed, the reduced counting efficiencies suggest that oxidized fuel particles are more difficult to suspend in air than the silica powder used for calibration. As such, the silica powder could provide a useful point of reference for future dispersability studies.

Despite the ability to estimate global counting efficiencies and apply them to gain a better understanding of fuel release, the current test setup was far from ideal. Questions about the tendency of the fuel to stick to surfaces and settle out of the air-stream before reaching intended collection surfaces remain. For this reason, additional fuel-release studies are planned for fiscal year 2008 at PNNL. These tests will employ a horizontal flow configuration. Unlike the current tests, the FY08 setup will consist of a single tube through which air is flowed at a controlled rate over a powder resting either on the bottom of the tube or on a fuel support receptacle inside the tube. A series of dead-end collection filters will capture any fuel entrained in the air. A tube liner will be used to collect fuel that settles out before reaching the dead-end filters. This setup addresses two problems with the current setup:

- Because the setup does not use an OPC or cascade impactor, the need for balance air (and flow annulus) is eliminated. The result is that there are no changes in the velocity of gas suspending the fuel.

- Collecting both settled fuel (on the tube liner) and airborne fuel (on the dead-end filters) provides more closure on the fuel mass balance. This provides more detail on how much of the fuel settles out and how quickly it settles out.

Overall, we believe that the FY08 setup will answer many of the outstanding questions the current study could not answer. 


\subsection{Particle Momentum Calculations}

The purpose of this chapter is to provide a theoretical basis for the motion of particles within the test apparatus. This basis provides general insight into the results and discussions presented in this report. Additionally, this chapter explicitly addresses the concern that the accelerated air flow at the frits used in the powder tests may have transferred excess momentum to powder particles, which would possibly have a noticeable effect on the results.

\subsection{System Description}

In the powder test experiments presented in this report, a powder initially at rest is subjected to an upward-flowing air stream. To achieve this condition, the powder is initially placed inside a sample boat, the bottom of which is actually a frit with a 0.5 -micron pore size. This frit provides a stable support for the particles while allowing the air to flow up through the powder sample. After the upward air flow is initialized, the particles that are suspended within the flow are carried out of the sample boat to an optical particle counter where the PSD and quantity of particles are measured. To better understand the physical conditions of the experiment and to better understand the results recorded, particle momentum balances should be performed. The objective of these balances is to determine what particles will be removed from the sample boat by the air flow. The calculations will show that for each flow condition considered, there is a maximum theoretical particle size that could be removed. As these calculations are necessarily of a theoretical nature, each consideration and assumption made will be of a conservative nature and will be openly declared for future manipulation should it be determined that less conservative answers are necessary for applicability.

The problem description must begin with the flow parameters. The bottom of the sample boat (the end of the boat containing the frit) is connected to a pressurized gas cylinder by a pipe. A flow controller is placed inline between the cylinder and the sample boat to manipulate the volumetric flow rate of air used to suspend the test particles. After leaving the flow controller, the air stream will flow along the pipe in a bulk-fluid motion. Once the air stream reaches the frit, however, it will be divided into several jets emerging on the top side of the frit from the pores. Before considering the effects of this phenomenon, it is important to quantify the parameters associated with it. The parameters associated with the frit are:

Diameter, $\underline{\mathrm{D}}=0.374$ inches $=0.950 \mathrm{~cm}$

Height, $\mathrm{H}=0.062$ inches $=0.157 \mathrm{~cm}$

Total Volume, $\mathrm{V}_{\text {total }}=\pi \underline{D}^{2} \mathrm{H} / 4=(\pi / 4)(0.950 \mathrm{~cm})^{2}(0.157 \mathrm{~cm})=0.112 \mathrm{~cm}^{3}$

Mass, $\mathrm{M}=0.7094 \mathrm{~g}$

The frit is made of stainless steel having the following properties:

Density of Steel, $\rho=7.5 \mathrm{~g} / \mathrm{cm}^{3}$

Solid Volume of Filter $=V_{\text {filter }}=M / \rho=0.7094 \mathrm{~g} / 7.5 \mathrm{~g} / \mathrm{cm}^{3}=0.0946 \mathrm{~cm}^{3}$

Void Volume, $V_{\text {void }}=V_{\text {total }}-V_{\text {filter }}=0.112 \mathrm{~cm}^{3}-0.0946 \mathrm{~cm}^{3}=0.017 \mathrm{~cm}^{3}$ 
Void Area $=\mathrm{A}_{\text {Void }}=\mathrm{V}_{\text {Void }} / \mathrm{H}=0.017 \mathrm{~cm}^{3} / 0.157 \mathrm{~cm}=0.108 \mathrm{~cm}^{2}$

Total Filter Area, $\mathrm{A}_{\text {total }}=(\pi / 4) \underline{D}^{2}=(\pi / 4)(0.950 \mathrm{~cm})^{2}=0.709 \mathrm{~cm}^{2}$

Fractional Void Area, $\mathrm{f}_{\text {void }}=\mathrm{A}_{\text {Void }} / \mathrm{A}_{\text {total }}=0.108 \mathrm{~cm}^{2} / 0.709 \mathrm{~cm}^{2}=0.153$

These frit parameter values can be used to determine the behavior of the air flow through the frit. Treating the frit pore alignment as a square array, the closest pore spacing, $\lambda_{\min }$, can be determined to be

$$
\lambda_{\min }=d_{p} \sqrt{\frac{\pi}{4 f_{\text {void }}}}=0.5 \mu \mathrm{m} \sqrt{\frac{\pi}{4(0.153)}}=1.13 \mu \mathrm{m}
$$

where $d_{p}$ is the pore diameter, and $f_{\text {void }}$ is the fractional void area. The farthest pore spacing can be determined to be

$$
\lambda_{\max }=\sqrt{2} \lambda_{\min }=\sqrt{2}(1.13 \mu m)=1.60 \mu m
$$

The dispersion of the gas jet exiting each pore occurs through mixing with the gas surrounding the pore and with the jets exiting adjacent pores. Since the maximum distance between pores is $1.6 \mu \mathrm{m}$, a conservative value of $10 \mu \mathrm{m}$ can be used for the radial mixing length. This conservative value allows the model to account for other possible pore arrangements that may have larger pore distances. The slowest mechanism of radial dispersion of the air jet is diffusion. Assuming a conservatively low binary diffusivity of $1 \times 10^{-5} \mathrm{~m}^{2} / \mathrm{s}$ (Deen 1998), the time scale for mixing can be determined to be

$$
t=\frac{L^{2}}{D}=\frac{\left(1 \times 10^{-5} \mathrm{~m}\right)^{2}}{1 \times 10^{-5} \mathrm{~m}^{2} / \mathrm{s}}=1 \times 10^{-5} \mathrm{~s}
$$

where $L$ is the radial mixing length, and $D$ is the diffusivity.

The height at which the post-frit air jets can be considered to disperse back into a bulk flow can be determined conservatively to be the product of the time scale calculated in Eq. (7.3) and the post-frit velocity. Table 7.1 has been created to quantify the values of the post-frit velocities relative to the superficial velocities for various air volumetric flow rates. In Table 7.1, the "superficial velocity" is the velocity at which the air would flow through the sample boat if there were no frit. According to Table 7.1, and using the mixing time calculated in Eq. (7.3), the air flow would return to a superficial velocity 1.5 micrometers above the frit for an air volumetric flow rate of 100 SCCM and would return to a superficial velocity 46.1 micrometers above the frit for a volumetric flow rate of 3000 SCCM. It is important to remember that Eq. (7.3) is an orderof-magnitude relation and should be used only for estimations. This point will be addressed later in this section. In Table 7.1, the superficial velocity is calculated with the equation

$$
V_{\text {sup }}=\frac{4 Q}{\pi \underline{D}^{2}}
$$


where $Q$ is the volumetric flow rate, and the post-frit velocity is calculated with the equation

$$
V_{p f}=\frac{V_{\text {sup }}}{f_{\text {void }}}
$$

Table 7.1. Air Flow Rates

\begin{tabular}{|c|c|c||c|c|c||c|c|c||}
\hline $\begin{array}{c}\text { Volumetric } \\
\text { Flow Rate } \\
\text { SCCM }\end{array}$ & $\begin{array}{c}\text { Superficial } \\
\text { Velocity } \\
\mathbf{m} / \mathbf{s}\end{array}$ & $\begin{array}{c}\text { Post- } \\
\text { Frit } \\
\text { Velocity } \\
\mathbf{m} / \mathbf{s}\end{array}$ & $\begin{array}{c}\text { Volumetric } \\
\text { Flow Rate } \\
\text { SCCM }\end{array}$ & $\begin{array}{c}\text { Superficial } \\
\text { Velocity } \\
\mathbf{m} / \mathbf{s}\end{array}$ & $\begin{array}{c}\text { Post- } \\
\text { Frit } \\
\text { Velocity } \\
\mathbf{m} / \mathbf{s}\end{array}$ & $\begin{array}{c}\text { Post- } \\
\text { Folumetric } \\
\text { SCCM Rate }\end{array}$ & $\begin{array}{c}\text { Superficial } \\
\text { Velocity } \\
\mathbf{m} / \mathbf{s}\end{array}$ & $\begin{array}{c}\text { Frit } \\
\text { Velocity } \\
\mathbf{m} / \mathbf{s}\end{array}$ \\
\hline 100 & 0.02 & 0.15 & 1100 & 0.26 & 1.69 & 2100 & 0.49 & 3.23 \\
\hline 200 & 0.05 & 0.31 & 1200 & 0.28 & 1.84 & 2200 & 0.52 & 3.38 \\
\hline 300 & 0.07 & 0.46 & 1300 & 0.31 & 2.00 & 2300 & 0.54 & 3.53 \\
\hline 400 & 0.09 & 0.61 & 1400 & 0.33 & 2.15 & 2400 & 0.56 & 3.69 \\
\hline 500 & 0.12 & 0.77 & 1500 & 0.35 & 2.30 & 2500 & 0.59 & 3.84 \\
\hline 600 & 0.14 & 0.92 & 1600 & 0.38 & 2.46 & 2600 & 0.61 & 3.99 \\
\hline 700 & 0.16 & 1.08 & 1700 & 0.40 & 2.61 & 2700 & 0.63 & 4.15 \\
\hline 800 & 0.19 & 1.23 & 1800 & 0.42 & 2.77 & 2800 & 0.66 & 4.30 \\
\hline 900 & 0.21 & 1.38 & 1900 & 0.45 & 2.92 & 2900 & 0.68 & 4.46 \\
\hline 1000 & 0.24 & 1.54 & 2000 & 0.47 & 3.07 & 3000 & 0.71 & 4.61 \\
\hline
\end{tabular}

Table 7.1 introduces the question, "Since the air flow velocity out of the frit pores is a factor of $\sim 6.5(1 / 0.153)$ higher than the superficial velocity, and the air flow velocity returns to the superficial velocity at some height $\left(\mathrm{h}_{\mathrm{s}}\right)$ within the sample boat above the frit, how does the air flow behave between the frit and the height $h_{s}$ ?" There is no way to know the answer to this question completely. However, a brief conceptualization of the problem will lead to a better understanding and a way in which conservative bounds can be implemented to produce results for this problem. As the air flows rapidly from the jets created by frit pores, the air just above the frit between the pores remains relatively stagnant. This would be the case if there were no powder particles present anyway. As the air jets emerge from the pore, they immediately interact with the edges of the stagnant air pockets, causing portions of the air pockets to move upward and creating a drag on the edges of the jets. This phenomenon leads to mixing and a dispersion of flow momentum across the flow area of the sample boat. This convective mixing is a much faster mixing mechanism than the diffusion used in the calculation in Eq. (7.3), which reinforces the claim that the result of Eq. (7.3) is conservative. Since the actual vertical flow rate of the suspending air between the frit and the height $h_{s}$ at which superficial flow is re-achieved is complex, it is conservative to assume that the air-flow velocity in this region is constant at the post-frit velocity of Table 7.1 and that the air flow velocity above $h_{s}$ is equal to the superficial velocity. Thus, the system to be analyzed is as appears in Figure 7.1. 


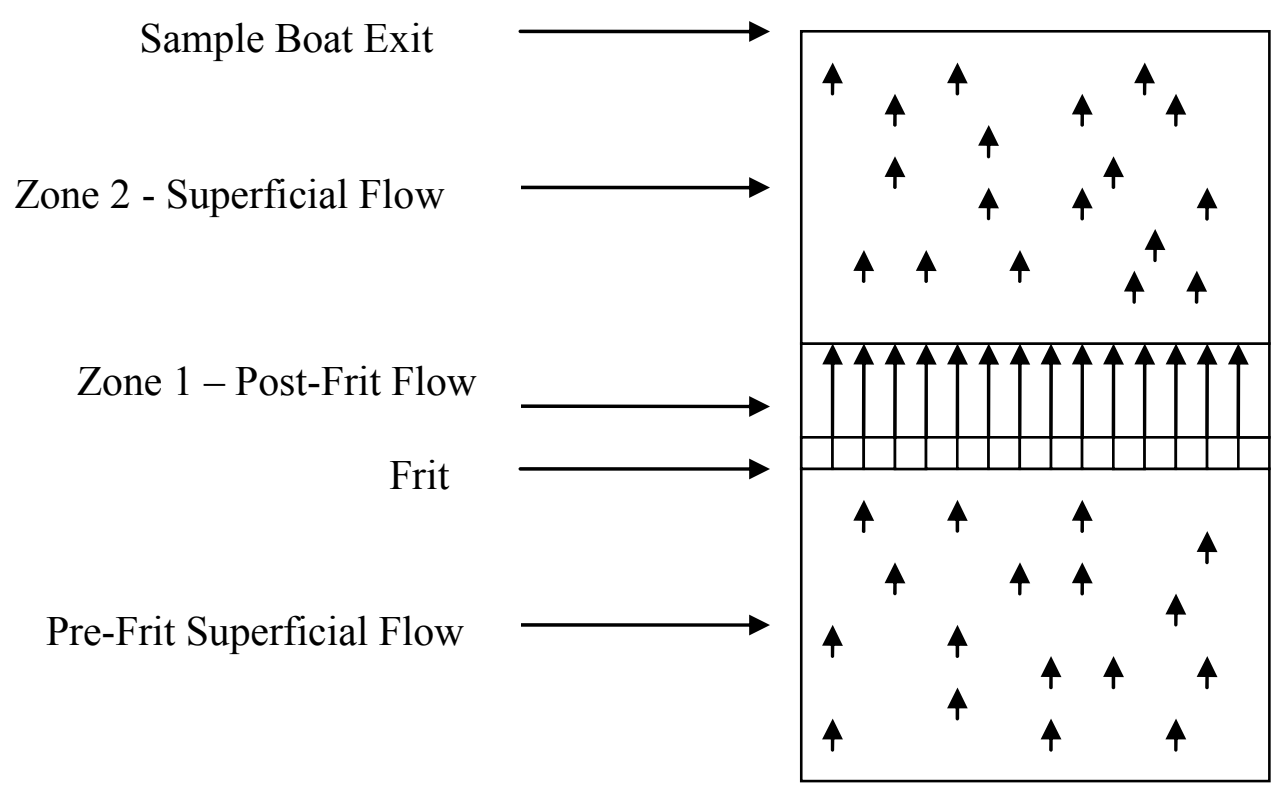

Figure 7.1. Flow System Being Modeled in this Section

\subsection{Force Balances and Trajectory Calculations}

The first suspending air flow that particles are subjected to is the series of air jets emerging from the frit. To make sure that particles do not fall down into the frit pores, the radius of a given frit pore must necessarily be smaller than the particle located above it. In the case that the particle diameter is smaller than the pore diameter, the upward air flow will completely engulf the particle, leading to another force balance to be discussed later. For the case that the particles are larger than 0.5 microns, the jets impinging on the bottom of the particles are smaller than the particles themselves. The forces acting on a given particle in this case are as displayed in Figure 7.2. Here, the notation is as follows:

- $\mathrm{F}_{\text {con }}$ - the upward convective force on the particle due to the impinging jets below the particle

- $\mathrm{F}_{\text {buo }}$ - the buoyant force of the air in the jet below the particle

- $\mathrm{F}_{\mathrm{D}}$ - the drag force imparted on the particle by the still air that it is being pushed through by the jet

- $\mathrm{F}_{\mathrm{G}}$ - the gravitational force on the particle

- $\mathrm{n}$ - the number of jets impinging on the particle of interest

- $v_{1}$ - the velocity of the air jet

- $\mathrm{R}_{1}$ - the radius of the air jet

- $\rho_{\mathrm{g}}$ - the air density

- $\mathrm{g}$ - the acceleration due to gravity

- $\mathrm{h}$ - the height of the particle above the frit

- $\mathrm{t}$ - the time after the initialization of air flow 
- $\eta$ - the air viscosity

- $\mathrm{d}_{\mathrm{p}}$ - the particle diameter

- $\mathrm{m}_{\mathrm{p}}$-the particle mass

- $\mathrm{V}$ - the particle velocity taken to be positive in the upward direction.

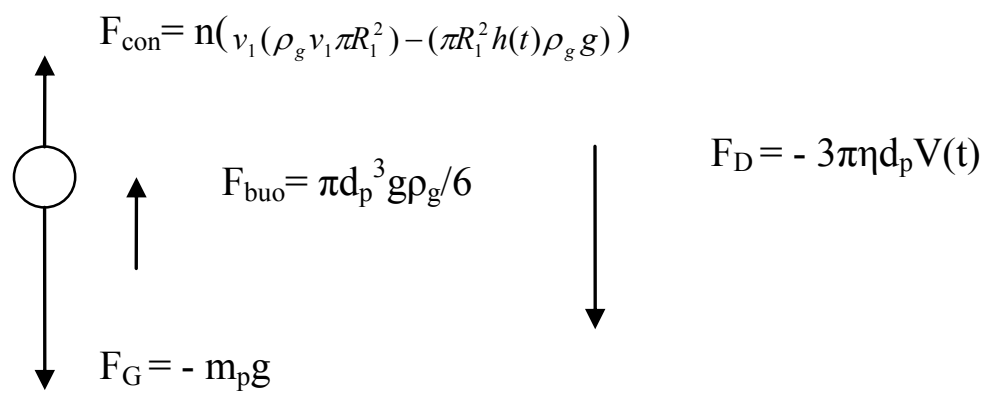

Figure 7.2. Post-Frit (Zone 1) Force Balance on a Particle

The scenario in Figure 7.2 can be evaluated for the case of any number of jets impinging upon the bottom of a particle as long as the total area of all jets combined does not exceed the impact area of the particle. However, calculating the trajectory of each particle size for each impinging jet scenario is an exhaustive exercise. Instead, it is better to find a bounding case. The greatest upward force that can possibly be imparted upon a particle due to an upward air flow is that which occurs when the particle is completely engulfed in the upward air stream. Figure 7.3 illustrates this condition.

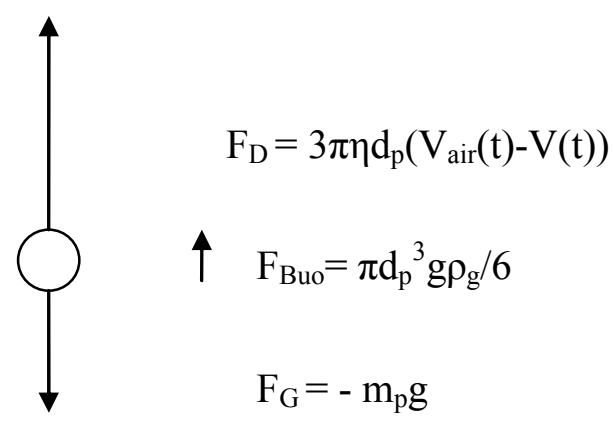

Figure 7.3. Force Balance on a Particle Engulfed by an Upward Air Stream

The force balance in Figure 7.2 is simpler than the one in Figure 7.3. In Figure 7.3, the drag force is in the upward direction because the air is flowing past the particle at a higher velocity than the particle is moving. The upward convective force in Figure 7.2 becomes part of the upward drag force in Figure 7.3. The force balance in Figure 7.3, however, is more complicated than that in Figure 7.2 in one aspect. It makes it necessary to know the air-flow velocity as a function of time. Since this is not known, it is necessary to make conservative estimations. This is what we have actually already done by dividing the air flow up into two zones as shown in Figure 7.1. In the future momentum analyses performed in this section, the conservative force 
balance shown in Figure 7.3 will be used where the air-flow velocity below the height $h_{\mathrm{s}}$ will be treated as a constant equal to the post-frit air jet velocity, and the air flow velocity above the height $h_{s}$ will be treated as a constant equal to the superficial air-flow velocity.

The force balance shown in Figure 7.3 can be written as

$$
m_{p} \frac{d V(t)}{d t}=3 \pi \eta d_{p}\left(V_{a i r}(t)-V(t)\right)-\left(\rho_{p}-\rho_{g}\right) \frac{\pi d_{p}^{3} g}{6}
$$

which can be integrated to give

$$
V_{1}(t)=V_{\mathrm{air}, 1}-\frac{\left(3 \pi \eta d_{p} V_{\mathrm{air}, 1}-\left(\rho_{p}-\rho_{g}\right) \frac{\pi d_{p}^{3} g}{6}\right) e^{-\frac{3 \pi \eta d_{p} t}{m_{p}}}+\left(\rho_{p}-\rho_{g}\right) \frac{\pi d_{p}^{3} g}{6}}{3 \pi \eta d_{p}}
$$

where $V_{1}(t)$ is the velocity of the particle within Zone 1 of Figure 7.1. Likewise, $V_{\text {air, } 1}$ is the velocity of the air within Zone 1 of Figure 7.1. The reason that $\mathrm{V}_{1}(\mathrm{t})$ must be specified as such is that a boundary condition is necessary in order to produce Eq. (7.7). The boundary condition that was used was that the velocity at $t=0$ is 0 . Also, $V_{\text {air, } 1}$ must be specified because future equations involving the air velocity in Zone 2 of Figure 7.1 will be specified as $V_{\text {air,2. }}$.

As written, Eq. (7.7) is a function of the time elapsed after the initiation of air flow. However, the time at which the particle reaches Zone 2 is not known. Instead, it is the height above the frit at which Zone 2 begins that is known. To determine the height of the particle as a function of time, Eq. (7.7) can be integrated over time to give

$$
\begin{aligned}
& h_{1}(t)=\frac{m_{p}}{3 \pi \eta d_{p}}\left(V_{\mathrm{air}, 1}-\frac{\left(\rho_{p}-\rho_{g}\right) \frac{\pi d_{p}^{3} g}{6}}{3 \pi \eta d_{p}}\right) e^{-\frac{3 \pi \eta d_{p} t}{m_{p}}}+V_{\mathrm{air}, 1} t \\
& +\frac{m_{p}}{3 \pi d_{p}}\left(-\left(\rho_{p}-\rho_{g}\right) \frac{\pi d_{p}^{3} g}{6 m_{p}} t-V_{\mathrm{air}, 1}+\frac{\left(\rho_{p}-\rho_{g}\right) \frac{\pi d_{p}^{3} g}{6}}{3 \pi \eta d_{p}}\right)
\end{aligned}
$$

Now, for a given system, $h_{1}(t)$ can be set equal to $h_{s}$ and Eq. (7.8) solved for $t$. This value of t can be substituted into Eq. (7.7) to determine the velocity of a particle once it reaches Zone 2. This velocity can be used as the initial condition to solve Eq. (7.6) in Zone 2. Likewise, $\mathrm{h}_{\mathrm{s}}$ can be used as an initial condition in the resulting equation to solve for the height of the particle as a function of time in Zone 2. In doing so, it is determined that in Zone 2, 


$$
V_{2}(t)=V_{\mathrm{air}, 2}-\frac{\left(\rho_{p}-\rho_{g}\right) d_{p}^{2} g}{18 \eta}-\frac{\left(18 \eta V_{\mathrm{air}, 2}-\left(\rho_{p}-\rho_{g}\right) d_{p}^{2} g-18 \eta V_{1}\left(t_{h_{s}}\right)\right) e^{-\frac{3 \pi \eta d_{p}\left(t-t_{h_{s}}\right)}{m_{p}}}}{18 \eta}
$$

where $V_{1}\left(t_{h}\right)$ is the velocity achieved by the particle by the time it reaches a height of $h_{s}$ and

$$
\begin{aligned}
& h_{2}(t)=h_{s}+V_{\text {air }, 2}\left(t-t_{h_{s}}\right)+\frac{d_{p}^{2} g\left(\rho_{p}-\rho_{g}\right)}{18 \eta}\left(t_{h_{s}}-t\right)+\frac{d_{p} m_{p} g\left(\rho_{p}-\rho_{g}\right)}{54 \eta^{2} \pi} \\
& +\frac{m_{p}}{3 d_{p} \eta \pi}\left(V\left(t_{h_{s}}\right)-V_{\text {air }, 2}\right)-\frac{d_{p} g m_{p}\left(\rho_{p}-\rho_{g}\right) e^{-\frac{3 d_{p} \eta \pi}{m_{p}}\left(t-t_{h_{s}}\right)}}{54 \eta^{2} \pi} \\
& +\frac{m_{p} e^{-\frac{3 d_{p} \eta \pi}{m_{p}}\left(t-t_{h_{s}}\right)}}{3 \pi d_{p} \eta}\left(V_{\text {air }, 2}-V\left(t_{h_{s}}\right)\right)
\end{aligned}
$$

\subsection{Parametric Studies}

Equations (7.7) to (7.10) are complicated, but in conjunction with Eq. (7.6), they can readily be used to generate particle trajectories through the flow system for various volumetric flow rates. Essentially, these trajectory calculations can be used to determine critical parameter values for each flow case. These critical parameters are 1) the largest particle size that can be suspended by the post-frit flow in Zone 1 and 2) the largest particle size that can be suspended by the superficial flow in Zone 2. Theoretically, all of the particles that are suspended by the flow velocity in Zone 2 will be carried from the sample boat. However, since the velocity of the flow in Zone 2 is significantly lower than the velocity of the flow in Zone 1, there will be a size range for which particles will be projected from Zone 1 into Zone 2 only to fall back to the boundary between the two zones. How high each of the particles in this range travel into Zone 2 before falling back to the boundary will be determined by the particle size and the velocity that the particle achieves before crossing the boundary. There is a possibility that particles that are not suspended by the flow in Zone 2 will gain enough momentum by the time they reach the zone boundary to be carried from the boat. The purpose of the parametric studies in this section is to determine the size ranges corresponding to each of these phenomena for each flow scenario.

The results presented in Table 7.2 list the particle sizes that would be suspended in Zone 1 by the air jets generated by a given flow rate. These values are determined by setting the right-hand side of Eq. (7.6) for $t=0$ equal to zero and solving for the corresponding value of $d_{p}$. The results in Table 7.3 list the critical size for which a particle will always have an upward velocity in Zone 2. Particles with diameters larger than those listed in Table 7.3 will decelerate with time, and their vertical velocities will eventually become negative, causing them to fall back to the zone boundary. The values in Table 7.3 are determined by evaluating Eq. (7.9) for $t=\infty$. For this reason, the parameter values are labeled $V_{2}(\infty)$. Mathematically, these values represent the largest particle size for which the particle velocity will retain a positive value for all time values greater than 0 for a sustained flow. Thus, the particle-size values in Table 7.3 should be 
interpreted as the critical size value for which all smaller particles, if suspended into Zone 2, will definitely be lifted from the sample boat if they do not agglomerate or stick to the sample boat wall.

Table 7.2. Maximum Particle Size with an Initial Upward Acceleration

\begin{tabular}{|c|c|c|c|c|c|c|c|c||}
\hline $\begin{array}{c}\text { Volumetric } \\
\text { Flow Rate } \\
\text { SCCM }\end{array}$ & $\begin{array}{c}\text { Post- } \\
\text { Frit } \\
\text { Velocity } \\
\mathbf{m} / \mathbf{s}\end{array}$ & $\begin{array}{c}\text { Max. Sus. } \\
\text { Part. Size } \\
\boldsymbol{\mu m}\end{array}$ & $\begin{array}{c}\text { Volumetric } \\
\text { Flow Rate } \\
\text { SCCM }\end{array}$ & $\begin{array}{c}\text { Post- } \\
\text { Frit } \\
\text { Velocity } \\
\mathbf{m} / \mathbf{s}\end{array}$ & $\begin{array}{c}\text { Max. Sus. } \\
\text { Part. Size } \\
\boldsymbol{\mu m}\end{array}$ & $\begin{array}{c}\text { Volumetric } \\
\text { Flow Rate } \\
\text { SCCM }\end{array}$ & $\begin{array}{c}\text { Post- } \\
\text { Frit } \\
\text { Velocity } \\
\mathbf{m} / \mathbf{s}\end{array}$ & $\begin{array}{c}\text { Max. Sus. } \\
\text { Part. Size } \\
\boldsymbol{\mu m}\end{array}$ \\
\hline 100 & 0.15 & 25.07 & 1100 & 1.69 & 83.16 & 2100 & 3.23 & 114.90 \\
\hline 200 & 0.31 & 35.46 & 1200 & 1.84 & 86.86 & 2200 & 3.38 & 117.61 \\
\hline 300 & 0.46 & 43.43 & 1300 & 2.00 & 90.41 & 2300 & 3.53 & 120.25 \\
\hline 400 & 0.61 & 50.15 & 1400 & 2.15 & 93.82 & 2400 & 3.69 & 122.84 \\
\hline 500 & 0.77 & 56.07 & 1500 & 2.30 & 97.11 & 2500 & 3.84 & 125.37 \\
\hline 600 & 0.92 & 61.42 & 1600 & 2.46 & 100.30 & 2600 & 3.99 & 127.85 \\
\hline 700 & 1.08 & 66.34 & 1700 & 2.61 & 103.38 & 2700 & 4.15 & 130.29 \\
\hline 800 & 1.23 & 70.92 & 1800 & 2.77 & 106.38 & 2800 & 4.30 & 132.68 \\
\hline 900 & 1.38 & 75.22 & 1900 & 2.92 & 109.29 & 2900 & 4.46 & 135.03 \\
\hline 1000 & 1.54 & 79.29 & 2000 & 3.07 & 112.13 & 3000 & 4.61 & 137.34 \\
\hline
\end{tabular}

Comparison of the data in Table 7.2 and Table 7.3 supports the assertion that for each flow rate, there is a particle-size range in which it is possible that the particles suspended from Zone 1 may not be carried completely from the boat because of the insufficient upward drag force provided by the air flow in Zone 2. To determine which particles in this range are carried out of the sample boat and which are not, it is necessary to solve Eqs. (7.7) to (7.10) to determine the complete trajectory of a given particle through the volume encapsulated by the sample boat. If for a given particle size, $\mathrm{V}_{2}(\infty)<0$, then the time at which the particle velocity is zero will correspond to the time at which the particle reaches its maximum height. If this height is above the height of the boat, then technically the particle should be lifted from the boat. There is a possibility that the particle will fall back into the boat, but it can be conservatively asserted that if the particle is suspended above the height of the boat, then it is officially removed by the air flow. Determining the trajectory of each particle size through the system at each flow rate generates a large amount of data. To condense these data, the trajectory of a particle with a size just larger than the maximum particle size for which $\mathrm{V}_{2}(\infty)>0$, as appears in Table 7.3, is calculated. For example, the trajectory of a 10-micrometer particle at a volumetric flow rate of 100 SCCM will be calculated. The result of this calculation for each flow rate in Table 7.3 is shown in Table 7.4.

Table 7.4 shows that the peak trajectory height for particles only a bit larger than those for which $\mathrm{V}_{2}(\infty)>0$ is well beneath the 3.81-cm height of the sample boat. Particles larger than those considered in Table 7.4 will have lower maximum trajectory heights. For instance, according to Table 7.2, the maximum particle size that will be suspended by the post-frit velocity for a 100SCCM flow rate is 25.07 micrometers, and according to Table 7.3, all particles less than 9.81 micrometers can be expected to be lifted completely from the sample boat. Table 7.4 asserts that a 10-micrometer particle subjected to a 100-SCCM flow rate within the test apparatus will reach a maximum trajectory height of $0.003 \mathrm{~cm}$, which is much less than the $3.81-\mathrm{cm}$ height of the sample boat. All larger particles will have a lower maximum trajectory. To support this 
assertion, Table 7.5 shows the maximum trajectory height for particle sizes between 10 micrometers and 25 micrometers for the 100-SCCM case.

Table 7.3. Maximum Particle Size for which the Final Particle Velocity is Greater than 0 for each Volumetric Flow Rate

\begin{tabular}{|c|c|c|c|c|c||}
\hline $\begin{array}{c}\text { Molumetric } \\
\text { Flow Rate } \\
\text { SCCM }\end{array}$ & $\begin{array}{c}\text { Marticle Size } \\
\text { for which } \\
\mathbf{V}_{\mathbf{2}}(\boldsymbol{\infty})>\mathbf{0} \\
\boldsymbol{\mu m}\end{array}$ & $\begin{array}{c}\text { Volumetric } \\
\text { Flow Rate } \\
\text { SCCM }\end{array}$ & $\begin{array}{c}\text { Max. } \\
\text { Particle Size } \\
\text { for which } \\
\mathbf{V}_{\mathbf{2}}(\boldsymbol{\infty})>\mathbf{0} \\
\boldsymbol{\mu m}\end{array}$ & $\begin{array}{c}\text { Max. } \\
\text { Volumetric } \\
\text { Flow Rate } \\
\text { SCCM }\end{array}$ & $\begin{array}{c}\text { Particle Size } \\
\text { for which } \\
\mathbf{V}_{\mathbf{2}}(\boldsymbol{\infty})>\mathbf{0} \\
\boldsymbol{\mu m}\end{array}$ \\
\hline 100 & 9.81 & 1100 & 32.53 & 2100 & 44.94 \\
\hline 200 & 13.87 & 1200 & 33.98 & 2200 & 46.00 \\
\hline 300 & 16.99 & 1300 & 35.36 & 2300 & 47.04 \\
\hline 400 & 19.62 & 1400 & 36.70 & 2400 & 48.05 \\
\hline 500 & 21.93 & 1500 & 37.99 & 2500 & 49.04 \\
\hline 600 & 24.02 & 1600 & 39.23 & 2600 & 50.01 \\
\hline 700 & 25.95 & 1700 & 40.44 & 2700 & 50.96 \\
\hline 800 & 27.74 & 1800 & 41.61 & 2800 & 51.90 \\
\hline 900 & 29.42 & 1900 & 42.75 & 2900 & 52.82 \\
\hline 1000 & 31.01 & 2000 & 43.86 & 3000 & 53.72 \\
\hline
\end{tabular}

The maximum height reached by particles in other flow rates matches the trend shown for a flow rate of 100 SCCM in Table 7.5. The result of the analysis above is that under the assumptions made thus far, the presence of the frit makes no significant impact on the PSD leaving the sample boat for each test flow rate. As mentioned earlier, however, the analysis was based on a two-zone approach in which the height of the separation between the two zones was determined through an order-of-magnitude estimation. Further analysis is necessary to quantify the effect that this estimation has on the resulting particle trajectories. The most transparent parametric study in this case is to determine the maximum particle size expected to be fully suspended from the boat if $h_{s}$ is taken to be higher than it was in the previous analysis.

This parametric study will start with the case of a 100-SCCM flow rate. The air-speed velocity does not change in Zone 1 or Zone 2. Thus, the results in Table 7.2 and Table 7.3 are still applicable. Even if the value of $h_{s}$ is changed, it is still the case that for a 100-SCCM flow rate, all particles less than 9.81 micrometers can be expected to be released if they are entrained in the flow, and only particles smaller than 25.07 micrometers will be lifted from the frit because of the post-frit air velocity. However, for a higher value of $h_{s}$, it is possible that some fraction of the particle between 9.81 micrometers and 25 micrometers may be provided enough momentum to be suspended from the sample boat. When this phenomenon is investigated, it is determined that $h_{s}$ must have a value of $3.78 \mathrm{~cm}$ before a 10 -micrometer particle will be lifted from the sample boat for a flow rate of 100 SCCM. The reason for this result has to do with the overwhelming effect of the drag force on particles of the size range being considered in this study. Once the particle reaches zone 2 , it slows down very rapidly. 
Table 7.4. Particle Trajectory Parameter Values

\begin{tabular}{|c|c|c|c|c|}
\hline $\begin{array}{c}\text { Flow Rate } \\
\text { SCCM }\end{array}$ & $\begin{array}{c}\mathbf{h}_{\mathrm{s}} \\
\boldsymbol{\mu m}\end{array}$ & $\begin{array}{l}\mathrm{dp} \\
\mu \mathrm{m}\end{array}$ & $\begin{array}{c}\mathbf{V 1}\left(\mathbf{t}_{\mathbf{h}_{\mathrm{s}}}\right) \\
\mathbf{m} / \mathbf{s}\end{array}$ & $\begin{array}{c}\text { Max Height } \\
\text { cm }\end{array}$ \\
\hline 100 & 1.54 & 10 & 0.012 & 0.00259 \\
\hline 200 & 3.07 & 14 & 0.018 & 0.00761 \\
\hline 300 & 4.61 & 17 & 0.022 & 0.01586 \\
\hline 400 & 6.15 & 20 & 0.025 & 0.01782 \\
\hline 500 & 7.68 & 22 & 0.028 & 0.03168 \\
\hline 600 & 9.22 & 25 & 0.030 & 0.02426 \\
\hline 700 & 10.75 & 26 & 0.034 & 0.05359 \\
\hline 800 & 12.29 & 28 & 0.036 & 0.05442 \\
\hline 900 & 13.83 & 30 & 0.037 & 0.05341 \\
\hline 1000 & 15.36 & 32 & 0.039 & 0.05180 \\
\hline 1100 & 16.90 & 33 & 0.042 & 0.07645 \\
\hline 1200 & 18.44 & 34 & 0.044 & 0.12372 \\
\hline 1300 & 19.97 & 36 & 0.045 & 0.08977 \\
\hline 1400 & 21.51 & 37 & 0.047 & 0.12173 \\
\hline 1500 & 23.05 & 38 & 0.050 & 0.17544 \\
\hline 1600 & 24.58 & 40 & 0.050 & 0.11475 \\
\hline 1700 & 26.12 & 41 & 0.052 & 0.13923 \\
\hline 1800 & 27.66 & 42 & 0.054 & 0.16774 \\
\hline 1900 & 29.19 & 43 & 0.055 & 0.19970 \\
\hline 2000 & 30.73 & 44 & 0.057 & 0.23550 \\
\hline 2100 & 32.26 & 45 & 0.059 & 0.27569 \\
\hline 2200 & 33.80 & 47 & 0.059 & 0.16686 \\
\hline 2300 & 35.34 & 48 & 0.060 & 0.18073 \\
\hline 2400 & 36.87 & 49 & 0.061 & 0.19379 \\
\hline 2500 & 38.41 & 50 & 0.063 & 0.20503 \\
\hline 2600 & 39.95 & 51 & 0.062 & 0.20762 \\
\hline 2700 & 41.48 & 51 & 0.067 & 0.41034 \\
\hline 2800 & 43.02 & 52 & 0.068 & 0.40319 \\
\hline 2900 & 44.56 & 53 & 0.069 & 0.39561 \\
\hline 3000 & 46.09 & 54 & 0.070 & 0.38654 \\
\hline
\end{tabular}


Table 7.5. Maximum Vertical Height Achieved by Particles in a 100-SCCM Flow

\begin{tabular}{|c|c|}
\hline $\begin{array}{c}\text { dp } \\
\boldsymbol{\mu m}\end{array}$ & $\begin{array}{c}\text { Max. Height } \\
\boldsymbol{\mu m}\end{array}$ \\
\hline 10 & 25.88 \\
\hline 11 & 15.64 \\
\hline 12 & 11.17 \\
\hline 13 & 8.52 \\
\hline 14 & 6.77 \\
\hline 15 & 5.53 \\
\hline 16 & 4.62 \\
\hline 17 & 3.93 \\
\hline 18 & 3.39 \\
\hline 19 & 2.96 \\
\hline 20 & 2.60 \\
\hline 21 & 2.31 \\
\hline 22 & 2.07 \\
\hline 23 & 1.87 \\
\hline 24 & 1.69 \\
\hline 25 & 1.55 \\
\hline
\end{tabular}

It has been assumed throughout this chapter that the drag force on the particle is governed by the Stokes equation. However, for this to be a reasonable assumption, the particle Reynolds number must be less than or equal to 1 . For several of the particles in this section, the particle Reynolds number is greater than 1 . Thus, it is important to determine which particles have Reynolds numbers causing their motion to be outside of the range for application of the Stokes equation and how their behavior can be expected to deviate from the results generated by the models described in this chapter.

The particle Reynolds number is defined as

$$
\operatorname{Re}_{p}=\frac{d_{p} V \rho_{g}}{\eta}
$$

where $V$ is the relative velocity between the particle and the air. In the analyses presented in this chapter, the density and the viscosity of the air are constant, so the only two variables in Eq. (7.11) are the relative particle velocity and the particle diameter. Since the gas density is 1.2 $\mathrm{kg} / \mathrm{m}^{3}$ and the gas viscosity is $18.6 \times 10^{-6} \mathrm{~Pa} \mathrm{~s}$, the product of the particle diameter and the relative particle velocity must be less than $1.5 \times 10^{-5} \mathrm{~m}^{2} / \mathrm{s}$ for the particle Reynolds number to be less than or equal to 1 . This is not the case for some particle sizes and flow rates as seen in Table 7.1. Since it can be seen that the Stokes Equation is not valid for some of the cases analyzed in this section, it must be demonstrated that the use of the Stokes equation to describe them is conservative. 
The reason that the Reynolds number is used to determine whether the Stokes Equation is applicable is that it represents the ratio of the particle inertia to the viscous force acting on the particle due to the surrounding fluid. Thus, for very small particle Reynolds numbers, the particle motion is dominated by the drag applied by the surrounding fluid, and for very large particle Reynolds numbers, the particle motion is dominated by the particle's inertia. Thus, the Stokes Equation is a limit in which the particle inertia is neglected and the viscous drag force is dominant. As can be seen in Figure 7.3, the drag force for the cases being considered here is in the upward direction, so the conservative equation is the equation that maximizes this force. Thus, the Stokes Equation is the conservative equation in the cases where the drag force is accelerating the particle. However, when considering the slowing down of the particle in Zone 2, the drag force is acting against the particle upward motion and is not conservative. So, the Stokes Equation can be conservatively used as the particle moves to $h_{s}$, but may not be appropriate for describing particle motion above $h_{s}$. For this reason, careful attention must be paid to the particle Reynolds number once the particle reaches Zone 2.

Table 7.6 shows the effect of increasing the value of $h_{s}$ in the 100-SCCM case on particle release for the bounding particle sizes shown in Table 7.3 and Table 7.4. Table 7.6 verifies the claim made earlier that the value of $h_{s}$ must be nearly equal to the height of the sample boat for even 10-micrometer particles to receive enough extra momentum from the post-frit velocity to be expelled from the sample boat. The sample boat is 1.5 inches or $3.81 \mathrm{~cm}$ tall. Notice how even if $\mathrm{h}_{\mathrm{s}}$ is taken to be $3.8 \mathrm{~cm}, 25$-micrometer particles will not be lifted from the sample boat. An analysis of Table 7.6 shows that there is a value of $h_{s}$ for which the velocity of the particle at $h_{s}$ is maximized. This occurs at or before $h_{s}=0.01 \mathrm{~cm}$ for 25 -micrometer particles and at or before $h_{s}$ $=1 \mathrm{~cm}$ for 10-micrometer particles. Since there is a maximum velocity that a given particle can achieve from acceleration in Zone 1, there is a maximum distance that a given particle will travel above $h_{s}$ because of extra imparted momentum in Zone 1. Inspection of column 4 of Table 7.6 shows that for a flow rate of $100 \mathrm{SCCM}$, the maximum distance that a 10 -micrometer particle will travel in the test system because of excess imparted momentum is $3.11 \times 10^{-2} \mathrm{~cm}$. This distance for a 25 -micrometer particle is $5 \times 10^{-6} \mathrm{~cm}$. Thus, if $\mathrm{h}_{\mathrm{s}}$ is considered to be equal to 3.779 $\mathrm{cm}$, then 10-micrometer particles can theoretically be expected to be lifted from the sample boat. The value of $h_{s}$ must be taken to be larger for excess momentum to influence the release of particles greater than 10 micrometers. The particle Reynolds number for each of the cases considered in Table 7.6 is less than 1, and thus the results presented can be considered accurate.

Table 7.7 investigates the effect of raising the value of $h_{s}$ for a system volumetric flow rate of 200 SCCM. In Table 7.7, 14-micrometer and 15-micrometer particles are compared to quantize more closely the effect of particle size. Table 7.7 shows that in order for excess momentum to drive out any particles larger than the particles expected to be driven out by a superficial flow velocity, the value of $h_{s}$ must again be nearly equal to the height of the boat. The interesting feature presented in Table 7.7 is that if the value of $h_{s}$ is greater than roughly $1 \mathrm{~cm}, 15$ micrometer particles will be pushed slightly higher than 14-micrometer particles. This phenomenon is due to the fact that at this height, the particles will have to be accelerated for enough time to have obtained enough momentum to be propelled significantly into Zone 2. Still, for excess momentum to be of any consequence, the value of $h_{s}$ must be orders of magnitude above that predicted by Eq. (7.3). The particle Reynolds numbers presented in Table 7.7 indicate that the cases explored are still accurately characterized by the equations of Stokes flow. 
Table 7.6. Adjustment of $h_{s}$ Value for a 100-SCCM Flowrate

\begin{tabular}{|c|c|c|c|c||}
\hline $\begin{array}{c}\mathbf{d}_{\mathbf{p}} \\
\boldsymbol{\mu m}\end{array}$ & $\begin{array}{c}\mathbf{h}_{\mathbf{s}} \\
\mathbf{c m}\end{array}$ & $\begin{array}{c}\mathbf{V}_{\mathbf{1}}\left(\mathbf{t}_{\mathbf{s}}\right) \\
\mathbf{m} / \mathbf{s}\end{array}$ & $\begin{array}{c}\text { Max. Height } \\
\mathbf{c m}\end{array}$ & $\mathbf{R e}_{\mathbf{p}}$ \\
\hline 10 & $3.0 \mathrm{E}-04$ & $1.68 \mathrm{E}-02$ & $3.813955 \mathrm{E}-03$ & $4.30 \mathrm{E}-03$ \\
\hline 25 & $3.0 \mathrm{E}-04$ & $4.70 \mathrm{E}-04$ & $3.013895 \mathrm{E}-04$ & $3.72 \mathrm{E}-02$ \\
\hline 10 & $1.0 \mathrm{E}-03$ & $2.96 \mathrm{E}-02$ & $7.565767 \mathrm{E}-03$ & $3.91 \mathrm{E}-03$ \\
\hline 25 & $1.0 \mathrm{E}-03$ & $6.99 \mathrm{E}-04$ & $1.002985 \mathrm{E}-03$ & $3.68 \mathrm{E}-02$ \\
\hline 10 & $1.0 \mathrm{E}-02$ & $7.70 \mathrm{E}-02$ & $2.817759 \mathrm{E}-02$ & $3.45 \mathrm{E}-02$ \\
\hline 25 & $1.0 \mathrm{E}-02$ & $9.05 \mathrm{E}-04$ & $1.000492 \mathrm{E}-02$ & $3.65 \mathrm{E}-02$ \\
\hline 10 & $1.0 \mathrm{E}-01$ & $1.27 \mathrm{E}-01$ & $1.305398 \mathrm{E}-01$ & $6.68 \mathrm{E}-02$ \\
\hline 25 & $1.0 \mathrm{E}-01$ & $9.05 \mathrm{E}-04$ & $1.000050 \mathrm{E}-01$ & $3.65 \mathrm{E}-02$ \\
\hline 10 & $1.0 \mathrm{E}+00$ & $1.29 \mathrm{E}-01$ & $1.031077 \mathrm{E}+00$ & $6.82 \mathrm{E}-02$ \\
\hline 25 & $1.0 \mathrm{E}+00$ & $9.05 \mathrm{E}-04$ & $1.000005 \mathrm{E}+00$ & $3.65 \mathrm{E}-02$ \\
\hline 10 & $2.0 \mathrm{E}+00$ & $1.29 \mathrm{E}-01$ & $2.031077 \mathrm{E}+00$ & $6.82 \mathrm{E}-02$ \\
\hline 25 & $2.0 \mathrm{E}+00$ & $9.05 \mathrm{E}-04$ & $2.000005 \mathrm{E}+00$ & $3.65 \mathrm{E}-02$ \\
\hline 10 & $3.0 \mathrm{E}+00$ & $1.29 \mathrm{E}-01$ & $3.031077 \mathrm{E}+00$ & $6.82 \mathrm{E}-02$ \\
\hline 25 & $3.0 \mathrm{E}+00$ & $9.05 \mathrm{E}-04$ & $3.000005 \mathrm{E}+00$ & $3.65 \mathrm{E}-02$ \\
\hline 10 & $3.8 \mathrm{E}+00$ & $1.29 \mathrm{E}-01$ & $3.831077 \mathrm{E}+00$ & $6.82 \mathrm{E}-02$ \\
\hline 25 & $3.8 \mathrm{E}+00$ & $9.05 \mathrm{E}-04$ & $3.800005 \mathrm{E}+00$ & $3.65 \mathrm{E}-02$ \\
\hline
\end{tabular}

Table 7.7. Adjustment of $h_{s}$ Value for a 200-SCCM Flowrate

\begin{tabular}{|c|c|c|c|c||}
\hline $\begin{array}{c}\mathbf{d}_{\mathbf{p}} \\
\boldsymbol{\mu m}\end{array}$ & $\begin{array}{c}\mathbf{h}_{\mathbf{s}} \\
\mathbf{c m}\end{array}$ & $\begin{array}{c}\mathbf{V}_{\mathbf{1}}\left(\mathbf{t}_{\mathbf{s}}\right) \\
\mathbf{m} / \mathbf{s}\end{array}$ & $\begin{array}{c}\text { Max. Height } \\
\mathbf{c m}\end{array}$ & $\mathbf{R e}_{\mathbf{p}}$ \\
\hline 14 & $3.0 \mathrm{E}-04$ & $1.68 \mathrm{E}-02$ & $7.514022 \mathrm{E}-03$ & $2.67 \mathrm{E}-02$ \\
\hline 15 & $3.0 \mathrm{E}-04$ & $4.70 \mathrm{E}-04$ & $4.379895 \mathrm{E}-03$ & $2.99 \mathrm{E}-02$ \\
\hline 14 & $1.0 \mathrm{E}-03$ & $2.96 \mathrm{E}-02$ & $1.471237 \mathrm{E}-02$ & $1.43 \mathrm{E}-02$ \\
\hline 15 & $1.0 \mathrm{E}-03$ & $6.99 \mathrm{E}-04$ & $1.032740 \mathrm{E}-02$ & $1.76 \mathrm{E}-02$ \\
\hline 14 & $1.0 \mathrm{E}-02$ & $7.70 \mathrm{E}-02$ & $5.192302 \mathrm{E}-02$ & $3.87 \mathrm{E}-02$ \\
\hline 15 & $1.0 \mathrm{E}-02$ & $9.05 \mathrm{E}-04$ & $4.585858 \mathrm{E}-02$ & $3.52 \mathrm{E}-02$ \\
\hline 14 & $1.0 \mathrm{E}-01$ & $1.27 \mathrm{E}-01$ & $1.984102 \mathrm{E}-01$ & $1.44 \mathrm{E}-01$ \\
\hline 15 & $1.0 \mathrm{E}-01$ & $9.05 \mathrm{E}-04$ & $1.948206 \mathrm{E}-01$ & $1.43 \mathrm{E}-01$ \\
\hline 14 & $1.0 \mathrm{E}+00$ & $1.29 \mathrm{E}-01$ & $1.124322 \mathrm{E}+00$ & $1.92 \mathrm{E}-01$ \\
\hline 15 & $1.0 \mathrm{E}+00$ & $9.05 \mathrm{E}-04$ & $1.125944 \mathrm{E}+00$ & $1.99 \mathrm{E}-01$ \\
\hline 14 & $2.0 \mathrm{E}+00$ & $1.29 \mathrm{E}-01$ & $2.124339 \mathrm{E}+00$ & $1.92 \mathrm{E}-01$ \\
\hline 15 & $2.0 \mathrm{E}+00$ & $9.05 \mathrm{E}-04$ & $2.125987 \mathrm{E}+00$ & $1.99 \mathrm{E}-01$ \\
\hline 14 & $3.0 \mathrm{E}+00$ & $1.29 \mathrm{E}-01$ & $3.124339 \mathrm{E}+00$ & $1.92 \mathrm{E}-01$ \\
\hline 15 & $3.0 \mathrm{E}+00$ & $9.05 \mathrm{E}-04$ & $3.125987 \mathrm{E}+00$ & $1.99 \mathrm{E}-01$ \\
\hline 14 & $3.7 \mathrm{E}+00$ & $1.29 \mathrm{E}-01$ & $3.824339 \mathrm{E}+00$ & $1.92 \mathrm{E}-01$ \\
\hline 15 & $3.7 \mathrm{E}+00$ & $9.05 \mathrm{E}-04$ & $3.825987 \mathrm{E}+00$ & $1.99 \mathrm{E}-01$ \\
\hline \hline
\end{tabular}


A calculation of the height traveled by each particle size in each flow rate for various values of $h_{s}$ is a very exhaustive venture. Instead, to conclude the analysis of extra imparted momentum in this section, the maximum height attained by the relevant particle sizes for each flow rate will be determined for a value of $\mathrm{h}_{\mathrm{s}}$ that is 1 order of magnitude larger than that determined from Eq. (3). Table 7.8 through Table 7.18 display these values.

Table 7.8. 100 SCCM

\begin{tabular}{|c|c|c|c|c||}
\hline $\begin{array}{c}\mathbf{d}_{\mathbf{p}} \\
\boldsymbol{\mu m}\end{array}$ & $\begin{array}{c}\mathbf{h}_{\mathbf{s}} \\
\mathbf{c m}\end{array}$ & $\begin{array}{c}\text { Max. } \\
\mathbf{\mathbf { } _ { \mathbf { 1 } }}\left(\mathbf{t}_{\mathbf{s}}\right)\end{array}$ & $\begin{array}{c}\text { Height } \\
\mathbf{c m}\end{array}$ & $\mathbf{R e}_{\mathbf{p}}$ \\
\hline 10 & $1.54 \mathrm{E}-03$ & $3.59 \mathrm{E}-02$ & $9.649 \mathrm{E}-03$ & $8.02 \mathrm{E}-03$ \\
\hline 11 & $1.54 \mathrm{E}-03$ & $3.23 \mathrm{E}-02$ & $7.904 \mathrm{E}-03$ & $6.22 \mathrm{E}-03$ \\
\hline 12 & $1.54 \mathrm{E}-03$ & $2.91 \mathrm{E}-02$ & $6.740 \mathrm{E}-03$ & $4.31 \mathrm{E}-03$ \\
\hline 13 & $1.54 \mathrm{E}-03$ & $2.63 \mathrm{E}-02$ & $5.812 \mathrm{E}-03$ & $2.32 \mathrm{E}-03$ \\
\hline 14 & $1.54 \mathrm{E}-03$ & $2.38 \mathrm{E}-02$ & $5.044 \mathrm{E}-03$ & $2.25 \mathrm{E}-04$ \\
\hline 15 & $1.54 \mathrm{E}-03$ & $2.15 \mathrm{E}-02$ & $4.404 \mathrm{E}-03$ & $1.96 \mathrm{E}-03$ \\
\hline 16 & $1.54 \mathrm{E}-03$ & $1.94 \mathrm{E}-02$ & $3.867 \mathrm{E}-03$ & $4.26 \mathrm{E}-03$ \\
\hline 17 & $1.54 \mathrm{E}-03$ & $1.74 \mathrm{E}-02$ & $3.415 \mathrm{E}-03$ & $6.66 \mathrm{E}-03$ \\
\hline 18 & $1.54 \mathrm{E}-03$ & $1.56 \mathrm{E}-02$ & $3.033 \mathrm{E}-03$ & $9.18 \mathrm{E}-03$ \\
\hline 19 & $1.54 \mathrm{E}-03$ & $1.38 \mathrm{E}-02$ & $2.709 \mathrm{E}-03$ & $1.18 \mathrm{E}-02$ \\
\hline 20 & $1.54 \mathrm{E}-03$ & $1.21 \mathrm{E}-02$ & $2.432 \mathrm{E}-03$ & $1.47 \mathrm{E}-02$ \\
\hline 21 & $1.54 \mathrm{E}-03$ & $1.04 \mathrm{E}-02$ & $2.195 \mathrm{E}-03$ & $1.77 \mathrm{E}-02$ \\
\hline 22 & $1.54 \mathrm{E}-03$ & $8.67 \mathrm{E}-03$ & $1.991 \mathrm{E}-03$ & $2.11 \mathrm{E}-02$ \\
\hline 23 & $1.54 \mathrm{E}-03$ & $6.78 \mathrm{E}-03$ & $1.815 \mathrm{E}-03$ & $2.48 \mathrm{E}-02$ \\
\hline 24 & $1.54 \mathrm{E}-03$ & $4.57 \mathrm{E}-03$ & $1.664 \mathrm{E}-03$ & $2.93 \mathrm{E}-02$ \\
\hline 25 & $1.54 \mathrm{E}-03$ & $7.76 \mathrm{E}-04$ & $1.544 \mathrm{E}-03$ & $3.67 \mathrm{E}-02$ \\
\hline
\end{tabular}


Table 7.9. 200 SCCM

\begin{tabular}{|c|c|c|c|c||}
\hline $\begin{array}{c}\mathbf{d}_{\mathbf{p}} \\
\boldsymbol{\mu m}\end{array}$ & $\begin{array}{c}\mathbf{h}_{\mathbf{s}} \\
\mathbf{c m}\end{array}$ & $\begin{array}{c}\mathbf{V}_{\mathbf{1}}\left(\mathbf{t}_{\mathbf{s}} \mathbf{\mathbf { m } / \mathbf { s }}\right. \\
\mathbf{\text { Max. }}\end{array}$ & $\begin{array}{c}\text { Height } \\
\mathbf{c m}\end{array}$ & $\mathbf{R e}_{\mathbf{p}}$ \\
\hline 14 & $3.07 \mathrm{E}-03$ & $5.30 \mathrm{E}-02$ & $2.719 \mathrm{E}-02$ & $5.38 \mathrm{E}-03$ \\
\hline 15 & $3.07 \mathrm{E}-03$ & $4.90 \mathrm{E}-02$ & $2.175 \mathrm{E}-02$ & $1.88 \mathrm{E}-03$ \\
\hline 16 & $3.07 \mathrm{E}-03$ & $4.54 \mathrm{E}-02$ & $1.848 \mathrm{E}-02$ & $1.71 \mathrm{E}-03$ \\
\hline 17 & $3.07 \mathrm{E}-03$ & $4.21 \mathrm{E}-02$ & $1.600 \mathrm{E}-02$ & $5.38 \mathrm{E}-03$ \\
\hline 18 & $3.07 \mathrm{E}-03$ & $3.91 \mathrm{E}-02$ & $1.401 \mathrm{E}-02$ & $9.15 \mathrm{E}-03$ \\
\hline 19 & $3.07 \mathrm{E}-03$ & $3.64 \mathrm{E}-02$ & $1.236 \mathrm{E}-02$ & $1.30 \mathrm{E}-02$ \\
\hline 20 & $3.07 \mathrm{E}-03$ & $3.39 \mathrm{E}-02$ & $1.098 \mathrm{E}-02$ & $1.69 \mathrm{E}-02$ \\
\hline 21 & $3.07 \mathrm{E}-03$ & $3.15 \mathrm{E}-02$ & $9.818 \mathrm{E}-03$ & $2.10 \mathrm{E}-02$ \\
\hline 22 & $3.07 \mathrm{E}-03$ & $2.93 \mathrm{E}-02$ & $8.824 \mathrm{E}-03$ & $2.51 \mathrm{E}-02$ \\
\hline 23 & $3.07 \mathrm{E}-03$ & $2.73 \mathrm{E}-02$ & $7.969 \mathrm{E}-03$ & $2.93 \mathrm{E}-02$ \\
\hline 24 & $3.07 \mathrm{E}-03$ & $2.53 \mathrm{E}-02$ & $7.231 \mathrm{E}-03$ & $3.37 \mathrm{E}-02$ \\
\hline 25 & $3.07 \mathrm{E}-03$ & $2.34 \mathrm{E}-02$ & $6.589 \mathrm{E}-03$ & $3.81 \mathrm{E}-02$ \\
\hline 26 & $3.07 \mathrm{E}-03$ & $2.16 \mathrm{E}-02$ & $6.028 \mathrm{E}-03$ & $4.27 \mathrm{E}-02$ \\
\hline 27 & $3.07 \mathrm{E}-03$ & $1.98 \mathrm{E}-02$ & $5.535 \mathrm{E}-03$ & $4.75 \mathrm{E}-02$ \\
\hline 28 & $3.07 \mathrm{E}-03$ & $1.80 \mathrm{E}-02$ & $5.101 \mathrm{E}-03$ & $5.24 \mathrm{E}-02$ \\
\hline 29 & $3.07 \mathrm{E}-03$ & $1.63 \mathrm{E}-02$ & $4.716 \mathrm{E}-03$ & $5.75 \mathrm{E}-02$ \\
\hline 30 & $3.07 \mathrm{E}-03$ & $1.46 \mathrm{E}-02$ & $4.373 \mathrm{E}-03$ & $6.28 \mathrm{E}-02$ \\
\hline 31 & $3.07 \mathrm{E}-03$ & $1.28 \mathrm{E}-02$ & $4.067 \mathrm{E}-03$ & $6.85 \mathrm{E}-02$ \\
\hline 32 & $3.07 \mathrm{E}-03$ & $1.09 \mathrm{E}-02$ & $3.794 \mathrm{E}-03$ & $7.45 \mathrm{E}-02$ \\
\hline 33 & $3.07 \mathrm{E}-03$ & $8.91 \mathrm{E}-03$ & $3.549 \mathrm{E}-03$ & $8.11 \mathrm{E}-02$ \\
\hline 34 & $3.07 \mathrm{E}-03$ & $6.57 \mathrm{E}-03$ & $3.330 \mathrm{E}-03$ & $8.87 \mathrm{E}-02$ \\
\hline 35 & $3.07 \mathrm{E}-03$ & $3.35 \mathrm{E}-03$ & $3.137 \mathrm{E}-03$ & $9.86 \mathrm{E}-02$ \\
\hline
\end{tabular}


Table 7.10. 300 SCCM

\begin{tabular}{|c|c|c|c|c|}
\hline $\begin{array}{r}d_{p} \\
\mu m\end{array}$ & $\begin{array}{l}\mathbf{h}_{\mathrm{s}} \\
\mathbf{c m}\end{array}$ & $\begin{array}{c}\mathbf{V}_{1}\left(\mathbf{t}_{\mathbf{s}}\right) \\
\mathbf{m} / \mathbf{s}\end{array}$ & $\begin{array}{c}\text { Max. } \\
\text { Height } \\
\text { cm }\end{array}$ & $\mathbf{R e}_{\mathrm{p}}$ \\
\hline 17 & $4.61 \mathrm{E}-03$ & $6.65 \mathrm{E}-02$ & 5.203E-02 & $4.45 \mathrm{E}-03$ \\
\hline 18 & $4.61 \mathrm{E}-03$ & $6.23 \mathrm{E}-02$ & $4.020 \mathrm{E}-02$ & $9.61 \mathrm{E}-03$ \\
\hline 19 & $4.61 \mathrm{E}-03$ & $5.84 \mathrm{E}-02$ & $3.396 \mathrm{E}-02$ & $1.48 \mathrm{E}-02$ \\
\hline 20 & $4.61 \mathrm{E}-03$ & 5.49E-02 & $2.939 \mathrm{E}-02$ & $2.02 \mathrm{E}-02$ \\
\hline 21 & $4.61 \mathrm{E}-03$ & $5.17 \mathrm{E}-02$ & $2.578 \mathrm{E}-02$ & $2.56 \mathrm{E}-02$ \\
\hline 22 & $4.61 \mathrm{E}-03$ & $4.86 \mathrm{E}-02$ & $2.284 \mathrm{E}-02$ & $3.11 \mathrm{E}-02$ \\
\hline 23 & $4.61 \mathrm{E}-03$ & $4.58 \mathrm{E}-02$ & $2.038 \mathrm{E}-02$ & $3.66 \mathrm{E}-02$ \\
\hline 24 & $4.61 \mathrm{E}-03$ & 4.32E-02 & $1.831 \mathrm{E}-02$ & $4.23 \mathrm{E}-02$ \\
\hline 25 & $4.61 \mathrm{E}-03$ & $4.07 \mathrm{E}-02$ & $1.654 \mathrm{E}-02$ & $4.80 \mathrm{E}-02$ \\
\hline 26 & $4.61 \mathrm{E}-03$ & $3.84 \mathrm{E}-02$ & $1.501 \mathrm{E}-02$ & $5.39 \mathrm{E}-02$ \\
\hline 27 & $4.61 \mathrm{E}-03$ & $3.62 \mathrm{E}-02$ & $1.369 \mathrm{E}-02$ & $5.98 \mathrm{E}-02$ \\
\hline 28 & $4.61 \mathrm{E}-03$ & $3.41 \mathrm{E}-02$ & $1.253 \mathrm{E}-02$ & $6.58 \mathrm{E}-02$ \\
\hline 29 & $4.61 \mathrm{E}-03$ & $3.21 \mathrm{E}-02$ & $1.152 \mathrm{E}-02$ & $7.20 \mathrm{E}-02$ \\
\hline 30 & $4.61 \mathrm{E}-03$ & $3.01 \mathrm{E}-02$ & $1.062 \mathrm{E}-02$ & $7.82 \mathrm{E}-02$ \\
\hline 31 & $4.61 \mathrm{E}-03$ & $2.82 \mathrm{E}-02$ & 9.829E-03 & $8.46 \mathrm{E}-02$ \\
\hline 32 & $4.61 \mathrm{E}-03$ & $2.64 \mathrm{E}-02$ & $9.121 \mathrm{E}-03$ & $9.11 \mathrm{E}-02$ \\
\hline 33 & $4.61 \mathrm{E}-03$ & $2.46 \mathrm{E}-02$ & $8.488 \mathrm{E}-03$ & $9.78 \mathrm{E}-02$ \\
\hline 34 & $4.61 \mathrm{E}-03$ & $2.28 \mathrm{E}-02$ & $7.920 \mathrm{E}-03$ & $1.05 \mathrm{E}-01$ \\
\hline 35 & $4.61 \mathrm{E}-03$ & $2.11 \mathrm{E}-02$ & $7.408 \mathrm{E}-03$ & $1.12 \mathrm{E}-01$ \\
\hline 36 & $4.61 \mathrm{E}-03$ & $1.93 \mathrm{E}-02$ & $6.944 \mathrm{E}-03$ & $1.19 \mathrm{E}-01$ \\
\hline 37 & $4.61 \mathrm{E}-03$ & $1.76 \mathrm{E}-02$ & $6.525 \mathrm{E}-03$ & $1.26 \mathrm{E}-01$ \\
\hline 38 & $4.61 \mathrm{E}-03$ & $1.58 \mathrm{E}-02$ & $6.143 \mathrm{E}-03$ & $1.34 \mathrm{E}-01$ \\
\hline 39 & $4.61 \mathrm{E}-03$ & $1.39 \mathrm{E}-02$ & $5.795 \mathrm{E}-03$ & $1.42 \mathrm{E}-01$ \\
\hline 40 & $4.61 \mathrm{E}-03$ & $1.20 \mathrm{E}-02$ & $5.478 \mathrm{E}-03$ & $1.51 \mathrm{E}-01$ \\
\hline 41 & $4.61 \mathrm{E}-03$ & $9.78 \mathrm{E}-03$ & $5.188 \mathrm{E}-03$ & $1.61 \mathrm{E}-01$ \\
\hline 42 & $4.61 \mathrm{E}-03$ & $7.23 \mathrm{E}-03$ & $4.924 \mathrm{E}-03$ & $1.72 \mathrm{E}-01$ \\
\hline 43 & $4.61 \mathrm{E}-03$ & $3.62 \mathrm{E}-03$ & $4.689 \mathrm{E}-03$ & $1.86 \mathrm{E}-01$ \\
\hline
\end{tabular}


Table 7.11. 400 SCCM

\begin{tabular}{|c|c|c|c|c||}
\hline $\begin{array}{c}\mathbf{d}_{\mathbf{p}} \\
\boldsymbol{\mu m}\end{array}$ & $\begin{array}{c}\mathbf{h}_{\mathbf{s}} \\
\mathbf{c m}\end{array}$ & $\begin{array}{c}\left.\mathbf{V}_{\mathbf{1}} \mathbf{( t} \mathbf{s}\right) \\
\mathbf{m} / \mathbf{s}\end{array}$ & $\begin{array}{c}\text { Max. } \\
\text { Height } \\
\mathbf{c m}\end{array}$ & $\mathbf{R e}_{\mathbf{p}}$ \\
\hline 20 & $6.15 \mathrm{E}-03$ & $7.57 \mathrm{E}-02$ & $7.034 \mathrm{E}-02$ & $2.36 \mathrm{E}-02$ \\
\hline 21 & $6.15 \mathrm{E}-03$ & $7.16 \mathrm{E}-02$ & $5.727 \mathrm{E}-02$ & $3.04 \mathrm{E}-02$ \\
\hline 22 & $6.15 \mathrm{E}-03$ & $6.77 \mathrm{E}-02$ & $4.888 \mathrm{E}-02$ & $3.73 \mathrm{E}-02$ \\
\hline 23 & $6.15 \mathrm{E}-03$ & $6.42 \mathrm{E}-02$ & $4.259 \mathrm{E}-02$ & $4.43 \mathrm{E}-02$ \\
\hline 24 & $6.15 \mathrm{E}-03$ & $6.09 \mathrm{E}-02$ & $3.758 \mathrm{E}-02$ & $5.14 \mathrm{E}-02$ \\
\hline 25 & $6.15 \mathrm{E}-03$ & $5.77 \mathrm{E}-02$ & $3.348 \mathrm{E}-02$ & $5.85 \mathrm{E}-02$ \\
\hline 26 & $6.15 \mathrm{E}-03$ & $5.48 \mathrm{E}-02$ & $3.005 \mathrm{E}-02$ & $6.57 \mathrm{E}-02$ \\
\hline 27 & $6.15 \mathrm{E}-03$ & $5.21 \mathrm{E}-02$ & $2.714 \mathrm{E}-02$ & $7.31 \mathrm{E}-02$ \\
\hline 28 & $6.15 \mathrm{E}-03$ & $4.95 \mathrm{E}-02$ & $2.464 \mathrm{E}-02$ & $8.05 \mathrm{E}-02$ \\
\hline 29 & $6.15 \mathrm{E}-03$ & $4.70 \mathrm{E}-02$ & $2.249 \mathrm{E}-02$ & $8.79 \mathrm{E}-02$ \\
\hline 30 & $6.15 \mathrm{E}-03$ & $4.47 \mathrm{E}-02$ & $2.060 \mathrm{E}-02$ & $9.55 \mathrm{E}-02$ \\
\hline 31 & $6.15 \mathrm{E}-03$ & $4.24 \mathrm{E}-02$ & $1.895 \mathrm{E}-02$ & $1.03 \mathrm{E}-01$ \\
\hline 32 & $6.15 \mathrm{E}-03$ & $4.03 \mathrm{E}-02$ & $1.750 \mathrm{E}-02$ & $1.11 \mathrm{E}-01$ \\
\hline 33 & $6.15 \mathrm{E}-03$ & $3.82 \mathrm{E}-02$ & $1.621 \mathrm{E}-02$ & $1.19 \mathrm{E}-01$ \\
\hline 34 & $6.15 \mathrm{E}-03$ & $3.62 \mathrm{E}-02$ & $1.505 \mathrm{E}-02$ & $1.27 \mathrm{E}-01$ \\
\hline 35 & $6.15 \mathrm{E}-03$ & $3.43 \mathrm{E}-02$ & $1.402 \mathrm{E}-02$ & $1.35 \mathrm{E}-01$ \\
\hline 36 & $6.15 \mathrm{E}-03$ & $3.24 \mathrm{E}-02$ & $1.310 \mathrm{E}-02$ & $1.43 \mathrm{E}-01$ \\
\hline 37 & $6.15 \mathrm{E}-03$ & $3.06 \mathrm{E}-02$ & $1.226 \mathrm{E}-02$ & $1.52 \mathrm{E}-01$ \\
\hline 38 & $6.15 \mathrm{E}-03$ & $2.88 \mathrm{E}-02$ & $1.151 \mathrm{E}-02$ & $1.60 \mathrm{E}-01$ \\
\hline 39 & $6.15 \mathrm{E}-03$ & $2.70 \mathrm{E}-02$ & $1.082 \mathrm{E}-02$ & $1.69 \mathrm{E}-01$ \\
\hline 40 & $6.15 \mathrm{E}-03$ & $2.52 \mathrm{E}-02$ & $1.019 \mathrm{E}-02$ & $1.78 \mathrm{E}-01$ \\
\hline 41 & $6.15 \mathrm{E}-03$ & $2.35 \mathrm{E}-02$ & $9.622 \mathrm{E}-03$ & $1.87 \mathrm{E}-01$ \\
\hline 42 & $6.15 \mathrm{E}-03$ & $2.17 \mathrm{E}-02$ & $9.098 \mathrm{E}-03$ & $1.96 \mathrm{E}-01$ \\
\hline 43 & $6.15 \mathrm{E}-03$ & $1.99 \mathrm{E}-02$ & $8.618 \mathrm{E}-03$ & $2.06 \mathrm{E}-01$ \\
\hline 44 & $6.15 \mathrm{E}-03$ & $1.81 \mathrm{E}-02$ & $8.176 \mathrm{E}-03$ & $2.15 \mathrm{E}-01$ \\
\hline 45 & $6.15 \mathrm{E}-03$ & $1.63 \mathrm{E}-02$ & $7.769 \mathrm{E}-03$ & $2.26 \mathrm{E}-01$ \\
\hline 46 & $6.15 \mathrm{E}-03$ & $1.43 \mathrm{E}-02$ & $7.393 \mathrm{E}-03$ & $2.37 \mathrm{E}-01$ \\
\hline 47 & $6.15 \mathrm{E}-03$ & $1.22 \mathrm{E}-02$ & $7.046 \mathrm{E}-03$ & $2.48 \mathrm{E}-01$ \\
\hline 48 & $6.15 \mathrm{E}-03$ & $9.80 \mathrm{E}-03$ & $6.726 \mathrm{E}-03$ & $2.61 \mathrm{E}-01$ \\
\hline 49 & $6.15 \mathrm{E}-03$ & $6.89 \mathrm{E}-03$ & $6.431 \mathrm{E}-03$ & $2.75 \mathrm{E}-01$ \\
\hline 50 & $6.15 \mathrm{E}-03$ & $2.06 \mathrm{E}-03$ & $6.171 \mathrm{E}-03$ & $2.97 \mathrm{E}-01$ \\
\hline
\end{tabular}


Table 7.12. 500 SCCM

\begin{tabular}{|c|c|c|c|c|}
\hline $\begin{array}{c}d_{p} \\
\mu m\end{array}$ & $\begin{array}{l}\mathbf{h}_{\mathrm{s}} \\
\mathbf{c m}\end{array}$ & $\begin{array}{c}\mathbf{V}_{1}\left(\mathbf{t}_{\mathbf{s}}\right) \\
\mathbf{m} / \mathbf{s}\end{array}$ & $\begin{array}{c}\text { Max. } \\
\text { Height } \\
\text { cm }\end{array}$ & $\operatorname{Re}_{p}$ \\
\hline 22 & $7.68 \mathrm{E}-03$ & $8.68 \mathrm{E}-02$ & $1.081 \mathrm{E}-01$ & $4.37 \mathrm{E}-02$ \\
\hline 23 & $7.68 \mathrm{E}-03$ & $8.24 \mathrm{E}-02$ & $8.418 \mathrm{E}-02$ & $5.21 \mathrm{E}-02$ \\
\hline 24 & $7.68 \mathrm{E}-03$ & $7.84 \mathrm{E}-02$ & $7.105 \mathrm{E}-02$ & $6.06 \mathrm{E}-02$ \\
\hline 25 & $7.68 \mathrm{E}-03$ & $7.46 \mathrm{E}-02$ & $6.159 \mathrm{E}-02$ & $6.92 \mathrm{E}-02$ \\
\hline 26 & $7.68 \mathrm{E}-03$ & $7.11 \mathrm{E}-02$ & $5.421 \mathrm{E}-02$ & 7.79E-02 \\
\hline 27 & $7.68 \mathrm{E}-03$ & $6.78 \mathrm{E}-02$ & $4.824 \mathrm{E}-02$ & $8.66 \mathrm{E}-02$ \\
\hline 28 & $7.68 \mathrm{E}-03$ & $6.47 \mathrm{E}-02$ & 4.329E-02 & $9.54 \mathrm{E}-02$ \\
\hline 29 & $7.68 \mathrm{E}-03$ & $6.18 \mathrm{E}-02$ & $3.911 \mathrm{E}-02$ & $1.04 \mathrm{E}-01$ \\
\hline 30 & $7.68 \mathrm{E}-03$ & $5.90 \mathrm{E}-02$ & $3.553 \mathrm{E}-02$ & $1.13 \mathrm{E}-01$ \\
\hline 31 & $7.68 \mathrm{E}-03$ & 5.64E-02 & $3.245 \mathrm{E}-02$ & $1.22 \mathrm{E}-01$ \\
\hline 32 & $7.68 \mathrm{E}-03$ & $5.38 \mathrm{E}-02$ & $2.976 \mathrm{E}-02$ & $1.32 \mathrm{E}-01$ \\
\hline 33 & $7.68 \mathrm{E}-03$ & 5.14E-02 & $2.741 \mathrm{E}-02$ & $1.41 \mathrm{E}-01$ \\
\hline 34 & $7.68 \mathrm{E}-03$ & 4.91E-02 & $2.533 \mathrm{E}-02$ & $1.50 \mathrm{E}-01$ \\
\hline 35 & $7.68 \mathrm{E}-03$ & 4.69E-02 & $2.349 \mathrm{E}-02$ & $1.60 \mathrm{E}-01$ \\
\hline 36 & $7.68 \mathrm{E}-03$ & $4.48 \mathrm{E}-02$ & $2.185 \mathrm{E}-02$ & $1.69 \mathrm{E}-01$ \\
\hline 37 & $7.68 \mathrm{E}-03$ & $4.27 \mathrm{E}-02$ & $2.038 \mathrm{E}-02$ & $1.79 \mathrm{E}-01$ \\
\hline 38 & $7.68 \mathrm{E}-03$ & $4.07 \mathrm{E}-02$ & $1.905 \mathrm{E}-02$ & $1.88 \mathrm{E}-01$ \\
\hline 39 & $7.68 \mathrm{E}-03$ & $3.87 \mathrm{E}-02$ & $1.786 \mathrm{E}-02$ & $1.98 \mathrm{E}-01$ \\
\hline 40 & $7.68 \mathrm{E}-03$ & $3.68 \mathrm{E}-02$ & $1.677 \mathrm{E}-02$ & $2.08 \mathrm{E}-01$ \\
\hline 41 & $7.68 \mathrm{E}-03$ & $3.50 \mathrm{E}-02$ & $1.579 \mathrm{E}-02$ & $2.18 \mathrm{E}-01$ \\
\hline 42 & $7.68 \mathrm{E}-03$ & $3.32 \mathrm{E}-02$ & $1.489 \mathrm{E}-02$ & $2.29 \mathrm{E}-01$ \\
\hline 43 & $7.68 \mathrm{E}-03$ & $3.14 \mathrm{E}-02$ & $1.407 \mathrm{E}-02$ & $2.39 \mathrm{E}-01$ \\
\hline 44 & $7.68 \mathrm{E}-03$ & $2.96 \mathrm{E}-02$ & $1.332 \mathrm{E}-02$ & $2.50 \mathrm{E}-01$ \\
\hline 45 & $7.68 \mathrm{E}-03$ & $2.78 \mathrm{E}-02$ & $1.262 \mathrm{E}-02$ & $2.60 \mathrm{E}-01$ \\
\hline 46 & $7.68 \mathrm{E}-03$ & $2.61 \mathrm{E}-02$ & 1.199E-02 & $2.71 \mathrm{E}-01$ \\
\hline 47 & $7.68 \mathrm{E}-03$ & $2.43 \mathrm{E}-02$ & 1.140E-02 & $2.83 \mathrm{E}-01$ \\
\hline 48 & $7.68 \mathrm{E}-03$ & $2.25 \mathrm{E}-02$ & $1.085 \mathrm{E}-02$ & $2.94 \mathrm{E}-01$ \\
\hline 49 & $7.68 \mathrm{E}-03$ & $2.07 \mathrm{E}-02$ & $1.035 \mathrm{E}-02$ & $3.06 \mathrm{E}-01$ \\
\hline 50 & $7.68 \mathrm{E}-03$ & $1.89 \mathrm{E}-02$ & $9.877 \mathrm{E}-03$ & $3.18 \mathrm{E}-01$ \\
\hline 51 & $7.68 \mathrm{E}-03$ & $1.69 \mathrm{E}-02$ & $9.440 \mathrm{E}-03$ & $3.31 \mathrm{E}-01$ \\
\hline 52 & $7.68 \mathrm{E}-03$ & 1.49E-02 & $9.034 \mathrm{E}-03$ & 3.44E-01 \\
\hline 53 & $7.68 \mathrm{E}-03$ & $1.27 \mathrm{E}-02$ & $8.656 \mathrm{E}-03$ & $3.59 \mathrm{E}-01$ \\
\hline 54 & $7.68 \mathrm{E}-03$ & $1.01 \mathrm{E}-02$ & $8.304 \mathrm{E}-03$ & $3.74 \mathrm{E}-01$ \\
\hline 55 & $7.68 \mathrm{E}-03$ & $7.02 \mathrm{E}-03$ & 7.979E-03 & $3.92 \mathrm{E}-01$ \\
\hline 56 & $7.68 \mathrm{E}-03$ & $1.31 \mathrm{E}-03$ & $7.692 \mathrm{E}-03$ & $4.20 \mathrm{E}-01$ \\
\hline
\end{tabular}


Table 7.13. 600 SCCM

\begin{tabular}{|c|c|c|c|c|}
\hline $\begin{array}{r}d_{p} \\
\mu \mathbf{m}\end{array}$ & $\begin{array}{l}\mathbf{h}_{\mathrm{s}} \\
\mathbf{c m}\end{array}$ & $\begin{array}{c}\mathbf{V}_{\mathbf{1}}\left(\mathbf{t}_{\mathbf{s}}\right) \\
\mathbf{m} / \mathbf{s}\end{array}$ & $\begin{array}{c}\text { Max. } \\
\text { Height } \\
\text { cm }\end{array}$ & $\mathbf{R e}_{\mathrm{p}}$ \\
\hline 25 & $9.22 \mathrm{E}-03$ & $9.15 \mathrm{E}-02$ & $1.121 \mathrm{E}-01$ & $7.99 \mathrm{E}-02$ \\
\hline 26 & $9.22 \mathrm{E}-03$ & $8.74 \mathrm{E}-02$ & $9.420 \mathrm{E}-02$ & $9.01 \mathrm{E}-02$ \\
\hline 27 & $9.22 \mathrm{E}-03$ & $8.35 \mathrm{E}-02$ & $8.150 \mathrm{E}-02$ & $1.00 \mathrm{E}-01$ \\
\hline 28 & $9.22 \mathrm{E}-03$ & 7.99E-02 & 7.171E-02 & $1.11 \mathrm{E}-01$ \\
\hline 29 & $9.22 \mathrm{E}-03$ & $7.64 \mathrm{E}-02$ & $6.382 \mathrm{E}-02$ & $1.21 \mathrm{E}-01$ \\
\hline 30 & $9.22 \mathrm{E}-03$ & $7.32 \mathrm{E}-02$ & $5.730 \mathrm{E}-02$ & $1.31 \mathrm{E}-01$ \\
\hline 31 & $9.22 \mathrm{E}-03$ & $7.02 \mathrm{E}-02$ & 5.182E-02 & $1.42 \mathrm{E}-01$ \\
\hline 32 & $9.22 \mathrm{E}-03$ & $6.72 \mathrm{E}-02$ & 4.714E-02 & $1.52 \mathrm{E}-01$ \\
\hline 33 & $9.22 \mathrm{E}-03$ & $6.45 \mathrm{E}-02$ & 4.310E-02 & $1.63 \mathrm{E}-01$ \\
\hline 34 & $9.22 \mathrm{E}-03$ & $6.18 \mathrm{E}-02$ & 3.959E-02 & $1.74 \mathrm{E}-01$ \\
\hline 35 & $9.22 \mathrm{E}-03$ & 5.93E-02 & $3.651 \mathrm{E}-02$ & $1.85 \mathrm{E}-01$ \\
\hline 36 & $9.22 \mathrm{E}-03$ & 5.69E-02 & 3.379E-02 & $1.96 \mathrm{E}-01$ \\
\hline 37 & $9.22 \mathrm{E}-03$ & $5.45 \mathrm{E}-02$ & $3.137 \mathrm{E}-02$ & $2.07 \mathrm{E}-01$ \\
\hline 38 & $9.22 \mathrm{E}-03$ & $5.23 \mathrm{E}-02$ & $2.922 \mathrm{E}-02$ & $2.18 \mathrm{E}-01$ \\
\hline 39 & $9.22 \mathrm{E}-03$ & $5.01 \mathrm{E}-02$ & 2.729E-02 & $2.29 \mathrm{E}-01$ \\
\hline 40 & $9.22 \mathrm{E}-03$ & $4.80 \mathrm{E}-02$ & $2.555 \mathrm{E}-02$ & $2.40 \mathrm{E}-01$ \\
\hline 41 & $9.22 \mathrm{E}-03$ & $4.60 \mathrm{E}-02$ & $2.397 \mathrm{E}-02$ & $2.52 \mathrm{E}-01$ \\
\hline 42 & $9.22 \mathrm{E}-03$ & $4.40 \mathrm{E}-02$ & $2.255 \mathrm{E}-02$ & $2.63 \mathrm{E}-01$ \\
\hline 43 & $9.22 \mathrm{E}-03$ & $4.20 \mathrm{E}-02$ & $2.125 \mathrm{E}-02$ & $2.75 \mathrm{E}-01$ \\
\hline 44 & $9.22 \mathrm{E}-03$ & $4.01 \mathrm{E}-02$ & $2.006 \mathrm{E}-02$ & $2.86 \mathrm{E}-01$ \\
\hline 45 & $9.22 \mathrm{E}-03$ & $3.83 \mathrm{E}-02$ & $1.898 \mathrm{E}-02$ & $2.98 \mathrm{E}-01$ \\
\hline 46 & $9.22 \mathrm{E}-03$ & 3.64E-02 & $1.798 \mathrm{E}-02$ & $3.10 \mathrm{E}-01$ \\
\hline 47 & $9.22 \mathrm{E}-03$ & $3.46 \mathrm{E}-02$ & $1.706 \mathrm{E}-02$ & $3.23 \mathrm{E}-01$ \\
\hline 48 & $9.22 \mathrm{E}-03$ & 3.29E-02 & $1.621 \mathrm{E}-02$ & $3.35 \mathrm{E}-01$ \\
\hline 49 & $9.22 \mathrm{E}-03$ & $3.11 \mathrm{E}-02$ & $1.543 \mathrm{E}-02$ & $3.48 \mathrm{E}-01$ \\
\hline 50 & $9.22 \mathrm{E}-03$ & $2.93 \mathrm{E}-02$ & $1.470 \mathrm{E}-02$ & $3.60 \mathrm{E}-01$ \\
\hline 51 & $9.22 \mathrm{E}-03$ & $2.76 \mathrm{E}-02$ & $1.403 \mathrm{E}-02$ & $3.73 \mathrm{E}-01$ \\
\hline 52 & $9.22 \mathrm{E}-03$ & $2.58 \mathrm{E}-02$ & $1.340 \mathrm{E}-02$ & $3.87 \mathrm{E}-01$ \\
\hline 53 & $9.22 \mathrm{E}-03$ & $2.40 \mathrm{E}-02$ & $1.282 \mathrm{E}-02$ & $4.00 \mathrm{E}-01$ \\
\hline 54 & $9.22 \mathrm{E}-03$ & $2.22 \mathrm{E}-02$ & $1.227 \mathrm{E}-02$ & $4.14 \mathrm{E}-01$ \\
\hline 55 & $9.22 \mathrm{E}-03$ & $2.03 \mathrm{E}-02$ & $1.176 \mathrm{E}-02$ & $4.28 \mathrm{E}-01$ \\
\hline 56 & $9.22 \mathrm{E}-03$ & $1.84 \mathrm{E}-02$ & $1.129 \mathrm{E}-02$ & $4.43 \mathrm{E}-01$ \\
\hline 57 & $9.22 \mathrm{E}-03$ & $1.63 \mathrm{E}-02$ & $1.084 \mathrm{E}-02$ & $4.59 \mathrm{E}-01$ \\
\hline 58 & $9.22 \mathrm{E}-03$ & $1.41 \mathrm{E}-02$ & $1.042 \mathrm{E}-02$ & $4.75 \mathrm{E}-01$ \\
\hline 59 & $9.22 \mathrm{E}-03$ & $1.16 \mathrm{E}-02$ & $1.003 \mathrm{E}-02$ & $4.93 \mathrm{E}-01$ \\
\hline 60 & $9.22 \mathrm{E}-03$ & $8.63 \mathrm{E}-03$ & $9.667 \mathrm{E}-03$ & $5.13 \mathrm{E}-01$ \\
\hline 61 & $9.22 \mathrm{E}-03$ & $4.35 \mathrm{E}-03$ & $9.332 \mathrm{E}-03$ & $5.38 \mathrm{E}-01$ \\
\hline
\end{tabular}


Table 7.14. 700 SCCM

\begin{tabular}{|c|c|c|c|c|}
\hline $\begin{array}{l}d_{p} \\
\mu m\end{array}$ & $\begin{array}{l}\mathbf{h}_{\mathrm{s}} \\
\mathbf{c m}\end{array}$ & $\begin{array}{c}\mathrm{V}_{1}\left(\mathbf{t}_{\mathrm{s}}\right) \\
\mathrm{m} / \mathrm{s}\end{array}$ & $\begin{array}{c}\text { Max. Height } \\
\text { cm }\end{array}$ & $\operatorname{Re}_{p}$ \\
\hline 26 & $1.08 \mathrm{E}-02$ & $1.04 \mathrm{E}-01$ & $1.798 \mathrm{E}-01$ & $1.02 \mathrm{E}-01$ \\
\hline 27 & $1.08 \mathrm{E}-02$ & $9.91 \mathrm{E}-02$ & $1.387 \mathrm{E}-01$ & $1.14 \mathrm{E}-01$ \\
\hline 28 & $1.08 \mathrm{E}-02$ & $9.50 \mathrm{E}-02$ & $1.168 \mathrm{E}-01$ & $1.26 \mathrm{E}-01$ \\
\hline 29 & $1.08 \mathrm{E}-02$ & $9.11 \mathrm{E}-02$ & $1.012 \mathrm{E}-01$ & $1.37 \mathrm{E}-01$ \\
\hline 30 & $1.08 \mathrm{E}-02$ & $8.74 \mathrm{E}-02$ & 8.917E-02 & $1.49 \mathrm{E}-01$ \\
\hline 31 & $1.08 \mathrm{E}-02$ & $8.39 \mathrm{E}-02$ & 7.949E-02 & $1.61 \mathrm{E}-01$ \\
\hline 32 & $1.08 \mathrm{E}-02$ & $8.06 \mathrm{E}-02$ & 7.149E-02 & $1.73 \mathrm{E}-01$ \\
\hline 33 & $1.08 \mathrm{E}-02$ & $7.74 \mathrm{E}-02$ & $6.475 \mathrm{E}-02$ & $1.85 \mathrm{E}-01$ \\
\hline 34 & $1.08 \mathrm{E}-02$ & $7.45 \mathrm{E}-02$ & $5.900 \mathrm{E}-02$ & $1.98 \mathrm{E}-01$ \\
\hline 35 & $1.08 \mathrm{E}-02$ & $7.16 \mathrm{E}-02$ & 5.404E-02 & $2.10 \mathrm{E}-01$ \\
\hline 36 & $1.08 \mathrm{E}-02$ & $6.89 \mathrm{E}-02$ & 4.972E-02 & $2.22 \mathrm{E}-01$ \\
\hline 37 & $1.08 \mathrm{E}-02$ & $6.62 \mathrm{E}-02$ & $4.593 \mathrm{E}-02$ & $2.35 \mathrm{E}-01$ \\
\hline 38 & $1.08 \mathrm{E}-02$ & $6.37 \mathrm{E}-02$ & 4.257E-02 & $2.47 \mathrm{E}-01$ \\
\hline 39 & $1.08 \mathrm{E}-02$ & $6.13 \mathrm{E}-02$ & $3.959 \mathrm{E}-02$ & $2.60 \mathrm{E}-01$ \\
\hline 40 & $1.08 \mathrm{E}-02$ & $5.90 \mathrm{E}-02$ & $3.693 \mathrm{E}-02$ & $2.72 \mathrm{E}-01$ \\
\hline 41 & $1.08 \mathrm{E}-02$ & $5.67 \mathrm{E}-02$ & $3.453 \mathrm{E}-02$ & $2.85 \mathrm{E}-01$ \\
\hline 42 & $1.08 \mathrm{E}-02$ & $5.45 \mathrm{E}-02$ & $3.238 \mathrm{E}-02$ & $2.98 \mathrm{E}-01$ \\
\hline 43 & $1.08 \mathrm{E}-02$ & $5.24 \mathrm{E}-02$ & $3.043 \mathrm{E}-02$ & $3.11 \mathrm{E}-01$ \\
\hline 44 & $1.08 \mathrm{E}-02$ & $5.03 \mathrm{E}-02$ & $2.865 \mathrm{E}-02$ & $3.24 \mathrm{E}-01$ \\
\hline 45 & $1.08 \mathrm{E}-02$ & $4.83 \mathrm{E}-02$ & $2.703 \mathrm{E}-02$ & $3.37 \mathrm{E}-01$ \\
\hline 46 & $1.08 \mathrm{E}-02$ & $4.64 \mathrm{E}-02$ & $2.556 \mathrm{E}-02$ & $3.51 \mathrm{E}-01$ \\
\hline 47 & $1.08 \mathrm{E}-02$ & 4.44E-02 & $2.420 \mathrm{E}-02$ & $3.64 \mathrm{E}-01$ \\
\hline 48 & $1.08 \mathrm{E}-02$ & $4.26 \mathrm{E}-02$ & $2.295 \mathrm{E}-02$ & $3.78 \mathrm{E}-01$ \\
\hline 49 & $1.08 \mathrm{E}-02$ & $4.07 \mathrm{E}-02$ & $2.180 \mathrm{E}-02$ & $3.91 \mathrm{E}-01$ \\
\hline 50 & $1.08 \mathrm{E}-02$ & $3.89 \mathrm{E}-02$ & $2.074 \mathrm{E}-02$ & $4.05 \mathrm{E}-01$ \\
\hline 51 & $1.08 \mathrm{E}-02$ & $3.71 \mathrm{E}-02$ & $1.976 \mathrm{E}-02$ & $4.19 \mathrm{E}-01$ \\
\hline 52 & $1.08 \mathrm{E}-02$ & $3.53 \mathrm{E}-02$ & $1.885 \mathrm{E}-02$ & $4.34 \mathrm{E}-01$ \\
\hline 53 & $1.08 \mathrm{E}-02$ & $3.35 \mathrm{E}-02$ & $1.800 \mathrm{E}-02$ & $4.48 \mathrm{E}-01$ \\
\hline 54 & $1.08 \mathrm{E}-02$ & $3.18 \mathrm{E}-02$ & $1.721 \mathrm{E}-02$ & $4.63 \mathrm{E}-01$ \\
\hline 55 & $1.08 \mathrm{E}-02$ & $3.00 \mathrm{E}-02$ & $1.647 \mathrm{E}-02$ & 4.77E-01 \\
\hline 56 & $1.08 \mathrm{E}-02$ & $2.82 \mathrm{E}-02$ & $1.578 \mathrm{E}-02$ & 4.92E-01 \\
\hline 57 & $1.08 \mathrm{E}-02$ & $2.65 \mathrm{E}-02$ & $1.514 \mathrm{E}-02$ & $5.08 \mathrm{E}-01$ \\
\hline 58 & $1.08 \mathrm{E}-02$ & $2.46 \mathrm{E}-02$ & $1.453 \mathrm{E}-02$ & $5.24 \mathrm{E}-01$ \\
\hline 59 & $1.08 \mathrm{E}-02$ & $2.28 \mathrm{E}-02$ & $1.397 \mathrm{E}-02$ & $5.40 \mathrm{E}-01$ \\
\hline 60 & $1.08 \mathrm{E}-02$ & $2.09 \mathrm{E}-02$ & $1.344 \mathrm{E}-02$ & $5.56 \mathrm{E}-01$ \\
\hline 61 & $1.08 \mathrm{E}-02$ & $1.89 \mathrm{E}-02$ & $1.293 \mathrm{E}-02$ & $5.73 \mathrm{E}-01$ \\
\hline 62 & $1.08 \mathrm{E}-02$ & $1.67 \mathrm{E}-02$ & $1.246 \mathrm{E}-02$ & $5.91 \mathrm{E}-01$ \\
\hline 63 & $1.08 \mathrm{E}-02$ & $1.44 \mathrm{E}-02$ & $1.202 \mathrm{E}-02$ & $6.10 \mathrm{E}-01$ \\
\hline 64 & $1.08 \mathrm{E}-02$ & $1.18 \mathrm{E}-02$ & $1.160 \mathrm{E}-02$ & $6.31 \mathrm{E}-01$ \\
\hline 65 & $1.08 \mathrm{E}-02$ & $8.70 \mathrm{E}-03$ & $1.121 \mathrm{E}-02$ & $6.54 \mathrm{E}-01$ \\
\hline 66 & $1.08 \mathrm{E}-02$ & $4.03 \mathrm{E}-03$ & $1.085 \mathrm{E}-02$ & $6.84 \mathrm{E}-01$ \\
\hline
\end{tabular}


Table 7.15. 800 SCCM

\begin{tabular}{|c|c|c|c|c|}
\hline $\begin{array}{c}d_{p} \\
\mu \mathbf{m}\end{array}$ & $\begin{array}{l}\mathbf{h}_{\mathbf{s}} \\
\mathbf{c m}\end{array}$ & $\begin{array}{c}\mathbf{V}_{1}\left(\mathbf{t}_{\mathbf{s}}\right) \\
\mathbf{m} / \mathbf{s}\end{array}$ & $\begin{array}{c}\text { Max. Height } \\
\text { cm }\end{array}$ & $\mathbf{R e}_{\mathrm{p}}$ \\
\hline 28 & $1.23 \mathrm{E}-02$ & $1.10 \mathrm{E}-01$ & $2.035 \mathrm{E}-01$ & $1.41 \mathrm{E}-01$ \\
\hline 29 & & & & \\
\hline 30 & $23 \mathrm{E}-02$ & 02E-01 & $377 \mathrm{E}-01$ & \\
\hline 31 & & & & \\
\hline 32 & & & & \\
\hline 33 & $23 \mathrm{E}-02$ & 04E-02 & $9.468 \mathrm{E}-02$ & \\
\hline 34 & $23 \mathrm{E}-02$ & $70 \mathrm{E}-02$ & & \\
\hline 35 & $23 \mathrm{E}-02$ & & & \\
\hline 36 & $23 \mathrm{E}-02$ & $8 \mathrm{E}-02$ & & \\
\hline 37 & $3 \mathrm{E}-02$ & 79E-02 & & \\
\hline 38 & & & & \\
\hline 39 & $3 \mathrm{E}-02$ & & & \\
\hline 40 & & & & \\
\hline 41 & & & & \\
\hline 42 & $3 \mathrm{E}-02$ & & & \\
\hline 43 & $23 \mathrm{E}-02$ & & & \\
\hline 44 & & & & \\
\hline 45 & & & & \\
\hline 46 & $23 \mathrm{E}-02$ & & & \\
\hline 47 & & & & \\
\hline 48 & $23 \mathrm{E}-02$ & & & \\
\hline 49 & & & & \\
\hline 50 & & & & \\
\hline 51 & & & & \\
\hline 52 & & & & \\
\hline 53 & & & & \\
\hline 54 & & & & \\
\hline 55 & & & & \\
\hline 56 & & & & \\
\hline 57 & & & & \\
\hline 58 & & & & \\
\hline 59 & $3 \mathrm{E}-02$ & & & \\
\hline 60 & $1.23 \mathrm{E}-02$ & $\mathrm{E}-02$ & & \\
\hline 61 & $1.23 \mathrm{E}-02$ & $2.82 \mathrm{E}-02$ & & \\
\hline 62 & & & & \\
\hline 63 & $1.23 \mathrm{E}-02$ & $2.46 \mathrm{E}-02$ & & \\
\hline 64 & $1.23 \mathrm{E}-02$ & & & \\
\hline 65 & $1.23 \mathrm{E}-02$ & $2.06 \mathrm{E}-02$ & & $7.02 \mathrm{E}-01$ \\
\hline 66 & $23 \mathrm{E}-02$ & & & \\
\hline 67 & $1.23 \mathrm{E}-02$ & & & 7.42E-01 \\
\hline 68 & $1.23 \mathrm{E}-02$ & & & 7.64E-01 \\
\hline 69 & & & & \\
\hline 70 & & & $1.261 \mathrm{E}-02$ & $8.16 \mathrm{E}-01$ \\
\hline
\end{tabular}


Table 7.16. 900 SCCM

\begin{tabular}{|c|c|c|c|c|}
\hline $\begin{array}{l}d_{p} \\
\mu m\end{array}$ & $\begin{array}{l}\mathbf{h}_{\mathrm{s}} \\
\mathbf{c m}\end{array}$ & $\begin{array}{c}\mathbf{V}_{1}\left(\mathbf{t}_{\mathbf{s}}\right) \\
\mathbf{m} / \mathbf{s}\end{array}$ & $\begin{array}{c}\text { Max. Height } \\
\text { cm }\end{array}$ & $\mathbf{R e}_{\mathbf{p}}$ \\
\hline 30 & $1.38 \mathrm{E}-02$ & $1.16 \mathrm{E}-01$ & $2.227 \mathrm{E}-01$ & $1.86 \mathrm{E}-01$ \\
\hline 31 & $1.38 \mathrm{E}-02$ & $1.11 \mathrm{E}-01$ & $1.826 \mathrm{E}-01$ & $2.01 \mathrm{E}-01$ \\
\hline 32 & $1.38 \mathrm{E}-02$ & $1.07 \mathrm{E}-01$ & $1.564 \mathrm{E}-01$ & $2.16 \mathrm{E}-01$ \\
\hline 33 & $1.38 \mathrm{E}-02$ & $1.03 \mathrm{E}-01$ & $1.369 \mathrm{E}-01$ & $2.31 \mathrm{E}-01$ \\
\hline 34 & $1.38 \mathrm{E}-02$ & $9.96 \mathrm{E}-02$ & $1.215 \mathrm{E}-01$ & $2.46 \mathrm{E}-01$ \\
\hline 35 & $1.38 \mathrm{E}-02$ & $9.60 \mathrm{E}-02$ & $1.090 \mathrm{E}-01$ & $2.61 \mathrm{E}-01$ \\
\hline 36 & $1.38 \mathrm{E}-02$ & $9.27 \mathrm{E}-02$ & $9.858 \mathrm{E}-02$ & $2.76 \mathrm{E}-01$ \\
\hline 37 & $1.38 \mathrm{E}-02$ & $8.95 \mathrm{E}-02$ & $8.974 \mathrm{E}-02$ & $2.91 \mathrm{E}-01$ \\
\hline 38 & $1.38 \mathrm{E}-02$ & 8.64E-02 & $8.216 \mathrm{E}-02$ & $3.07 \mathrm{E}-01$ \\
\hline 39 & $1.38 \mathrm{E}-02$ & $8.34 \mathrm{E}-02$ & $7.558 \mathrm{E}-02$ & $3.22 \mathrm{E}-01$ \\
\hline 40 & $1.38 \mathrm{E}-02$ & $8.06 \mathrm{E}-02$ & $6.982 \mathrm{E}-02$ & $3.38 \mathrm{E}-01$ \\
\hline 41 & $1.38 \mathrm{E}-02$ & 7.79E-02 & $6.475 \mathrm{E}-02$ & $3.54 \mathrm{E}-01$ \\
\hline 42 & $1.38 \mathrm{E}-02$ & $7.53 \mathrm{E}-02$ & $6.024 \mathrm{E}-02$ & $3.69 \mathrm{E}-01$ \\
\hline 43 & $1.38 \mathrm{E}-02$ & $7.27 \mathrm{E}-02$ & $5.622 \mathrm{E}-02$ & $3.85 \mathrm{E}-01$ \\
\hline 44 & $1.38 \mathrm{E}-02$ & $7.03 \mathrm{E}-02$ & $5.261 \mathrm{E}-02$ & $4.01 \mathrm{E}-01$ \\
\hline 45 & $1.38 \mathrm{E}-02$ & $6.79 \mathrm{E}-02$ & $4.936 \mathrm{E}-02$ & $4.17 \mathrm{E}-01$ \\
\hline 46 & $1.38 \mathrm{E}-02$ & $6.56 \mathrm{E}-02$ & $4.642 \mathrm{E}-02$ & $4.33 \mathrm{E}-01$ \\
\hline 47 & $1.38 \mathrm{E}-02$ & $6.34 \mathrm{E}-02$ & $4.375 \mathrm{E}-02$ & 4.49E-01 \\
\hline 48 & $1.38 \mathrm{E}-02$ & $6.12 \mathrm{E}-02$ & $4.131 \mathrm{E}-02$ & $4.66 \mathrm{E}-01$ \\
\hline 49 & $1.38 \mathrm{E}-02$ & 5.91E-02 & $3.908 \mathrm{E}-02$ & $4.82 \mathrm{E}-01$ \\
\hline 50 & $1.38 \mathrm{E}-02$ & $5.70 \mathrm{E}-02$ & $3.704 \mathrm{E}-02$ & $4.98 \mathrm{E}-01$ \\
\hline 51 & $1.38 \mathrm{E}-02$ & $5.50 \mathrm{E}-02$ & $3.516 \mathrm{E}-02$ & $5.15 \mathrm{E}-01$ \\
\hline 52 & $1.38 \mathrm{E}-02$ & 5.31E-02 & $3.343 \mathrm{E}-02$ & $5.32 \mathrm{E}-01$ \\
\hline 53 & $1.38 \mathrm{E}-02$ & $5.11 \mathrm{E}-02$ & $3.182 \mathrm{E}-02$ & $5.49 \mathrm{E}-01$ \\
\hline 54 & $1.38 \mathrm{E}-02$ & 4.92E-02 & $3.034 \mathrm{E}-02$ & $5.66 \mathrm{E}-01$ \\
\hline 55 & $1.38 \mathrm{E}-02$ & 4.74E-02 & $2.896 \mathrm{E}-02$ & $5.83 \mathrm{E}-01$ \\
\hline 56 & $1.38 \mathrm{E}-02$ & $4.55 \mathrm{E}-02$ & $2.768 \mathrm{E}-02$ & $6.00 \mathrm{E}-01$ \\
\hline 57 & $1.38 \mathrm{E}-02$ & 4.37E-02 & $2.648 \mathrm{E}-02$ & $6.17 \mathrm{E}-01$ \\
\hline 58 & $1.38 \mathrm{E}-02$ & 4.19E-02 & $2.537 \mathrm{E}-02$ & $6.35 \mathrm{E}-01$ \\
\hline 59 & $1.38 \mathrm{E}-02$ & $4.01 \mathrm{E}-02$ & $2.432 \mathrm{E}-02$ & $6.53 \mathrm{E}-01$ \\
\hline 60 & $1.38 \mathrm{E}-02$ & $3.83 \mathrm{E}-02$ & $2.334 \mathrm{E}-02$ & $6.71 \mathrm{E}-01$ \\
\hline 61 & $1.38 \mathrm{E}-02$ & $3.66 \mathrm{E}-02$ & $2.242 \mathrm{E}-02$ & $6.89 \mathrm{E}-01$ \\
\hline 62 & $1.38 \mathrm{E}-02$ & $3.48 \mathrm{E}-02$ & $2.156 \mathrm{E}-02$ & 7.07E-01 \\
\hline 63 & $1.38 \mathrm{E}-02$ & $3.30 \mathrm{E}-02$ & $2.075 \mathrm{E}-02$ & $7.26 \mathrm{E}-01$ \\
\hline 64 & $1.38 \mathrm{E}-02$ & $3.13 \mathrm{E}-02$ & $1.999 \mathrm{E}-02$ & $7.45 \mathrm{E}-01$ \\
\hline 65 & $1.38 \mathrm{E}-02$ & $2.95 \mathrm{E}-02$ & $1.927 \mathrm{E}-02$ & 7.64E-01 \\
\hline 66 & $1.38 \mathrm{E}-02$ & $2.76 \mathrm{E}-02$ & $1.858 \mathrm{E}-02$ & $7.83 \mathrm{E}-01$ \\
\hline 67 & $1.38 \mathrm{E}-02$ & $2.58 \mathrm{E}-02$ & $1.794 \mathrm{E}-02$ & $8.03 \mathrm{E}-01$ \\
\hline 68 & $1.38 \mathrm{E}-02$ & $2.38 \mathrm{E}-02$ & $1.733 \mathrm{E}-02$ & $8.24 \mathrm{E}-01$ \\
\hline 69 & $1.38 \mathrm{E}-02$ & $2.18 \mathrm{E}-02$ & $1.676 \mathrm{E}-02$ & $8.45 \mathrm{E}-01$ \\
\hline
\end{tabular}


Table 7.16. 900 SCCM

\begin{tabular}{||c|c|c|c|c||}
\hline \hline $\begin{array}{c}\mathbf{d}_{\mathbf{p}} \\
\boldsymbol{\mu m}\end{array}$ & $\begin{array}{c}\mathbf{h}_{\mathbf{s}} \\
\mathbf{c m}\end{array}$ & $\begin{array}{c}\mathbf{V}_{\mathbf{1}}\left(\mathbf{t}_{\mathbf{s}}\right) \\
\mathbf{m} / \mathbf{s}\end{array}$ & $\begin{array}{c}\text { Max. Height } \\
\mathbf{c m}\end{array}$ & $\mathbf{R e}_{\mathbf{p}}$ \\
\hline 70 & $1.38 \mathrm{E}-02$ & $1.97 \mathrm{E}-02$ & $1.621 \mathrm{E}-02$ & $8.66 \mathrm{E}-01$ \\
\hline 71 & $1.38 \mathrm{E}-02$ & $1.75 \mathrm{E}-02$ & $1.569 \mathrm{E}-02$ & $8.89 \mathrm{E}-01$ \\
\hline 72 & $1.38 \mathrm{E}-02$ & $1.50 \mathrm{E}-02$ & $1.520 \mathrm{E}-02$ & $9.13 \mathrm{E}-01$ \\
\hline 73 & $1.38 \mathrm{E}-02$ & $1.23 \mathrm{E}-02$ & $1.474 \mathrm{E}-02$ & $9.39 \mathrm{E}-01$ \\
\hline 74 & $1.38 \mathrm{E}-02$ & $8.84 \mathrm{E}-03$ & $1.430 \mathrm{E}-02$ & $9.68 \mathrm{E}-01$ \\
\hline 75 & $1.38 \mathrm{E}-02$ & $3.38 \mathrm{E}-03$ & $1.390 \mathrm{E}-02$ & $1.01 \mathrm{E}+00$ \\
\hline
\end{tabular}

Table 7.17. 1000 SCCM

\begin{tabular}{|c|c|c|c|c||}
\hline $\begin{array}{c}\mathbf{d}_{\mathbf{p}} \\
\boldsymbol{\mu m}\end{array}$ & $\begin{array}{c}\mathbf{h}_{\mathbf{s}} \\
\mathbf{c m}\end{array}$ & $\begin{array}{c}\mathbf{V}_{\mathbf{1}}\left(\mathbf{t}_{\mathbf{s}} \mathbf{)}\right. \\
\mathbf{m} / \mathbf{s}\end{array}$ & $\begin{array}{c}\text { Max. Height } \\
\mathbf{c m}\end{array}$ & Re $_{\mathbf{p}}$ \\
\hline 31 & $1.54 \mathrm{E}-02$ & $1.25 \mathrm{E}-01$ & $3.411 \mathrm{E}-01$ & $2.20 \mathrm{E}-01$ \\
\hline 32 & $1.54 \mathrm{E}-02$ & $1.20 \mathrm{E}-01$ & $2.380 \mathrm{E}-01$ & $2.37 \mathrm{E}-01$ \\
\hline 33 & $1.54 \mathrm{E}-02$ & $1.16 \mathrm{E}-01$ & $1.996 \mathrm{E}-01$ & $2.53 \mathrm{E}-01$ \\
\hline 34 & $1.54 \mathrm{E}-02$ & $1.12 \mathrm{E}-01$ & $1.726 \mathrm{E}-01$ & $2.70 \mathrm{E}-01$ \\
\hline 35 & $1.54 \mathrm{E}-02$ & $1.08 \mathrm{E}-01$ & $1.521 \mathrm{E}-01$ & $2.86 \mathrm{E}-01$ \\
\hline 36 & $1.54 \mathrm{E}-02$ & $1.05 \mathrm{E}-01$ & $1.357 \mathrm{E}-01$ & $3.03 \mathrm{E}-01$ \\
\hline 37 & $1.54 \mathrm{E}-02$ & $1.01 \mathrm{E}-01$ & $1.222 \mathrm{E}-01$ & $3.20 \mathrm{E}-01$ \\
\hline 38 & $1.54 \mathrm{E}-02$ & $9.77 \mathrm{E}-02$ & $1.109 \mathrm{E}-01$ & $3.37 \mathrm{E}-01$ \\
\hline 39 & $1.54 \mathrm{E}-02$ & $9.45 \mathrm{E}-02$ & $1.013 \mathrm{E}-01$ & $3.54 \mathrm{E}-01$ \\
\hline 40 & $1.54 \mathrm{E}-02$ & $9.14 \mathrm{E}-02$ & $9.300 \mathrm{E}-02$ & $3.71 \mathrm{E}-01$ \\
\hline 41 & $1.54 \mathrm{E}-02$ & $8.84 \mathrm{E}-02$ & $8.577 \mathrm{E}-02$ & $3.88 \mathrm{E}-01$ \\
\hline 42 & $1.54 \mathrm{E}-02$ & $8.56 \mathrm{E}-02$ & $7.942 \mathrm{E}-02$ & $4.05 \mathrm{E}-01$ \\
\hline 43 & $1.54 \mathrm{E}-02$ & $8.28 \mathrm{E}-02$ & $7.381 \mathrm{E}-02$ & $4.22 \mathrm{E}-01$ \\
\hline 44 & $1.54 \mathrm{E}-02$ & $8.02 \mathrm{E}-02$ & $6.881 \mathrm{E}-02$ & $4.40 \mathrm{E}-01$ \\
\hline 45 & $1.54 \mathrm{E}-02$ & $7.76 \mathrm{E}-02$ & $6.434 \mathrm{E}-02$ & $4.57 \mathrm{E}-01$ \\
\hline 46 & $1.54 \mathrm{E}-02$ & $7.51 \mathrm{E}-02$ & $6.032 \mathrm{E}-02$ & $4.75 \mathrm{E}-01$ \\
\hline 47 & $1.54 \mathrm{E}-02$ & $7.27 \mathrm{E}-02$ & $5.669 \mathrm{E}-02$ & $4.92 \mathrm{E}-01$ \\
\hline 48 & $1.54 \mathrm{E}-02$ & $7.04 \mathrm{E}-02$ & $5.340 \mathrm{E}-02$ & $5.10 \mathrm{E}-01$ \\
\hline 49 & $1.54 \mathrm{E}-02$ & $6.81 \mathrm{E}-02$ & $5.040 \mathrm{E}-02$ & $5.28 \mathrm{E}-01$ \\
\hline 50 & $1.54 \mathrm{E}-02$ & $6.59 \mathrm{E}-02$ & $4.766 \mathrm{E}-02$ & $5.46 \mathrm{E}-01$ \\
\hline 51 & $1.54 \mathrm{E}-02$ & $6.38 \mathrm{E}-02$ & $4.515 \mathrm{E}-02$ & $5.64 \mathrm{E}-01$ \\
\hline 52 & $1.54 \mathrm{E}-02$ & $6.17 \mathrm{E}-02$ & $4.285 \mathrm{E}-02$ & $5.82 \mathrm{E}-01$ \\
\hline 53 & $1.54 \mathrm{E}-02$ & $5.96 \mathrm{E}-02$ & $4.072 \mathrm{E}-02$ & $6.00 \mathrm{E}-01$ \\
\hline 54 & $1.54 \mathrm{E}-02$ & $5.76 \mathrm{E}-02$ & $3.876 \mathrm{E}-02$ & $6.18 \mathrm{E}-01$ \\
\hline 55 & $1.54 \mathrm{E}-02$ & $5.56 \mathrm{E}-02$ & $3.695 \mathrm{E}-02$ & $6.37 \mathrm{E}-01$ \\
\hline 56 & $1.54 \mathrm{E}-02$ & $5.37 \mathrm{E}-02$ & $3.526 \mathrm{E}-02$ & $6.55 \mathrm{E}-01$ \\
\hline 57 & $1.54 \mathrm{E}-02$ & $5.18 \mathrm{E}-02$ & $3.369 \mathrm{E}-02$ & $6.74 \mathrm{E}-01$ \\
\hline 58 & $1.54 \mathrm{E}-02$ & $4.99 \mathrm{E}-02$ & $3.223 \mathrm{E}-02$ & $6.93 \mathrm{E}-01$ \\
\hline & & & & \\
\hline
\end{tabular}


Table 7.17. 1000 SCCM

\begin{tabular}{||c|c|c|c|c||}
\hline $\begin{array}{c}\mathbf{d}_{\mathbf{p}} \\
\boldsymbol{\mu m}\end{array}$ & $\begin{array}{c}\mathbf{h}_{\mathbf{s}} \\
\mathbf{c m}\end{array}$ & $\begin{array}{c}\mathbf{V}_{\mathbf{1}}\left(\mathbf{t}_{\mathbf{s}}\right) \\
\mathbf{m} / \mathbf{s}\end{array}$ & $\begin{array}{c}\text { Max. Height } \\
\mathbf{c m}\end{array}$ & $\mathbf{R e}_{\mathbf{p}}$ \\
\hline 59 & $1.54 \mathrm{E}-02$ & $4.81 \mathrm{E}-02$ & $3.087 \mathrm{E}-02$ & $7.12 \mathrm{E}-01$ \\
\hline 60 & $1.54 \mathrm{E}-02$ & $4.63 \mathrm{E}-02$ & $2.960 \mathrm{E}-02$ & $7.31 \mathrm{E}-01$ \\
\hline 61 & $1.54 \mathrm{E}-02$ & $4.45 \mathrm{E}-02$ & $2.840 \mathrm{E}-02$ & $7.50 \mathrm{E}-01$ \\
\hline 62 & $1.54 \mathrm{E}-02$ & $4.27 \mathrm{E}-02$ & $2.728 \mathrm{E}-02$ & $7.70 \mathrm{E}-01$ \\
\hline 63 & $1.54 \mathrm{E}-02$ & $4.09 \mathrm{E}-02$ & $2.623 \mathrm{E}-02$ & $7.89 \mathrm{E}-01$ \\
\hline 64 & $1.54 \mathrm{E}-02$ & $3.91 \mathrm{E}-02$ & $2.524 \mathrm{E}-02$ & $8.09 \mathrm{E}-01$ \\
\hline 65 & $1.54 \mathrm{E}-02$ & $3.74 \mathrm{E}-02$ & $2.431 \mathrm{E}-02$ & $8.29 \mathrm{E}-01$ \\
\hline 66 & $1.54 \mathrm{E}-02$ & $3.56 \mathrm{E}-02$ & $2.343 \mathrm{E}-02$ & $8.49 \mathrm{E}-01$ \\
\hline 67 & $1.54 \mathrm{E}-02$ & $3.38 \mathrm{E}-02$ & $2.260 \mathrm{E}-02$ & $8.70 \mathrm{E}-01$ \\
\hline 68 & $1.54 \mathrm{E}-02$ & $3.20 \mathrm{E}-02$ & $2.182 \mathrm{E}-02$ & $8.91 \mathrm{E}-01$ \\
\hline 69 & $1.54 \mathrm{E}-02$ & $3.02 \mathrm{E}-02$ & $2.108 \mathrm{E}-02$ & $9.12 \mathrm{E}-01$ \\
\hline 70 & $1.54 \mathrm{E}-02$ & $2.84 \mathrm{E}-02$ & $2.037 \mathrm{E}-02$ & $9.34 \mathrm{E}-01$ \\
\hline 71 & $1.54 \mathrm{E}-02$ & $2.65 \mathrm{E}-02$ & $1.971 \mathrm{E}-02$ & $9.56 \mathrm{E}-01$ \\
\hline 72 & $1.54 \mathrm{E}-02$ & $2.45 \mathrm{E}-02$ & $1.907 \mathrm{E}-02$ & $9.78 \mathrm{E}-01$ \\
\hline 73 & $1.54 \mathrm{E}-02$ & $2.25 \mathrm{E}-02$ & $1.847 \mathrm{E}-02$ & $1.00 \mathrm{E}+00$ \\
\hline 74 & $1.54 \mathrm{E}-02$ & $2.04 \mathrm{E}-02$ & $1.790 \mathrm{E}-02$ & $1.03 \mathrm{E}+00$ \\
\hline 75 & $1.54 \mathrm{E}-02$ & $1.81 \mathrm{E}-02$ & $1.736 \mathrm{E}-02$ & $1.05 \mathrm{E}+00$ \\
\hline 76 & $1.54 \mathrm{E}-02$ & $1.56 \mathrm{E}-02$ & $1.684 \mathrm{E}-02$ & $1.08 \mathrm{E}+00$ \\
\hline 77 & $1.54 \mathrm{E}-02$ & $1.28 \mathrm{E}-02$ & $1.636 \mathrm{E}-02$ & $1.10 \mathrm{E}+00$ \\
\hline 78 & $1.54 \mathrm{E}-02$ & $9.36 \mathrm{E}-03$ & $1.589 \mathrm{E}-02$ & $1.14 \mathrm{E}+00$ \\
\hline 79 & $1.54 \mathrm{E}-02$ & $4.08 \mathrm{E}-03$ & $1.546 \mathrm{E}-02$ & $1.18 \mathrm{E}+00$ \\
\hline
\end{tabular}


Table 7.18. 3000 SCCM

\begin{tabular}{|c|c|c|c|c|}
\hline $\begin{array}{c}d_{p} \\
\mu m\end{array}$ & $\begin{array}{c}\mathbf{h}_{\mathbf{s}} \\
\mathbf{c m}\end{array}$ & $\begin{array}{c}\mathbf{V}_{1}\left(\mathbf{t}_{\mathbf{s}}\right) \\
\mathbf{m} / \mathbf{s}\end{array}$ & $\begin{array}{c}\text { Max. Height } \\
\text { cm }\end{array}$ & $\mathbf{R e}_{p}$ \\
\hline 54 & $4.61 \mathrm{E}-02$ & $2.18 \mathrm{E}-01$ & $1.448 \mathrm{E}+00$ & $1.70 \mathrm{E}+00$ \\
\hline 55 & $4.61 \mathrm{E}-02$ & $2.13 \mathrm{E}-01$ & $1.147 \mathrm{E}+00$ & $1.75 \mathrm{E}+00$ \\
\hline 56 & $4.61 \mathrm{E}-02$ & $2.09 \mathrm{E}-01$ & $9.696 \mathrm{E}-01$ & $1.79 \mathrm{E}+00$ \\
\hline 57 & $4.61 \mathrm{E}-02$ & $2.05 \mathrm{E}-01$ & 8.439E-01 & $1.84 \mathrm{E}+00$ \\
\hline 58 & $4.61 \mathrm{E}-02$ & $2.00 \mathrm{E}-01$ & 7.479E-01 & $1.89 \mathrm{E}+00$ \\
\hline 59 & $4.61 \mathrm{E}-02$ & $1.96 \mathrm{E}-01$ & $6.713 \mathrm{E}-01$ & $1.94 \mathrm{E}+00$ \\
\hline 60 & $4.61 \mathrm{E}-02$ & $1.92 \mathrm{E}-01$ & $6.085 \mathrm{E}-01$ & $1.99 \mathrm{E}+00$ \\
\hline 61 & $4.61 \mathrm{E}-02$ & $1.88 \mathrm{E}-01$ & 5.559E-01 & $2.03 \mathrm{E}+00$ \\
\hline 62 & $4.61 \mathrm{E}-02$ & $1.85 \mathrm{E}-01$ & $5.111 \mathrm{E}-01$ & $2.08 \mathrm{E}+00$ \\
\hline 63 & $4.61 \mathrm{E}-02$ & $1.81 \mathrm{E}-01$ & $4.725 \mathrm{E}-01$ & $2.13 \mathrm{E}+00$ \\
\hline 64 & $4.61 \mathrm{E}-02$ & $1.77 \mathrm{E}-01$ & $4.388 \mathrm{E}-01$ & $2.18 \mathrm{E}+00$ \\
\hline 65 & $4.61 \mathrm{E}-02$ & $1.74 \mathrm{E}-01$ & $4.092 \mathrm{E}-01$ & $2.23 \mathrm{E}+00$ \\
\hline 66 & $4.61 \mathrm{E}-02$ & $1.71 \mathrm{E}-01$ & 3.829E-01 & $2.28 \mathrm{E}+00$ \\
\hline 67 & $4.61 \mathrm{E}-02$ & $1.67 \mathrm{E}-01$ & $3.595 \mathrm{E}-01$ & $2.33 \mathrm{E}+00$ \\
\hline 68 & 4.61E-02 & $1.64 \mathrm{E}-01$ & 3.384E-01 & $2.37 \mathrm{E}+00$ \\
\hline 69 & $4.61 \mathrm{E}-02$ & $1.61 \mathrm{E}-01$ & 3.194E-01 & $2.42 \mathrm{E}+00$ \\
\hline 70 & $4.61 \mathrm{E}-02$ & $1.58 \mathrm{E}-01$ & $3.022 \mathrm{E}-01$ & $2.47 \mathrm{E}+00$ \\
\hline 71 & $4.61 \mathrm{E}-02$ & $1.55 \mathrm{E}-01$ & & $2.52 \mathrm{E}+00$ \\
\hline 72 & $4.61 \mathrm{E}-02$ & $1.52 \mathrm{E}-01$ & $2.721 \mathrm{E}-01$ & $2.57 \mathrm{E}+00$ \\
\hline 73 & 4.61E-02 & 1.49E-01 & $2.589 \mathrm{E}-01$ & $2.62 \mathrm{E}+00$ \\
\hline 74 & $4.61 \mathrm{E}-02$ & $1.46 \mathrm{E}-01$ & $2.467 \mathrm{E}-01$ & $2.67 \mathrm{E}+00$ \\
\hline 75 & $4.61 \mathrm{E}-02$ & $1.44 \mathrm{E}-01$ & $2.355 \mathrm{E}-01$ & $2.72 \mathrm{E}+00$ \\
\hline 76 & $4.61 \mathrm{E}-02$ & $1.41 \mathrm{E}-01$ & $2.252 \mathrm{E}-01$ & $2.77 \mathrm{E}+00$ \\
\hline 77 & 4.61E-02 & $1.38 \mathrm{E}-01$ & $2.155 \mathrm{E}-01$ & $2.82 \mathrm{E}+00$ \\
\hline 78 & $4.61 \mathrm{E}-02$ & $1.36 \mathrm{E}-01$ & $2.066 \mathrm{E}-01$ & $2.87 \mathrm{E}+00$ \\
\hline 79 & $4.61 \mathrm{E}-02$ & $1.33 \mathrm{E}-01$ & $1.982 \mathrm{E}-01$ & $2.92 \mathrm{E}+00$ \\
\hline 80 & $4.61 \mathrm{E}-02$ & $1.31 \mathrm{E}-01$ & $1.904 \mathrm{E}-01$ & $2.97 \mathrm{E}+00$ \\
\hline 81 & 4.61E-02 & $1.28 \mathrm{E}-01$ & $1.831 \mathrm{E}-01$ & $3.02 \mathrm{E}+00$ \\
\hline 82 & 4.61E-02 & $1.26 \mathrm{E}-01$ & $1.762 \mathrm{E}-01$ & $3.06 \mathrm{E}+00$ \\
\hline 83 & $4.61 \mathrm{E}-02$ & $1.24 \mathrm{E}-01$ & $1.697 \mathrm{E}-01$ & $3.11 \mathrm{E}+00$ \\
\hline 84 & 4.61E-02 & $1.21 \mathrm{E}-01$ & $1.637 \mathrm{E}-01$ & $3.17 \mathrm{E}+00$ \\
\hline 85 & 4.61E-02 & 1.19E-01 & $1.579 \mathrm{E}-01$ & $3.22 \mathrm{E}+00$ \\
\hline 86 & 4.61E-02 & $1.17 \mathrm{E}-01$ & $1.525 \mathrm{E}-01$ & $3.27 \mathrm{E}+00$ \\
\hline 87 & $4.61 \mathrm{E}-02$ & $1.14 \mathrm{E}-01$ & $1.474 \mathrm{E}-01$ & $3.32 \mathrm{E}+00$ \\
\hline 88 & $4.61 \mathrm{E}-02$ & $1.12 \mathrm{E}-01$ & $1.426 \mathrm{E}-01$ & $3.37 \mathrm{E}+00$ \\
\hline 89 & $4.61 \mathrm{E}-02$ & $1.10 \mathrm{E}-01$ & $1.380 \mathrm{E}-01$ & $3.42 \mathrm{E}+00$ \\
\hline 90 & $4.61 \mathrm{E}-02$ & $1.08 \mathrm{E}-01$ & $1.337 \mathrm{E}-01$ & $3.47 \mathrm{E}+00$ \\
\hline 91 & $4.61 \mathrm{E}-02$ & $1.06 \mathrm{E}-01$ & $1.296 \mathrm{E}-01$ & $3.52 \mathrm{E}+00$ \\
\hline 92 & 4.61E-02 & $1.04 \mathrm{E}-01$ & $1.256 \mathrm{E}-01$ & $3.57 \mathrm{E}+00$ \\
\hline 93 & 4.61E-02 & $1.02 \mathrm{E}-01$ & $1.219 \mathrm{E}-01$ & $3.62 \mathrm{E}+00$ \\
\hline 94 & $4.61 \mathrm{E}-02$ & $9.98 \mathrm{E}-02$ & $1.183 \mathrm{E}-01$ & $3.67 \mathrm{E}+00$ \\
\hline 95 & $4.61 \mathrm{E}-02$ & $9.79 \mathrm{E}-02$ & $1.150 \mathrm{E}-01$ & $3.72 \mathrm{E}+00$ \\
\hline
\end{tabular}


Table 7.18. 3000 SCCM

\begin{tabular}{|c|c|c|c|c|}
\hline $\begin{array}{c}d_{p} \\
\mu \mathbf{m}\end{array}$ & $\begin{array}{l}\mathbf{h}_{\mathrm{s}} \\
\mathbf{c m}\end{array}$ & $\begin{array}{c}\mathrm{V}_{1}\left(\mathbf{t}_{\mathrm{s}}\right) \\
\mathrm{m} / \mathrm{s}\end{array}$ & $\begin{array}{c}\text { Max. Height } \\
\text { cm }\end{array}$ & $\mathbf{R e}_{\mathrm{p}}$ \\
\hline 96 & $4.61 \mathrm{E}-02$ & $9.59 \mathrm{E}-02$ & $1.117 \mathrm{E}-01$ & $3.77 \mathrm{E}+00$ \\
\hline 97 & $4.61 \mathrm{E}-02$ & $9.40 \mathrm{E}-02$ & $1.086 \mathrm{E}-01$ & $3.83 \mathrm{E}+00$ \\
\hline 98 & $4.61 \mathrm{E}-02$ & $9.20 \mathrm{E}-02$ & $1.057 \mathrm{E}-01$ & $3.88 \mathrm{E}+00$ \\
\hline 99 & $4.61 \mathrm{E}-02$ & $9.01 \mathrm{E}-02$ & $1.028 \mathrm{E}-01$ & $3.93 \mathrm{E}+00$ \\
\hline 100 & $4.61 \mathrm{E}-02$ & $8.82 \mathrm{E}-02$ & $1.001 \mathrm{E}-01$ & $3.98 \mathrm{E}+00$ \\
\hline 101 & $4.61 \mathrm{E}-02$ & $8.64 \mathrm{E}-02$ & $9.752 \mathrm{E}-02$ & $4.03 \mathrm{E}+00$ \\
\hline 102 & $4.61 \mathrm{E}-02$ & $8.45 \mathrm{E}-02$ & $9.503 \mathrm{E}-02$ & $4.08 \mathrm{E}+00$ \\
\hline 103 & $4.61 \mathrm{E}-02$ & $8.27 \mathrm{E}-02$ & $9.264 \mathrm{E}-02$ & $4.14 \mathrm{E}+00$ \\
\hline 104 & $4.61 \mathrm{E}-02$ & $8.08 \mathrm{E}-02$ & $9.034 \mathrm{E}-02$ & $4.19 \mathrm{E}+00$ \\
\hline 105 & $4.61 \mathrm{E}-02$ & $7.90 \mathrm{E}-02$ & $8.813 \mathrm{E}-02$ & $4.24 \mathrm{E}+00$ \\
\hline 106 & $4.61 \mathrm{E}-02$ & 7.72E-02 & $8.601 \mathrm{E}-02$ & $4.29 \mathrm{E}+00$ \\
\hline 107 & 4.61E-02 & 7.54E-02 & $8.397 \mathrm{E}-02$ & $4.35 \mathrm{E}+00$ \\
\hline 108 & $4.61 \mathrm{E}-02$ & $7.36 \mathrm{E}-02$ & $8.201 \mathrm{E}-02$ & $4.40 \mathrm{E}+00$ \\
\hline 109 & $4.61 \mathrm{E}-02$ & $7.18 \mathrm{E}-02$ & $8.011 \mathrm{E}-02$ & $4.45 \mathrm{E}+00$ \\
\hline 110 & $4.61 \mathrm{E}-02$ & $7.00 \mathrm{E}-02$ & 7.829E-02 & $4.51 \mathrm{E}+00$ \\
\hline 111 & $4.61 \mathrm{E}-02$ & $6.82 \mathrm{E}-02$ & 7.653E-02 & $4.56 \mathrm{E}+00$ \\
\hline 112 & $4.61 \mathrm{E}-02$ & $6.65 \mathrm{E}-02$ & 7.483E-02 & $4.62 \mathrm{E}+00$ \\
\hline 113 & $4.61 \mathrm{E}-02$ & $6.47 \mathrm{E}-02$ & $7.320 \mathrm{E}$ & $4.67 \mathrm{E}+00$ \\
\hline 114 & $4.61 \mathrm{E}-02$ & $6.29 \mathrm{E}-02$ & 7.161E-02 & $4.72 \mathrm{E}+00$ \\
\hline 115 & $4.61 \mathrm{E}-02$ & $6.11 \mathrm{E}-02$ & 7.009E-02 & $4.78 \mathrm{E}+00$ \\
\hline 116 & $4.61 \mathrm{E}-02$ & $5.93 \mathrm{E}-02$ & $6.861 \mathrm{E}-02$ & $4.83 \mathrm{E}+00$ \\
\hline 117 & $4.61 \mathrm{E}-02$ & $5.75 \mathrm{E}-02$ & $6.718 \mathrm{E}-02$ & $4.89 \mathrm{E}+00$ \\
\hline 118 & $4.61 \mathrm{E}-02$ & $5.57 \mathrm{E}-02$ & $6.580 \mathrm{E}-02$ & $4.94 \mathrm{E}+00$ \\
\hline 119 & $4.61 \mathrm{E}-02$ & 5.39E-02 & $6.446 \mathrm{E}-02$ & $5.00 \mathrm{E}+00$ \\
\hline 120 & 4.61E-02 & $5.20 \mathrm{E}-02$ & $6.317 \mathrm{E}-02$ & $5.06 \mathrm{E}+00$ \\
\hline 121 & $4.61 \mathrm{E}-02$ & $5.02 \mathrm{E}-02$ & $6.192 \mathrm{E}-02$ & $5.11 \mathrm{E}+00$ \\
\hline 122 & $4.61 \mathrm{E}-02$ & $4.83 \mathrm{E}-02$ & $6.070 \mathrm{E}-02$ & $5.17 \mathrm{E}+00$ \\
\hline 123 & $4.61 \mathrm{E}-02$ & $4.64 \mathrm{E}-02$ & $5.953 \mathrm{E}-02$ & $5.23 \mathrm{E}+00$ \\
\hline 124 & $4.61 \mathrm{E}-02$ & $4.45 \mathrm{E}-02$ & $5.839 \mathrm{E}-02$ & $5.29 \mathrm{E}+00$ \\
\hline 125 & $4.61 \mathrm{E}-02$ & $4.25 \mathrm{E}-02$ & $5.728 \mathrm{E}-02$ & $5.34 \mathrm{E}+00$ \\
\hline 126 & $4.61 \mathrm{E}-02$ & $4.04 \mathrm{E}-02$ & $5.621 \mathrm{E}-02$ & $5.40 \mathrm{E}+00$ \\
\hline 127 & 4.61E-02 & $3.84 \mathrm{E}-02$ & $5.516 \mathrm{E}-02$ & $5.46 \mathrm{E}+00$ \\
\hline 128 & $4.61 \mathrm{E}-02$ & $3.62 \mathrm{E}-02$ & $5.415 \mathrm{E}-02$ & $5.52 \mathrm{E}+00$ \\
\hline 129 & $4.61 \mathrm{E}-02$ & $3.40 \mathrm{E}-02$ & $5.317 \mathrm{E}-02$ & $5.59 \mathrm{E}+00$ \\
\hline 130 & $4.61 \mathrm{E}-02$ & $3.17 \mathrm{E}-02$ & $5.222 \mathrm{E}-02$ & $5.65 \mathrm{E}+00$ \\
\hline 131 & $4.61 \mathrm{E}-02$ & $2.92 \mathrm{E}-02$ & $5.129 \mathrm{E}-02$ & $5.71 \mathrm{E}+00$ \\
\hline 132 & $4.61 \mathrm{E}-02$ & $2.66 \mathrm{E}-02$ & $5.040 \mathrm{E}-02$ & $5.78 \mathrm{E}+00$ \\
\hline 133 & $4.61 \mathrm{E}-02$ & $2.38 \mathrm{E}-02$ & $4.952 \mathrm{E}-02$ & $5.85 \mathrm{E}+00$ \\
\hline 134 & $4.61 \mathrm{E}-02$ & $2.07 \mathrm{E}-02$ & $4.868 \mathrm{E}-02$ & $5.92 \mathrm{E}+00$ \\
\hline 135 & $4.61 \mathrm{E}-02$ & $1.71 \mathrm{E}-02$ & $4.786 \mathrm{E}-02$ & $5.99 \mathrm{E}+00$ \\
\hline 136 & $4.61 \mathrm{E}-02$ & $1.27 \mathrm{E}-02$ & 4.707E-02 & $6.08 \mathrm{E}+00$ \\
\hline 137 & $4.61 \mathrm{E}-02$ & $6.02 \mathrm{E}-03$ & $4.631 \mathrm{E}-02$ & $6.18 \mathrm{E}+00$ \\
\hline
\end{tabular}




\subsection{Conclusions}

Table 7.8 through Table 7.18 demonstrate that even for a flow rate of 3000 SCCM and a value of $h_{s}$ that is an order of magnitude larger than that predicted by Eq. (7.3), no particles larger than would be expected to be ejected due to superficial flow will be expected to be ejected due to excess momentum imparted by post-frit velocity flow. However, in the development of the models used in this chapter, it was always assumed that the particle motion could be described using the drag force associated with the Stokes flow regime, which is only valid for a particle Reynolds number less than 1. Unfortunately, the particle Reynolds numbers for a few of the particles in the 1000-SCCM case and all of the particle Reynold numbers in the 3000-SCCM case are greater than 1. This indicates that some particle Reynolds numbers for flows between 1000 and 3000 SCCM will also be greater than one. The reason that the drag associated with particle motion in the Stokes regime was assumed is that it is simple to implement whereas particle motion outside the Stokes regime is more complicated. It is important to quantify the effect of using the drag from Stokes flow in this chapter. Scoping studies indicate that if corrections are made to the model in this chapter for particle motion above the Stokes regime, the largest particles to be lifted from the sample boat would be 61 micrometers in diameter instead of 53 micrometers. Adjustments for lower flow rates are less noticeable.

The model of particle motion within the boat that is presented in this chapter should be sufficient to dispel the concern that the increase in flow immediately above the frit causes a noticeable increase in the fraction of particles suspended from the sample boat. Even if the mixing height $\left(\mathrm{h}_{\mathrm{s}}\right)$ is assumed to be a whole order of magnitude above the value calculated with the method indicated at the beginning of this chapter, and the effects of particle flow outside the Stokes Regime are considered, the increase in the largest particle size to be lifted from the sample boat is only slightly relevant for the highest flow rates considered in this study. In conclusion, the model presented in this chapter should be an accurate depiction of ideal particle motion within the test system and systems analogous to it. Deviation of particle behavior from that described in this chapter is most likely due to phenomena such as sticking and agglomeration. Both of these phenomena would result in lower particle releases than those predicted in this chapter. 


\subsection{References}

Anderson BL, RW Carlson, and LE Fischer. 1996. Containment Analysis for Type B Packages Used to Transport Various Contents. NUREG/CR-6487, Lawrence Livermore National Laboratory, Livermore, CA.

ASTM International C776-00 Standard Specification for Sintered Uranium Dioxide Pellets.

Bernard LC, JL Jacoud and P Vesco. 2002. "An efficient model for the analysis of fission gas release." Journal of Nuclear Materials 302:125-134.

Bechtel SAIC Company (BSC). 2007a. Release Fractions for Spent Nuclear Fuel and HighLevel Waste. 000-00C-MGR0-03400-000-00A, Las Vegas, Nevada.

Bechtel SAIC Company (BSC). 2007b. Characteristics for the Representative Commercial Spent Fuel Assembly for Preclosure Normal Operations. 000-PSA-MGR0-00700-000-00A, Las Vegas, Nevada.

Civilian Radioactive Waste Management Service-Management and Operations (CRWMS M\&O). 2000. "Clad Degradation - Dry Unzipping." ANL-EBS-MD-000013, Rev 00, Las Vegas, Nevada.

Colle JY, J-P Hiernaut, D Papaioannou, C Ronchi, and A Sasahara. 2006. "Fission product release in high-burnup $\mathrm{UO}_{2}$ oxidized to $\mathrm{U}_{3} \mathrm{O}_{8}$." Journal of Nuclear Materials 348:229-242.

Davis PR, DL Strenge, and J Mishima. 1998. Accident Analysis for Continued Storage. Jason Technologies, Las Vegas, Nevada.

Deen WM. 1998. "Diffusive Fluxes and Material Properties" (Chapter 1). Analysis of Transport Phenomena. Oxford University Press, New York.

DOE (see U.S. Department of Energy)

Einziger RE, and C Beyer. 2007. "Characteristics and Behavior of High Burnup Fuel that May Affect the Source Terms for Cask Accidents." U.S. Nuclear Regulatory Commission/PNNL Preprint, submitted to Nuclear Technology.

Einziger RE, and RV Strain. 1986. "Behavior of Breached Pressurized Water Reactor SpentFuel Rods in an Air Atmosphere Between 250 and 360 ${ }^{\circ}$ C." Nuclear Technology 75:82-95.

Electric Power Research Institute (EPRI). 1986. Oxidation of Spent Fuel Between 250 and $360^{\circ} \mathrm{C}$. EPRI NP-4524, Palo Alto, California.

Ferry C, C Poinsot, V Broudic, C Cappelaere, L Desgranges, P Garcia, C Jegou, P Lovera, P Marimbeau, JP Piron, A Poulesquen, D Roudil, JM Gras, and P Bouffioux. 2005. Synthesis on the Spent Fuel Long Term Evolution. CEA-R-6084, Commissariat à l'Énergie Atomique (CEA), Gif-Sur-Yvette Cedex, France. 
Guenther RJ, DE Blahnik, TK Campbell, UP Jenquin, and J Mendel. 1988a. Characterization of Spent Fuel Approved Testing Material - ATM-103. PNL-5109-103, Pacific Northwest

Laboratory, Richland, Washington.

Guenther RJ, DE Blahnik, TK Campbell, UP Jenquin, JE Mendel, and CK Thornhill. 1988b. Characterization of Spent Fuel Approved Testing Material -ATM-106. PNL-5109-106, Pacific Northwest Laboratory, Richland, Washington.

Guenther RJ, DE Blahnik, UP Jenquin, JE Mendel, LE Thomas, and CK Thornhill. 1991a. Characterization of Spent Fuel Approved Testing Material - ATM-104. PNL-5109-104, Pacific Northwest Laboratory, Richland, Washington.

Guenther RJ, DE Blahnik, TK Campbell, UP Jenquin, and JE Mendel. 1991b. Characterization of Spent Fuel Approved Testing Material - ATM-105. PNL-5109-105, Pacific Northwest Laboratory, Richland, Washington.

Guenther RJ, DE Blahnik, and NJ Wildung. 1994. Radiochemical Analyses of Several Spent Fuel Approved Testing Materials. PNL-10113, Pacific Northwest Laboratory, Richland, Washington.

Hanson BD. 1998. The Burnup Dependence of Light Water Reactor Spent Fuel Oxidation. PNL-11929, Pacific Northwest National Laboratory, Richland, Washington.

Iwasaki M, T Sakurai, N Ishikawa, and Y Kobayashi. 1968. "Oxidation of $\mathrm{UO}_{2}$ Pellets in Air Effect of Heat-Treatment of Pellet on Particle Size Distribution of Powders Produced." J. Nucl. Sci. Technol. 5(12):652.

Jain V, G Cragnolino, and L Howard. 2004. A review report on high burnup spent nuclear fueldisposal issues. CNWRA 2004-08, Center for Nuclear Waste Regulatory Analyses, San Antonio, Texas.

Jernkvist LO, and AR Massih. 2004. Assessment of Core Failure Limits for Light Water Reactor Fuel under Reactivity Initiated Accidents. SKI Report 2005:16, Quantum Technologies AB, Uppsala, Sweden.

Johnson L, C Ferry, C Poinssot, and P Lovera. 2005. "Spent fuel radionuclide source-term model for assessing spent fuel performance in geological disposal. Part I: Assessment of the instant release fraction." Journal of Nuclear Materials 346:56-65.

Kansa EJ, BD Hanson, and RB Stout. 1998. "Grain and burnup dependence of spent fuel oxidation: Geological repository impact." Los Alamos National Laboratory (LLNL) preprint UCRL-JC-131592, Prepared for the Materials Research Society 1998 Fall Meeting, Boston, Massachusetts, November 30-December 4, 1998.

Koo Y-H, B-H Lee, J-S Cheon, and D-S Sohn. 2001. "Pore pressure and swelling in the rim region of LWR high burnup $\mathrm{UO}_{2}$ fuel." Journal of Nuclear Materials 295:213-220. 
Koo Y-H, JY Oh, BH Lee, and DS Sohn. 2003. "Three-dimensional simulation of threshold

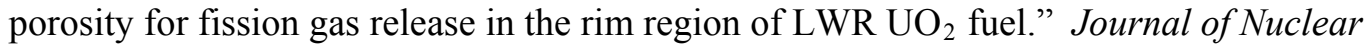
Materials 321:249-255.

Lassmann K, CT Walker, j van der Laar, and F Lindstrom. 1995. "Modeling the High Burnup $\mathrm{UO}_{2}$ Structure in LWR Fuel." Journal of Nuclear Materials 226:1-8.

Liu Z, DS Cox, RD Barrand, and CEL Hunt. 1992. "Particle Size Distributions of $\mathrm{U}_{3} \mathrm{O}_{8}$ Produced by Oxidation in Air at 300-900 ${ }^{\circ}$." In: 13th Annual Conference of the Canadian Nuclear Society, June 7 to 10, 1992. Canadian Nuclear Society, Saint John, New Brunswick, Canada.

Matzke Hj, and J Spino. 1997. "Formation of the rim structure in high burnup fuel." Journal of Nuclear Materials 248:170-179.

McEachern RJ, and P Taylor. 1998. "A review of the oxidation of uranium dioxide at temperatures below $400^{\circ} \mathrm{C}$." Journal of Nuclear Materials 254:87-121.

McKinnon MA, and ME Cunningham. 2003. Dry Storage Demonstration for High-Burnup Spent Nuclear Fuel-Feasibility Study. PNNL-14390, Pacific Northwest National Laboratory, Richland, Washington.

Novak J, IJ Hastings, E Mizzan, and RJ Chenier. 1983. "Postirradiation behavior of $\mathrm{UO}_{2}$ Fuel I: Elements at 220 to $250^{\circ} \mathrm{C}$ in Air." Nuclear Technology 63:254-265.

NRC (see U.S. Nuclear Regulatory Commission)

Spino J, AD Stalios, H Santa Cruz, and D Baron. 2006. "Stereological evolution of the rim structure in PWR-fuels at prolonged irradiation: Dependencies with burn-up and temperature." Journal of Nuclear Materials 354:66-84.

Spino J, K Vennix, and M Coquerelle. 1996. "Detailed Characterisation of the Rim Microstructure in PWR Fuels in the Burn-Up Range 40-67 GWd/MTUM." Journal of Nuclear Materials 231:179-190.

Sprung JL, DJ Ammerman, NL Breivik, RJ Dukart, and FL Kanipe. 2000. "Reexamination of Spent Fuel Shipment Risk Estimates.” NUREG/CR-6672, Vol. 1 (SAND2000-0234), Sandia National Laboratories, Albuquerque, New Mexico.

Une K, M Hirai, K Nogita, T Hosokawa, Y Suzawa, S Shimizu, and Y Etoh. 2000. "Rim structure formation and high burnup fuel behavior of large-grained $\mathrm{UO}_{2}$ fuels." Journal of Nuclear Materials 278:54-63.

Une K, K Nogita, S Kashibe, and M Imamura. 1992. "Microstructural change and its influence on fission gas release in high burnup $\mathrm{UO}_{2}$ fuel." Journal of Nuclear Materials 188:65-72.

U.S. Department of Energy (DOE). 1994. Airborne Release Fractions/Rates and Respirable Fractions for Nonreactor Nuclear Facilities. DOE Handbook, DOE-HDBK-3010-94, Washington, D.C. 
U.S. Nuclear Regulatory Commission (NRC). 2008. Interim Staff Guidance-5, Revision 1. Spent Fuel Project Office (SFPO). Available at: http://www.nrc.gov/reading-rm/doccollections/isg/spent-fuel.html. Accessed 01-17-2008.

Vaidyanathan S, RD Reager, RW Warner, C Martinez, Y Shirai, Y Iwano. 1997. "High Burnup BWR Fuel Pellet Performance.” In: Proc. Int. Topical Meeting on Light Water Reactor Fuel Performance, Portland, Oregon, pp. 471-477.

Walker CT, C Bagger, and M Mogensen. 1996. "Observations on the release of cesium from $\mathrm{UO}_{2}$ fuel." Journal of Nuclear Materials 240:32-42. 


\section{Appendix A}

\section{Mass Release from CSNF Rod Tests}

This appendix is composed of tables containing the particle mass released and the particle mass detected for each CSNF rod test. The column labeled "Detected" represents the mass of particles determined from OPC measurements. The column labeled "Release" represents the mass difference measured in the sample. Particle-size distributions generated from the data collected by the OPC are also presented. 


\section{Appendix A: Mass Release from CSNF Rod Tests}

\begin{tabular}{|c|c|c|c|c|c|c|}
\hline \multicolumn{7}{|c|}{ ATM106 Test 1} \\
\hline Events & Description & Detected [mg] & $\begin{array}{c}\text { Release } \\
{[\mathrm{mg}]}\end{array}$ & $\mathrm{d} 10[\mu \mathrm{m}]$ & $\mathrm{d} 50[\mu \mathrm{m}]$ & $\mathrm{d} 90[\mu \mathrm{m}]$ \\
\hline 9 & Flow controller exchange: FC1 closed, FC2 opened & 3.25E-04 & $5.00 \mathrm{E}-03$ & 1.85 & 5.46 & 10.21 \\
\hline 22 & Housing tap start & 2.30E-04 & $3.53 \mathrm{E}-03$ & 2.53 & 12.81 & 17.72 \\
\hline $27-28$ & V2 pressure isolation pulse & $2.28 \mathrm{E}-03$ & $3.50 \mathrm{E}-02$ & 1.96 & 8.15 & 16.12 \\
\hline 29 & V2 pressure isolation pulse & 2.11E-04 & $3.25 \mathrm{E}-03$ & 1.92 & 5.55 & 13.46 \\
\hline $31-32$ & V2 pressure isolation pulse & 1.53E-03 & 2.36E-02 & 1.82 & 6.96 & 15.90 \\
\hline $33-34$ & V3 pressure isolation pulse & $1.16 \mathrm{E}-03$ & $1.78 \mathrm{E}-02$ & 1.71 & 5.53 & 15.77 \\
\hline 35 & V3 pressure isolation pulse & 1.38E-04 & $2.12 \mathrm{E}-03$ & 1.68 & 6.52 & 13.64 \\
\hline 37 & V3 pressure isolation pulse & 1.03E-04 & 1.59E-03 & 1.89 & 7.25 & 16.40 \\
\hline $39-40$ & V3 pressure isolation pulse & 1.09E-03 & $1.68 \mathrm{E}-02$ & 1.71 & 5.75 & 14.89 \\
\hline 41 & V3 pressure isolation pulse & 2.39E-04 & $3.68 \mathrm{E}-03$ & 1.49 & 3.65 & 9.07 \\
\hline 43 & V3 pressure isolation pulse & $1.87 \mathrm{E}-04$ & $2.87 \mathrm{E}-03$ & 1.53 & 3.47 & 13.59 \\
\hline $45-46$ & Housing tap & $6.64 \mathrm{E}-06$ & $1.02 \mathrm{E}-04$ & 1.06 & 2.27 & 3.25 \\
\hline 48 & V3 pressure isolation pulse & $1.49 \mathrm{E}-03$ & $2.30 \mathrm{E}-02$ & 1.70 & 5.11 & 15.39 \\
\hline 50 & V3 pressure isolation pulse & 4.83E-04 & $7.43 \mathrm{E}-03$ & 1.62 & 3.43 & 10.44 \\
\hline 52 & V3 pressure isolation pulse & $1.53 \mathrm{E}-03$ & $2.35 \mathrm{E}-02$ & 1.62 & 4.36 & 14.25 \\
\hline 54 & V3 pressure isolation pulse & 1.59E-03 & 2.44E-02 & 1.57 & 3.47 & 10.37 \\
\hline 56 & V3 pressure isolation pulse & $2.18 \mathrm{E}-03$ & 3.35E-02 & 1.63 & 3.37 & 10.34 \\
\hline 58 & Increased flow set point from 0.2 to $3.0 \mathrm{SLM}$ & 1.16E-07 & 1.79E-06 & $<0.6$ & 1.07 & 1.41 \\
\hline 60 & V3 pressure isolation pulse & 1.35E-03 & $2.08 \mathrm{E}-02$ & 1.50 & 3.27 & 10.29 \\
\hline $62-63$ & V3 pressure isolation pulse & 1.73E-03 & $2.66 \mathrm{E}-02$ & 1.53 & 3.05 & 10.34 \\
\hline 64 & V3 pressure isolation pulse & 2.74E-04 & $4.21 \mathrm{E}-03$ & 1.31 & 3.20 & 10.51 \\
\hline 66 & V3 pressure isolation pulse & 3.45E-04 & 5.30E-03 & 1.29 & 2.86 & 9.01 \\
\hline 69 & V3 pressure isolation pulse & $5.71 \mathrm{E}-05$ & 8.78E-04 & 1.02 & 2.41 & 5.74 \\
\hline $72-73$ & Housing tap & 2.09E-04 & $3.21 \mathrm{E}-03$ & 3.99 & 10.34 & 16.39 \\
\hline Total & Sum of all non-background release events & 1.87E-02 & 2.88E-01 & 1.65 & 4.46 & 14.45 \\
\hline
\end{tabular}




\begin{tabular}{|c|c|c|c|c|c|c|}
\hline \multicolumn{7}{|c|}{ ATM106 Test 2} \\
\hline Events & Description & Detected [mg] & $\begin{array}{c}\text { Release } \\
{[\mathrm{mg}]}\end{array}$ & $\begin{array}{c}d 10 \\
{[\mu \mathrm{m}]}\end{array}$ & $\begin{array}{c}\mathrm{d50} \\
{[\mu \mathrm{m}]}\end{array}$ & $\begin{array}{r}\mathrm{d90} \\
{[\mu \mathrm{m}]}\end{array}$ \\
\hline 3 & Experiment start; flow set point to $10 \mathrm{SCCM}$ & $1.58 \mathrm{E}-07$ & 2.43E-06 & $<0.6$ & $<0.6$ & 0.92 \\
\hline 4 & Flow set point increased from 10 to $30 \mathrm{SCCM}$ & 1.77E-06 & 2.73E-05 & 0.92 & 1.92 & 3.18 \\
\hline 5 & Flow set point increased from 30 to $50 \mathrm{SCCM}$ & $6.13 \mathrm{E}-05$ & 9.43E-04 & 1.12 & 2.35 & 3.43 \\
\hline 6 & Flow set point increased from 50 to $75 \mathrm{SCCM}$ & $4.41 E-04$ & $6.78 \mathrm{E}-03$ & 1.32 & 2.53 & 5.06 \\
\hline 7 & Flow set point increased from 75 to $100 \mathrm{SCCM}$ & $9.16 \mathrm{E}-04$ & 1.41E-02 & 1.21 & 2.51 & 5.23 \\
\hline 8 & Flow set point increased from 100 to $125 \mathrm{SCCM}$ & $1.08 \mathrm{E}-03$ & 1.66E-02 & 1.14 & 2.41 & 4.24 \\
\hline 9 & Flow set point increased from 125 to $150 \mathrm{SCCM}$ & 2.39E-03 & 3.68E-02 & 1.12 & 2.40 & 4.07 \\
\hline 10 & Flow set point increased from 150 to $200 \mathrm{SCCM}$ & 3.24E-03 & 4.98E-02 & 1.05 & 2.36 & 3.96 \\
\hline 11 & FC change: FC1 stopped, FC2 started & 8.36E-03 & 1.29E-01 & 1.07 & 2.36 & 3.89 \\
\hline $12-13$ & Flow set point increased from 0.2 to $0.3 \mathrm{SLM}$; pressure increased to obtain flow & 1.75E-03 & 2.69E-02 & 0.95 & 2.25 & 3.45 \\
\hline $14-15$ & Flow set point increased from 0.3 to 0.4 SLM; pressure increased to obtain flow & 8.54E-03 & 1.31E-01 & 0.98 & 2.29 & 3.78 \\
\hline $16-17$ & Flow set point increased from 0.4 to 0.5 SLM; pressure increased to obtain flow & 7.61E-03 & 1.17E-01 & 0.92 & 2.21 & 3.45 \\
\hline $18-19$ & Flow set point increased from 0.5 to $0.6 \mathrm{SLM}$; pressure increased to obtain flow & $5.10 \mathrm{E}-03$ & 7.84E-02 & 0.88 & 2.17 & 3.45 \\
\hline $20-21$ & Flow set point increased from 0.6 to 0.7 SLM; pressure increased to obtain flow & $7.82 \mathrm{E}-03$ & 1.20E-01 & 0.88 & 2.18 & 3.48 \\
\hline $22-23$ & Flow set point increased from 0.7 to 0.8 SLM; pressure increased to obtain flow & 4.08E-03 & $6.28 \mathrm{E}-02$ & 0.84 & 2.19 & 4.22 \\
\hline $24-25$ & Flow set point increased from 0.8 to $0.9 \mathrm{SLM}$; pressure increased to obtain flow & $5.21 \mathrm{E}-03$ & 8.01E-02 & 0.80 & 2.16 & 4.09 \\
\hline $26-27$ & Flow set point increased from 0.9 to $1.0 \mathrm{SLM}$; pressure increased to obtain flow & $5.46 \mathrm{E}-03$ & 8.40E-02 & 0.80 & 2.11 & 3.46 \\
\hline $28-29$ & Flow set point increased from 1.0 to $1.5 \mathrm{SLM}$; pressure increased to obtain flow & $1.48 \mathrm{E}-02$ & 2.28E-01 & 0.91 & 2.22 & 3.96 \\
\hline $30-31$ & Flow set point increased from 1.5 to 2.0 SLM; pressure increased to obtain flow & $9.51 \mathrm{E}-03$ & 1.46E-01 & 0.87 & 2.18 & 3.73 \\
\hline $32-33$ & Flow set point increased from 2.0 to 2.5 SLM; pressure increased to obtain flow & $6.71 \mathrm{E}-03$ & 1.03E-01 & 0.81 & 2.19 & 5.02 \\
\hline $34-35$ & Flow set point increased from 2.5 to 3.0 SLM; pressure increased to obtain flow & 4.68E-03 & $7.20 \mathrm{E}-02$ & 0.76 & 2.12 & 4.00 \\
\hline $36-37$ & Flow set point increased from 3 to 9 SLM; pressure increased to obtain flow & 2.07E-02 & 3.19E-01 & 0.82 & 2.18 & 5.28 \\
\hline 38 & Decrease flow set point from 9 to 3 SLM & 3.15E-05 & 4.85E-04 & 0.73 & 2.09 & 3.68 \\
\hline $39-40$ & V3 pressure isolation pulse & 8.59E-04 & $1.32 \mathrm{E}-02$ & 0.78 & 2.06 & 3.47 \\
\hline $41-42$ & V3 pressure isolation pulse & 4.44E-04 & $6.83 \mathrm{E}-03$ & 0.76 & 2.07 & 3.50 \\
\hline 43 & Accidentally hit something in cell, causing a small vibration. & 1.38E-05 & 2.12E-04 & $<0.6$ & 2.01 & 4.98 \\
\hline 44 & Intentionally hit the floor of the hot cell, causing a small vibration. & 1.35E-04 & 2.07E-03 & 0.87 & 2.25 & 3.95 \\
\hline $45-46$ & Housing tap & 1.37E-02 & 2.11E-01 & 0.94 & 2.22 & 3.49 \\
\hline $47-48$ & Housing tap & 3.00E-03 & 4.62E-02 & 0.85 & 2.16 & 3.46 \\
\hline Total & Sum of all non-background release events & 1.37E-01 & $2.10 \mathrm{E}+00$ & 0.90 & 2.22 & 3.93 \\
\hline
\end{tabular}




\begin{tabular}{|c|c|c|c|c|c|c|}
\hline \multicolumn{7}{|c|}{ ATM106 Test 3} \\
\hline Events & Description & Detected $[\mathrm{mg}]$ & $\begin{array}{c}\text { Release } \\
\text { [mg] }\end{array}$ & $\begin{array}{c}\mathrm{d} 10 \\
{[\mu \mathrm{m}]}\end{array}$ & $\begin{array}{c}\mathrm{d50} \\
{[\mu \mathrm{m}]}\end{array}$ & $\begin{array}{c}d 90 \\
{[\mu \mathrm{m}]}\end{array}$ \\
\hline 2 & Increased flow set point from 10 to $30 \mathrm{SCCM}$ & $2.48 \mathrm{E}-06$ & 3.81E-05 & 1.51 & 2.40 & 3.28 \\
\hline 3 & Increased flow set point from 30 to $50 \mathrm{SCCM}$ & $5.54 \mathrm{E}-06$ & $8.52 E-05$ & 0.93 & 2.05 & 3.21 \\
\hline 4 & Increased flow set point from 50 to $75 \mathrm{SCCM}$ & $3.35 \mathrm{E}-05$ & $5.15 E-04$ & 1.02 & 2.20 & 3.24 \\
\hline 5 & Increased flow set point from 75 to 100 SCCM & 2.91E-05 & 4.47E-04 & 0.99 & 2.17 & 3.23 \\
\hline 6 & Increased flow set point from 100 to $125 \mathrm{SCCM}$ & 1.04E-04 & 1.60E-03 & 1.01 & 2.47 & 9.64 \\
\hline 7 & Increased flow set point from 125 to $150 \mathrm{SCCM}$ & $1.18 \mathrm{E}-04$ & 1.82E-03 & 1.06 & 2.57 & 12.58 \\
\hline 8 & Increased flow set point from 150 to $200 \mathrm{SCCM}$ & $3.61 \mathrm{E}-04$ & $5.55 \mathrm{E}-03$ & 0.92 & 2.19 & 3.43 \\
\hline 9 & Flow controller exchange: FC1 to FC2 & 4.03E-03 & $6.21 \mathrm{E}-02$ & 0.90 & 2.19 & 3.49 \\
\hline 10 & Increased flow set point from 200 to 300 SCCM & $1.48 \mathrm{E}-06$ & 2.28E-05 & 0.61 & 1.85 & 3.17 \\
\hline 11 & Increased flow set point from 300 to $400 \mathrm{SCCM}$ & $3.12 \mathrm{E}-06$ & 4.80E-05 & 0.68 & 1.76 & 3.15 \\
\hline $12-13$ & Increased flow set point from 400 to 500 SCCM; increased pressure to obtain flow & $2.63 \mathrm{E}-04$ & 4.05E-03 & 0.68 & 2.01 & 4.33 \\
\hline $14-15$ & Increased flow set point from 500 to 600 SCCM; increased pressure to obtain flow & $1.21 \mathrm{E}-03$ & $1.86 \mathrm{E}-02$ & 0.69 & 1.96 & 3.41 \\
\hline $16-17$ & Increased flow set point from 600 to $700 \mathrm{SCCM}$; increased pressure to obtain flow & $2.02 \mathrm{E}-03$ & 3.11E-02 & 0.68 & 1.97 & 3.48 \\
\hline $18-19$ & Increased flow set point from 700 to $800 \mathrm{SCCM}$; increased pressure to obtain flow & $2.41 \mathrm{E}-03$ & $3.71 \mathrm{E}-02$ & 0.66 & 1.93 & 3.47 \\
\hline $20-21$ & Increased flow set point from 800 to $900 \mathrm{SCCM}$; increased pressure to obtain flow & $2.37 \mathrm{E}-03$ & $3.65 \mathrm{E}-02$ & 0.85 & 2.81 & 12.91 \\
\hline $22-23$ & Increased flow set point from 900 to 1000 SCCM; increased pressure to obtain flow & $1.46 \mathrm{E}-03$ & $2.25 \mathrm{E}-02$ & 0.68 & 2.10 & 7.15 \\
\hline $24-25$ & Increased flow set point from 1.0 to $1.5 \mathrm{SLM}$; increased pressure to obtain flow & $8.02 \mathrm{E}-03$ & $1.23 E-01$ & 0.74 & 2.02 & 3.70 \\
\hline $26-27$ & Increased flow set point from 1.5 to 2.0 SLM; increased pressure to obtain flow & $5.46 \mathrm{E}-03$ & 8.40E-02 & 0.71 & 1.98 & 4.29 \\
\hline $28-29$ & Increased flow set point from 2.0 to $2.5 \mathrm{SLM}$; increased pressure to obtain flow & 3.06E-03 & 4.71E-02 & 0.65 & 2.13 & 8.11 \\
\hline $30-31$ & Increased flow set point from 2.5 to $3.0 \mathrm{SLM}$; increased pressure to obtain flow & 3.59E-03 & $5.52 \mathrm{E}-02$ & 0.67 & 2.05 & 7.95 \\
\hline $32-33$ & Increased flow set point from 3 to 9 SLM; increased pressure to obtain flow & $1.46 \mathrm{E}-02$ & $2.24 \mathrm{E}-01$ & 0.72 & 2.22 & 9.12 \\
\hline 34 & Decreased flow set point from 9 to 3 SLM & $2.03 E-05$ & $3.12 \mathrm{E}-04$ & 0.84 & 2.91 & 8.82 \\
\hline 35 & Closed V3 (residual particles) & 1.19E-05 & 1.83E-04 & 0.84 & 2.14 & 3.23 \\
\hline $36-37$ & V3 pressure isolation pulse & $1.50 \mathrm{E}-04$ & $2.31 \mathrm{E}-03$ & $<0.6$ & 1.78 & 3.67 \\
\hline 38 & V3 pressure isolation pulse & 1.05E-04 & 1.61E-03 & 0.61 & 2.04 & 8.03 \\
\hline 40 & V3 pressure isolation pulse & $9.34 \mathrm{E}-05$ & 1.44E-03 & $<0.6$ & 1.97 & 6.19 \\
\hline 41 & Shook the line leading from the sample chamber to the OPC & $3.09 E-07$ & 4.76E-06 & 0.67 & 2.12 & 3.22 \\
\hline 42 & Dropped a wrench on the cell floor (causing a small vibration) & 4.22E-04 & $6.50 \mathrm{E}-03$ & 0.77 & 2.09 & 3.63 \\
\hline 43-44 & Housing tap & $2.08 \mathrm{E}-02$ & 3.21E-01 & 1.00 & 2.44 & 7.76 \\
\hline $45-46$ & Housing tap & $9.08 \mathrm{E}-03$ & $1.40 \mathrm{E}-01$ & 0.93 & 2.38 & 7.27 \\
\hline Total & Sum of all non-background release events & $7.98 \mathrm{E}-02$ & $1.23 \mathrm{E}+00$ & 0.81 & 2.24 & 6.93 \\
\hline
\end{tabular}




\begin{tabular}{|c|c|c|c|c|c|c|}
\hline \multicolumn{7}{|c|}{ HBU 539B3 Test 1} \\
\hline Events & Description & Detected [mg] & Release [mg] & $\mathrm{d} 10[\mu \mathrm{m}]$ & $\mathrm{d} 50[\mu \mathrm{m}]$ & d90 [um] \\
\hline 3 & Increased flow set point from 50 to $100 \mathrm{SCCM}$; increased pressure to obtain flow & $2.11 \mathrm{E}-05$ & $3.24 \mathrm{E}-04$ & 0.96 & 2.10 & 3.22 \\
\hline 4 & Increased flow set point from 100 to $150 \mathrm{SCCM}$; increased pressure to obtain flow & $2.39 \mathrm{E}-05$ & $3.68 \mathrm{E}-04$ & 0.96 & 2.13 & 3.23 \\
\hline 5 & Increased flow set point from 150 to $200 \mathrm{SCCM}$; increased pressure to obtain flow & $2.97 \mathrm{E}-05$ & 4.56E-04 & 0.89 & 2.09 & 3.22 \\
\hline 9 & Increased flow set point from 0 to $200 \mathrm{SCCM}$ & $1.65 \mathrm{E}-06$ & 2.53E-05 & 0.79 & 2.03 & 3.21 \\
\hline 10 & Increased flow set point from 200 to $300 \mathrm{SCCM}$ & 1.53E-04 & $2.36 \mathrm{E}-03$ & 1.19 & 3.53 & 16.11 \\
\hline 11 & Increased flow set point from 300 to 400 SCCM; increased pressure to obtain flow & 1.05E-04 & $1.62 \mathrm{E}-03$ & 0.67 & 1.95 & 3.28 \\
\hline 12 & Closed V3 (residual particles) & $3.36 \mathrm{E}-06$ & $5.17 \mathrm{E}-05$ & 0.75 & 2.00 & 3.20 \\
\hline $13-14$ & V3 pressure isolation pulse & $1.93 \mathrm{E}-05$ & $2.97 \mathrm{E}-04$ & 0.79 & 2.15 & 6.56 \\
\hline $15-16$ & V3 pressure isolation pulse & $7.76 \mathrm{E}-06$ & $1.19 \mathrm{E}-04$ & $<0.6$ & 2.21 & 4.28 \\
\hline 17 & $\begin{array}{l}\text { Closed bottle. Set flow controller to } 0.4 \text { SLM. } \\
\text { Opened the bottled, rapidly increasing system pressure to } 7400 \text { torr ( } 0.8 \text { SLM max) }\end{array}$ & 2.10E-05 & $3.23 \mathrm{E}-04$ & 0.81 & 2.23 & 4.45 \\
\hline $18-19$ & Housing tap & $2.21 \mathrm{E}-03$ & $3.39 \mathrm{E}-02$ & 1.10 & 3.39 & 11.28 \\
\hline $20-21$ & Housing tap & $3.43 \mathrm{E}-04$ & $5.28 \mathrm{E}-03$ & 0.80 & 2.24 & 3.75 \\
\hline Total & Sum of all non-background release events & $2.94 \mathrm{E}-03$ & $4.52 \mathrm{E}-02$ & 1.02 & 3.09 & 10.44 \\
\hline
\end{tabular}




\begin{tabular}{|c|c|c|c|c|c|c|c|}
\hline \multicolumn{7}{|c|}{ HBU 539B3 Test 2} & \\
\hline Events & Description & Detected [mg] & $\begin{array}{c}\text { Release } \\
{[\mathrm{mg}]}\end{array}$ & $\begin{array}{c}\mathrm{d} 10 \\
{[\mu \mathrm{m}]}\end{array}$ & $\begin{array}{c}d 50 \\
{[\mu \mathrm{m}]}\end{array}$ & $\begin{array}{c}\mathrm{d} 90 \\
{[\mu \mathrm{m}]}\end{array}$ & \\
\hline 7 & Flow set point increased from 0 to $10 \mathrm{SCCM}$. Flow held at $6.5 \mathrm{SCCM}$. & $2.65 \mathrm{E}-07$ & 4.07E-06 & $<0.6$ & $<0.6$ & $<0.6$ & \\
\hline 9 & Flow set point increased from 10 to $50 \mathrm{SCCM}$. Increased pressure to obtain flow. & $6.37 \mathrm{E}-05$ & 9.80E-04 & 1.56 & 2.75 & 5.58 & \\
\hline 11 & Flow set point increased from 50 to $100 \mathrm{SCCM}$. Increased pressure to obtain flow. & 3.91E-04 & $6.02 \mathrm{E}-03$ & 1.49 & 2.71 & 5.93 & \\
\hline $12-14$ & Flow set point increased from 100 to 150 SCCM. Increased pressure to obtain flow. & $8.78 \mathrm{E}-04$ & 1.35E-02 & 1.22 & 2.60 & 5.77 & \\
\hline $15-16$ & Flow set point increased from 150 to 200 SCCM. Increased pressure to obtain flow. & 5.44E-04 & 8.37E-03 & 1.23 & 2.51 & 4.92 & \\
\hline 17 & Flow controller exchange: FC1 closed, FC2 opened. & $1.52 \mathrm{E}-03$ & 2.33E-02 & 1.11 & 2.53 & 8.13 & \\
\hline 20 & Flow set point increased from 200 to 300 SCCM. Increased pressure to obtain flow. & 3.78E-04 & $5.82 \mathrm{E}-03$ & 1.01 & 2.38 & 4.86 & \\
\hline $21-22$ & Flow set point increased from 300 to $400 \mathrm{SCCM}$. Increased pressure to obtain flow. & $1.52 \mathrm{E}-03$ & 2.34E-02 & 1.04 & 2.43 & 5.27 & \\
\hline 23 & V3 closed (residual particles in system) & $3.24 \mathrm{E}-06$ & 4.98E-05 & 1.18 & 2.30 & 3.26 & \\
\hline $24-25$ & V3 pressure isolation pulse & 9.28E-05 & 1.43E-03 & 1.30 & 2.77 & 12.78 & \\
\hline 26 & V3 pressure isolation pulse & 4.24E-05 & $6.52 \mathrm{E}-04$ & 1.32 & 2.77 & 6.42 & \\
\hline $27-28$ & First housing tap & $5.49 \mathrm{E}-03$ & $8.45 \mathrm{E}-02$ & 1.43 & 3.45 & 15.50 & \\
\hline $29-30$ & Second housing tap & $3.48 \mathrm{E}-03$ & 5.35E-02 & 1.22 & 2.85 & 13.59 & \\
\hline 31 & Closed gas cylinder (residual particles in system) & $2.32 \mathrm{E}-04$ & 3.57E-03 & 4.35 & 9.19 & 14.53 & \\
\hline Total & Sum of all non-background release events & 1.46E-02 & 2.25E-01 & 1.24 & 2.89 & 13.40 & \\
\hline \multicolumn{8}{|c|}{ HBU 539B3 Test 3} \\
\hline Events & \multicolumn{2}{|l|}{ Description } & Detected [mg] & $\begin{array}{c}\text { Release } \\
{[\mathrm{mg}]}\end{array}$ & $\begin{array}{c}\mathrm{d} 10 \\
{[\mu \mathrm{m}]}\end{array}$ & $\begin{array}{c}\mathrm{d} 50 \\
{[\mu \mathrm{m}]}\end{array}$ & $\begin{array}{c}\mathrm{d} 90 \\
{[\mu \mathrm{m}]}\end{array}$ \\
\hline 3 & \multicolumn{2}{|l|}{ Start Experiment. Flow set point increased from 0 to 10 SCCM } & 2.30E-07 & $3.54 \mathrm{E}-06$ & $<0.6$ & $<0.6$ & $<0.6$ \\
\hline 5 & \multicolumn{2}{|l|}{ Increased flow set point from 10 to 50 SCCM. Increased pressure to obtain flow. } & 2.94E-06 & 4.52E-05 & 6.03 & 6.90 & 7.78 \\
\hline 7 & \multicolumn{2}{|l|}{ Increased flow set point from 50 to 100 SCCM. Increased pressure to obtain flow. } & 2.36E-06 & 3.63E-05 & 1.13 & 2.33 & 3.27 \\
\hline 9 & \multicolumn{2}{|l|}{ Increased flow set point from 100 to 150 SCCM. Increased pressure to obtain flow. } & 8.32E-06 & $1.28 \mathrm{E}-04$ & 1.87 & 4.16 & 5.63 \\
\hline 11 & \multicolumn{2}{|l|}{ Increased flow set point from 150 to 200 SCCM. Increased pressure to obtain flow. } & 2.80E-06 & 4.31E-05 & 0.89 & 2.90 & 5.38 \\
\hline 14 & \multicolumn{2}{|l|}{ Increased flow set point from 200 to 300 SCCM. Increased pressure to obtain flow. } & 7.38E-06 & 1.14E-04 & 0.87 & 2.13 & 3.23 \\
\hline $15-16$ & \multicolumn{2}{|c|}{ Increased flow set point from 300 to 400 SCCM. Reached max allowable pressure at 360 SCCM. } & 4.09E-03 & $6.29 \mathrm{E}-02$ & 3.31 & 9.84 & 17.70 \\
\hline 18 & \multicolumn{2}{|c|}{ V3 pressure isolation pulse } & 7.29E-06 & $1.12 \mathrm{E}-04$ & 1.11 & 2.48 & 4.39 \\
\hline 20 & \multicolumn{2}{|l|}{ V3 pressure isolation pulse } & 7.67E-07 & $1.18 \mathrm{E}-05$ & 0.62 & 1.79 & 3.16 \\
\hline 21 & \multicolumn{2}{|l|}{ Accidently tapped either the effluent line or sample holder housing. } & 8.68E-07 & 1.34E-05 & $<0.6$ & 1.57 & 3.11 \\
\hline $24-25$ & First housing tap & & 9.03E-03 & 1.39E-01 & 1.89 & 8.28 & 16.22 \\
\hline $26-27$ & Second housing tap & & $6.98 \mathrm{E}-04$ & 1.07E-02 & 1.12 & 4.29 & 16.29 \\
\hline Total & Sum of all non-background release events & & 1.39E-02 & 2.13E-01 & 2.03 & 8.58 & 16.75 \\
\hline
\end{tabular}




\begin{tabular}{|c|c|c|c|c|c|c|}
\hline \multicolumn{7}{|c|}{ HBU 539D5 } \\
\hline Events & Description & Detected [mg] & Release [mg] & $\mathrm{d} 10[\mu \mathrm{m}]$ & $\mathrm{d} 50[\mu \mathrm{m}]$ & $\mathrm{d} 90[\mu \mathrm{m}]$ \\
\hline $1-2$ & Start experiment; flow controller set to $10 \mathrm{SCCM}$; pressure increased to obtain flow. & 2.43E-05 & 3.74E-04 & 1.56 & 3.03 & 8.69 \\
\hline 3 & Flow set point increased from 10 to 50 SCCM; pressure increased to obtain flow & $6.08 \mathrm{E}-04$ & 9.35E-03 & 1.15 & 2.48 & 5.82 \\
\hline 4 & Flow set point increased from 50 to $100 \mathrm{SCCM}$; pressure increased to obtain flow & $3.68 \mathrm{E}-03$ & $5.66 \mathrm{E}-02$ & 1.13 & 2.37 & 3.46 \\
\hline 5 & Flow set point increased from 100 to $150 \mathrm{SCCM}$; pressure increased to obtain flow & 4.17E-04 & $6.42 \mathrm{E}-03$ & 0.88 & 2.07 & 3.26 \\
\hline 6 & Flow set point increased from 150 to $200 \mathrm{SCCM}$; pressure increased to obtain flow & $5.53 \mathrm{E}-04$ & $8.50 \mathrm{E}-03$ & 0.90 & 2.14 & 3.36 \\
\hline $7-9$ & Flow controller exchange; FC1 closed, FC2 opened, flow set point to 0.2 SLM & 4.51E-06 & 6.94E-05 & 0.85 & 1.95 & 3.19 \\
\hline 10 & Flow set point increased from 200 to $300 \mathrm{SCCM}$; pressure increased to obtain flow & 1.96E-03 & 3.02E-02 & 0.90 & 2.14 & 3.38 \\
\hline 11 & Flow set point increased from 300 to 400 SCCM; pressure increased to obtain flow & 1.57E-03 & $2.41 \mathrm{E}-02$ & 0.87 & 2.10 & 3.44 \\
\hline 12 & $\begin{array}{l}\text { Flow set point increased from } 400 \text { to } 500 \text { SCCM; pressure increased to maximum allowable; } 470 \\
\text { SCCM flow max }\end{array}$ & 7.86E-04 & $1.21 \mathrm{E}-02$ & 0.86 & 2.09 & 3.41 \\
\hline 13 & V3 closed (residual particles through OPC) & $6.55 \mathrm{E}-06$ & $1.01 \mathrm{E}-04$ & 0.95 & 2.17 & 3.23 \\
\hline $14-15$ & V3 pressure isolation pulse & $2.65 \mathrm{E}-04$ & $4.08 \mathrm{E}-03$ & 0.89 & 2.18 & 3.53 \\
\hline 16 & V3 pressure isolation pulse & $9.41 \mathrm{E}-05$ & 1.45E-03 & 0.89 & 2.09 & 3.39 \\
\hline 18 & Gas bottle pressure isolation pulse (closed, then opened bottle) & 1.10E-05 & 1.70E-04 & 0.86 & 2.06 & 3.21 \\
\hline 19 & Housing tap & 4.76E-03 & 7.32E-02 & 0.94 & 2.41 & 9.41 \\
\hline 20 & Gas bottle pressure isolation pulse (closed, then opened bottle) & 8.47E-07 & $1.30 \mathrm{E}-05$ & 0.66 & 2.24 & 3.25 \\
\hline Total & Sum of all non-background release events & $1.47 \mathrm{E}-02$ & 2.27E-01 & 0.96 & 2.29 & 4.62 \\
\hline
\end{tabular}




\begin{tabular}{|c|c|c|c|c|c|c|}
\hline \multicolumn{7}{|c|}{ "HBU 539F5 Test 1} \\
\hline Events & Description & Detected [mg] & $\begin{array}{l}\text { Release } \\
\text { [mg] }\end{array}$ & $\begin{array}{c}\mathrm{d} 10 \\
{[\mu \mathrm{m}]}\end{array}$ & $\begin{array}{c}\mathrm{d} 50 \\
{[\mu \mathrm{m}]}\end{array}$ & $\begin{array}{c}\mathrm{d} 90 \\
{[\mu \mathrm{m}]}\end{array}$ \\
\hline 3 & Flow set point increased from 50 to $100 \mathrm{SCCM}$. & $1.20 \mathrm{E}-05$ & $1.84 \mathrm{E}-04$ & 1.50 & 2.39 & 3.28 \\
\hline 4 & Flow set point increased from 100 to $150 \mathrm{SCCM}$. & $4.36 \mathrm{E}-05$ & $6.70 \mathrm{E}-04$ & 1.57 & 2.43 & 3.29 \\
\hline 5 & Flow set point increased from 150 to $200 \mathrm{SCCM}$. & $1.43 \mathrm{E}-04$ & $2.19 \mathrm{E}-03$ & 1.60 & 2.48 & 3.35 \\
\hline 6 & Flow controller exchange: FC1 close, FC2 open & $4.24 \mathrm{E}-03$ & $6.52 \mathrm{E}-02$ & 1.70 & 3.02 & 12.93 \\
\hline $10-11$ & First housing tap & $6.29 \mathrm{E}-03$ & $9.67 \mathrm{E}-02$ & 1.53 & 2.58 & 5.88 \\
\hline $12-13$ & Second housing tap & $4.73 \mathrm{E}-03$ & $7.27 \mathrm{E}-02$ & 1.41 & 2.49 & 3.81 \\
\hline $14-15$ & Air inlet line to sample chamber tapped. & $5.66 \mathrm{E}-03$ & 8.71E-02 & 1.40 & 2.54 & 5.01 \\
\hline 19 & Flow set point increased from 800 to 900 SCCM. & $2.50 \mathrm{E}-06$ & $3.84 \mathrm{E}-05$ & 1.49 & 2.39 & 3.28 \\
\hline 20 & Flow set point increased from 900 to 1000 SCCM. & $5.47 \mathrm{E}-07$ & $8.42 \mathrm{E}-06$ & 0.97 & 2.28 & 3.26 \\
\hline 21 & Flow set point increased from 1.0 to $1.5 \mathrm{SLM}$. & $3.67 \mathrm{E}-04$ & $5.65 \mathrm{E}-03$ & 1.13 & 2.38 & 3.51 \\
\hline 22 & Flow set point increased from 1.5 to 2.0 SLM. & $2.67 \mathrm{E}-03$ & $4.10 \mathrm{E}-02$ & 1.23 & 2.41 & 3.47 \\
\hline 23 & Flow set point increased from 2.0 to $2.5 \mathrm{SLM}$. & $2.35 \mathrm{E}-06$ & $3.62 \mathrm{E}-05$ & 1.28 & 2.34 & 3.27 \\
\hline 24 & Flow set point increased from 2.5 to $3.0 \mathrm{SLM}$. & $4.93 \mathrm{E}-04$ & $7.58 \mathrm{E}-03$ & 1.15 & 2.48 & 5.53 \\
\hline 25 & Flow set point decreased from 3.0 to 0.2 SLM. & $1.48 \mathrm{E}-06$ & $2.28 \mathrm{E}-05$ & 1.05 & 2.18 & 3.24 \\
\hline 26 & Flow set point decreased from 0.2 to 3.0 SLM. & $1.88 \mathrm{E}-05$ & $2.89 \mathrm{E}-04$ & 1.13 & 2.33 & 3.39 \\
\hline $27-28$ & Third housing tap & $1.38 \mathrm{E}-03$ & $2.12 \mathrm{E}-02$ & 1.28 & 2.78 & 11.09 \\
\hline $29-30$ & Fourth housing tap & 8.97E-04 & $1.38 \mathrm{E}-02$ & 1.14 & 2.36 & 3.43 \\
\hline $31-32$ & Second air inlet tap & $6.14 \mathrm{E}-03$ & $9.44 \mathrm{E}-02$ & 1.29 & 2.68 & 11.87 \\
\hline $33-34$ & Third air inlet tap & $4.41 \mathrm{E}-03$ & 6.79E-02 & 2.05 & 5.46 & 16.26 \\
\hline 36 & V3 pressure isolation pulse & $5.91 \mathrm{E}-04$ & $9.10 \mathrm{E}-03$ & 1.83 & 5.66 & 15.89 \\
\hline $38-39$ & V3 pressure isolation pulse & $3.59 \mathrm{E}-04$ & $5.53 \mathrm{E}-03$ & 1.80 & 7.68 & 16.07 \\
\hline 40 & V3 pressure isolation pulse & $2.38 \mathrm{E}-03$ & $3.65 \mathrm{E}-02$ & 1.67 & 3.43 & 10.47 \\
\hline 42 & V3 pressure isolation pulse & $1.02 \mathrm{E}-03$ & $1.56 \mathrm{E}-02$ & 1.68 & 4.47 & 15.38 \\
\hline 44 & V3 pressure isolation pulse & $4.58 \mathrm{E}-04$ & 7.05E-03 & 1.69 & 4.48 & 15.21 \\
\hline 46 & V3 pressure isolation pulse & $1.88 \mathrm{E}-03$ & $2.90 \mathrm{E}-02$ & 1.16 & 2.41 & 3.85 \\
\hline 48 & V3 pressure isolation pulse & $4.32 \mathrm{E}-04$ & $6.64 \mathrm{E}-03$ & 1.38 & 3.02 & 14.60 \\
\hline 50 & V3 pressure isolation pulse & $1.23 \mathrm{E}-03$ & $1.89 \mathrm{E}-02$ & 1.11 & 2.50 & 9.29 \\
\hline 52 & V3 pressure isolation pulse & $6.18 \mathrm{E}-04$ & $9.50 \mathrm{E}-03$ & $1.4+$ & 3.21 & 16.25 \\
\hline 55 & V3 pressure isolation pulse & $8.57 \mathrm{E}-04$ & $1.32 \mathrm{E}-02$ & 1.23 & 2.46 & 4.33 \\
\hline 56 & Flow set point decreased from 9.0 to 3.0 SLM (residual particles) & $5.14 \mathrm{E}-07$ & 7.91E-06 & 1.33 & 2.36 & 3.27 \\
\hline $57-58$ & Fifth housing tap & $3.95 \mathrm{E}-03$ & $6.07 \mathrm{E}-02$ & 3.30 & 9.28 & 15.29 \\
\hline $59-60$ & Fourth air inlet tap & $6.37 \mathrm{E}-04$ & $9.80 \mathrm{E}-03$ & 2.09 & 9.46 & 15.84 \\
\hline Total & Sum of all non-background release events & $5.19 \mathrm{E}-02$ & $7.98 \mathrm{E}-01$ & 1.51 & 2.85 & 11.59 \\
\hline
\end{tabular}




\begin{tabular}{|c|c|c|c|c|c|c|}
\hline \multicolumn{7}{|c|}{ HBU 539F5 Test 2} \\
\hline Events & Description & Detected [mg] & $\begin{array}{c}\text { Release } \\
{[\mathrm{mg}]}\end{array}$ & $\begin{array}{c}\mathrm{d} 10 \\
{[\mu \mathrm{m}]}\end{array}$ & $\begin{array}{c}\mathrm{d} 50 \\
{[\mu \mathrm{m}]}\end{array}$ & $\begin{array}{c}\mathrm{d} 90 \\
{[\mu \mathrm{m}]}\end{array}$ \\
\hline 3 & Increased flow set point from 10 to $50 \mathrm{SCCM}$ & $1.13 \mathrm{E}-03$ & 1.75E-02 & 1.20 & 2.45 & 4.17 \\
\hline $4-5$ & Increased flow set point from 50 to $100 \mathrm{SCCM}$; increased pressure to obtain flow & $1.42 \mathrm{E}-03$ & 2.19E-02 & 1.09 & 2.39 & 4.17 \\
\hline $6-7$ & Increased flow set point from 100 to $150 \mathrm{SCCM}$; increased pressure to obtain flow & $1.25 \mathrm{E}-03$ & 1.93E-02 & 1.03 & 2.30 & 3.44 \\
\hline 8 & Increased flow set point from 150 to $200 \mathrm{SCCM}$ & $1.06 \mathrm{E}-03$ & 1.63E-02 & 1.03 & 2.38 & 5.38 \\
\hline 9 & Flow controller exchange: FC1 closed, FC2 opened. & 3.44E-05 & 5.30E-04 & 0.94 & 2.12 & 3.22 \\
\hline $10-11$ & Increased flow set point from 200 to 300 SCCM; increased pressure to obtain flow & $2.02 E-03$ & $3.11 \mathrm{E}-02$ & 0.98 & 2.30 & 4.12 \\
\hline $12-14$ & Increased flow set point from 300 to $400 \mathrm{SCCM}$; increased pressure to obtain flow & 2.17E-03 & 3.34E-02 & 0.95 & 2.27 & 4.21 \\
\hline $15-16$ & Increased flow set point from 400 to $500 \mathrm{SCCM}$; increased pressure to obtain flow & $1.88 \mathrm{E}-03$ & $2.90 \mathrm{E}-02$ & 0.96 & 2.32 & 5.20 \\
\hline $17-18$ & Increased flow set point from 500 to $750 \mathrm{SCCM}$; increased pressure to obtain flow & $3.91 \mathrm{E}-03$ & 6.01E-02 & 0.96 & 2.33 & 5.51 \\
\hline 19 & Decreased flow set point from 750 to $500 \mathrm{SCCM}$ & $1.12 \mathrm{E}-04$ & $1.72 \mathrm{E}-03$ & 1.00 & 2.47 & 5.74 \\
\hline $20-21$ & First housing tap & $8.56 \mathrm{E}-03$ & 1.32E-01 & 1.01 & 2.36 & 5.35 \\
\hline $22-23$ & Air inlet tap & $1.50 \mathrm{E}-03$ & $2.31 \mathrm{E}-02$ & 1.00 & 2.34 & 4.37 \\
\hline 24 & V3 closed (residual particles in line) & $6.92 \mathrm{E}-06$ & 1.06E-04 & 1.07 & 2.27 & 3.25 \\
\hline 25 & V3 pressure isolation pulse & $1.01 \mathrm{E}-04$ & 1.56E-03 & 1.11 & 2.77 & 14.98 \\
\hline 26 & Decreased flow set point from 500 to 0 SCCM (residual particles in line) & 7.43E-06 & 1.14E-04 & 0.86 & 2.29 & 4.36 \\
\hline $27-28$ & Second housing tap (no flow) & $8.43 E-05$ & 1.30E-03 & 1.19 & 2.46 & 4.64 \\
\hline 29 & Line connecting sample chamber to OPC tapped. & 3.47E-04 & 5.34E-03 & 7.51 & 17.46 & 18.69 \\
\hline Total & Sum of all non-background release events & $2.56 \mathrm{E}-02$ & 3.94E-01 & 1.01 & 2.36 & 5.18 \\
\hline
\end{tabular}




\section{Appendix B}

\section{Mass Release from CSNF Powder Tests}

This appendix is composed of tables containing the particle mass released and the particle mass detected for each CSNF powder test. The column labeled "Detected" represents the mass of particles determined from OPC measurements. The column labeled "Release" represents the mass difference measured in the sample. Particle-size distributions generated from the data collected by the OPC are also presented. 


\section{Appendix B: Mass Release from CSNF Powder Tests}

\begin{tabular}{|c|c|c|c|c|c|c|}
\hline \multicolumn{7}{|c|}{ 105-HIGH } \\
\hline Events & Description & Detected $[\mathrm{mg}]$ & $\begin{array}{c}\text { Release } \\
{[\mathrm{mg}]}\end{array}$ & $\begin{array}{c}\mathrm{d} 10 \\
{[\mu \mathrm{m}]}\end{array}$ & $\begin{array}{c}\mathrm{d} 50 \\
{[\mu \mathrm{m}]}\end{array}$ & $\begin{array}{c}\mathrm{d} 90 \\
{[\mu \mathrm{m}]}\end{array}$ \\
\hline 12 & Flow Controller Switch-Over: FC1 to FC2 & $5.67 \mathrm{E}-04$ & $8.72 \mathrm{E}-03$ & 2.62 & 9.01 & 16.70 \\
\hline 22 & Increased flow from 1.25 to $1.50 \mathrm{SLM}$ & $4.30 \mathrm{E}-05$ & $6.61 \mathrm{E}-04$ & 2.30 & 8.24 & 10.22 \\
\hline 23 & Increased flow from 1.50 to $1.75 \mathrm{SLM}$ & $1.45 \mathrm{E}-04$ & $2.23 \mathrm{E}-03$ & 3.96 & 13.57 & 18.27 \\
\hline 24 & Increased flow from 1.75 to $2.00 \mathrm{SLM}$ & $4.11 \mathrm{E}-05$ & $6.32 \mathrm{E}-04$ & 2.99 & 9.07 & 14.19 \\
\hline 28 & Increased flow from 2.75 to $3.00 \mathrm{SLM}$ & $3.37 \mathrm{E}-05$ & $5.18 \mathrm{E}-04$ & 4.50 & 12.09 & 14.25 \\
\hline 29 & Accidental bump of housing lid with manipulator & $1.53 \mathrm{E}-04$ & $2.35 \mathrm{E}-03$ & 2.20 & 6.48 & 11.89 \\
\hline 30 & Gentle housing tap & $3.49 \mathrm{E}-05$ & $5.36 \mathrm{E}-04$ & 3.13 & 10.06 & 13.18 \\
\hline 32 & Rough housing tap & $1.74 \mathrm{E}-03$ & $2.67 \mathrm{E}-02$ & 2.96 & 9.84 & 17.33 \\
\hline $34-35$ & Lid tap & $9.31 \mathrm{E}-04$ & $1.43 \mathrm{E}-02$ & 3.40 & 10.62 & 17.80 \\
\hline 39 & Increased flow from 4 to 5 SLM & $7.86 \mathrm{E}-05$ & $1.21 \mathrm{E}-03$ & 3.56 & 10.14 & 15.23 \\
\hline 40 & Increased flow from 5 to 6 SLM & $7.37 \mathrm{E}-05$ & $1.13 \mathrm{E}-03$ & 4.23 & 9.67 & 10.31 \\
\hline 44 & Increase flow from 8 to 9 SLM & $1.47 \mathrm{E}-05$ & $2.27 \mathrm{E}-04$ & 13.50 & 13.95 & 14.39 \\
\hline 45 & Inlet tap & 3.59E-05 & $5.52 \mathrm{E}-04$ & 5.75 & 14.40 & 15.28 \\
\hline $47-48$ & Rough housing tap & $4.27 \mathrm{E}-04$ & $6.57 \mathrm{E}-03$ & 3.42 & 10.09 & 18.35 \\
\hline $53-54$ & $\mathrm{~V} 3$ pressure isolation pulse & 4.40E-05 & $6.77 \mathrm{E}-04$ & 1.70 & 3.42 & 9.63 \\
\hline $55-56$ & Rough housing tap with no gas flow & $3.11 \mathrm{E}-05$ & $4.79 \mathrm{E}-04$ & 2.26 & 6.71 & 9.08 \\
\hline 57 & V3 pressure isolation pulse & $1.13 E-04$ & $1.74 \mathrm{E}-03$ & 2.00 & 6.41 & 10.22 \\
\hline 58 & Rough housing tap & $2.54 \mathrm{E}-04$ & $3.91 \mathrm{E}-03$ & 2.64 & 12.35 & 15.15 \\
\hline Total & Sum of all non-background release events & $4.76 \mathrm{E}-03$ & $7.32 \mathrm{E}-02$ & 2.92 & 9.97 & 17.46 \\
\hline
\end{tabular}




\begin{tabular}{|c|c|c|c|c|c|c|}
\hline \multicolumn{7}{|c|}{ 105-LOW } \\
\hline Events & Description & Detected [mg] & $\begin{array}{c}\text { Release } \\
{[\mathrm{mg}]}\end{array}$ & $\begin{array}{c}d 10 \\
{[\mu \mathrm{m}]}\end{array}$ & $\begin{array}{c}\mathrm{d} 50 \\
{[\mu \mathrm{m}]}\end{array}$ & $\begin{array}{c}d 90 \\
{[\mu \mathrm{m}]}\end{array}$ \\
\hline 3 & Start. Open V1, activate $\mathrm{FC} 1$, and open V3. & 4.71E-05 & $7.25 \mathrm{E}-04$ & 2.75 & 9.48 & 14.15 \\
\hline 12 & Flow controller switch: FC1 off, FC2 on. & $7.78 \mathrm{E}-03$ & $1.20 \mathrm{E}-01$ & 1.79 & 5.27 & 14.42 \\
\hline 25 & Increase flow from 2.00 SLM to 2.25 SLM & $6.69 \mathrm{E}-06$ & $1.03 E-04$ & 1.72 & 8.16 & 9.23 \\
\hline 26 & Increase flow from $2.25 \mathrm{SLM}$ to $2.50 \mathrm{SLM}$ & $1.70 \mathrm{E}-03$ & $2.61 \mathrm{E}-02$ & 1.87 & 6.25 & 15.85 \\
\hline $31-32$ & Rough housing tap. & $1.81 \mathrm{E}-04$ & $2.78 \mathrm{E}-03$ & 2.09 & 8.25 & 13.38 \\
\hline 33-34 & Lid tap. & 1.30E-04 & 2.00E-03 & 2.18 & 8.98 & 15.10 \\
\hline $35-36$ & Shake of line from sample holder to OPC. & 5.09E-04 & 7.83E-03 & 2.48 & 10.12 & 16.68 \\
\hline 40 & Increase flow from 6 SLM to 7 SLM & $2.36 \mathrm{E}-05$ & 3.63E-04 & 8.35 & 13.61 & 14.32 \\
\hline 42 & Increase flow from 8 SLM to 9 SLM & $2.13 E-05$ & $3.27 \mathrm{E}-04$ & 4.13 & 11.26 & 12.25 \\
\hline 45 & Housing tap. & $2.52 \mathrm{E}-05$ & 3.87E-04 & 3.09 & 9.70 & 12.21 \\
\hline 51 & V3 pressure isolation pulse & $5.14 \mathrm{E}-04$ & $7.90 \mathrm{E}-03$ & 2.36 & 8.40 & 13.42 \\
\hline 53 & V3 pressure isolation pulse & 2.43E-05 & 3.73E-04 & 1.80 & 13.59 & 14.32 \\
\hline 54 & Housing tap. & 1.45E-05 & $2.23 E-04$ & 3.36 & 9.78 & 10.36 \\
\hline Total & Sum of all non-background release events & 1.10E-02 & 1.69E-01 & 1.84 & 5.94 & 14.99 \\
\hline
\end{tabular}

\begin{tabular}{|c|c|c|c|c|c|c|}
\hline \multicolumn{7}{|c|}{ 109-A450 } \\
\hline Events & Description & Detected [mg] & $\begin{array}{c}\text { Release } \\
\text { [mg] }\end{array}$ & $\begin{array}{c}\mathrm{d} 10 \\
{[\mu \mathrm{m}]}\end{array}$ & $\begin{array}{c}\mathrm{d} 50 \\
{[\mu \mathrm{m}]}\end{array}$ & $\begin{array}{c}d 90 \\
{[\mu \mathrm{m}]}\end{array}$ \\
\hline 11 & Flow controller switch: FC2 on, FC1 off (with flow off) & 2.93E-04 & $4.51 \mathrm{E}-03$ & 3.71 & 12.69 & 16.34 \\
\hline 27 & Increased flow from 3 to 9 SLM & 4.59E-05 & $7.06 \mathrm{E}-04$ & 3.24 & 10.17 & 15.22 \\
\hline 32 & V3 pressure isolation pulse & $9.21 \mathrm{E}-06$ & $1.42 \mathrm{E}-04$ & 11.53 & 11.96 & 12.39 \\
\hline 41 & V3 pressure isolation pulse & $7.21 \mathrm{E}-07$ & $1.11 \mathrm{E}-05$ & 1.12 & 2.22 & 3.24 \\
\hline 44 & V3 pressure isolation pulse & 1.93E-05 & $2.97 \mathrm{E}-04$ & 5.93 & 10.28 & 12.27 \\
\hline Total & Sum of all non-background release events & 3.69E-04 & $5.67 \mathrm{E}-03$ & 3.63 & 12.19 & 16.08 \\
\hline
\end{tabular}




\begin{tabular}{|c|c|c|c|c|c|c|}
\hline \multicolumn{7}{|c|}{ 109-A500 } \\
\hline Events & Description & Detected [mg] & $\begin{array}{c}\text { Release } \\
{[\mathrm{mg}]}\end{array}$ & $\begin{array}{c}\text { d10 } \\
{[\mu \mathrm{m}]}\end{array}$ & $\begin{array}{c}\mathrm{d} 50 \\
{[\mu \mathrm{m}]}\end{array}$ & $\begin{array}{c}\mathrm{d} 90 \\
{[\mu \mathrm{m}]}\end{array}$ \\
\hline 5 & Increased flow from 3 to $5 \mathrm{SCCM}$ & 8.50E-08 & $1.31 \mathrm{E}-06$ & $<0.6$ & $<0.6$ & $<0.6$ \\
\hline 6 & Increased flow from 5 to $10 \mathrm{SCCM}$ & $9.44 \mathrm{E}-09$ & 1.45E-07 & $<0.6$ & $<0.6$ & $<0.6$ \\
\hline 21 & Increased flow from 150 to $160 \mathrm{SCCM}$ & $1.08 \mathrm{E}-06$ & 1.66E-05 & 1.34 & 2.35 & 3.27 \\
\hline 28 & FC exchange: Open V2 & 3.41E-05 & 5.24E-04 & 1.73 & 3.23 & 12.10 \\
\hline 30 & Increased flow from 200 to $500 \mathrm{SCCM}$ & $2.58 \mathrm{E}-06$ & 3.97E-05 & 1.59 & 2.44 & 3.29 \\
\hline 38 & Increased flow from 600 to $700 \mathrm{SCCM}$ & 7.57E-05 & 1.16E-03 & 1.66 & 3.49 & 15.04 \\
\hline 39 & Increased flow from 700 to $800 \mathrm{SCCM}$ & 2.99E-05 & 4.60E-04 & 1.45 & 2.62 & 4.68 \\
\hline 40 & Increased flow from 800 to $900 \mathrm{SCCM}$ & $6.12 \mathrm{E}-06$ & $9.41 \mathrm{E}-05$ & 1.66 & 2.91 & 5.19 \\
\hline 41 & Increased flow from 900 to $1000 \mathrm{SCCM}$ & 8.43E-06 & 1.30E-04 & 1.43 & 2.50 & 3.77 \\
\hline 43 & Increased flow from 1.00 to 1.25 SLM & 2.09E-04 & $3.22 \mathrm{E}-03$ & 1.70 & 6.98 & 16.03 \\
\hline 44 & Increased flow from 1.25 to 1.50 SLM & 9.94E-06 & 1.53E-04 & 1.61 & 3.38 & 9.10 \\
\hline $45-46$ & Increased flow from 1.50 to $1.75 \mathrm{SLM}$, increased pressure & 2.31E-04 & 3.55E-03 & 1.63 & 4.18 & 17.70 \\
\hline 48 & Increased flow from 2.00 to 2.25 SLM & 2.79E-06 & 4.29E-05 & 1.03 & 2.15 & 3.23 \\
\hline $52-53$ & Increased flow from 2.75 to 3.00 SLM, increased pressure & $9.21 \mathrm{E}-05$ & 1.42E-03 & 1.41 & 3.03 & 9.43 \\
\hline 58 & V3 pressure isolation pulse & 7.80E-05 & 1.20E-03 & 1.62 & 8.25 & 14.18 \\
\hline 59 & Increase flow from 3 to 5 SLM & 3.37E-03 & $5.18 \mathrm{E}-02$ & 1.83 & 4.75 & 15.20 \\
\hline 61 & V3 pressure isolation pulse & 1.40E-04 & 2.15E-03 & 2.44 & 8.55 & 13.98 \\
\hline 63 & Increase flow from 7 to 9 SLM & 2.35E-05 & 3.62E-04 & 2.14 & 6.54 & 10.27 \\
\hline 65 & Increased pressure from 5970 to 7390 torr & $6.56 \mathrm{E}-05$ & $1.01 \mathrm{E}-03$ & 2.32 & 10.11 & 14.27 \\
\hline 67 & V3 pressure isolation pulse & 4.35E-04 & $6.70 \mathrm{E}-03$ & 3.74 & 13.13 & 16.77 \\
\hline 73 & Housing tap & 4.46E-05 & $6.86 \mathrm{E}-04$ & 2.59 & 7.57 & 10.49 \\
\hline Total & Sum of all non-background release events & 5.00E-03 & 7.69E-02 & 1.83 & 5.40 & 15.69 \\
\hline
\end{tabular}





\section{Appendix C}

\section{Particle-Size Distributions from CSNF Rod Tests}

This appendix contains a histogram of the particle-size distribution for each significant event of each CSNF rod test. Each size bin in the histogram presents the volume fraction and the number fraction of the total particle population belonging to the bin. 


\section{Appendix C: Particle-Size Distributions from CSNF Rod Tests}
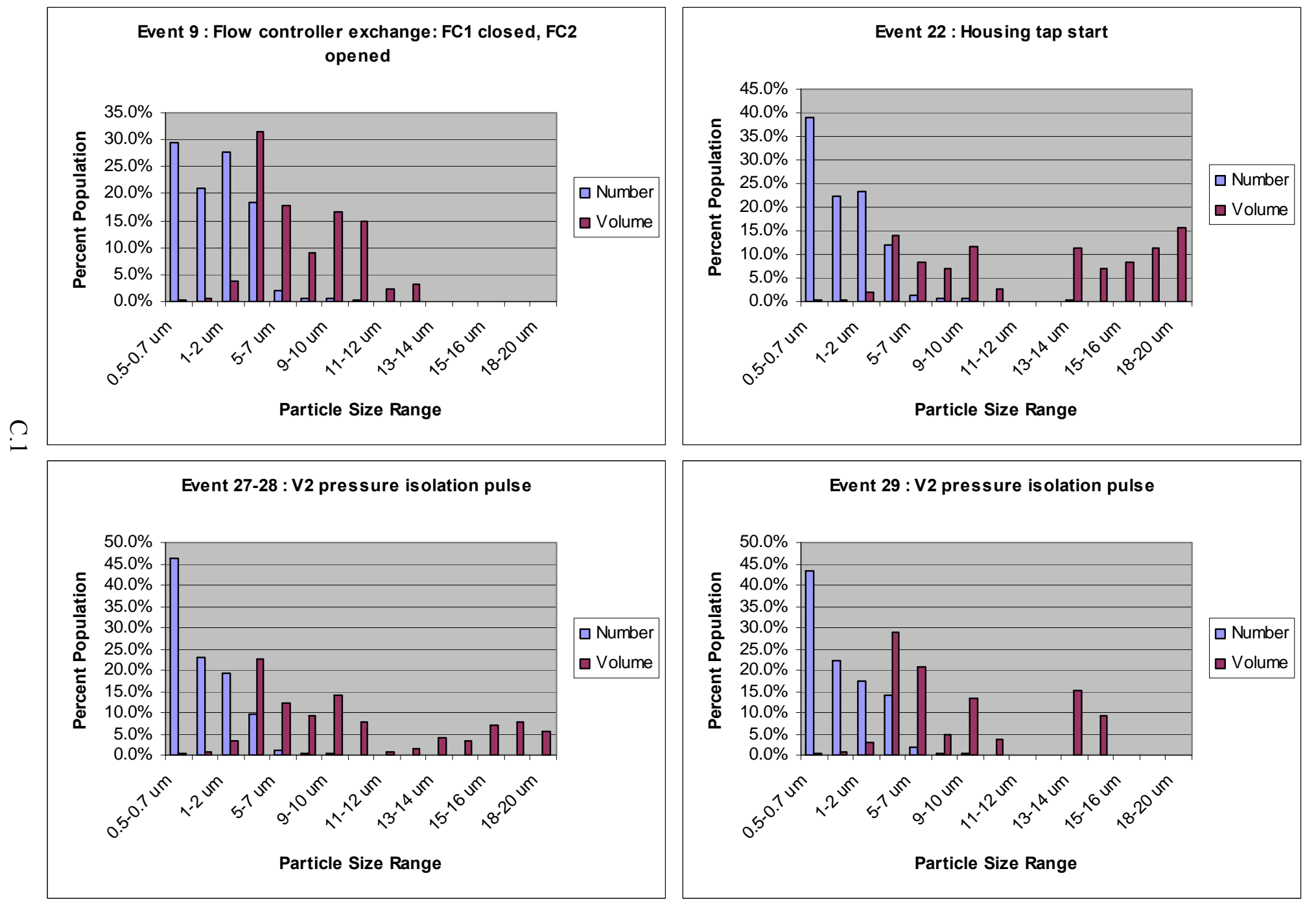

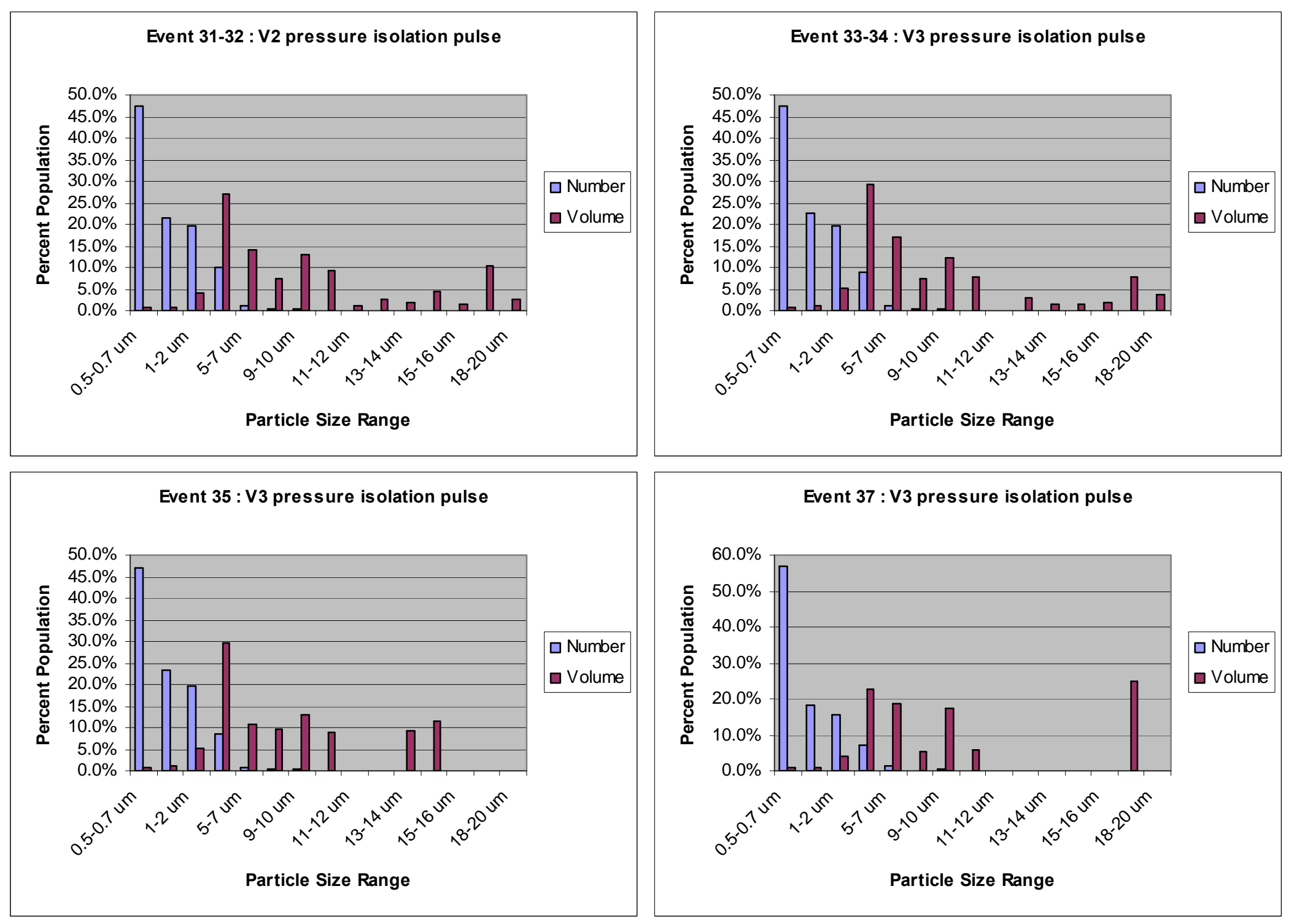

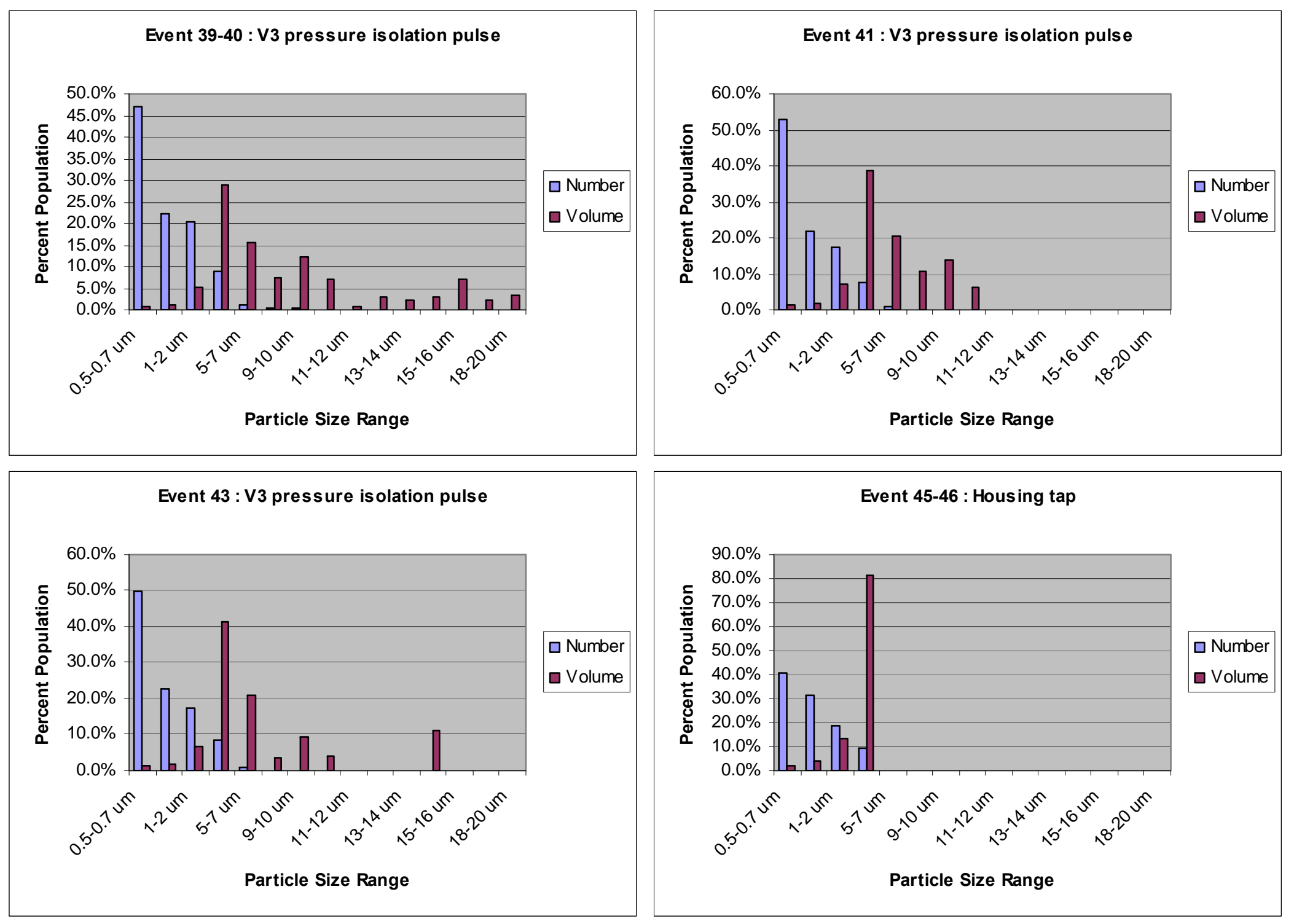

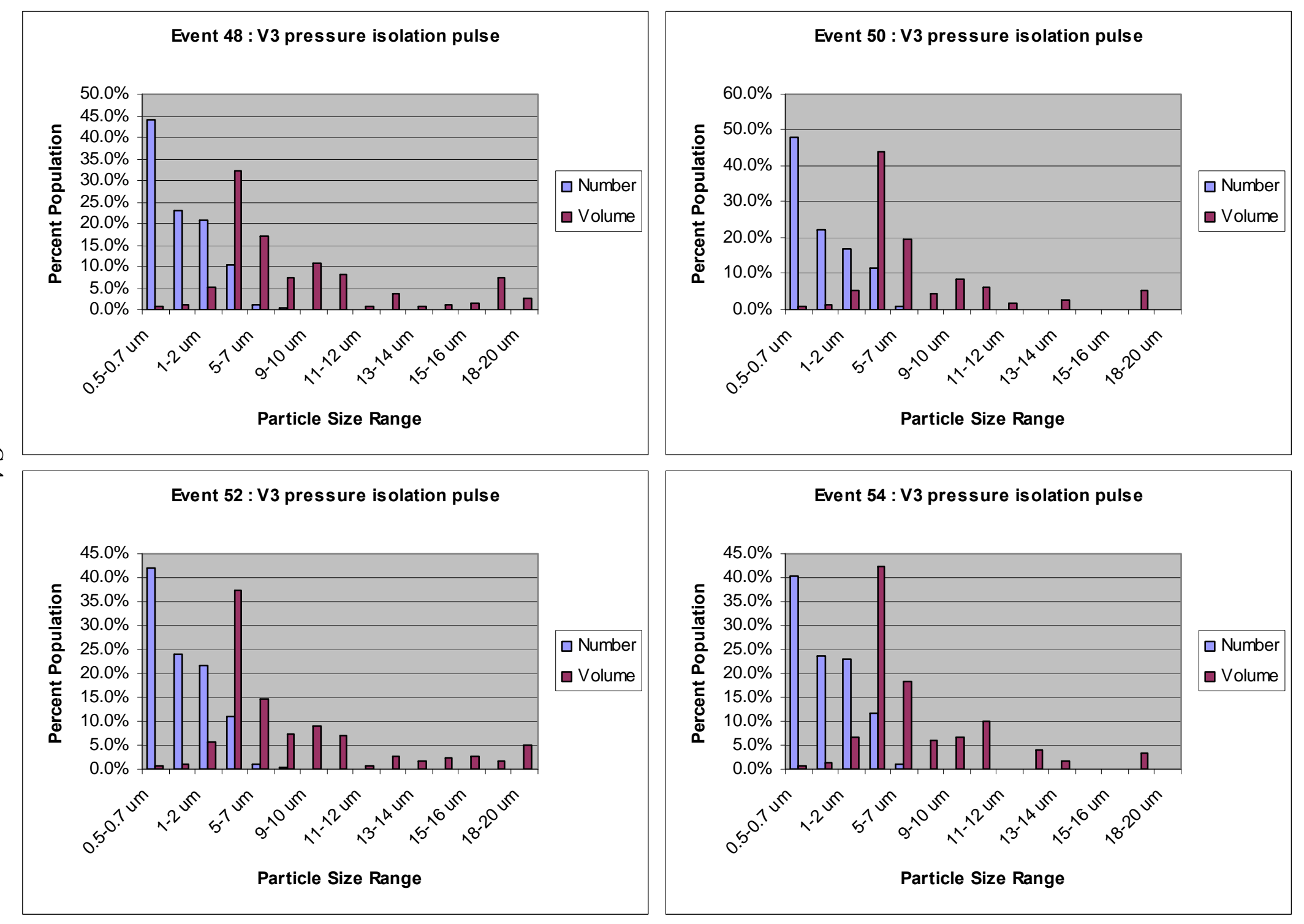

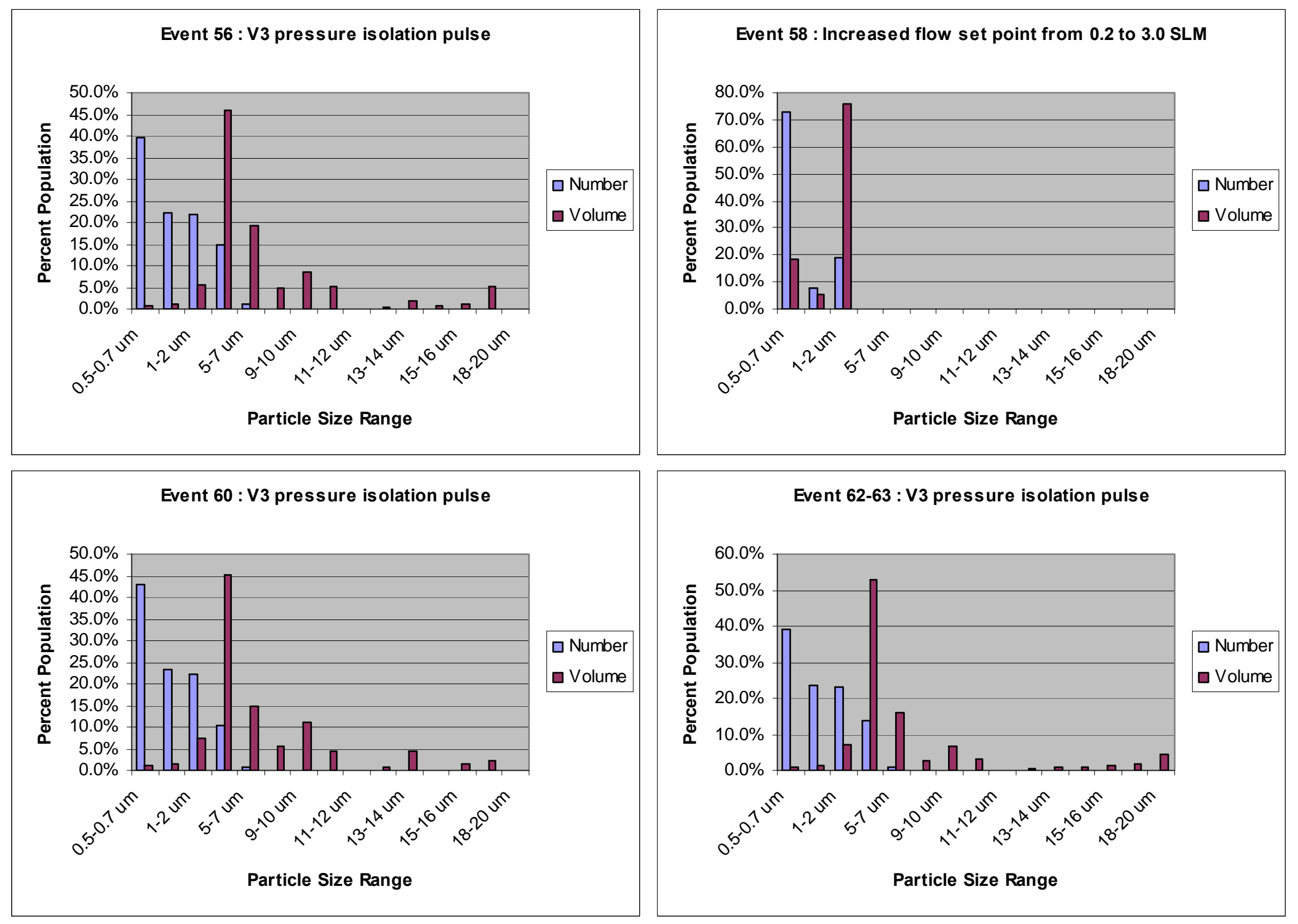

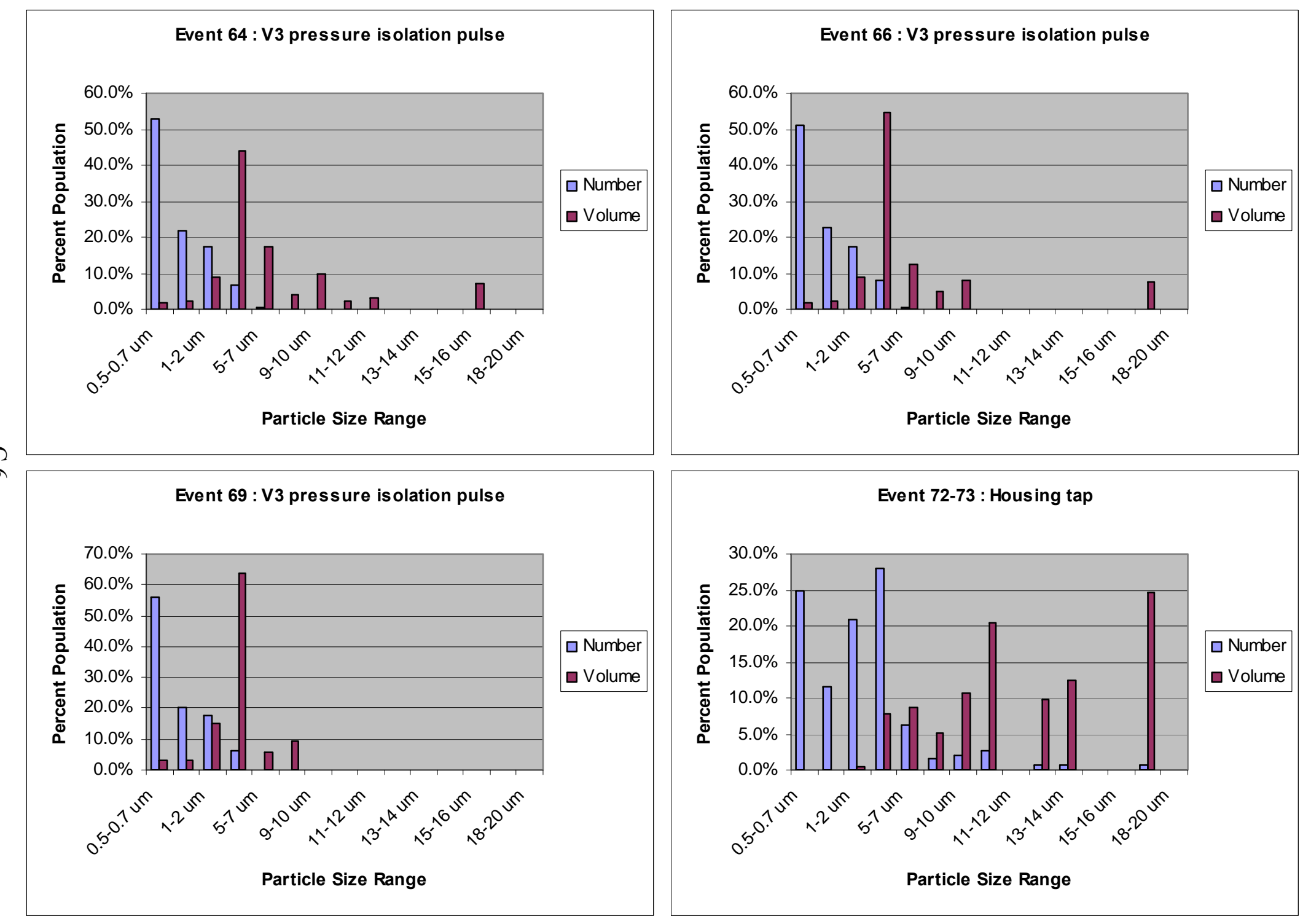
ATM106 Test 1

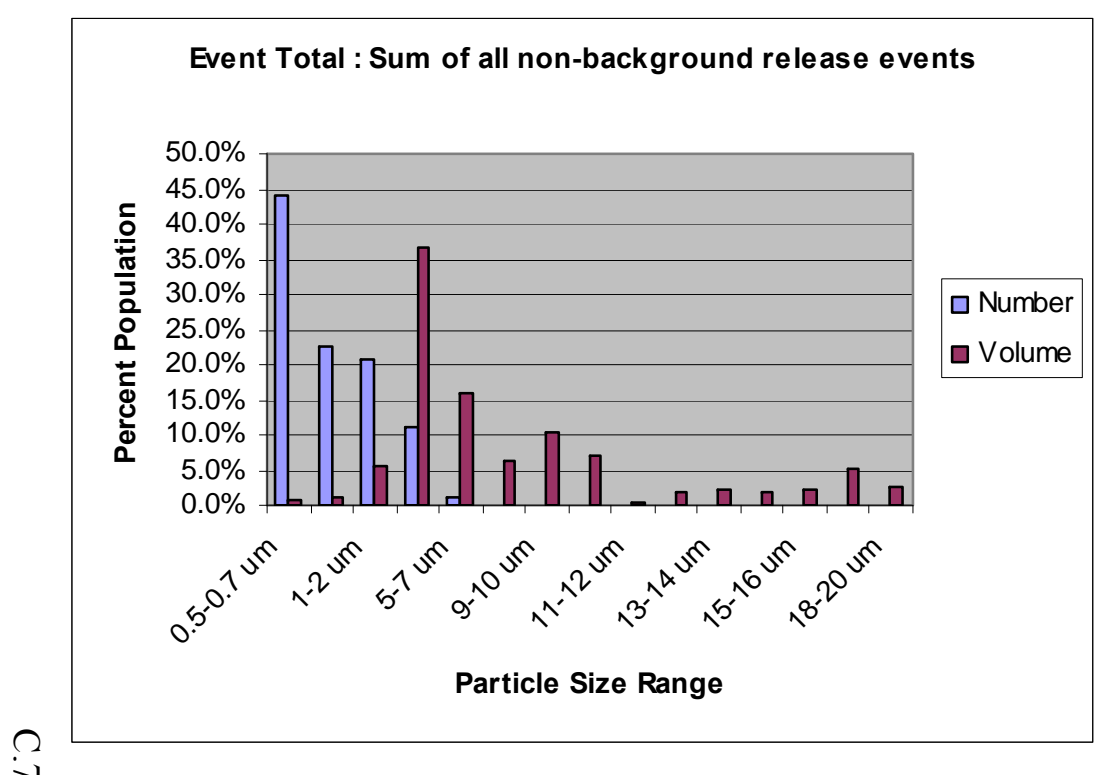



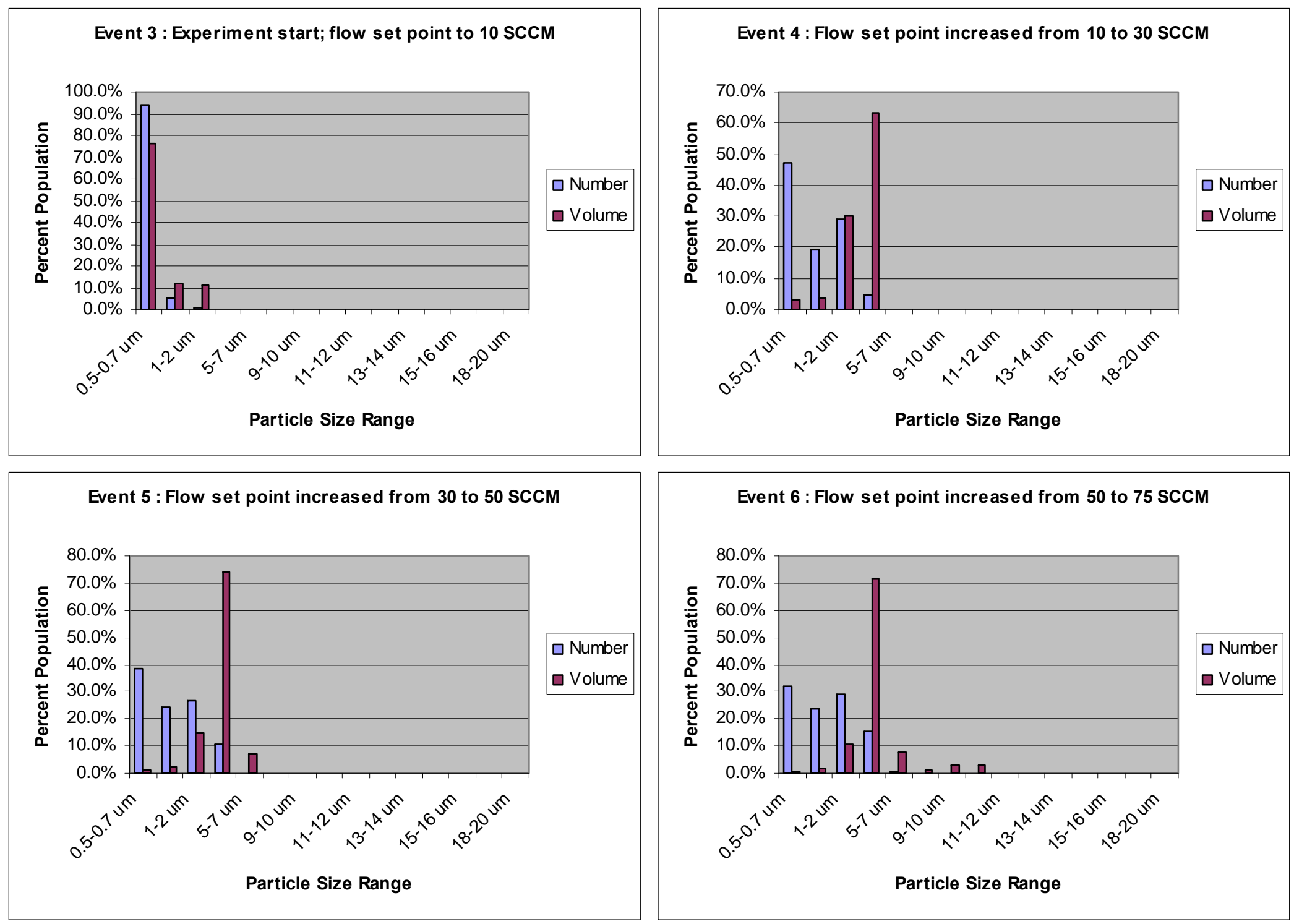

\section{Event 6 : Flow set point increased from 50 to 75 SCCM}

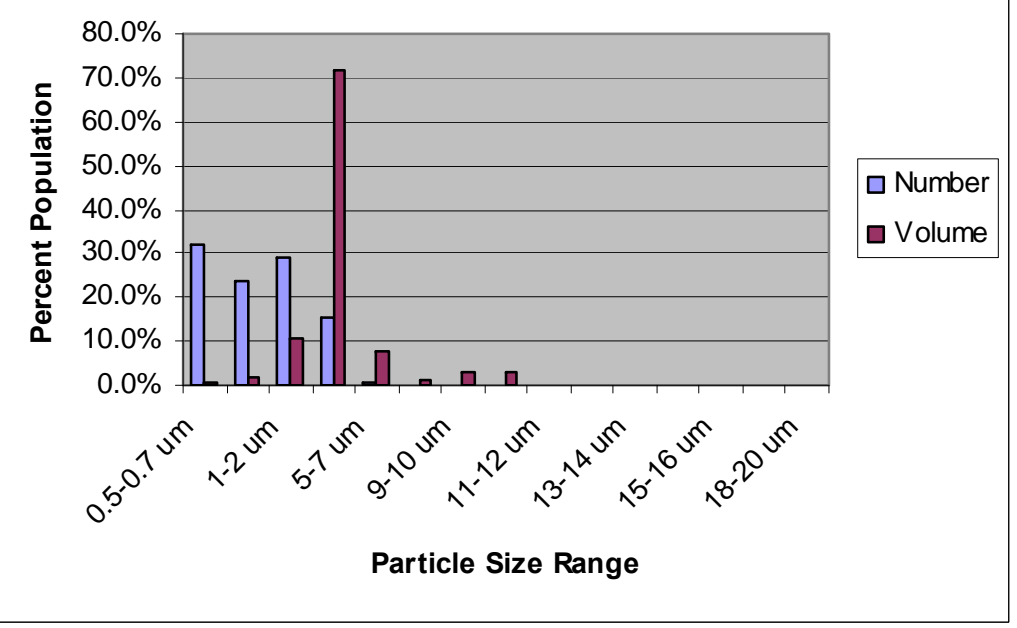



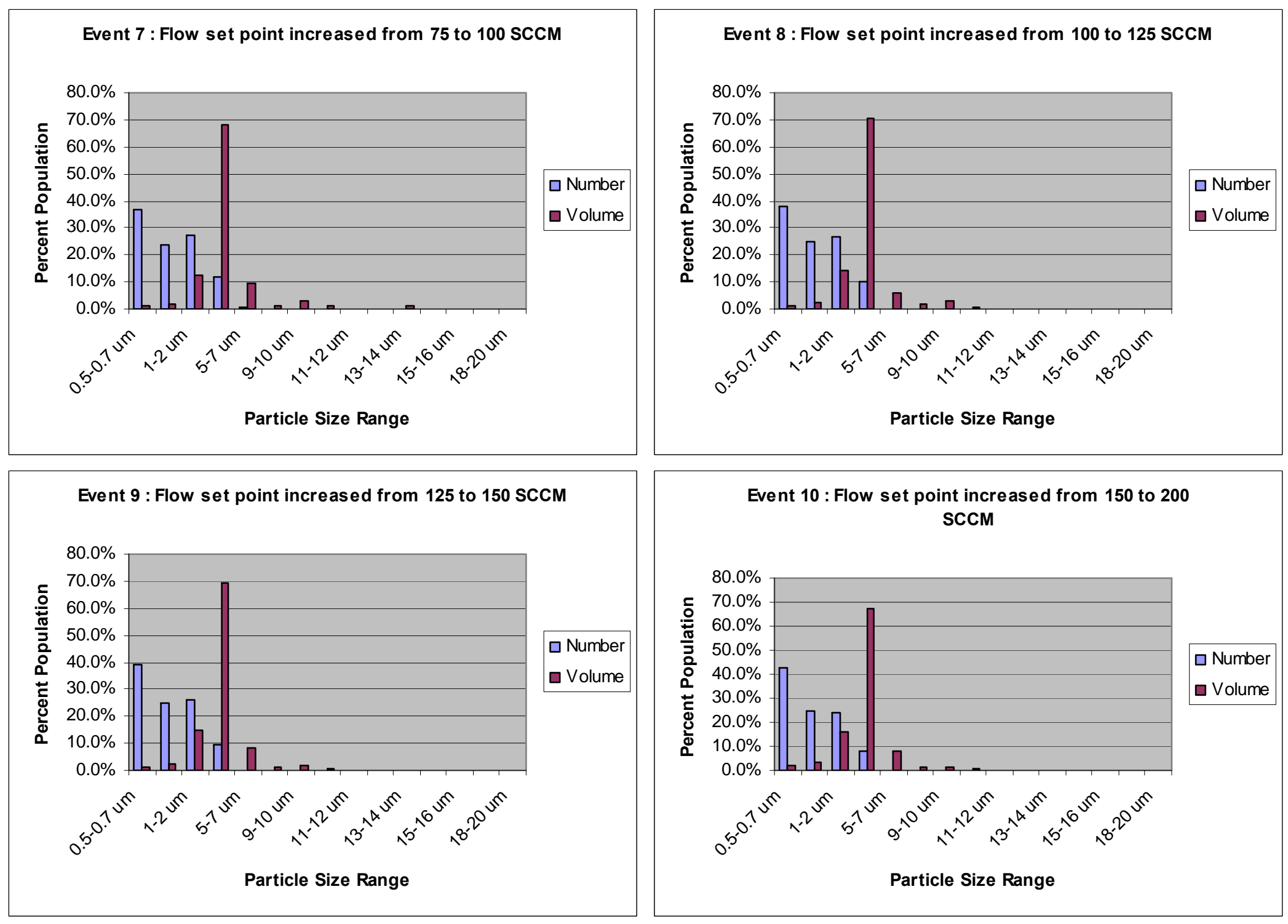

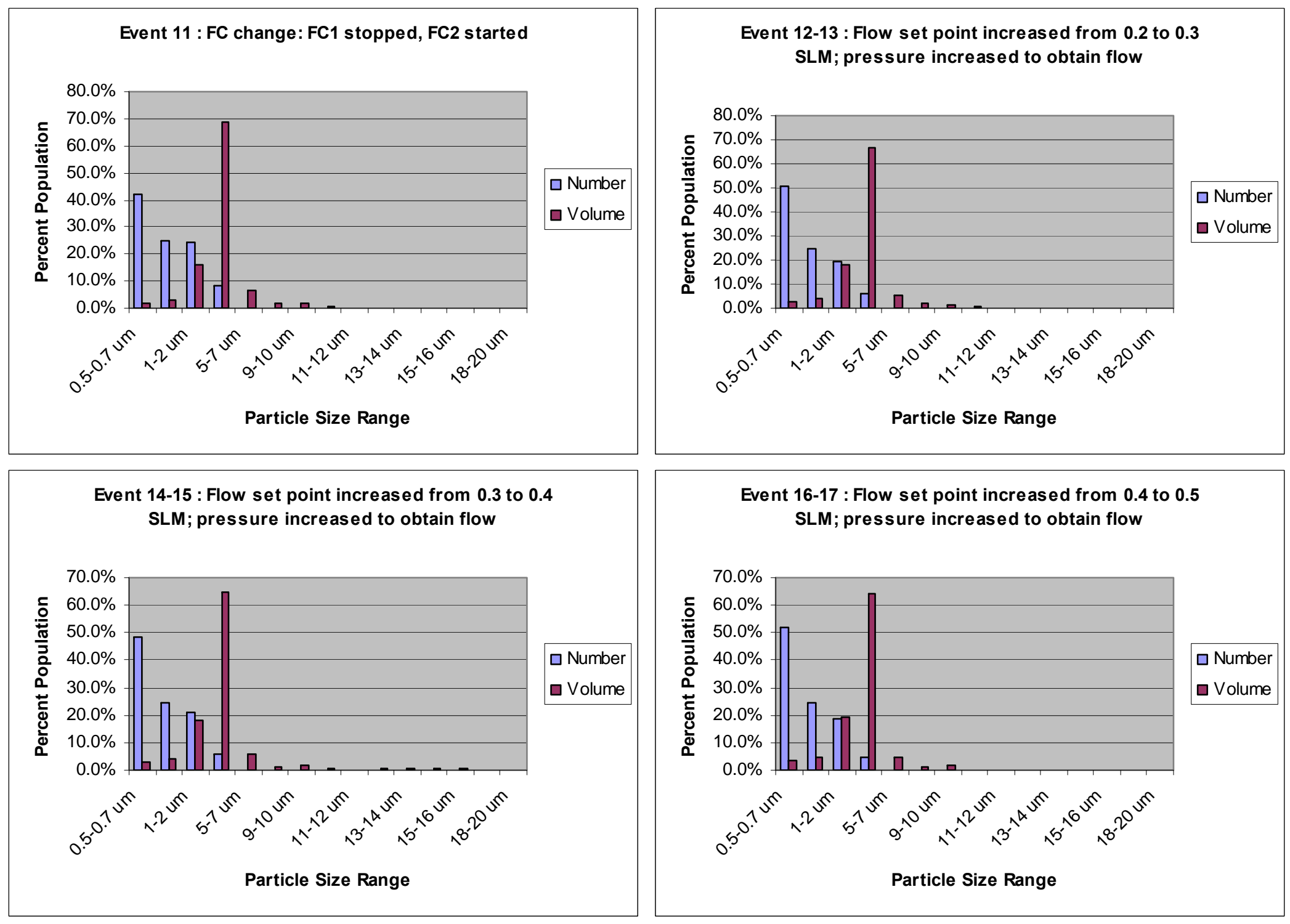

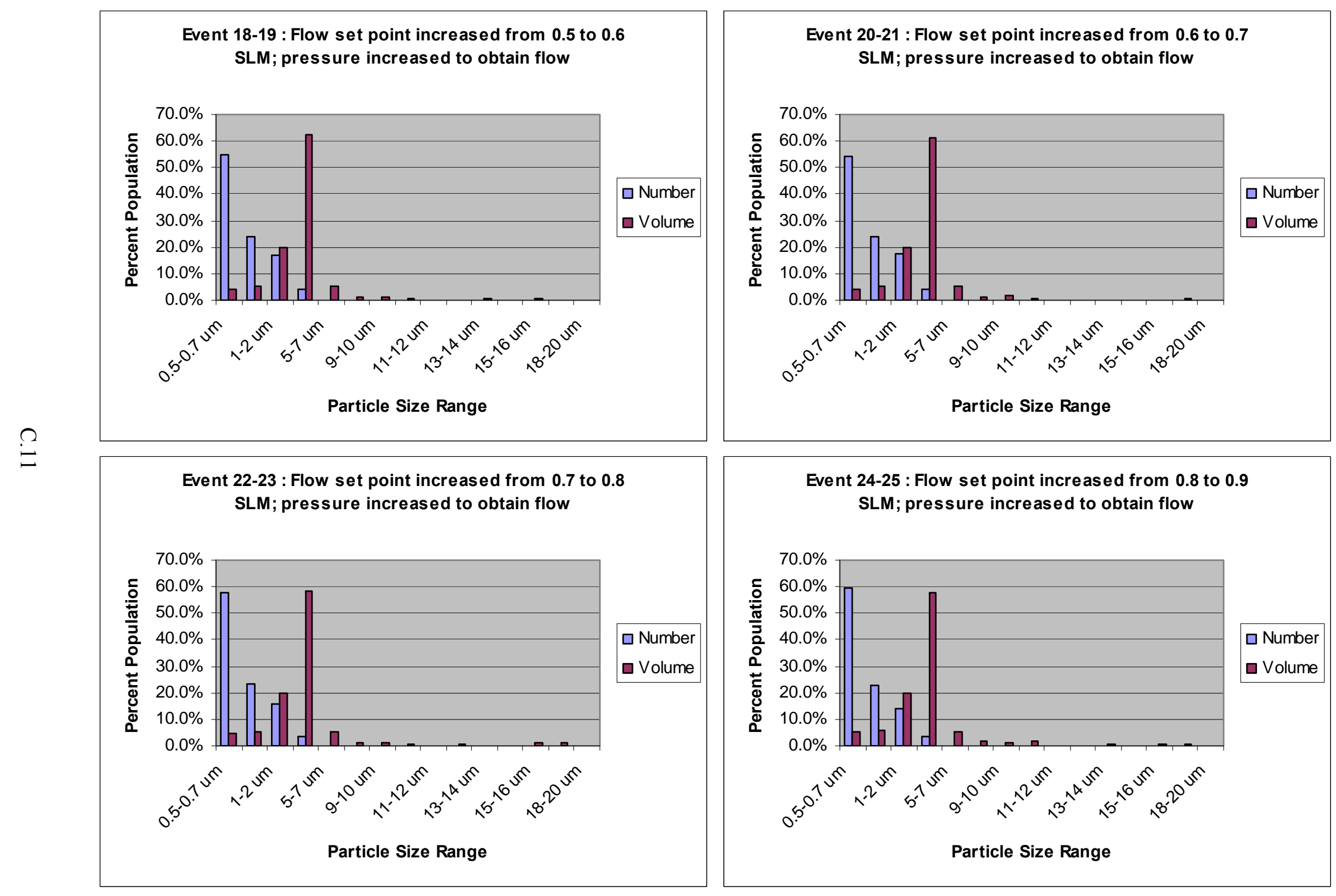

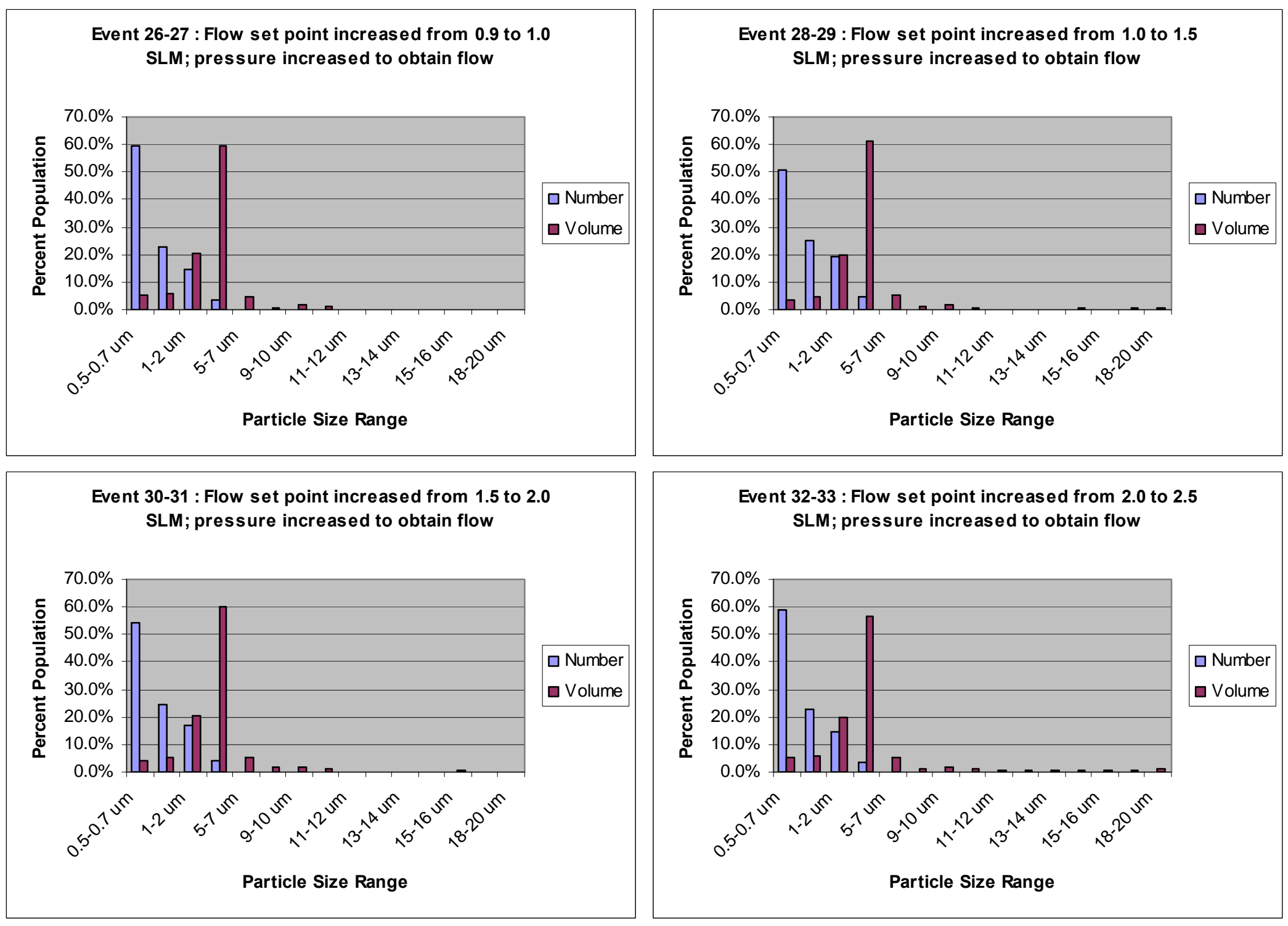

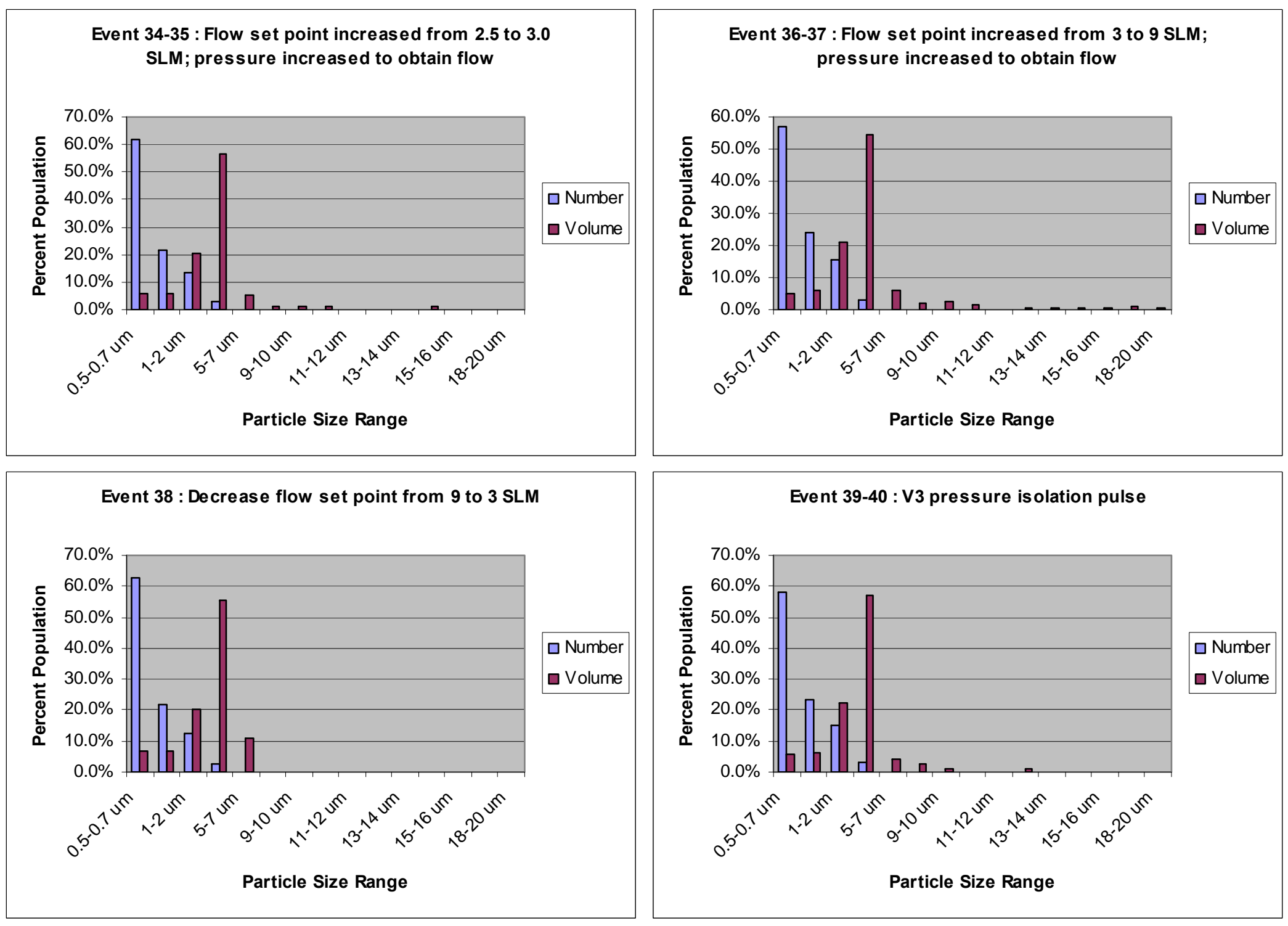

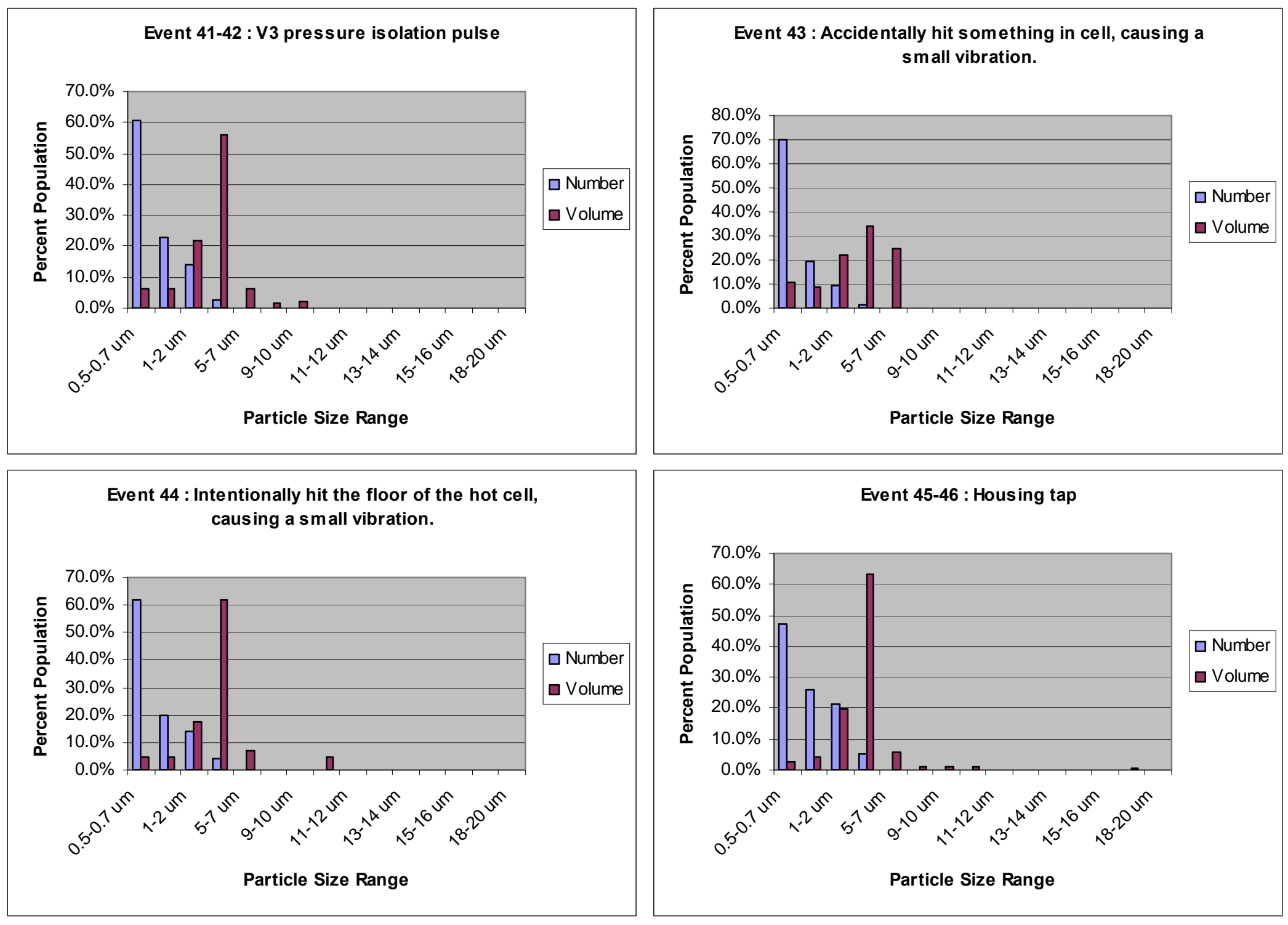
ATM106 Test 2
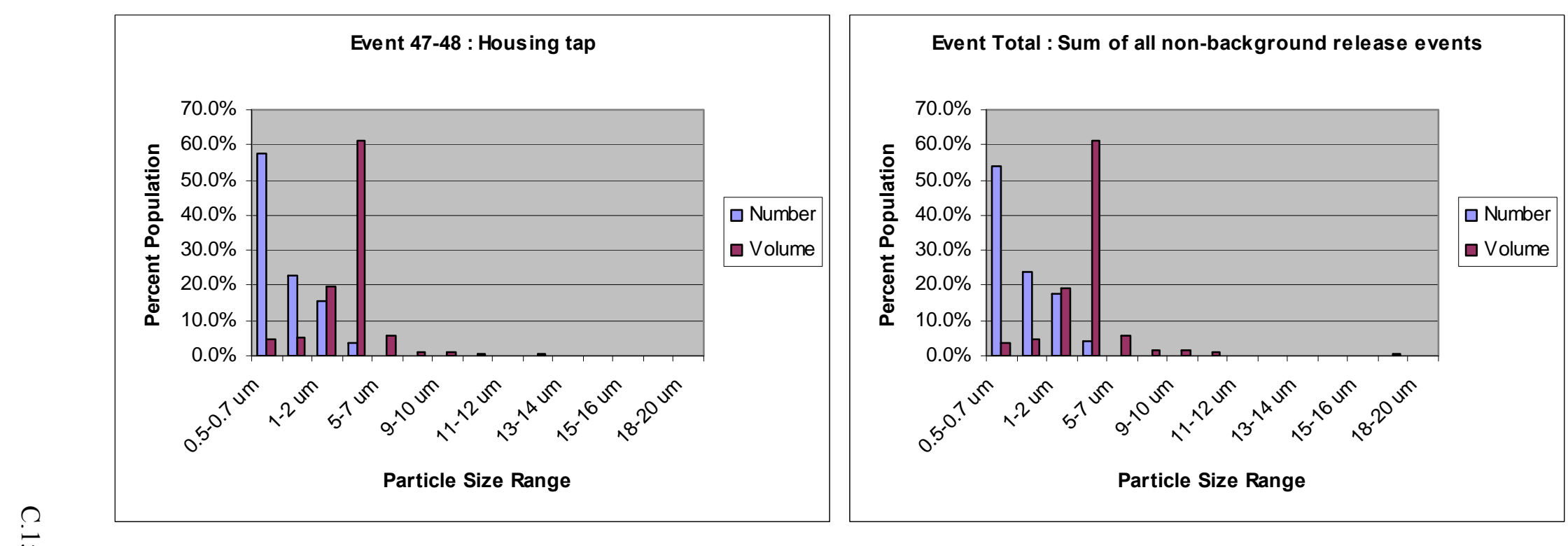
Event 2 : Increased flow set point from 10 to 30 SCCM
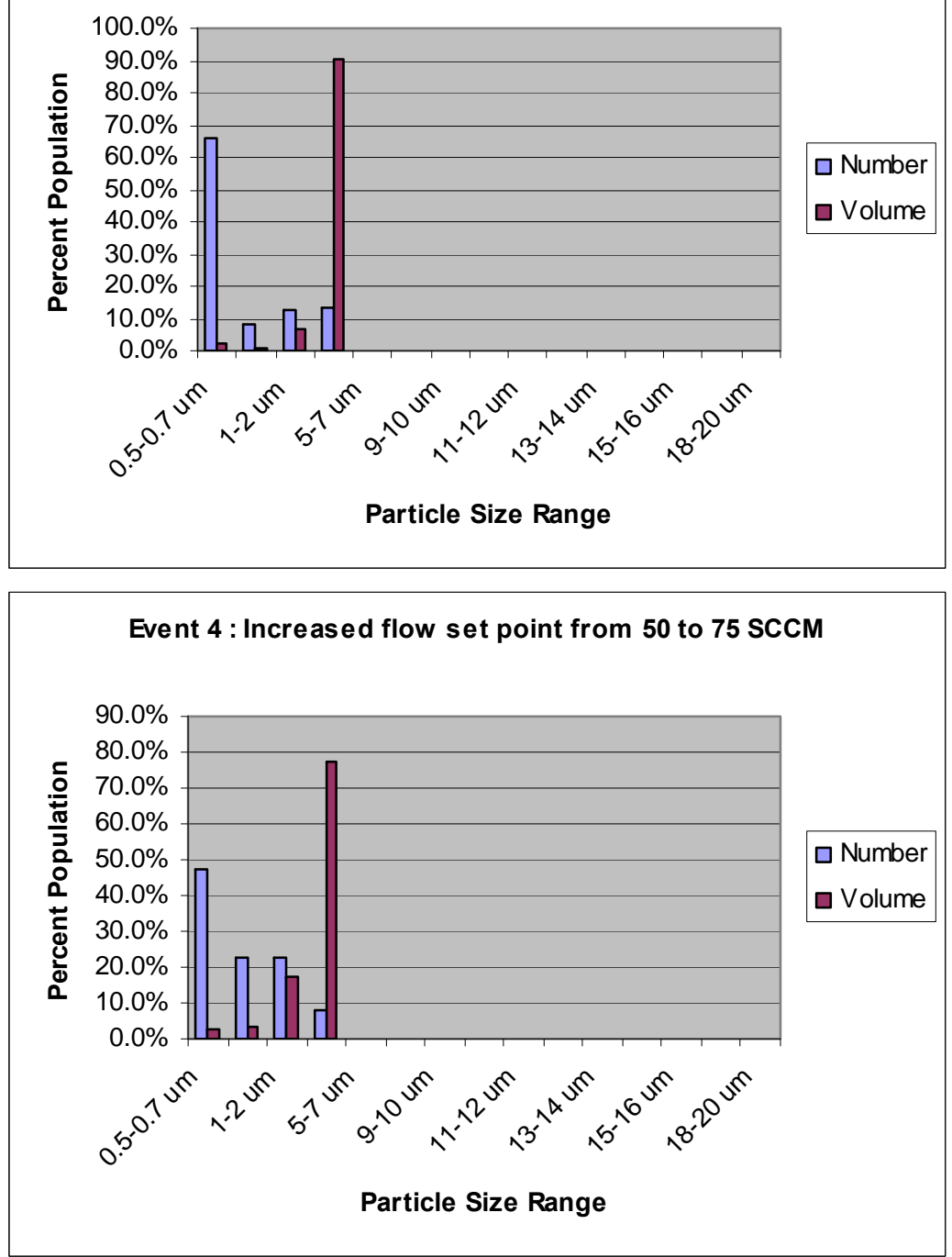

Event 3 : Increased flow set point from 30 to 50 SCCM

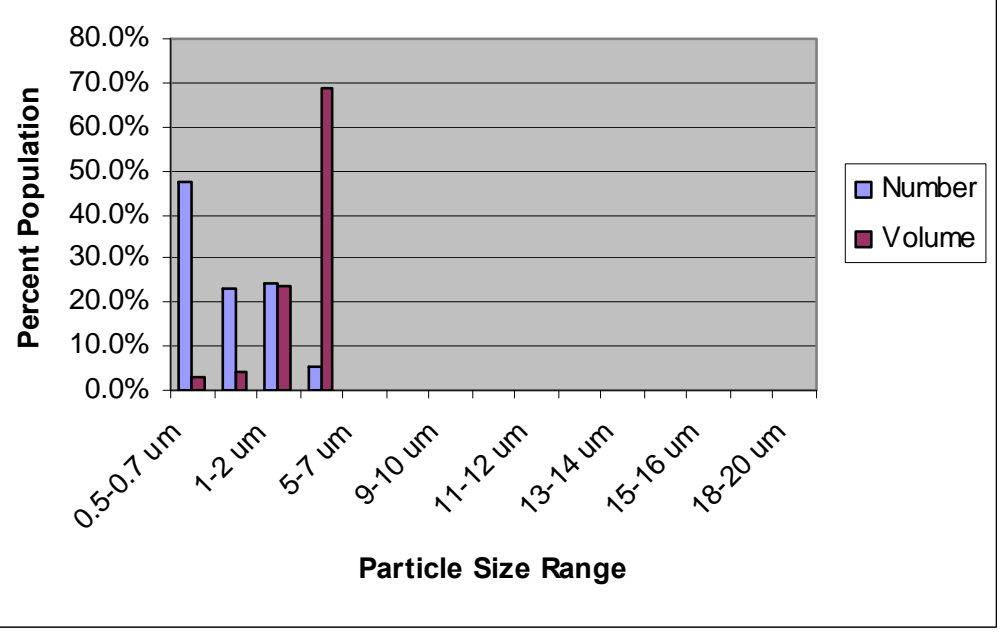

Event 5 : Increased flow set point from 75 to 100 SCCM

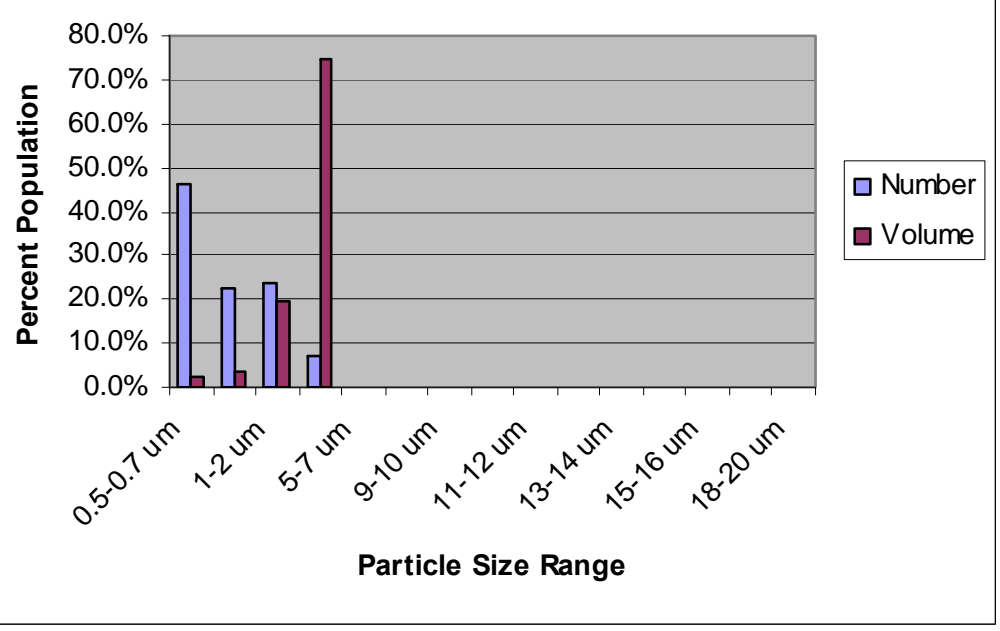



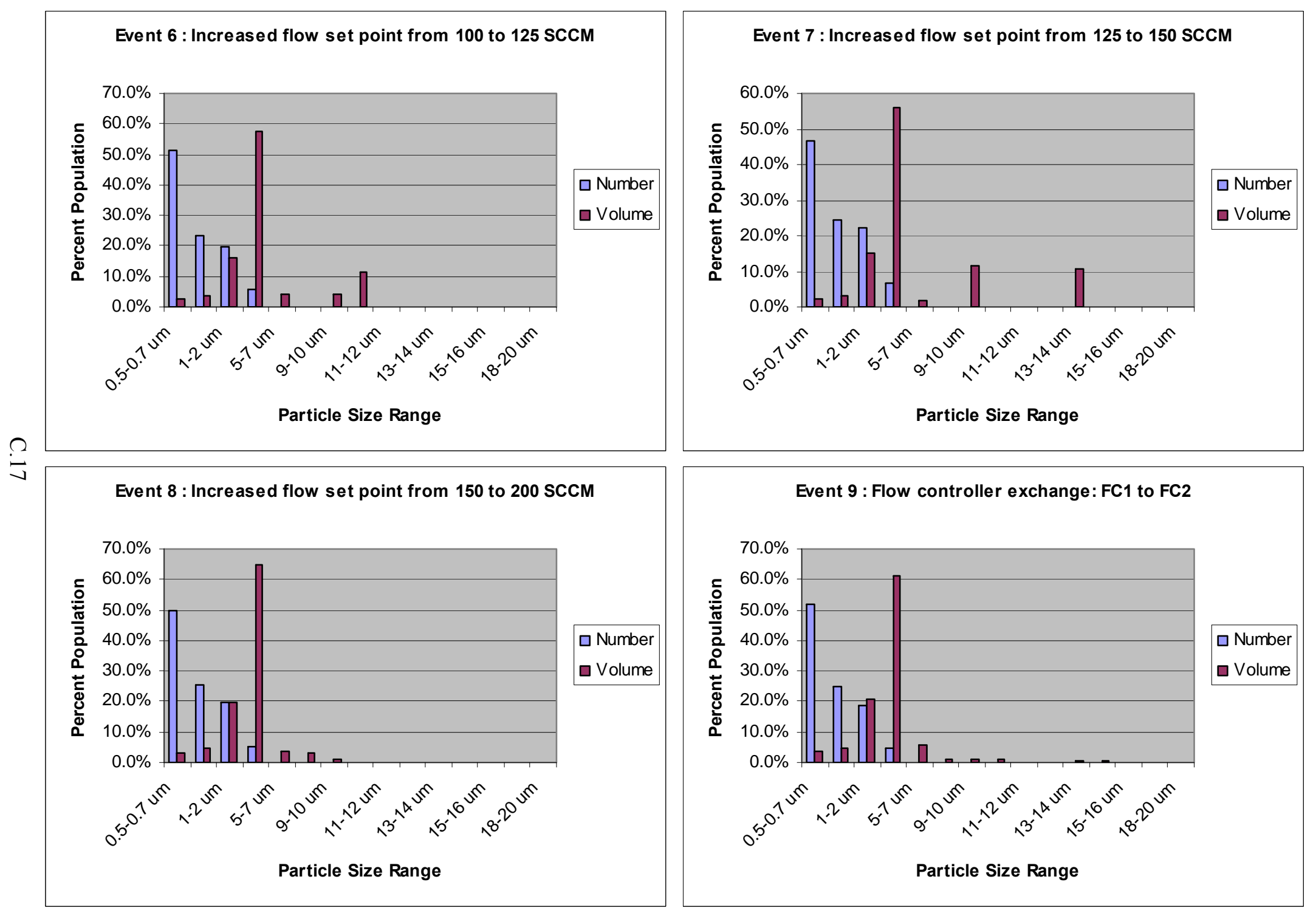
Event 10 : Increased flow set point from 200 to 300 SCCM

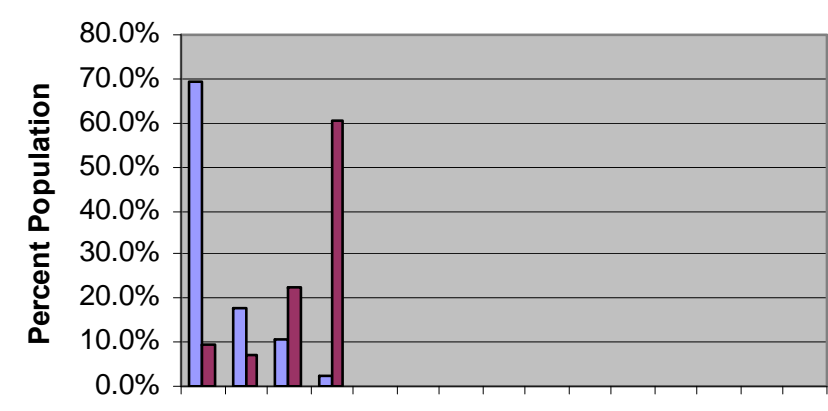

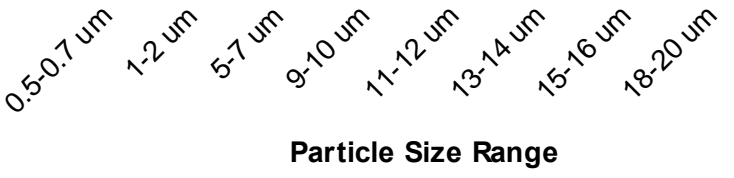

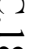

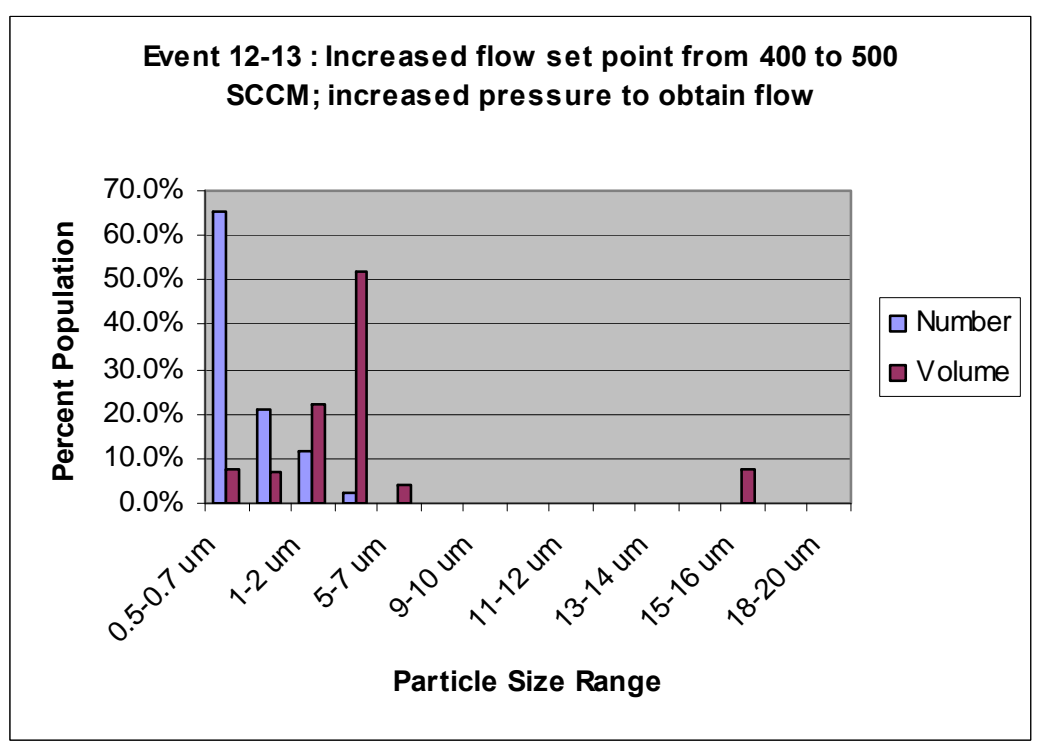

Event 11 : Increased flow set point from 300 to 400 SCCM

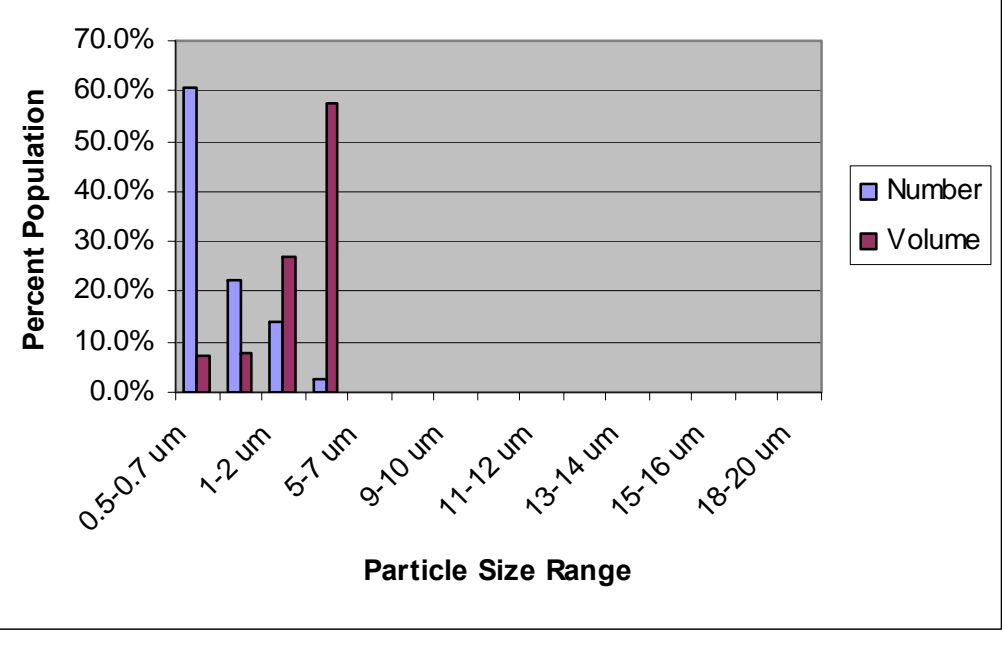

Event 14-15: Increased flow set point from 500 to 600 SCCM; increased pressure to obtain flow

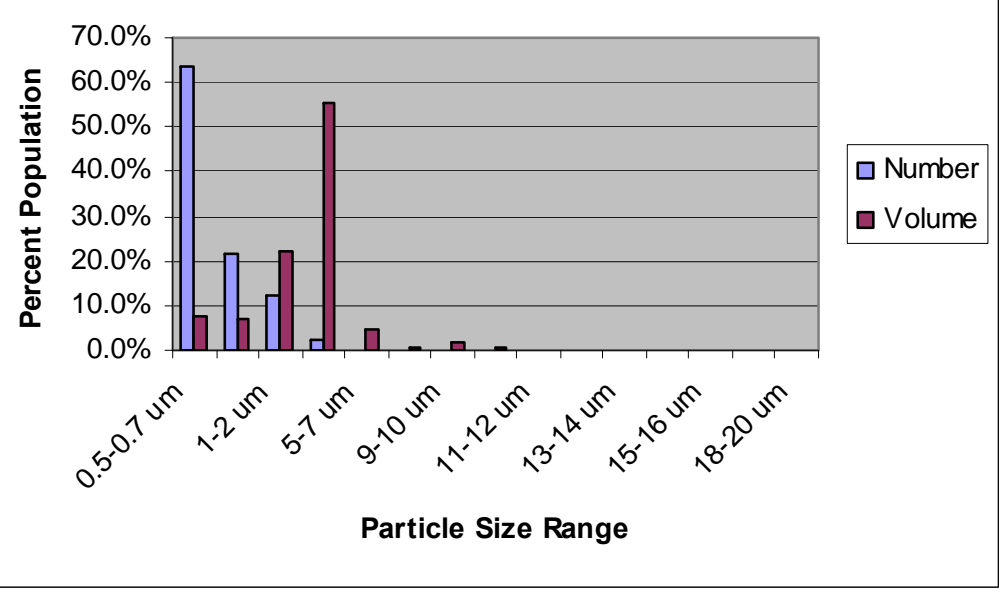



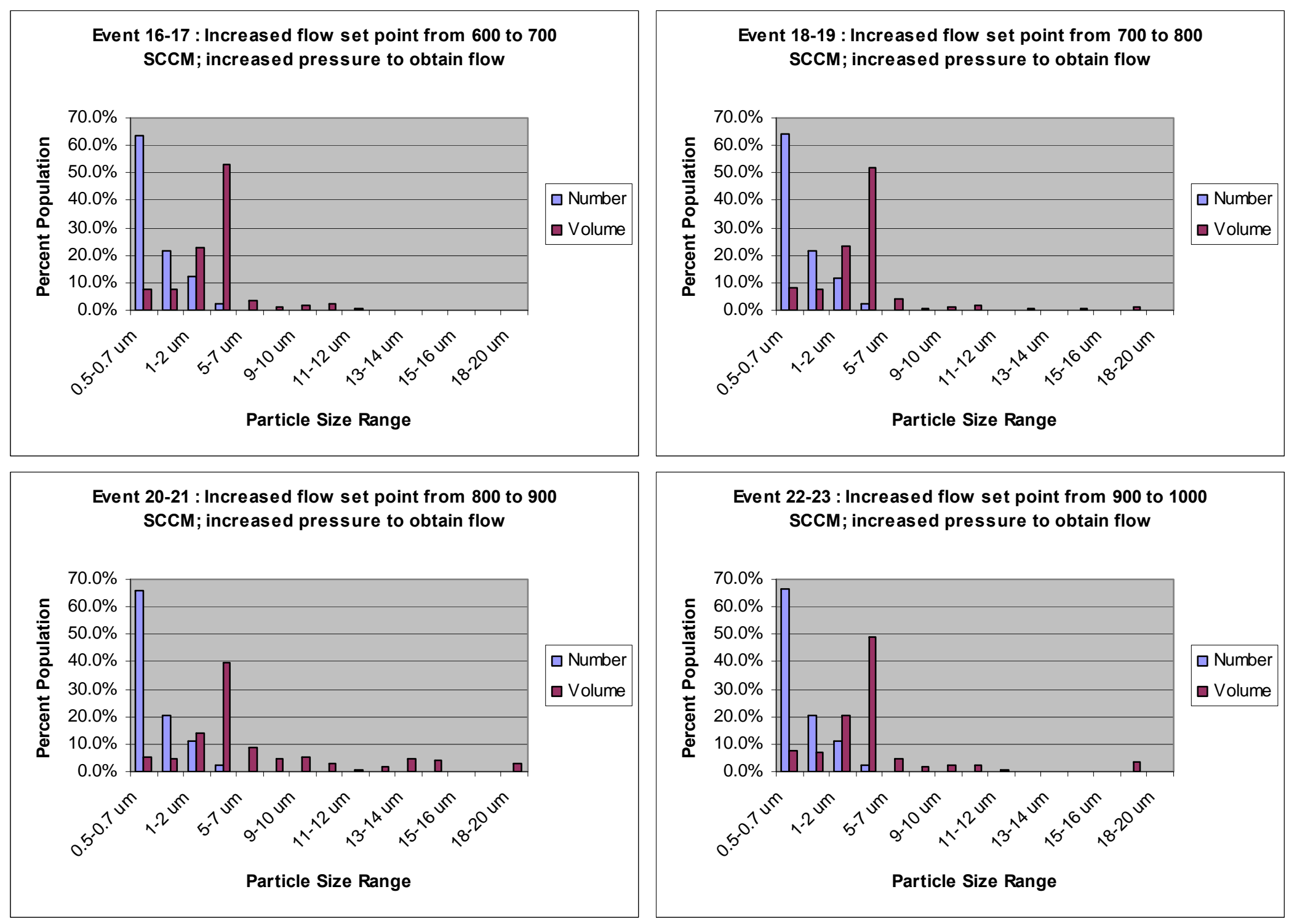
Event 24-25 : Increased flow set point from 1.0 to 1.5 SLM; increased pressure to obtain flow

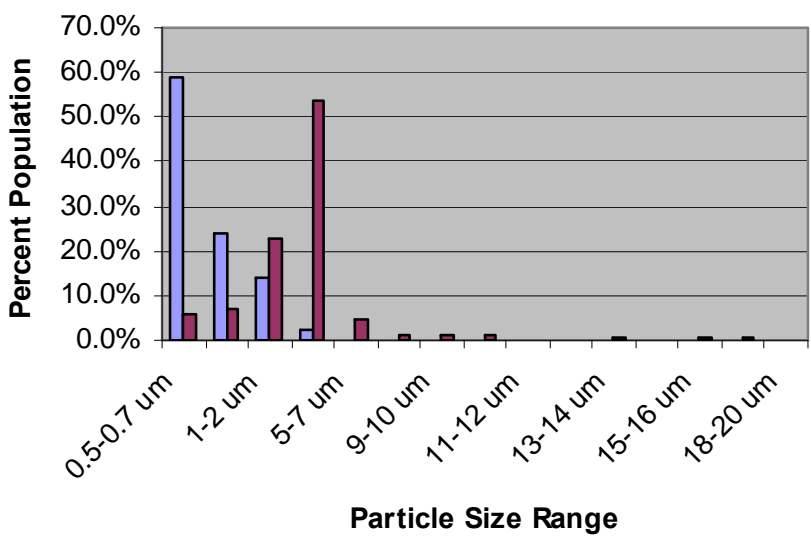

ก

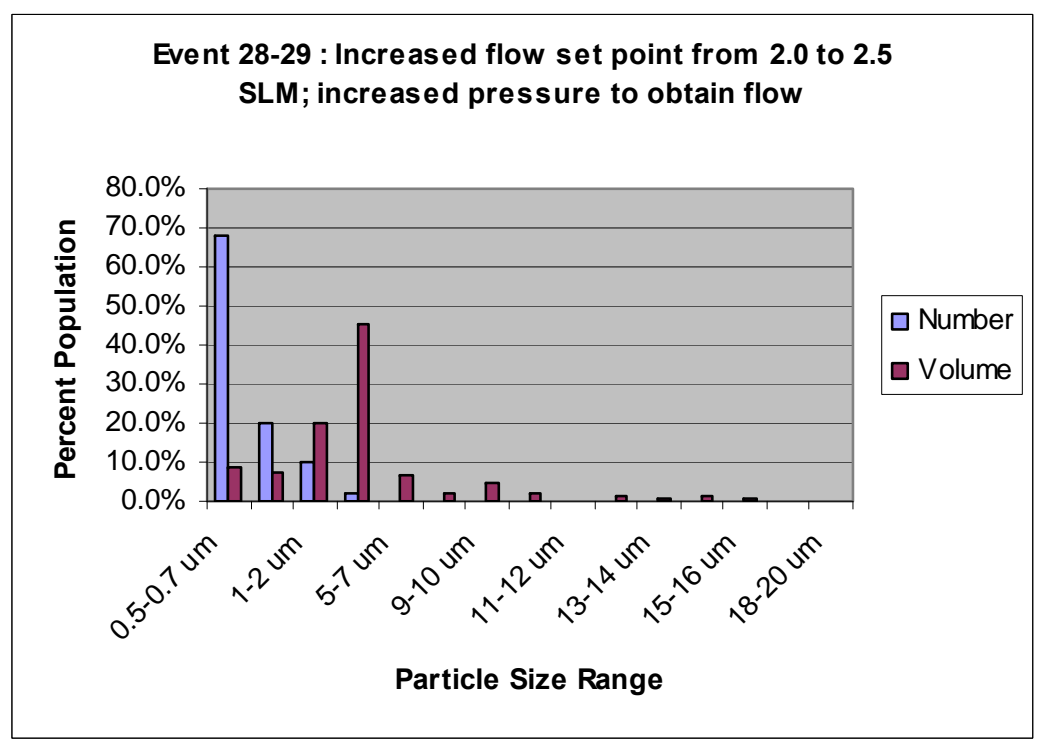

Event 26-27 : Increased flow set point from 1.5 to 2.0 SLM; increased pressure to obtain flow

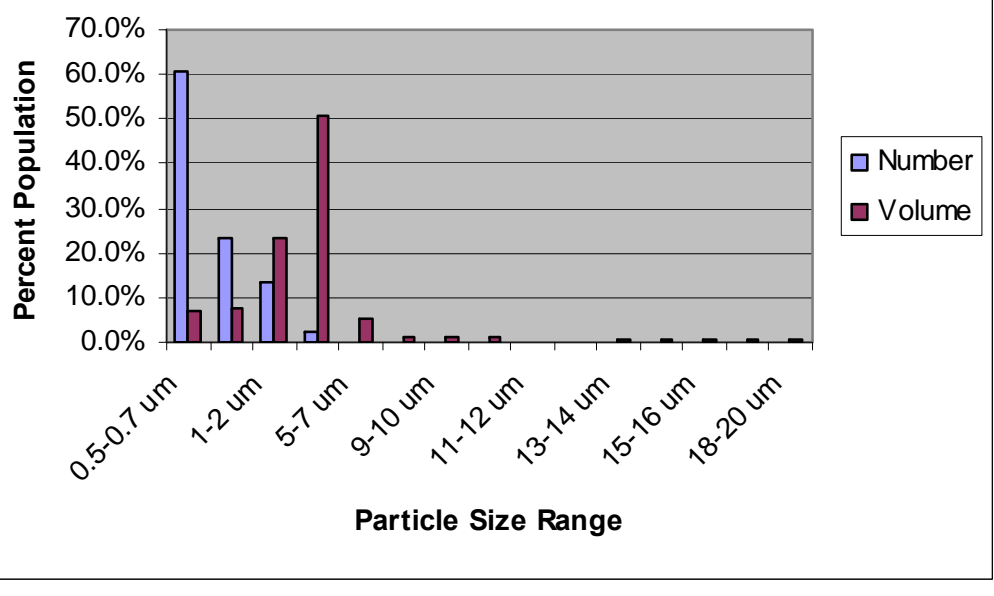

Event 30-31: Increased flow set point from 2.5 to 3.0 SLM; increased pressure to obtain flow

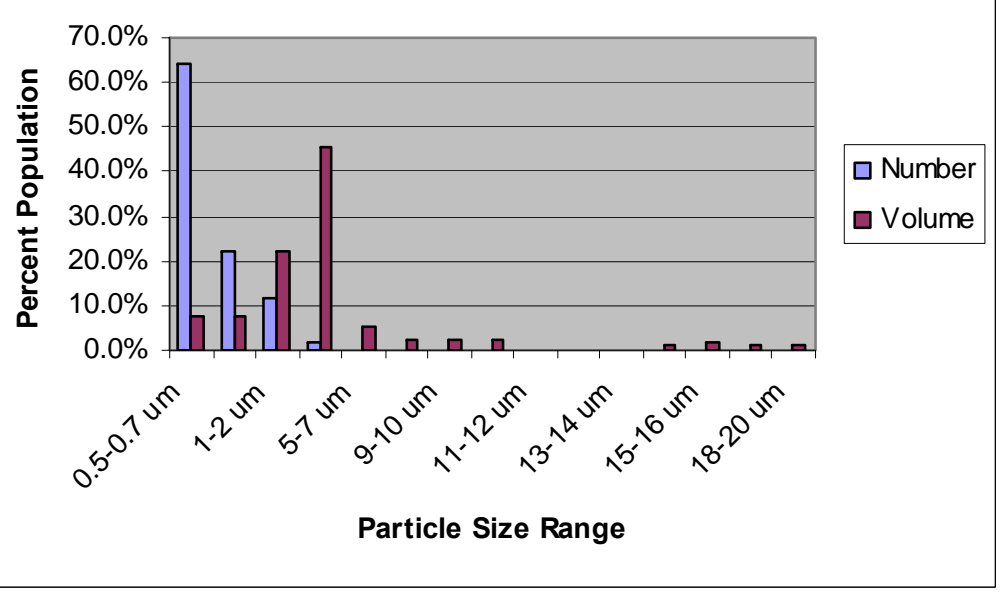



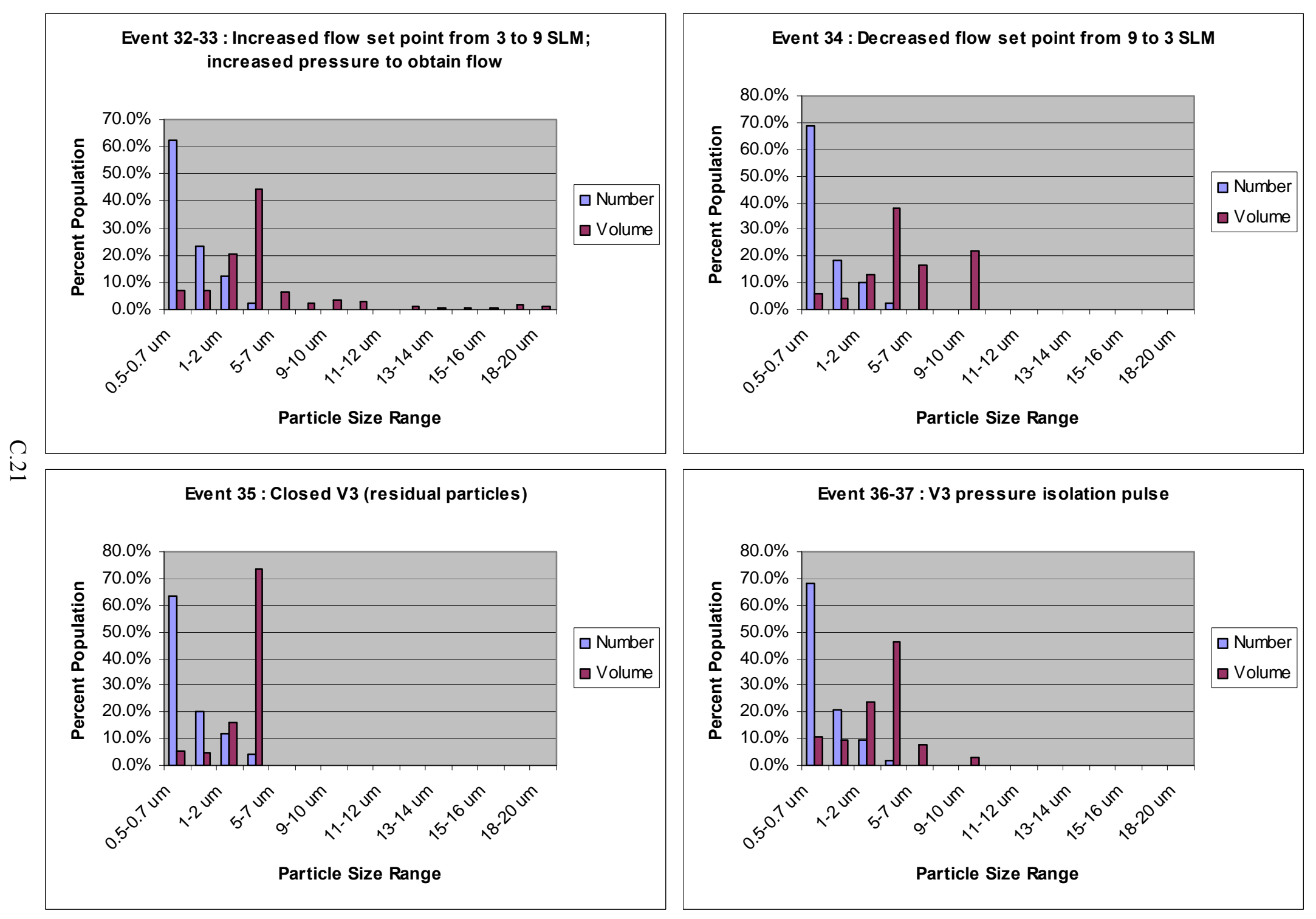

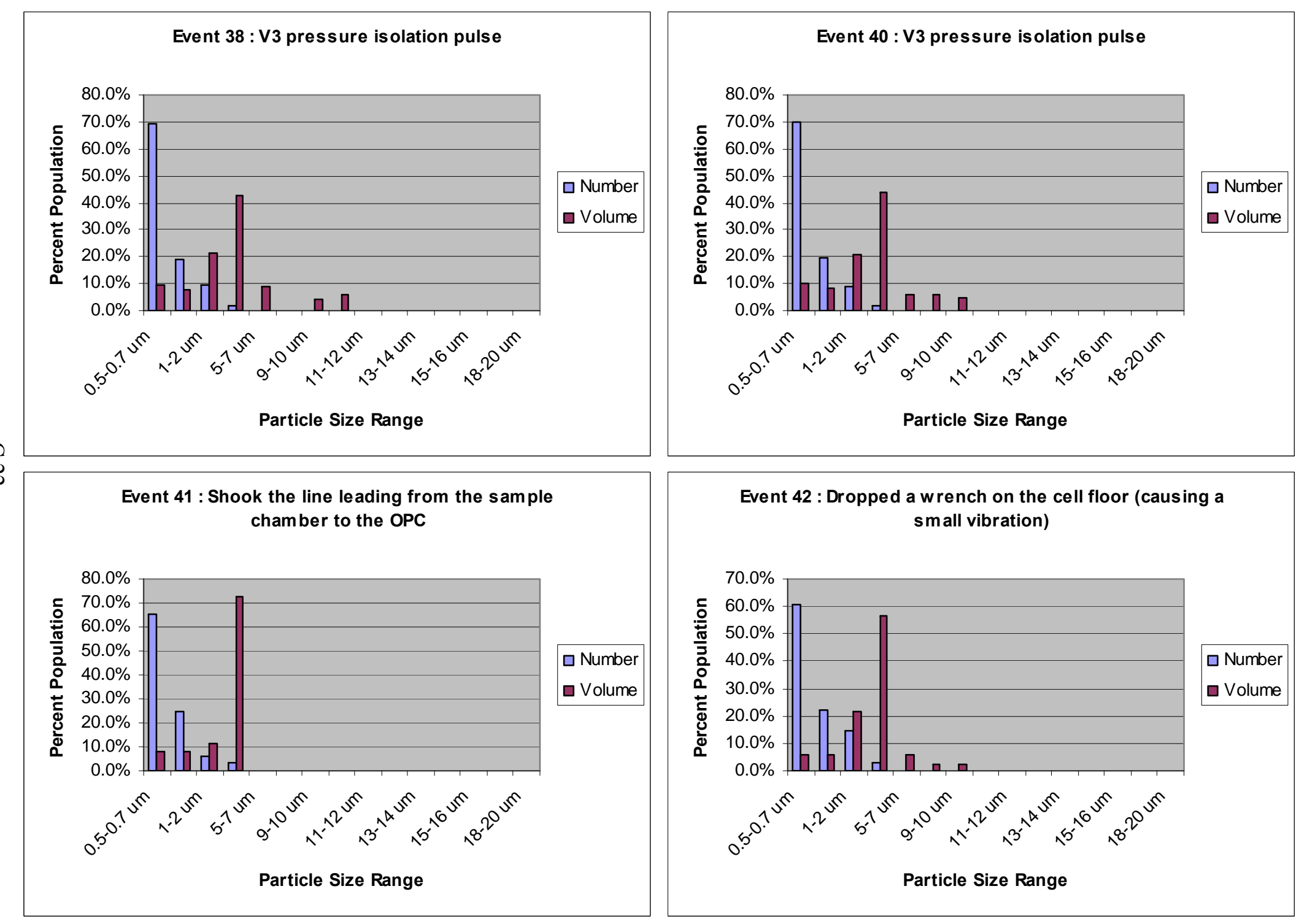

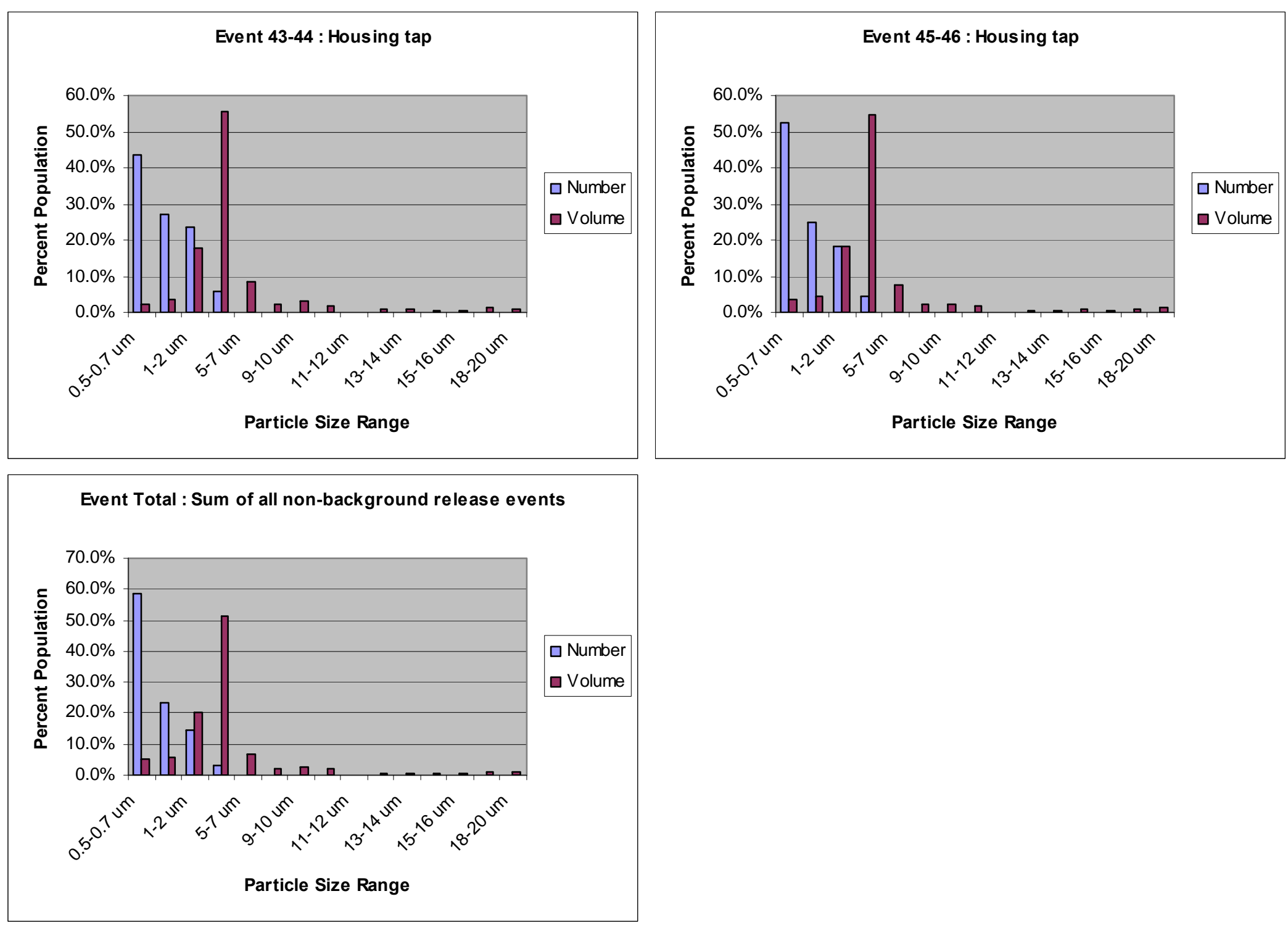
Event 3 : Increased flow set point from 50 to 100 SCCM; increased pressure to obtain flow

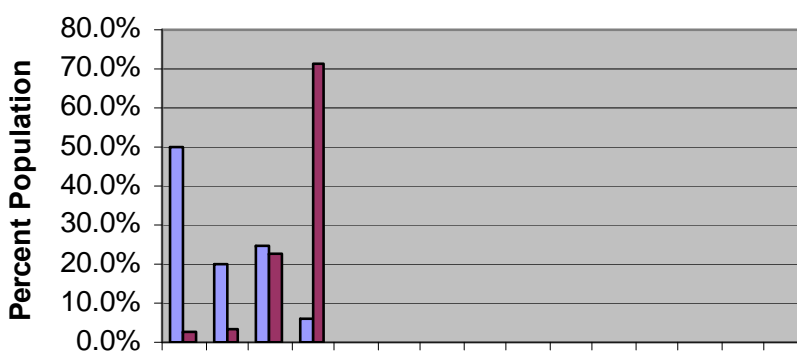

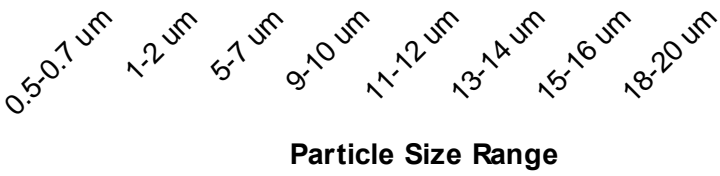

N

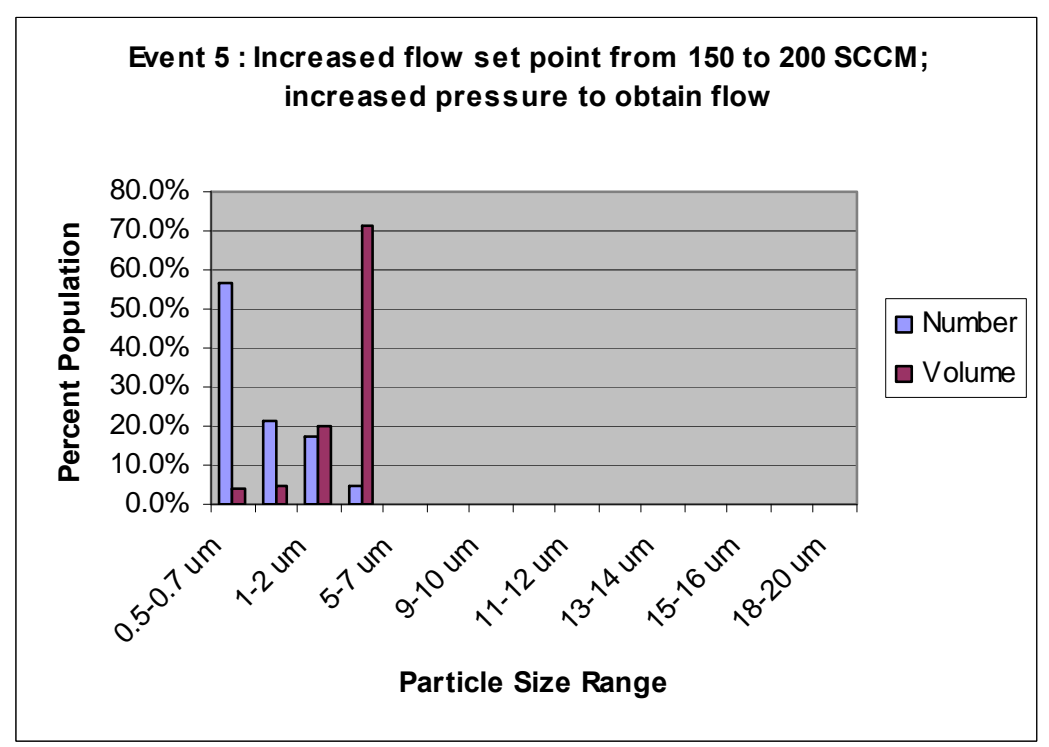

Event 4 : Increased flow set point from 100 to 150 SCCM; increased pressure to obtain flow

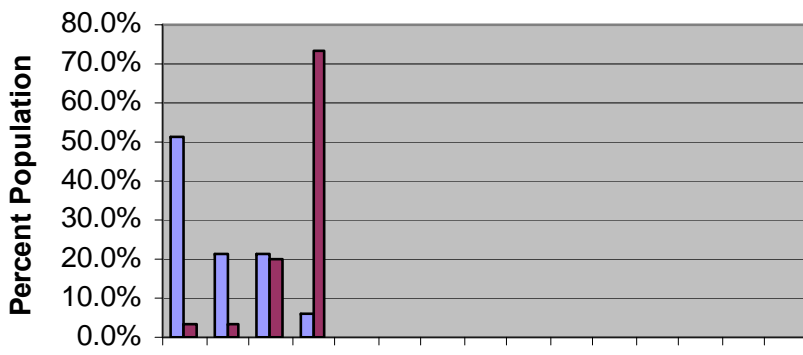

$\square$ Number

$\square$ Volume

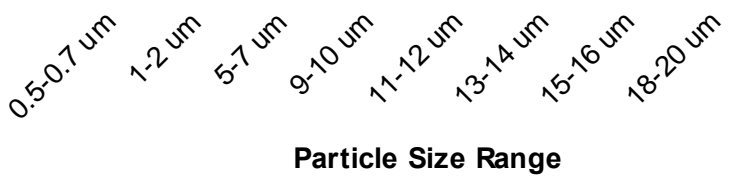

\section{Event 9 : Increased flow set point from 0 to 200 SCCM}

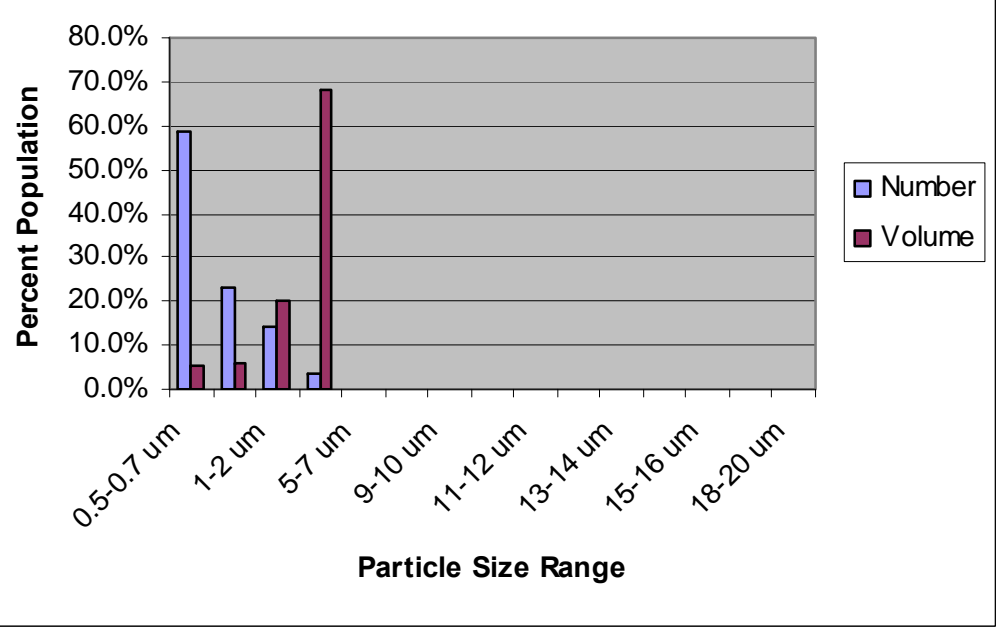



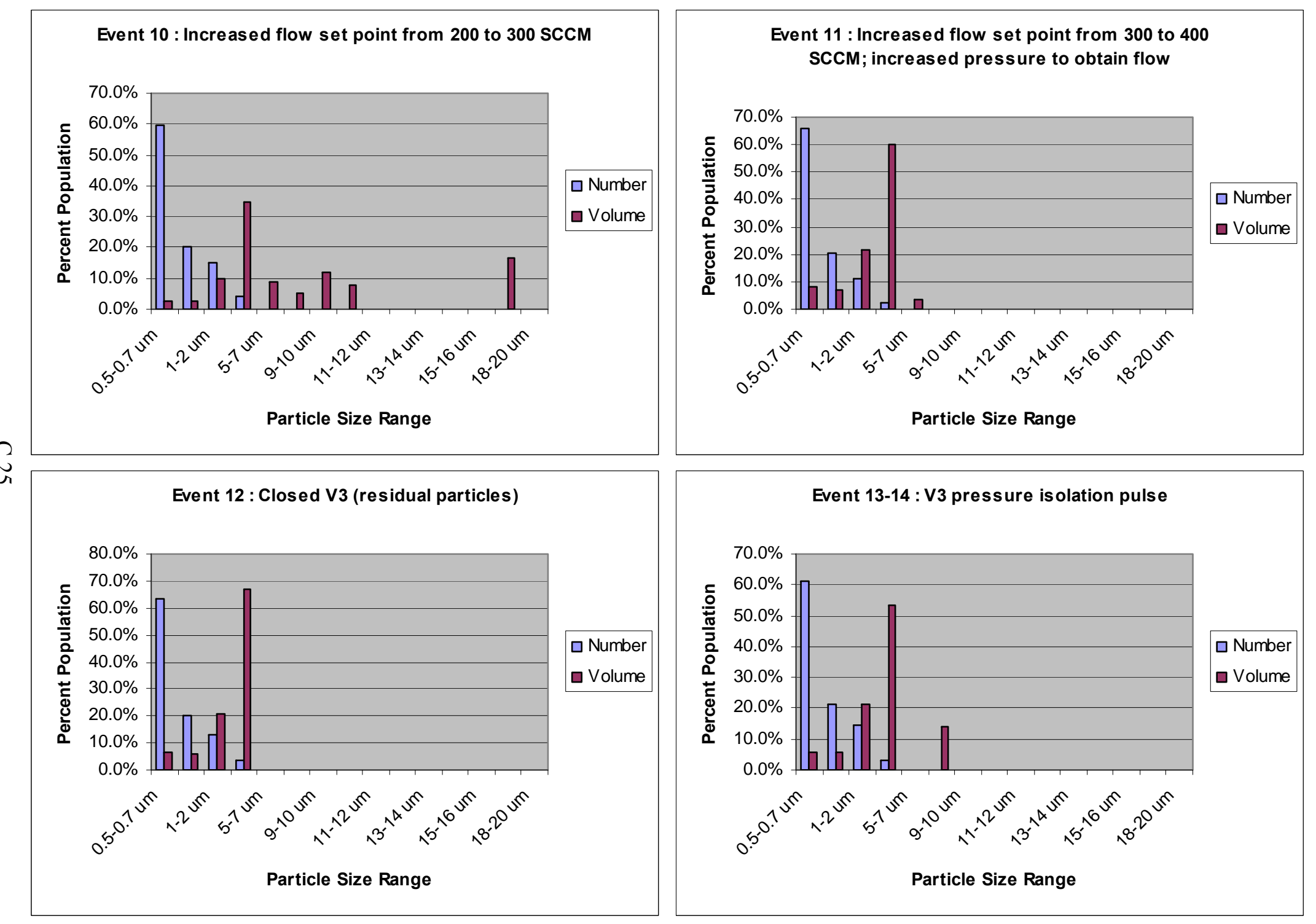

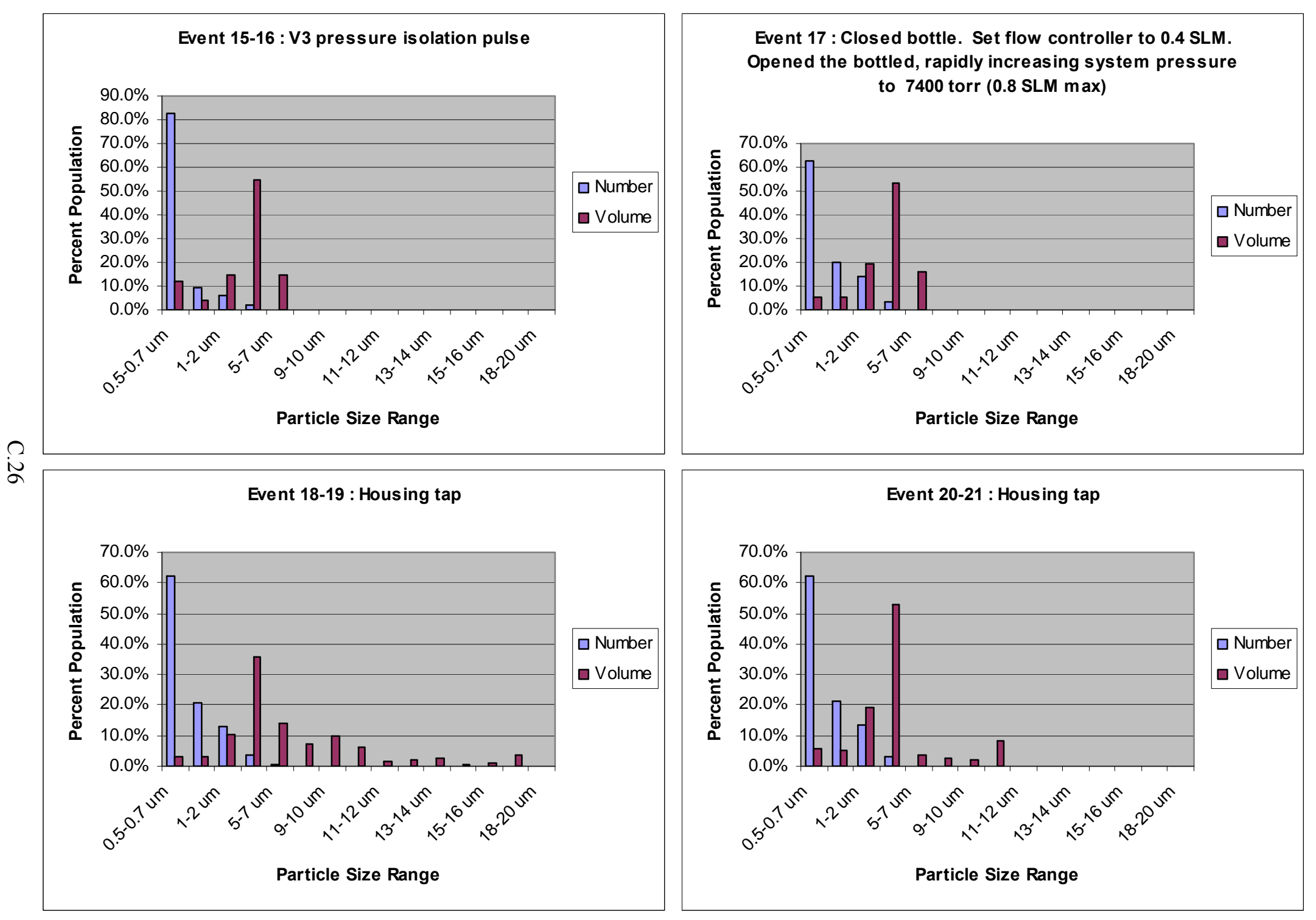
HBU-539B3 Test 1

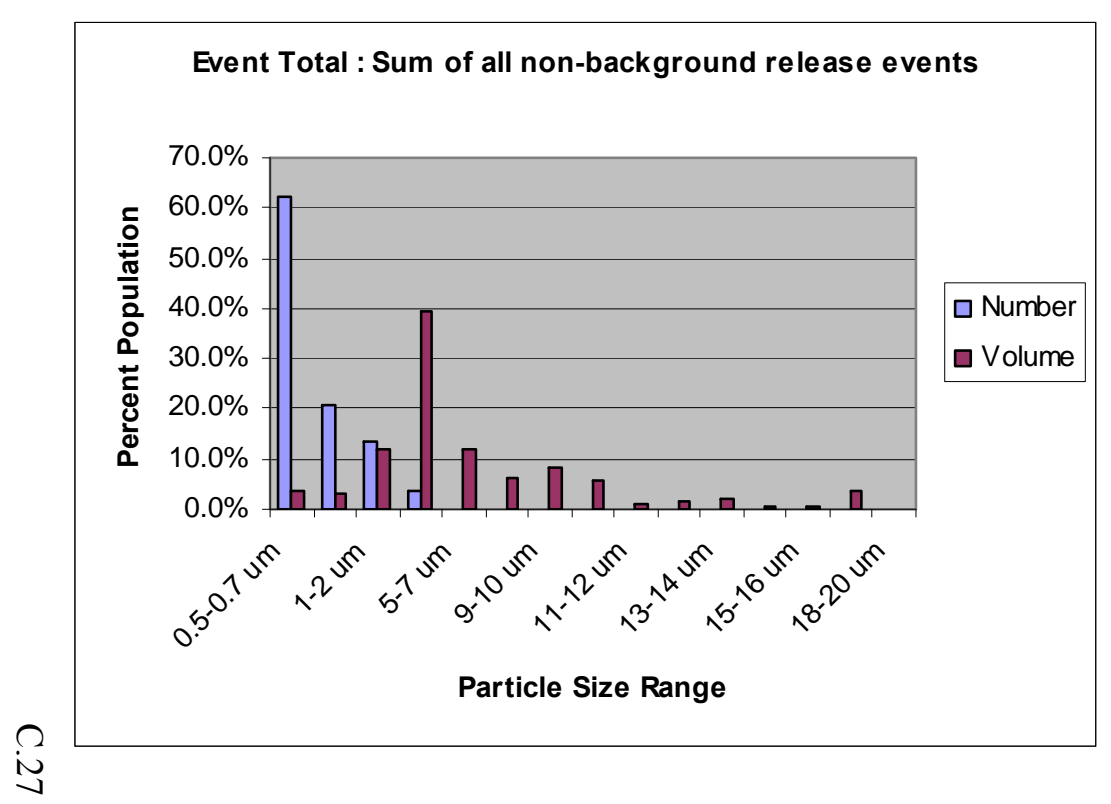



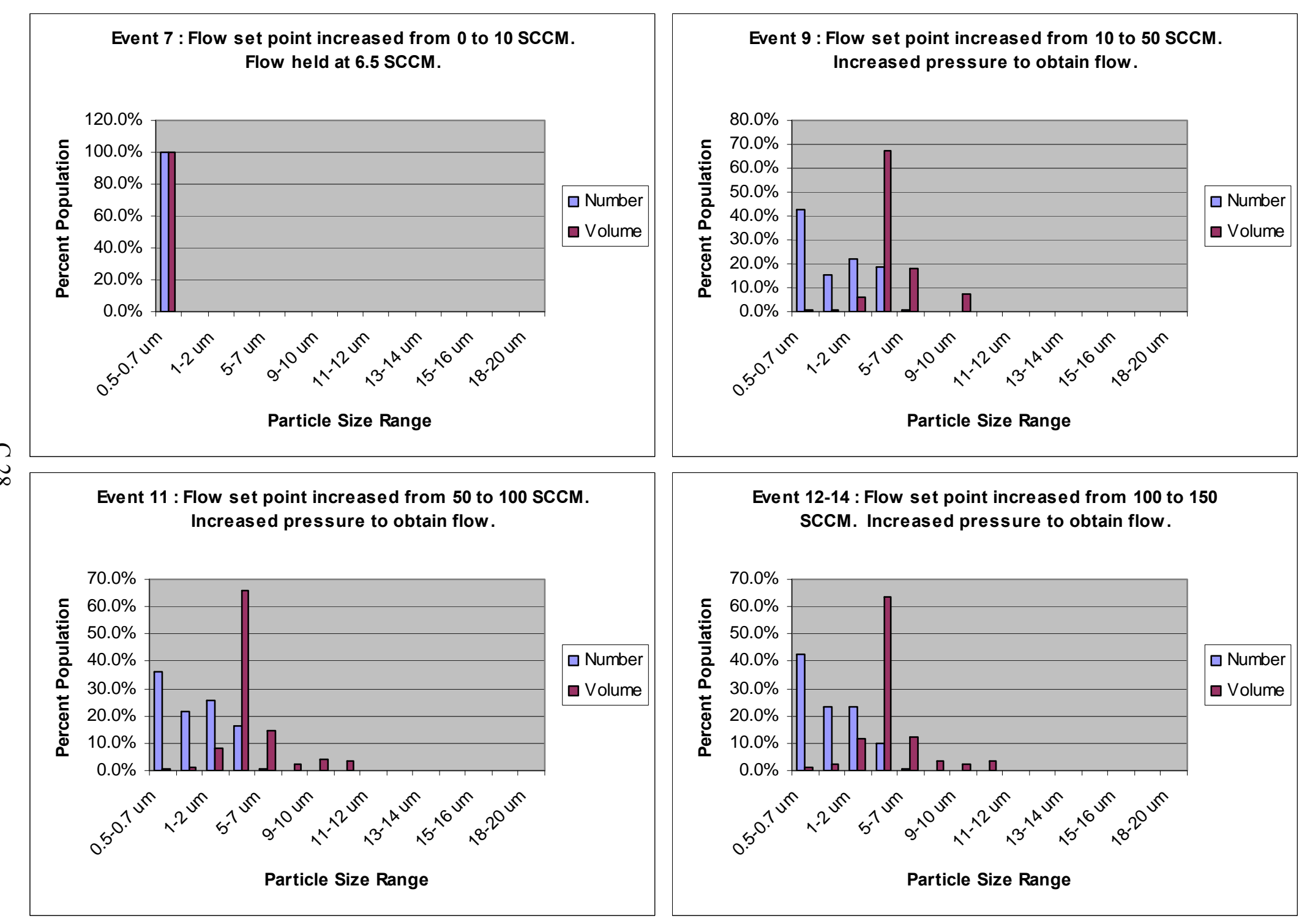

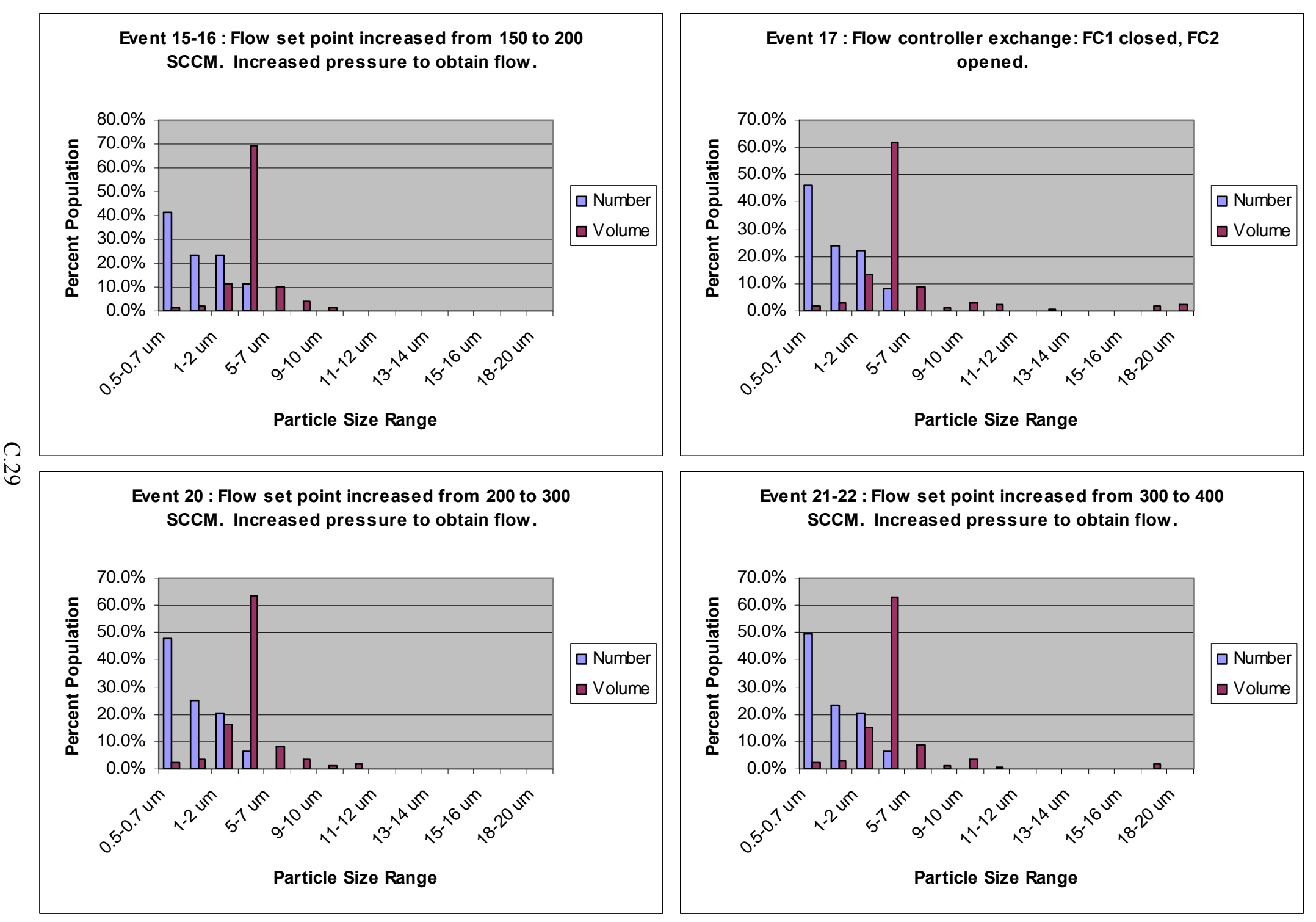

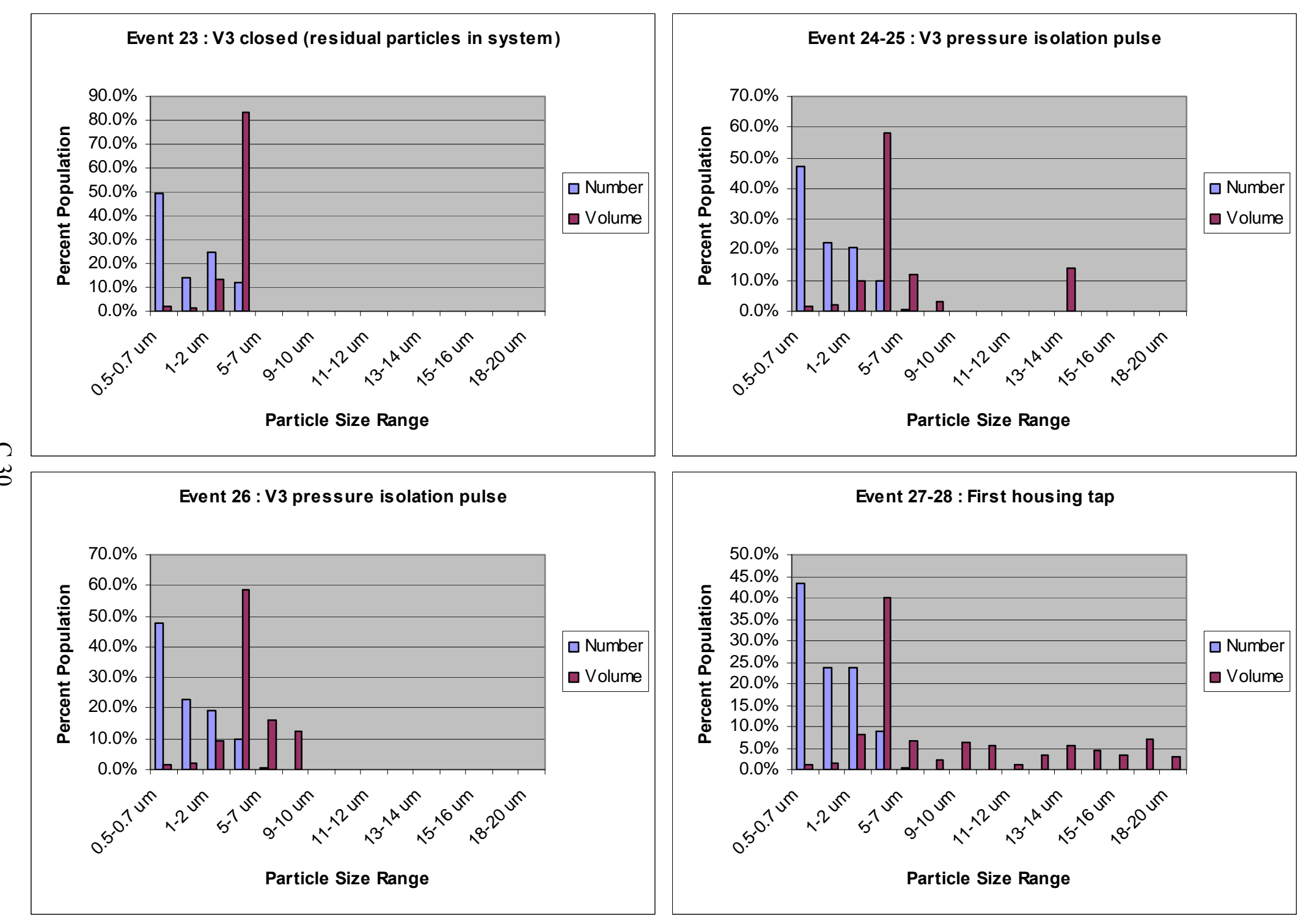
HBU-539B3 Test 2
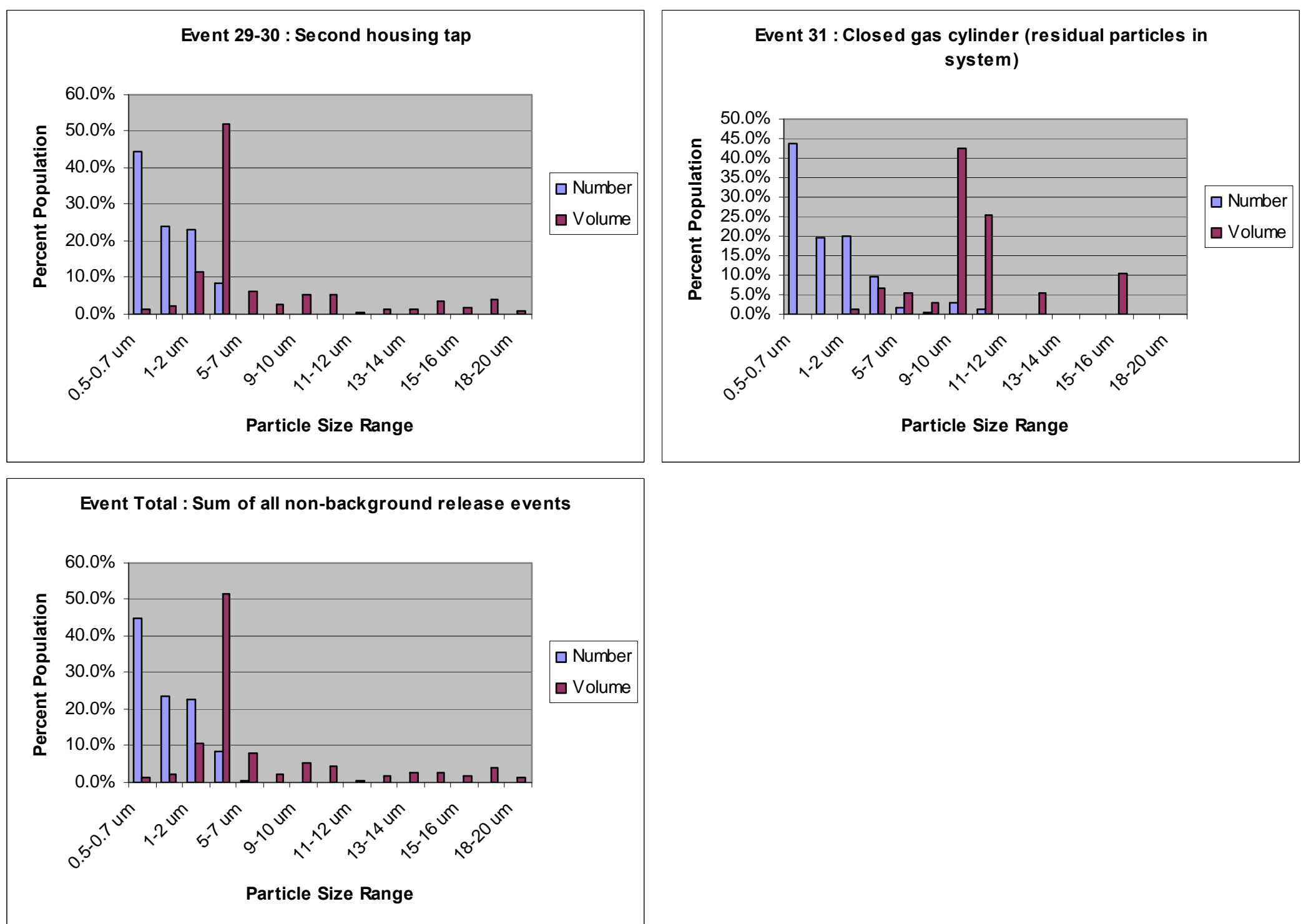

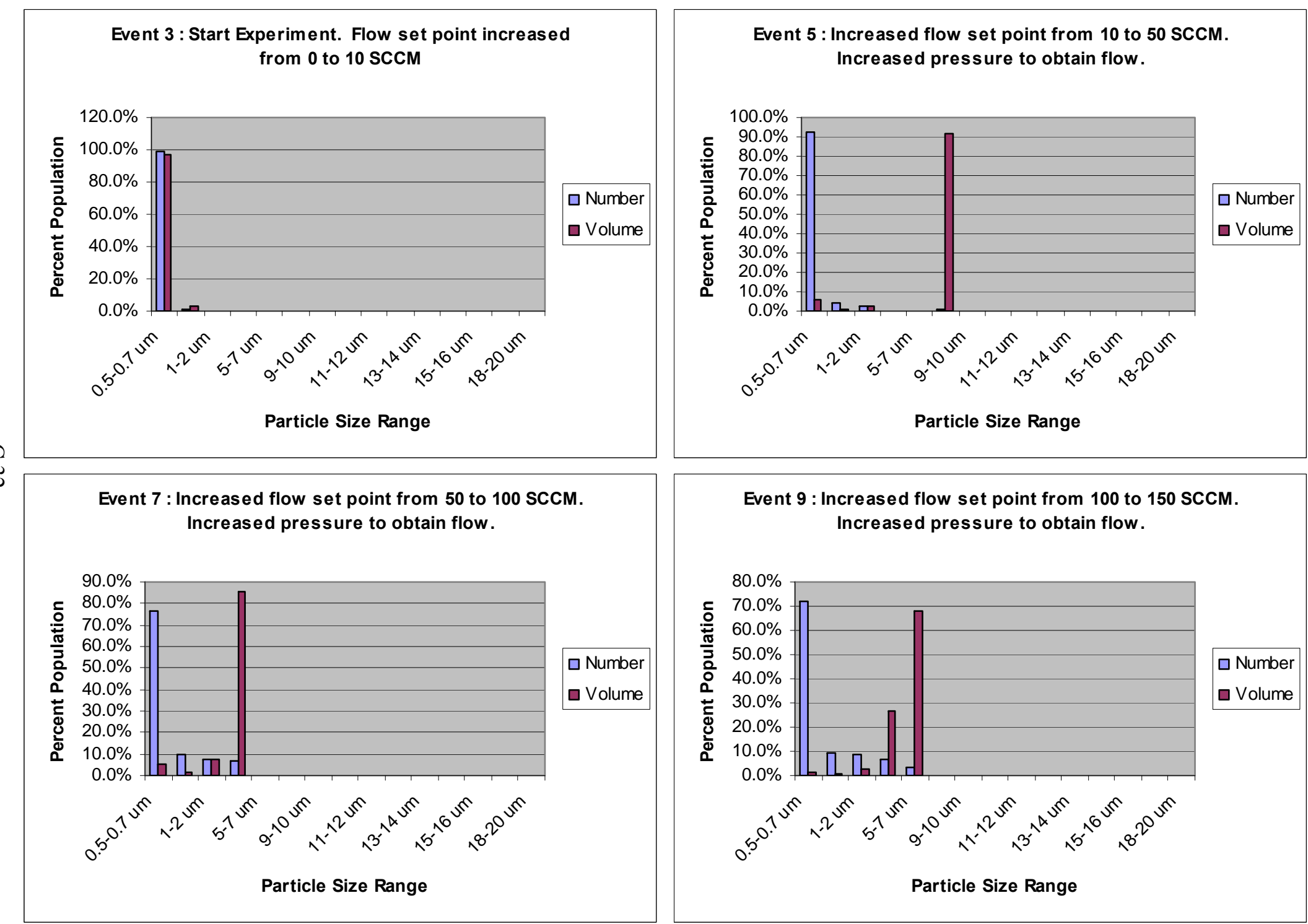

Event 9 : Increased flow set point from 100 to 150 SCCM. Increased pressure to obtain flow.

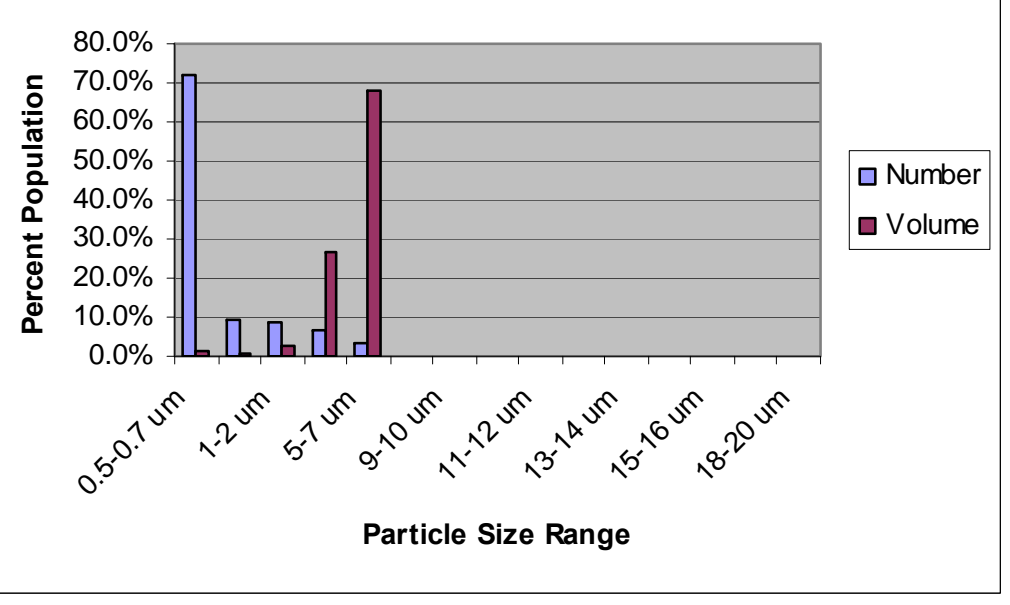



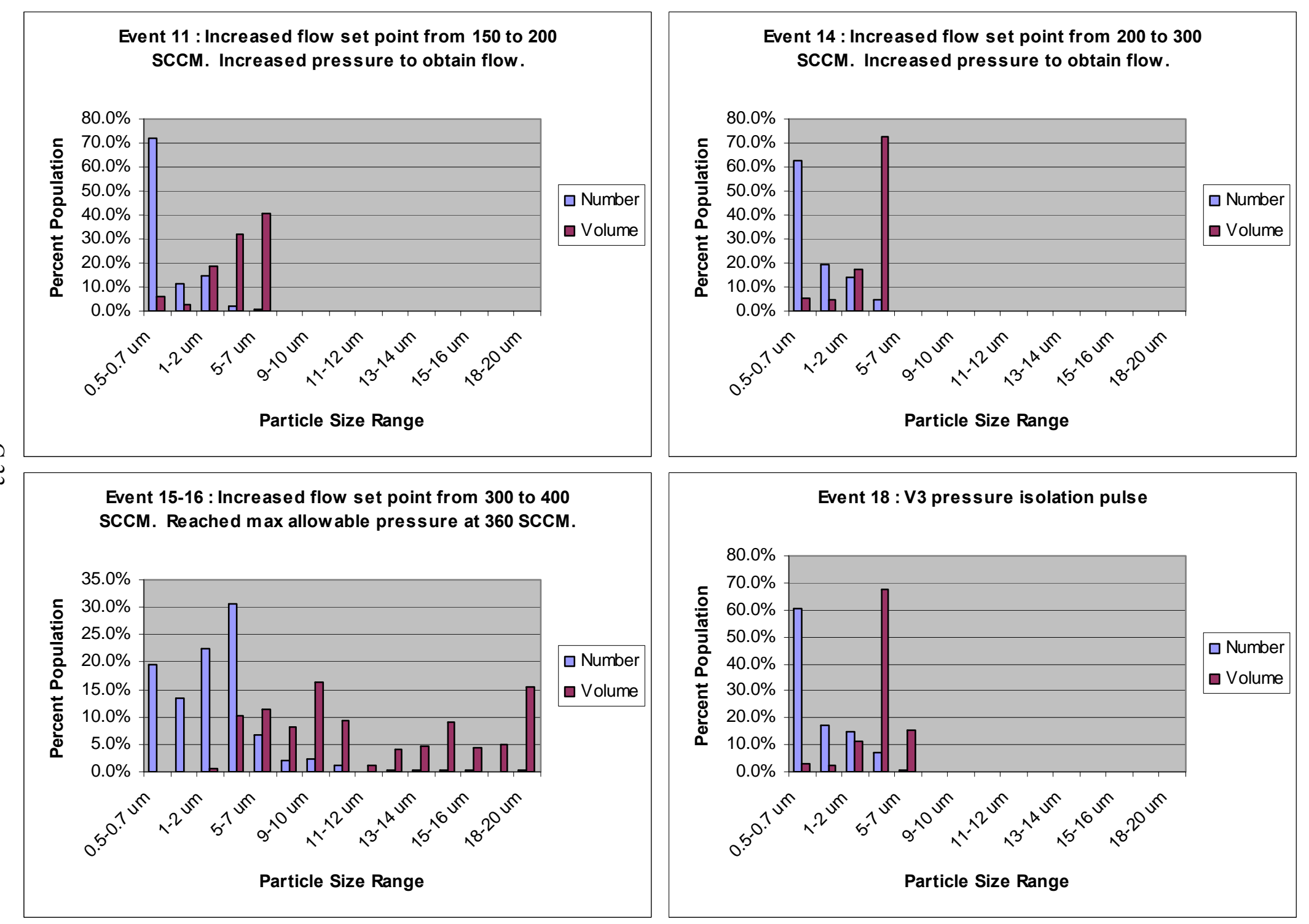

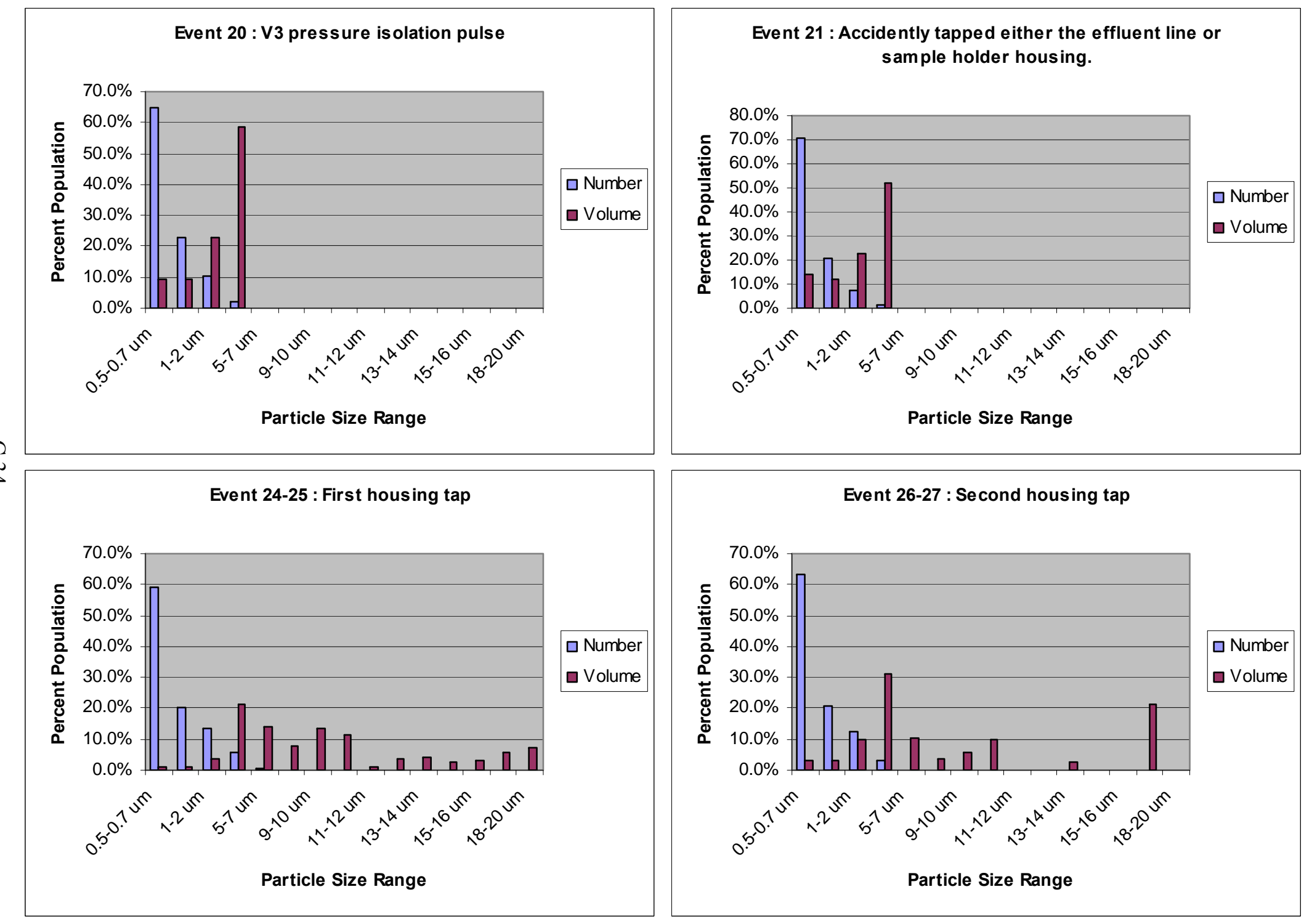
HBU-539B3Test 3

Event Total : Sum of all non-background release events

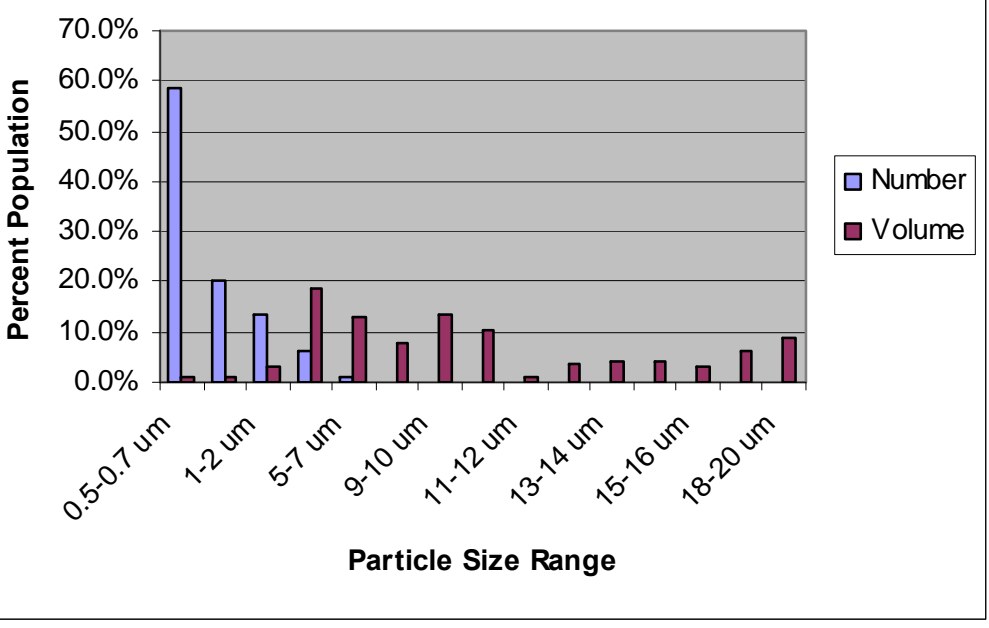

i 

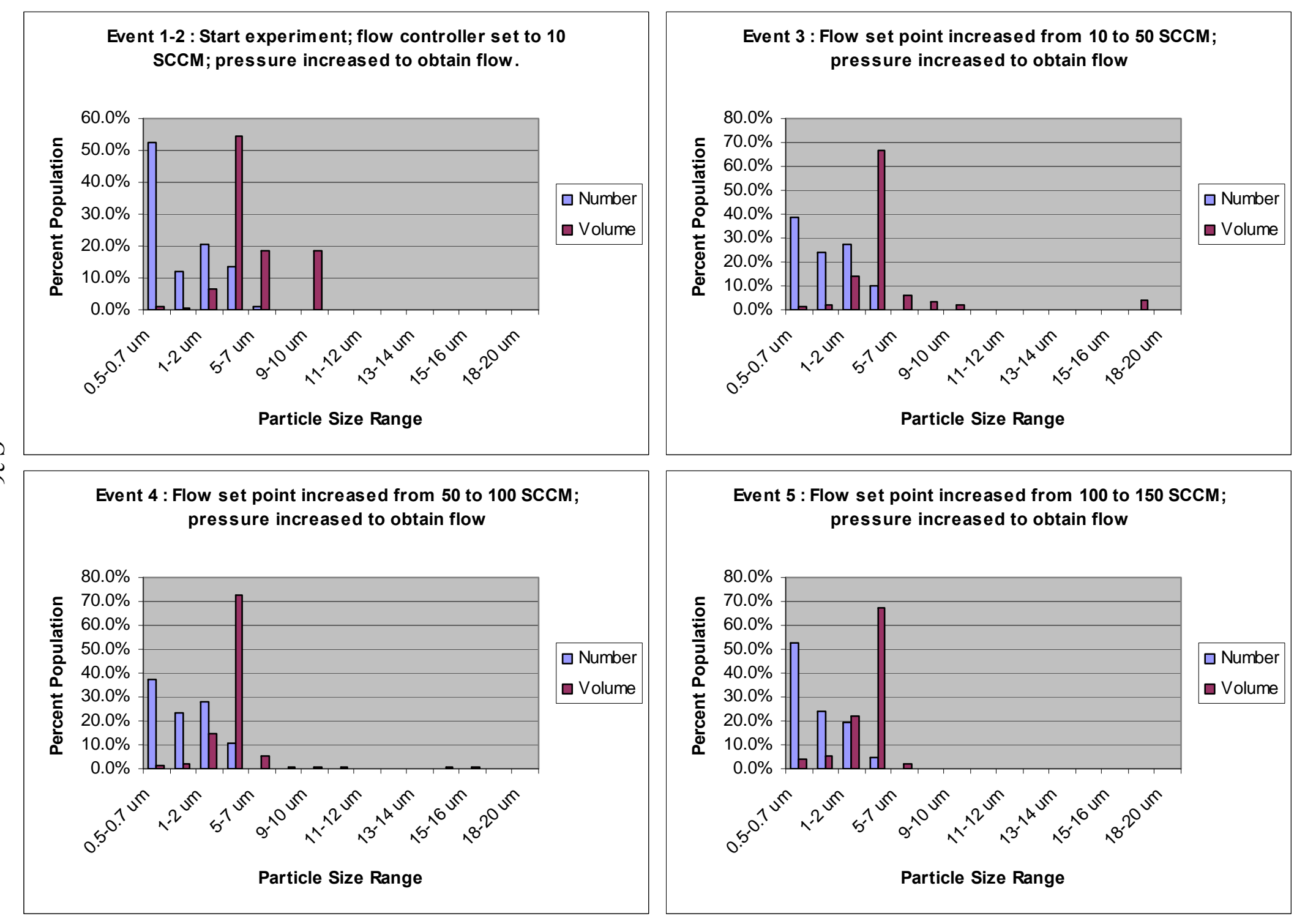

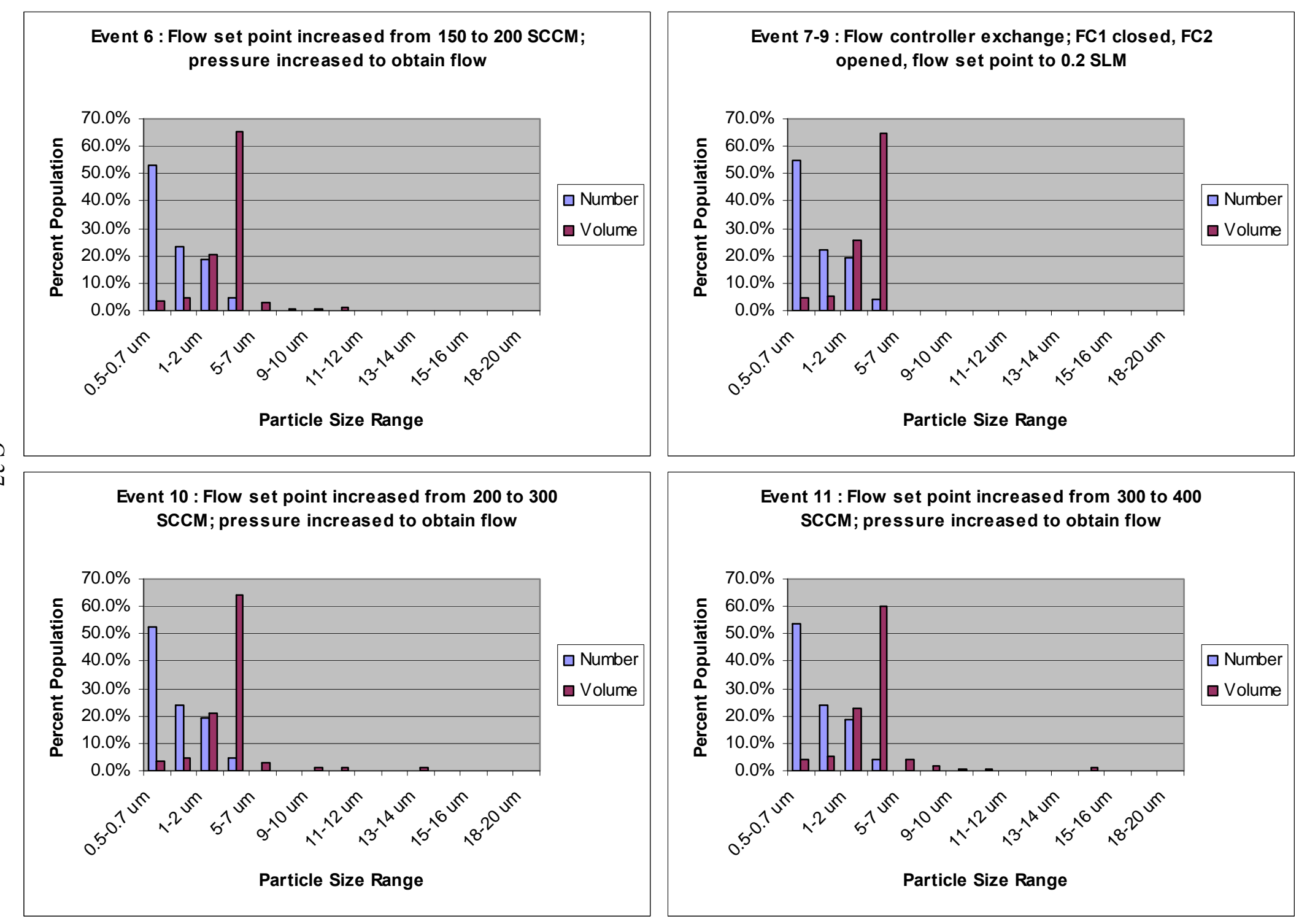

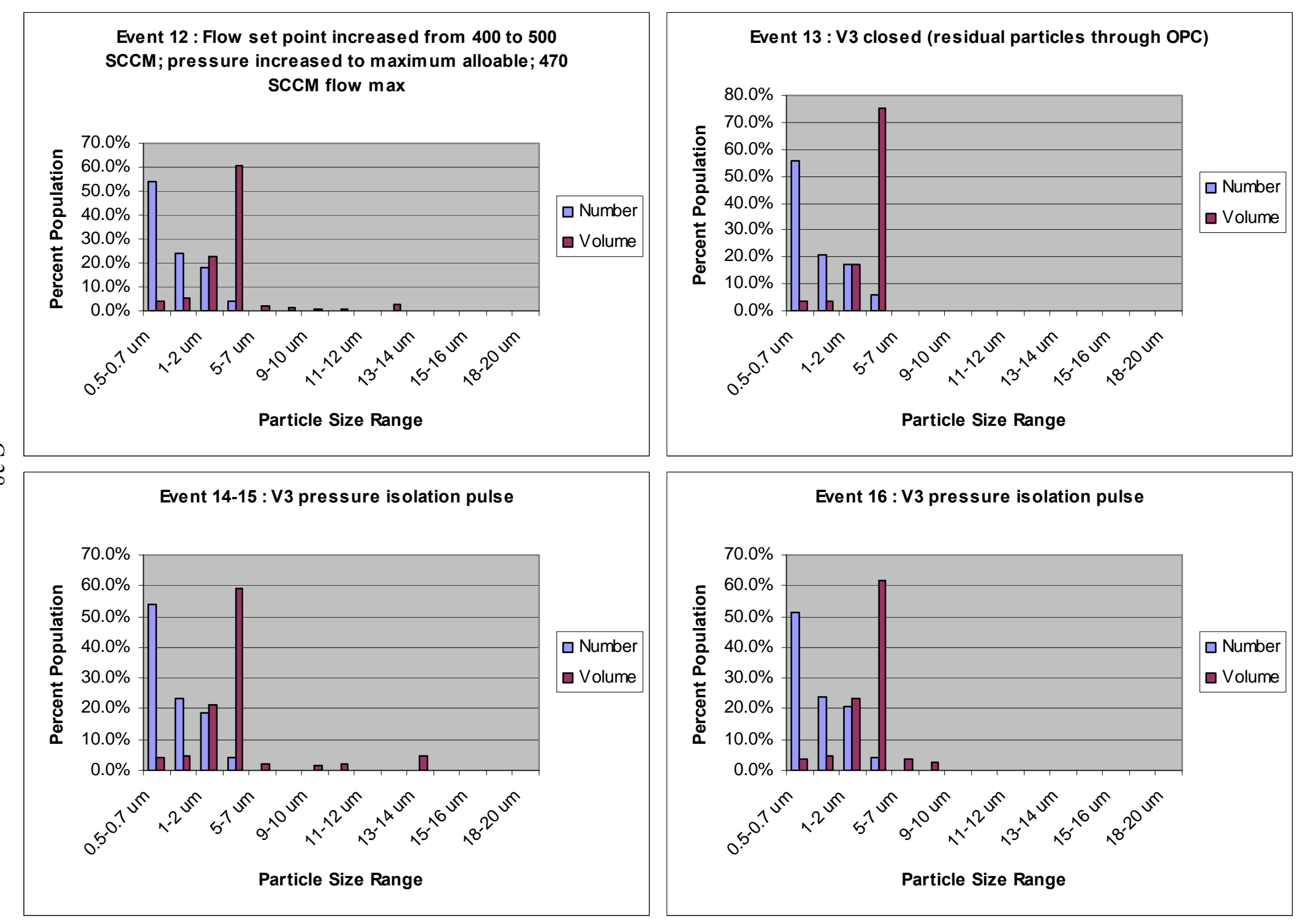

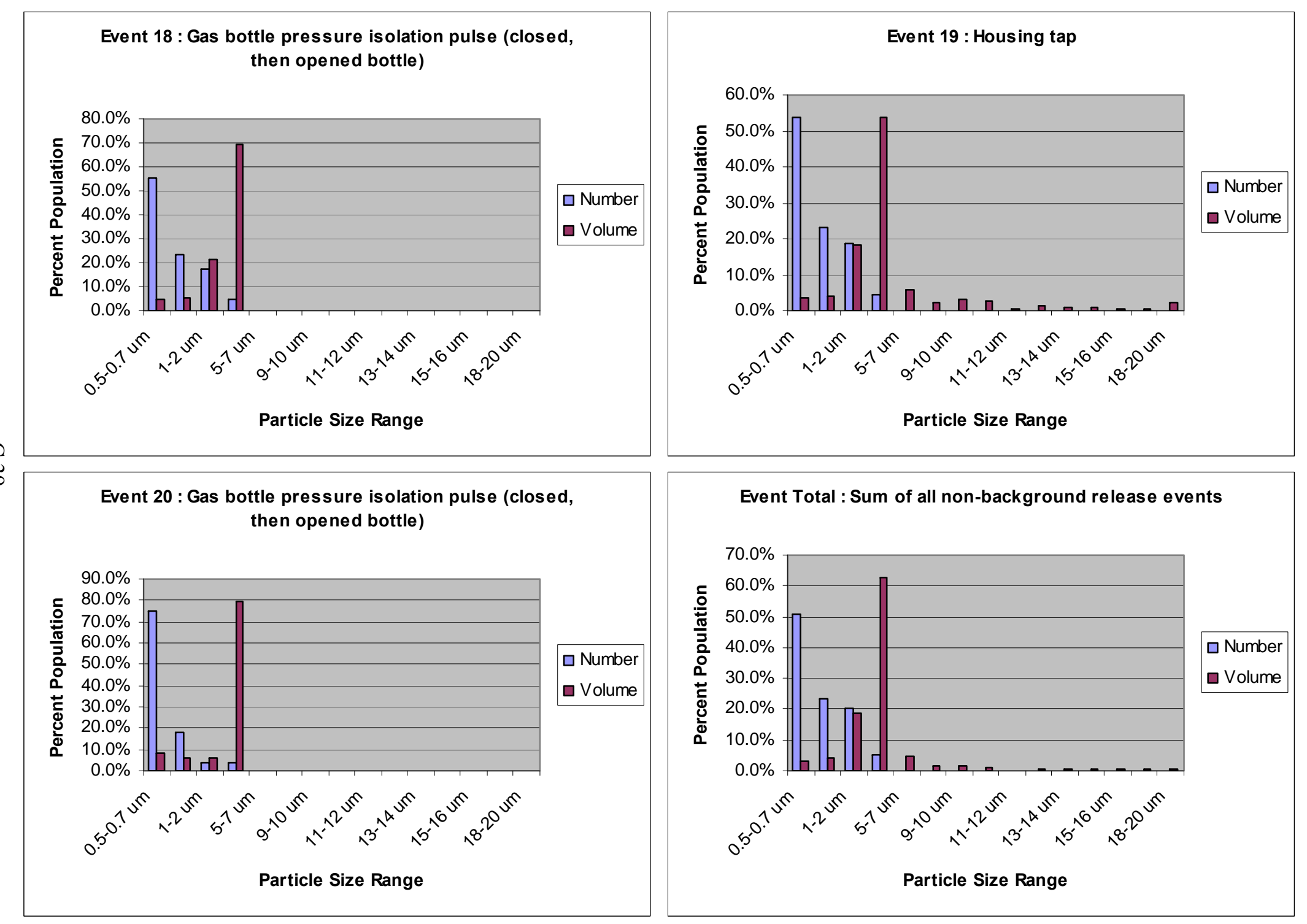

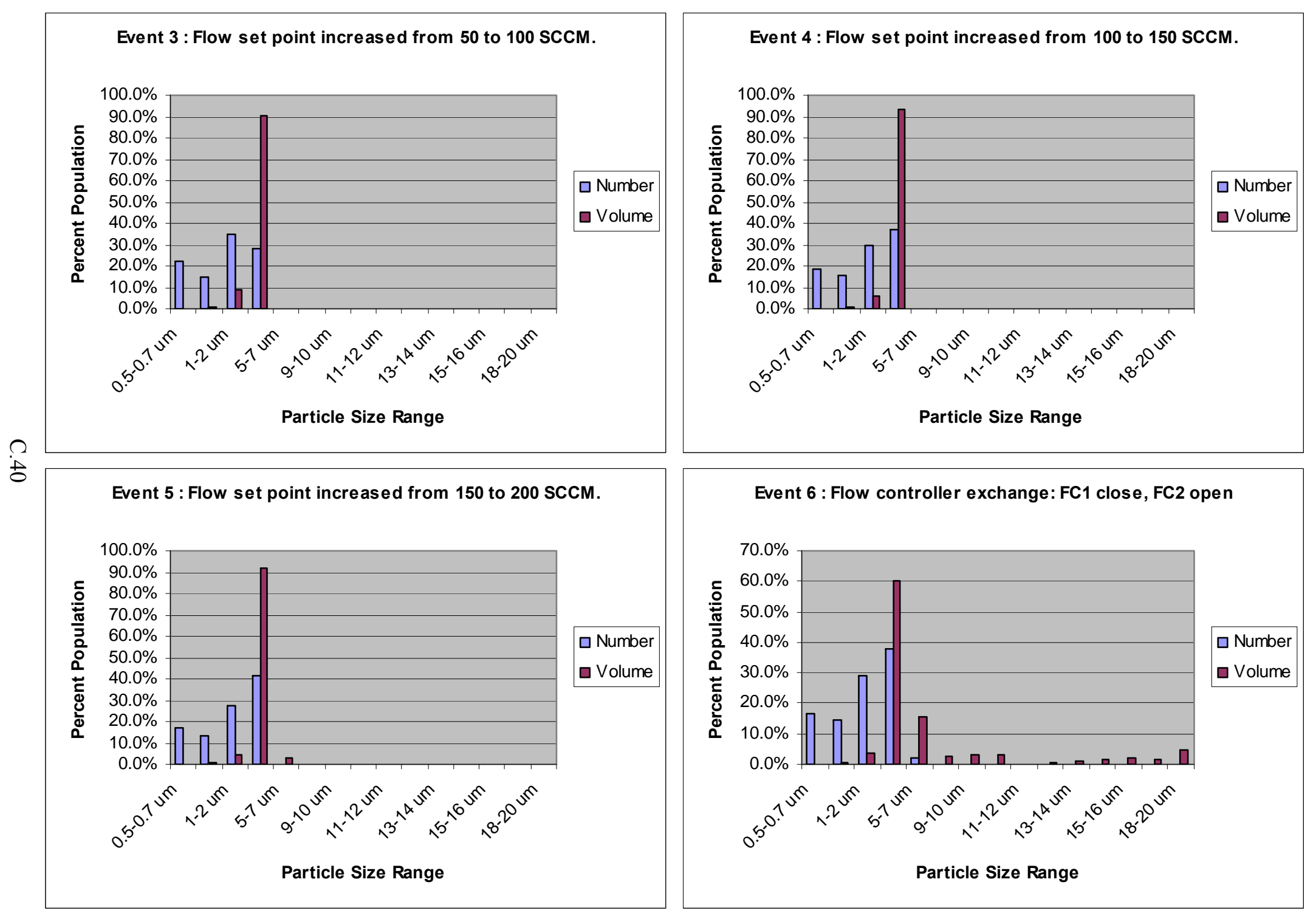

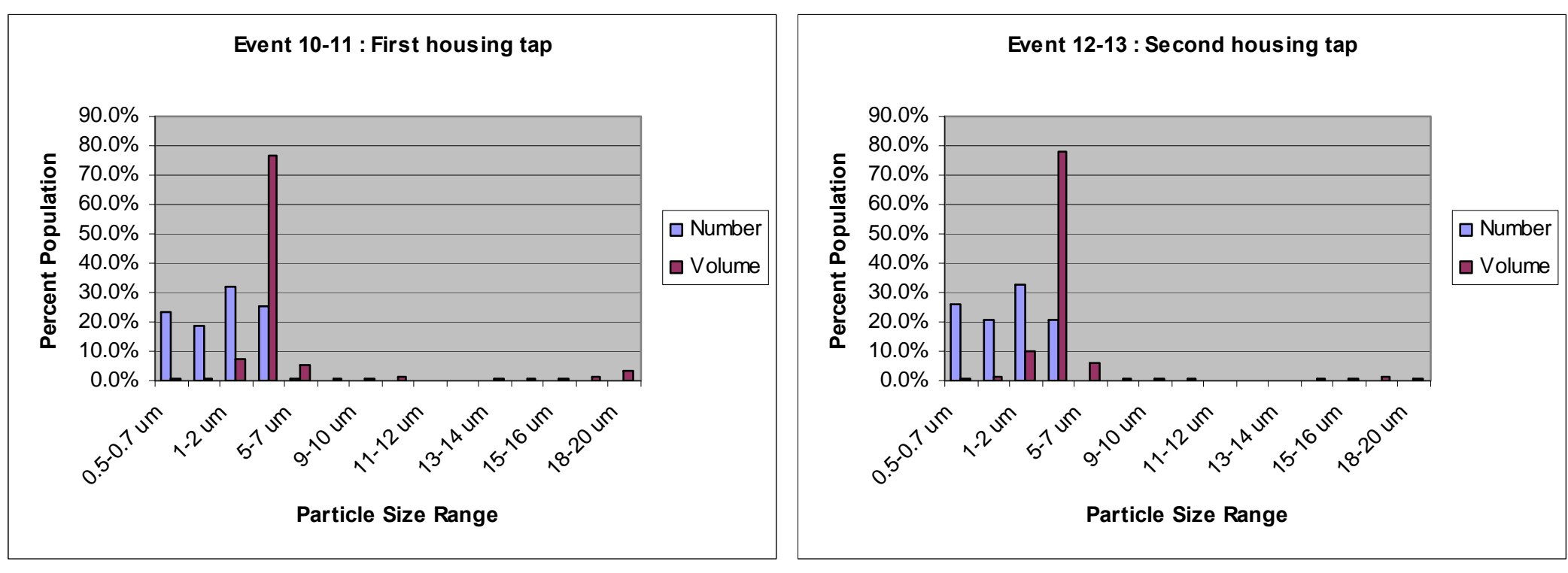

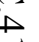

Event 14-15: Air inlet line to sample cham ber tapped.

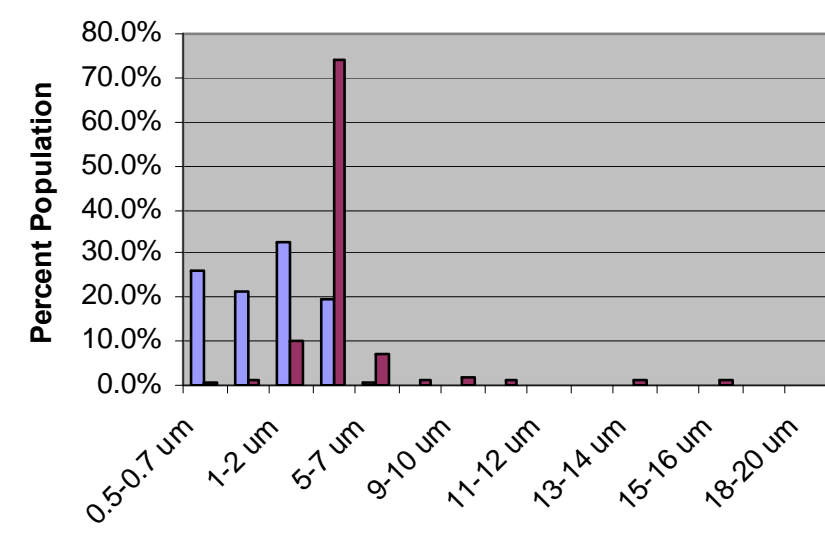

Particle Size Range

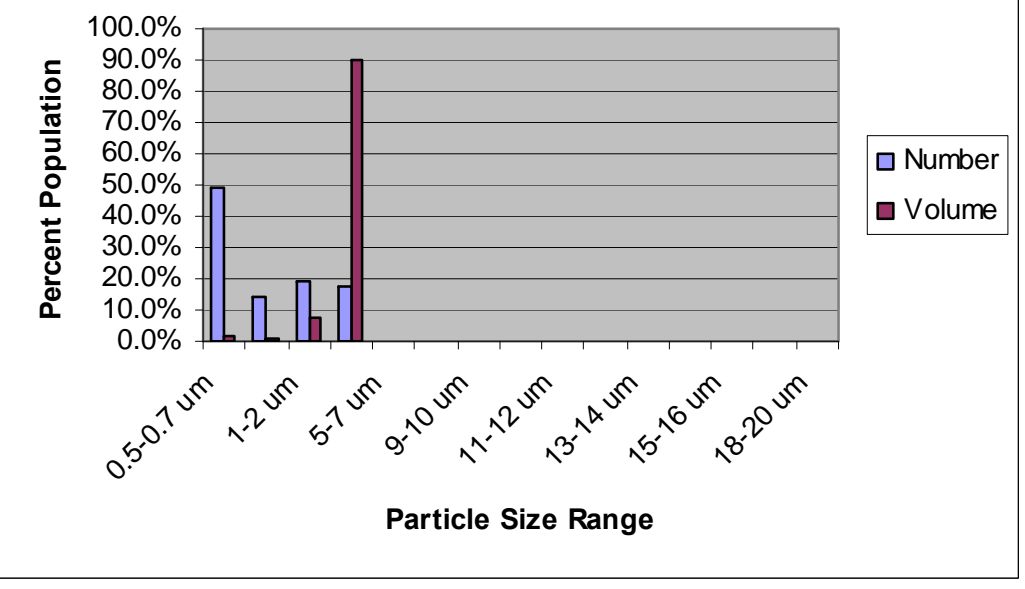



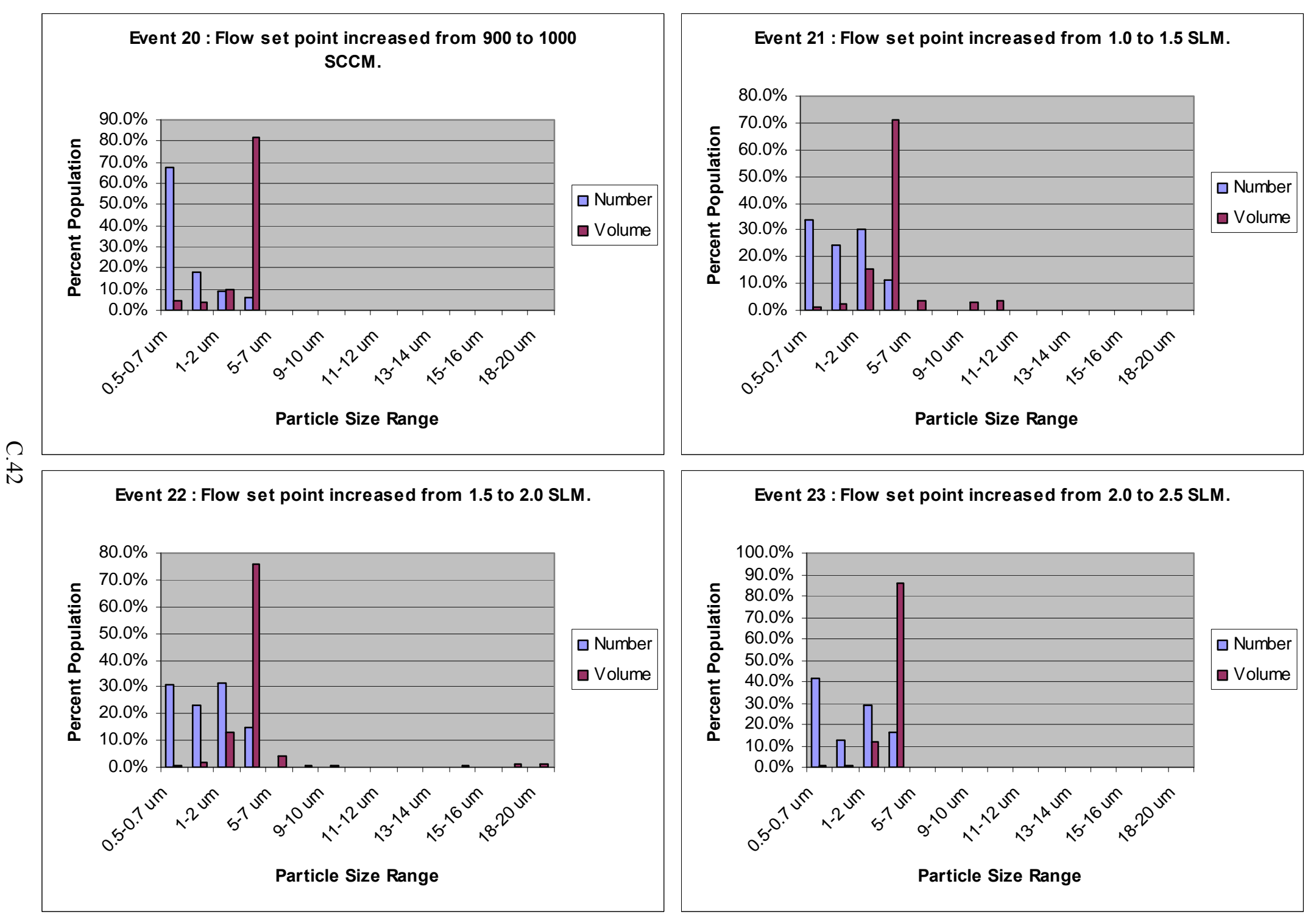

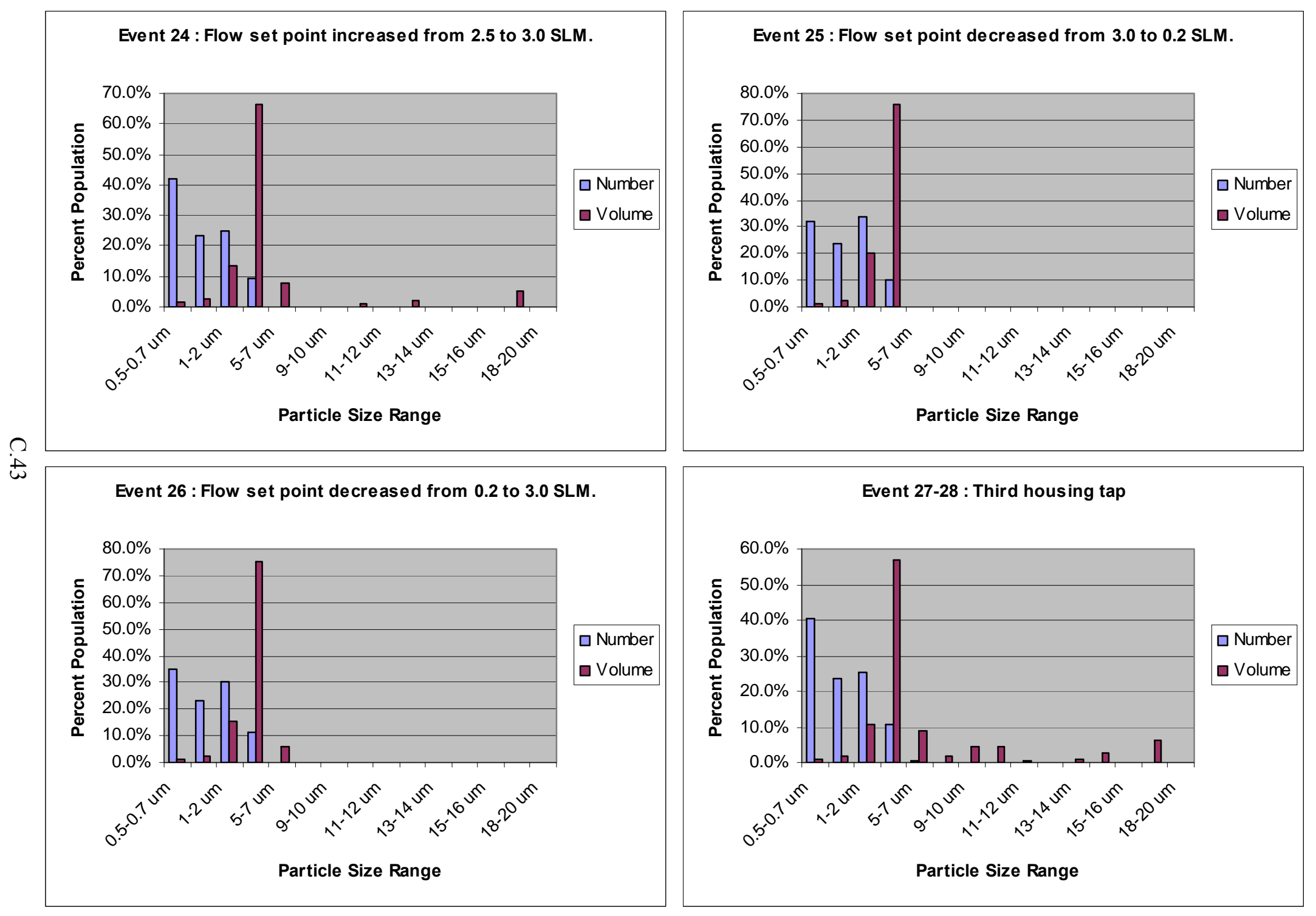

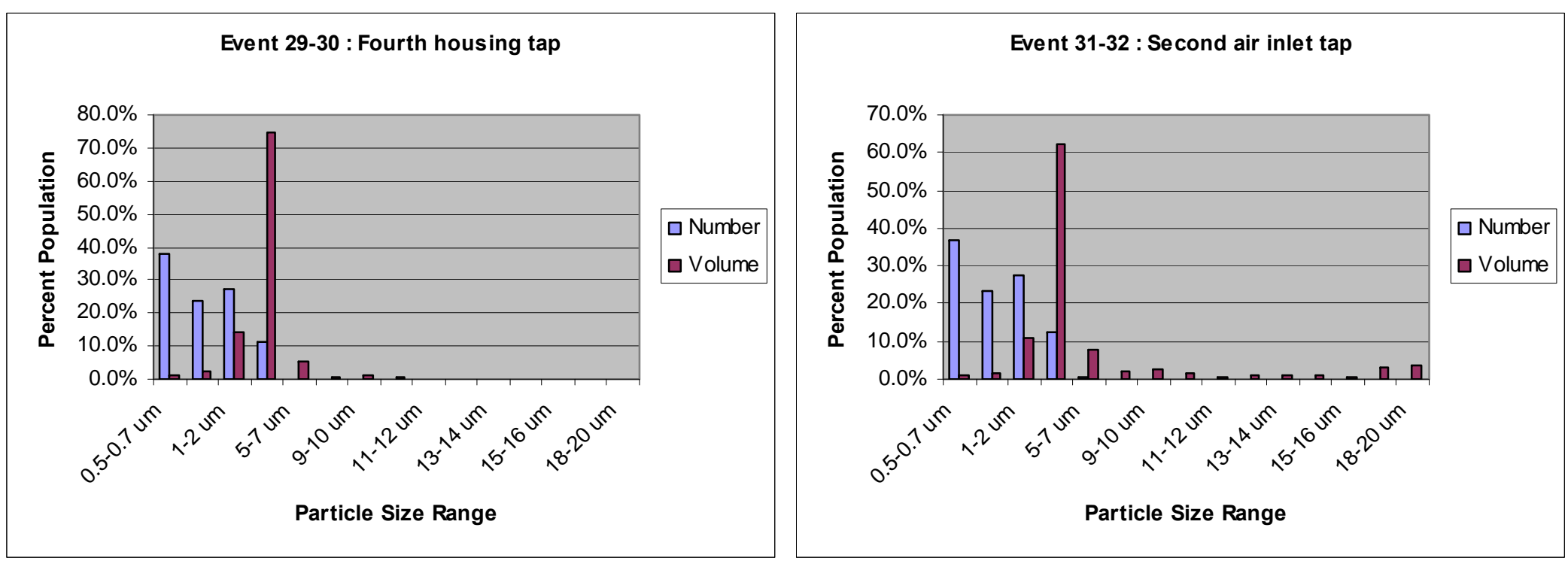

$\stackrel{A}{A}$
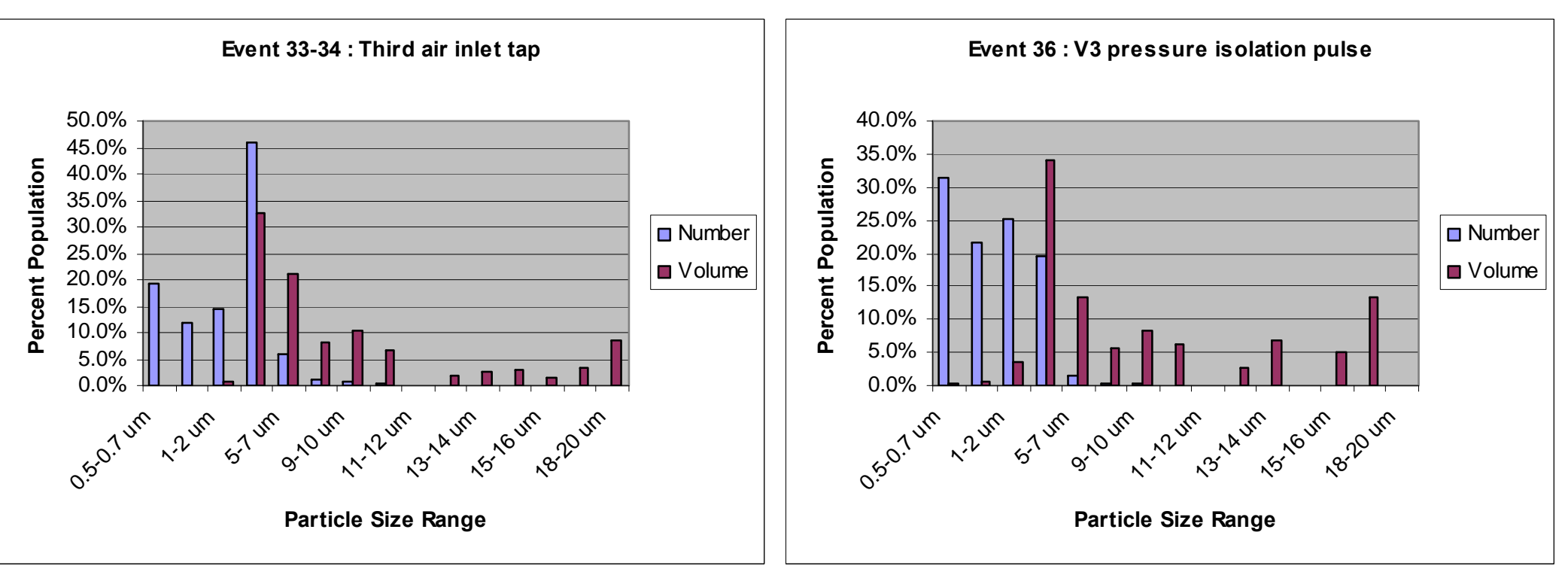

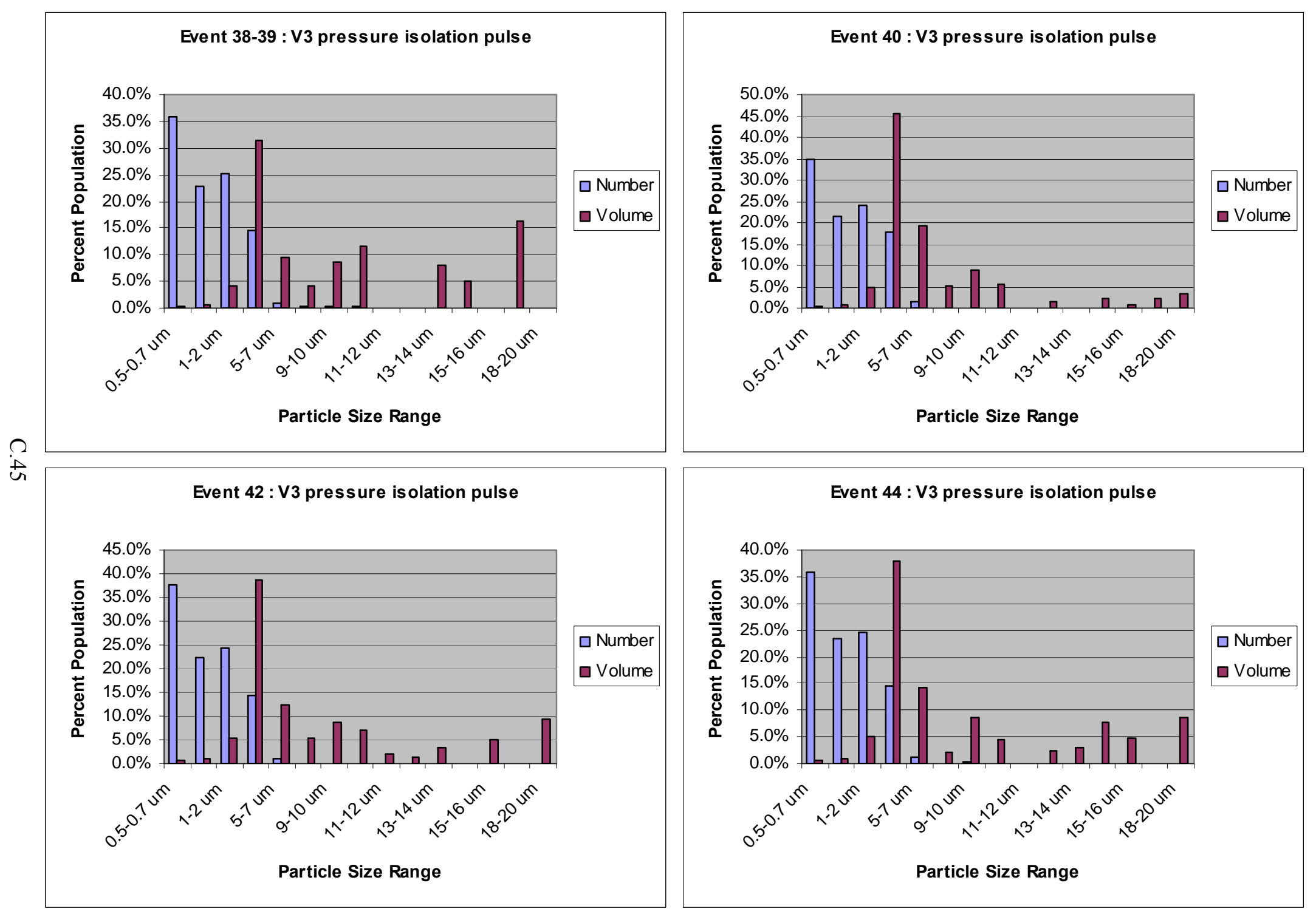

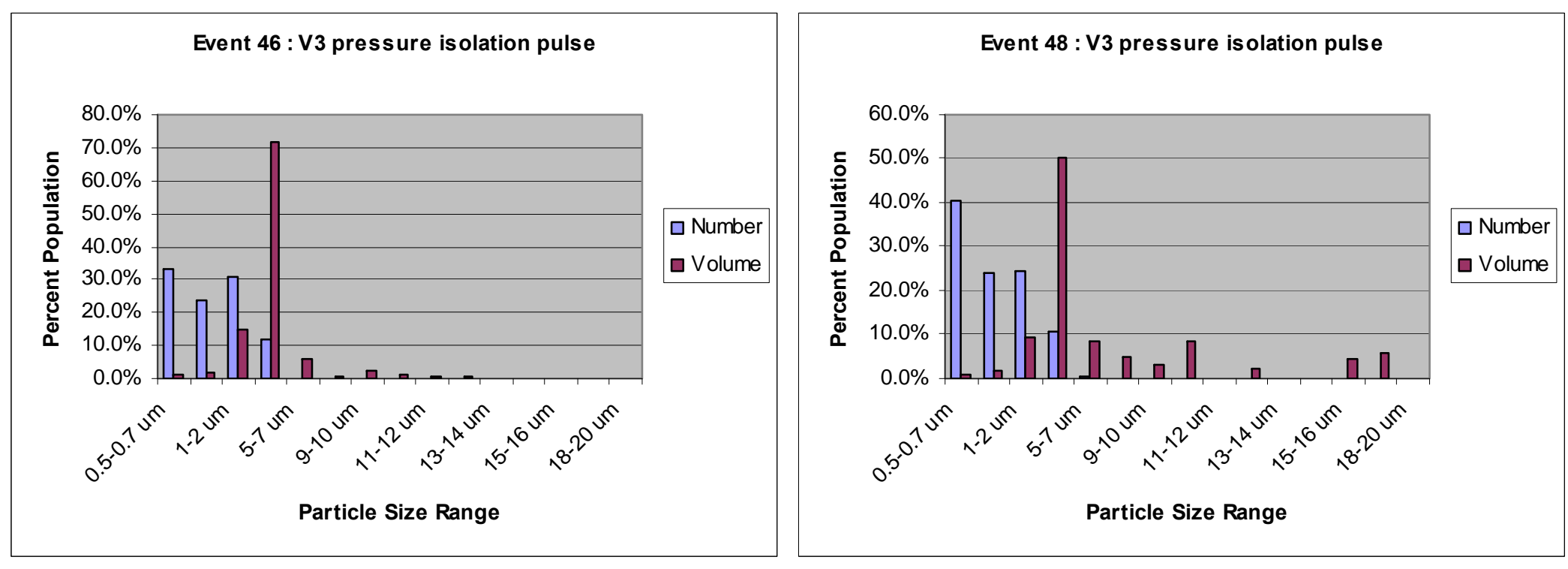

a
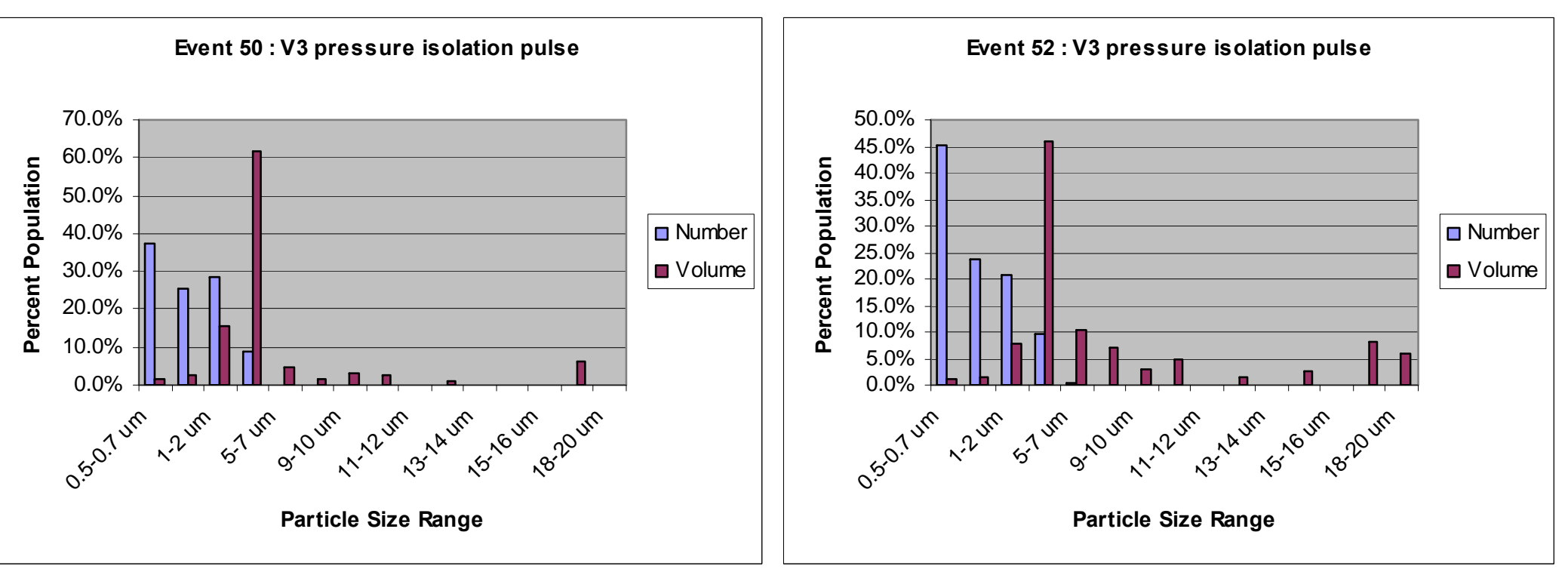

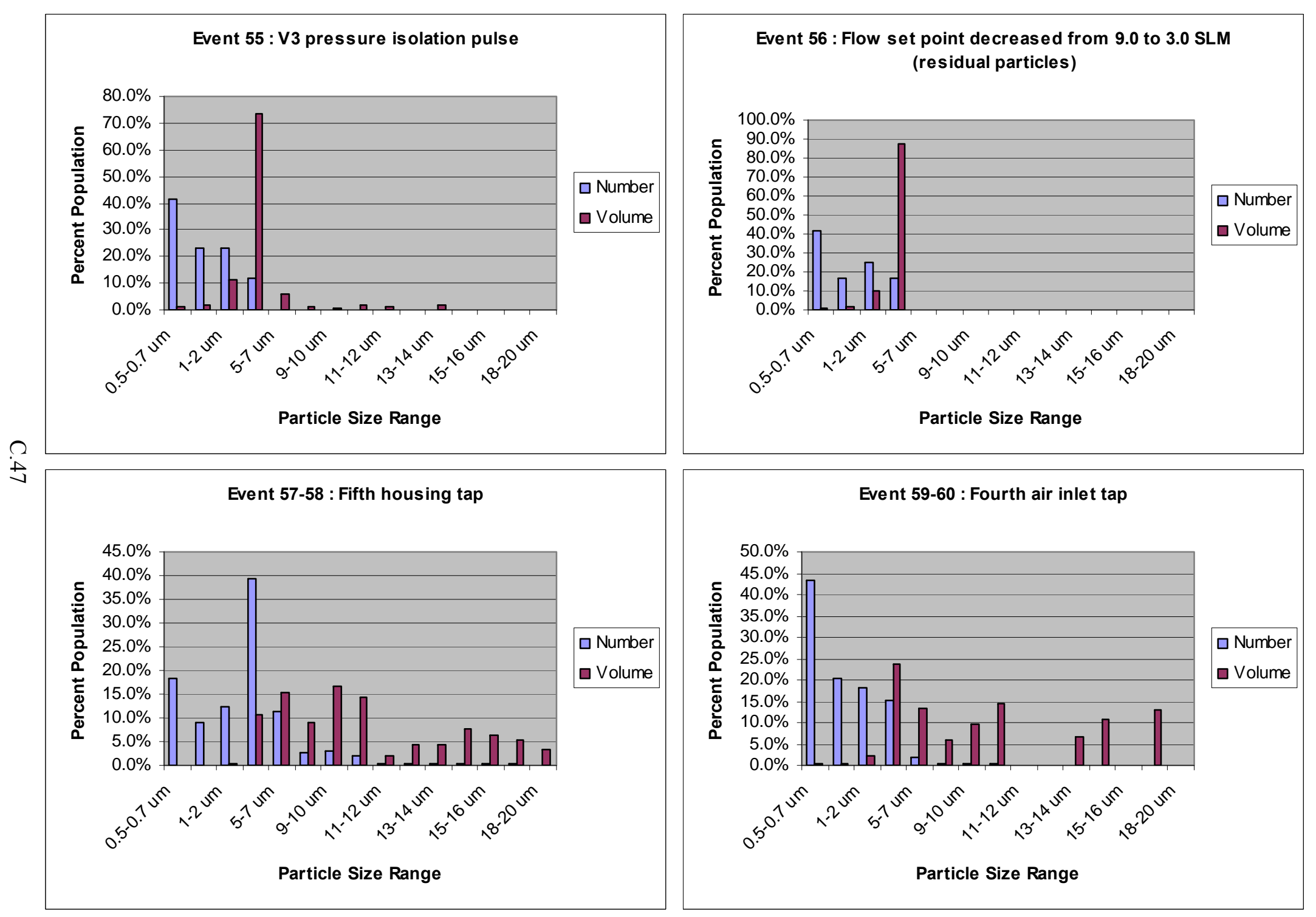
HBU-539F5 Test 1

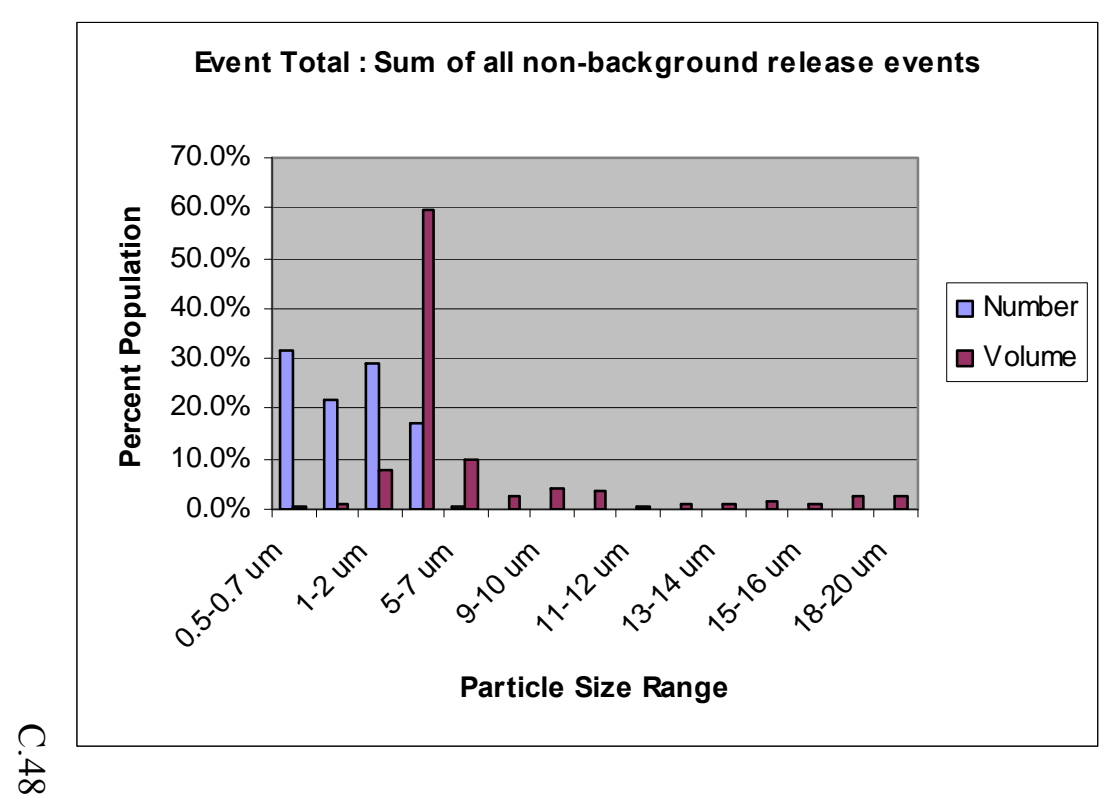



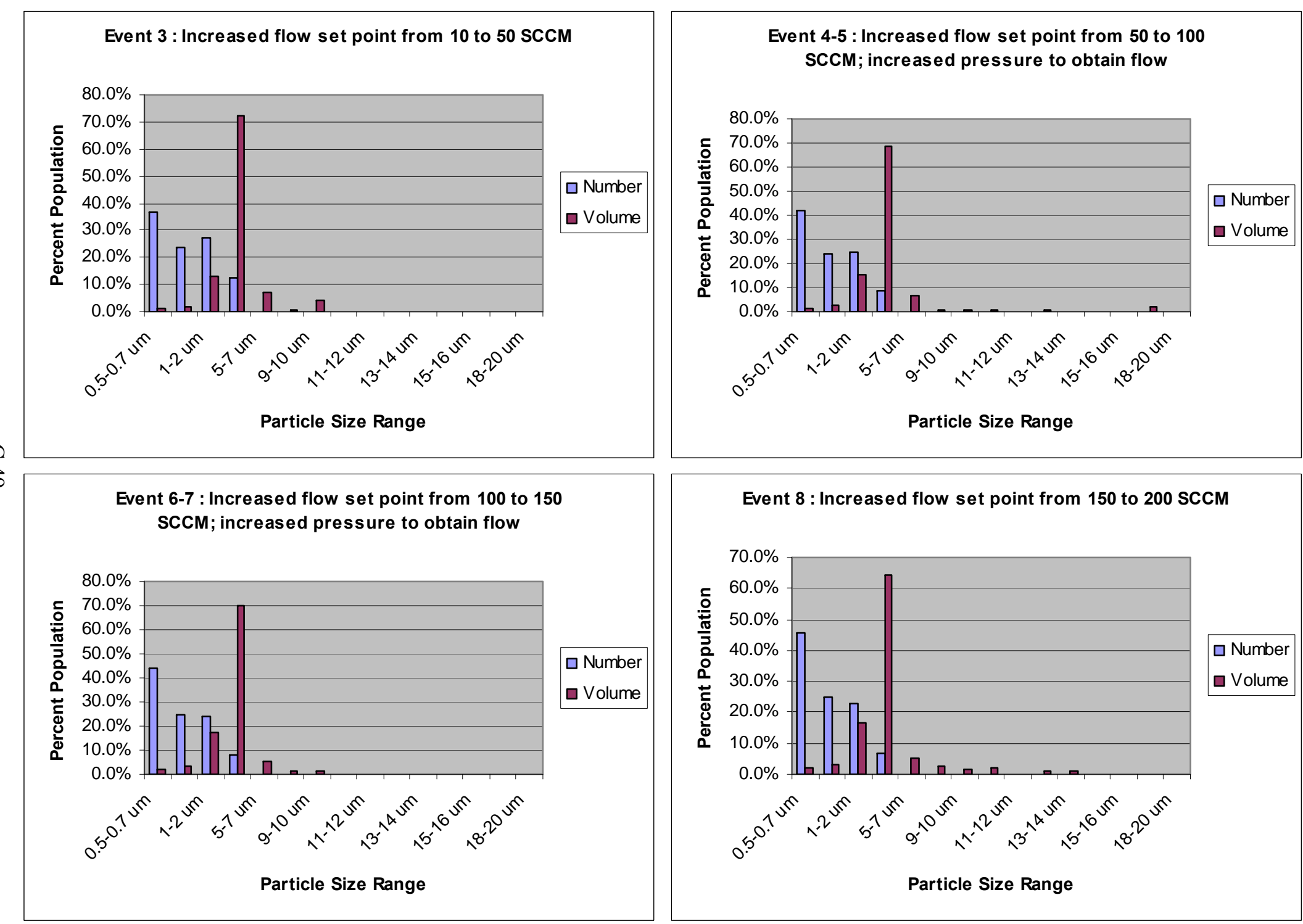

\section{Event 8 : Increased flow set point from 150 to 200 SCCM}

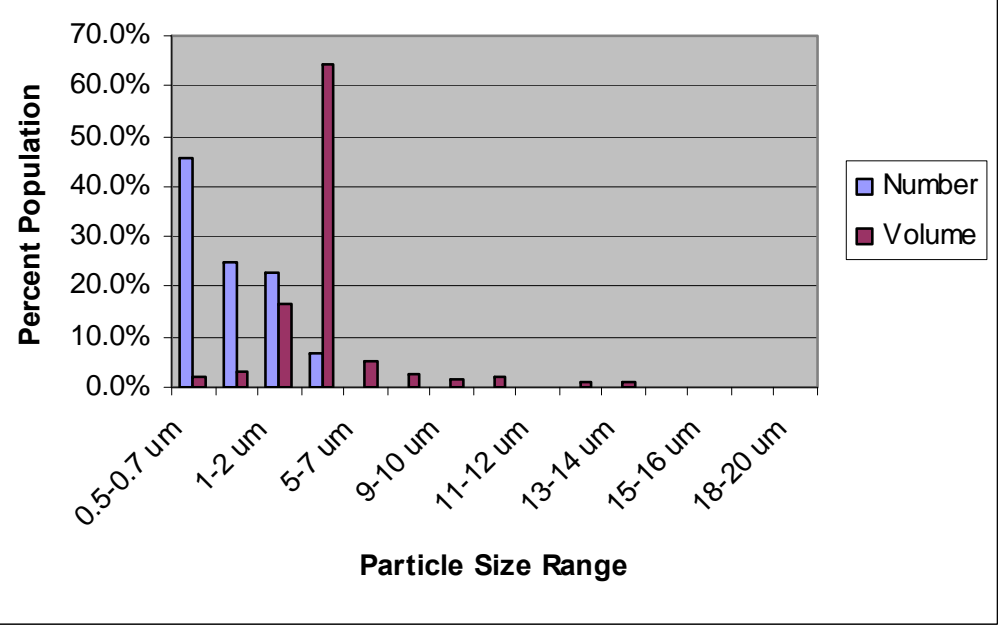


Event 9 : Flow controller exchange: FC1 closed, FC2 opened.
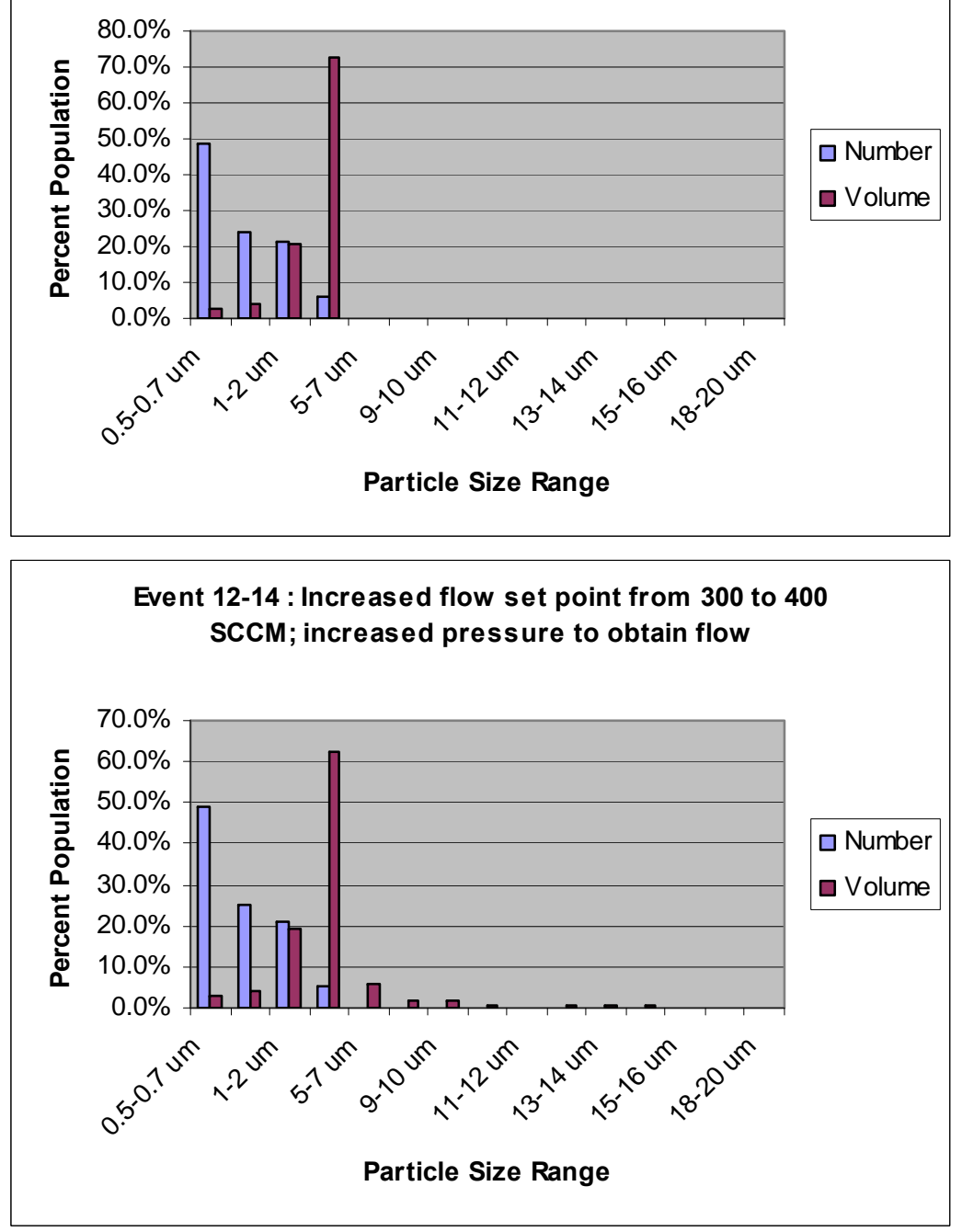

Event 10-11: Increased flow set point from 200 to 300 SCCM; increased pressure to obtain flow

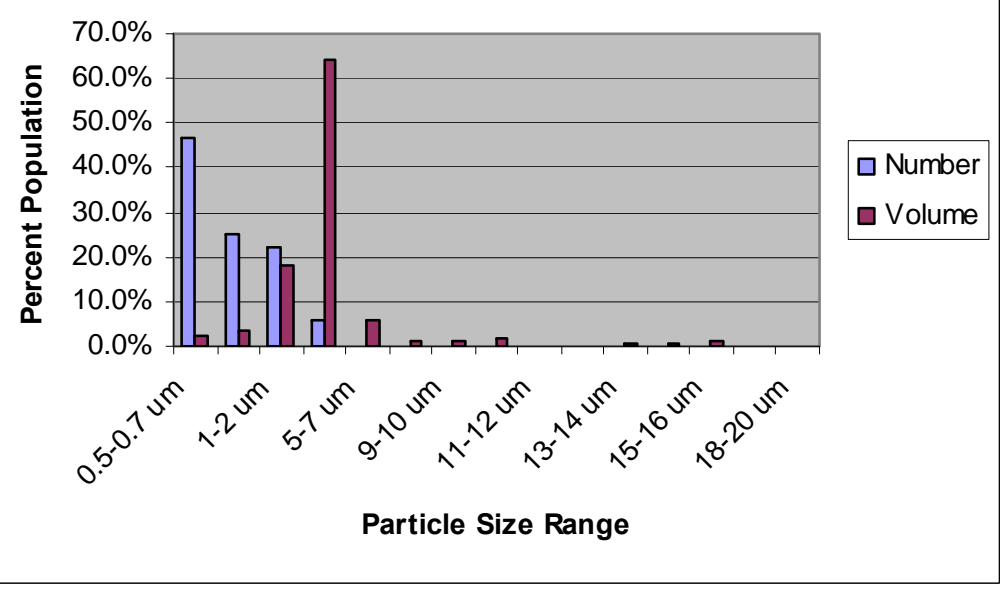

Event 15-16: Increased flow set point from 400 to 500 SCCM; increased pressure to obtain flow

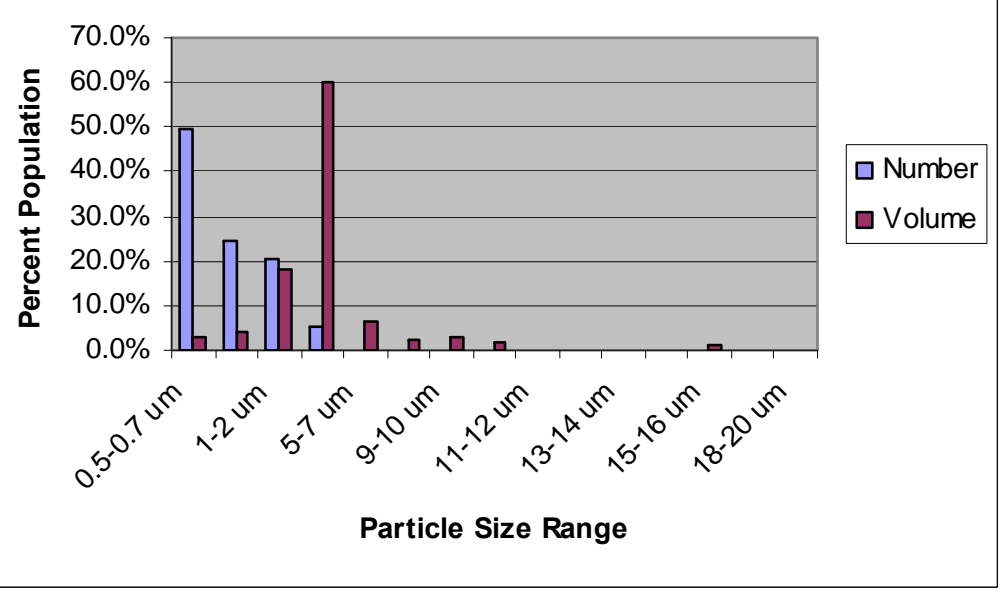



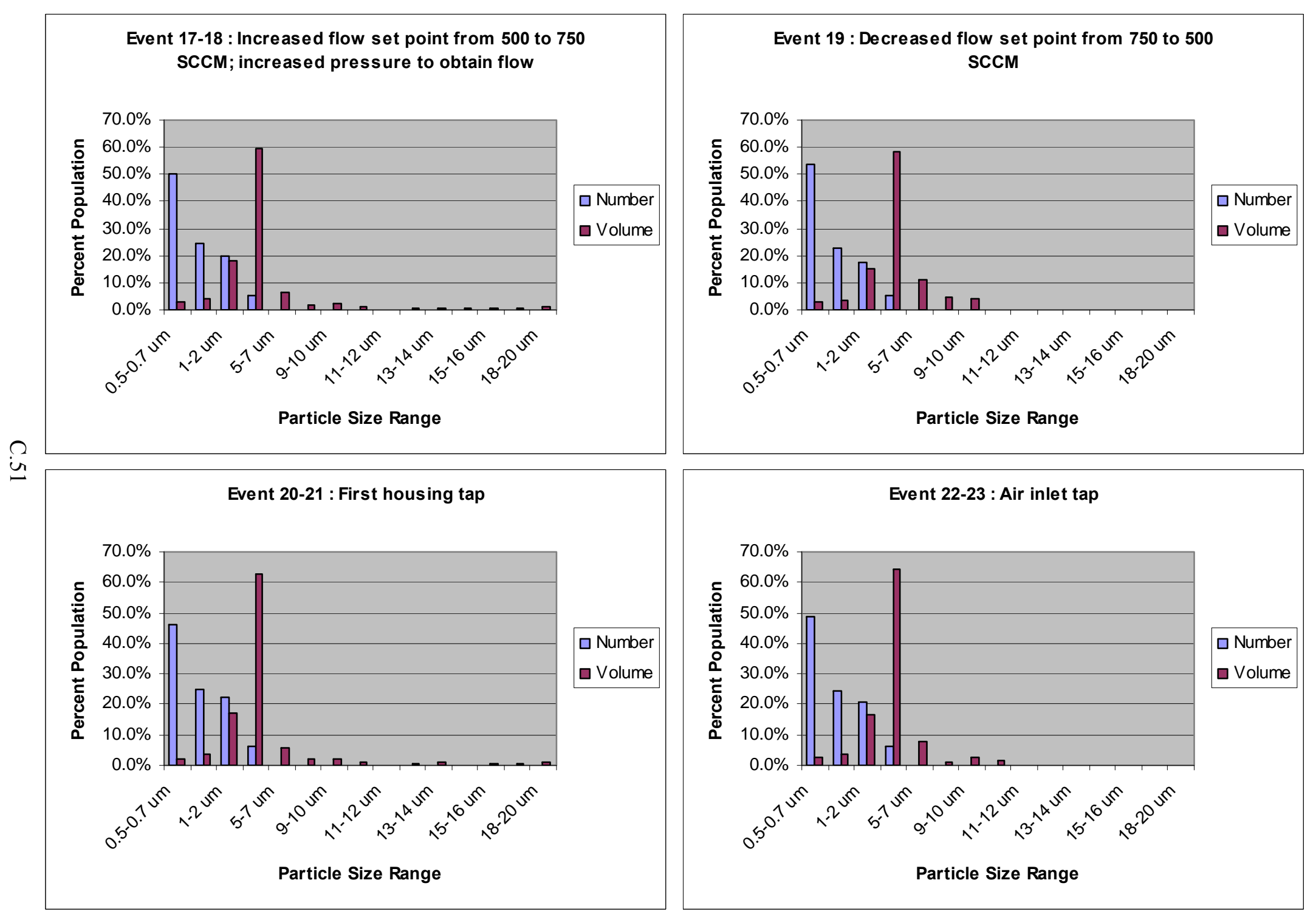

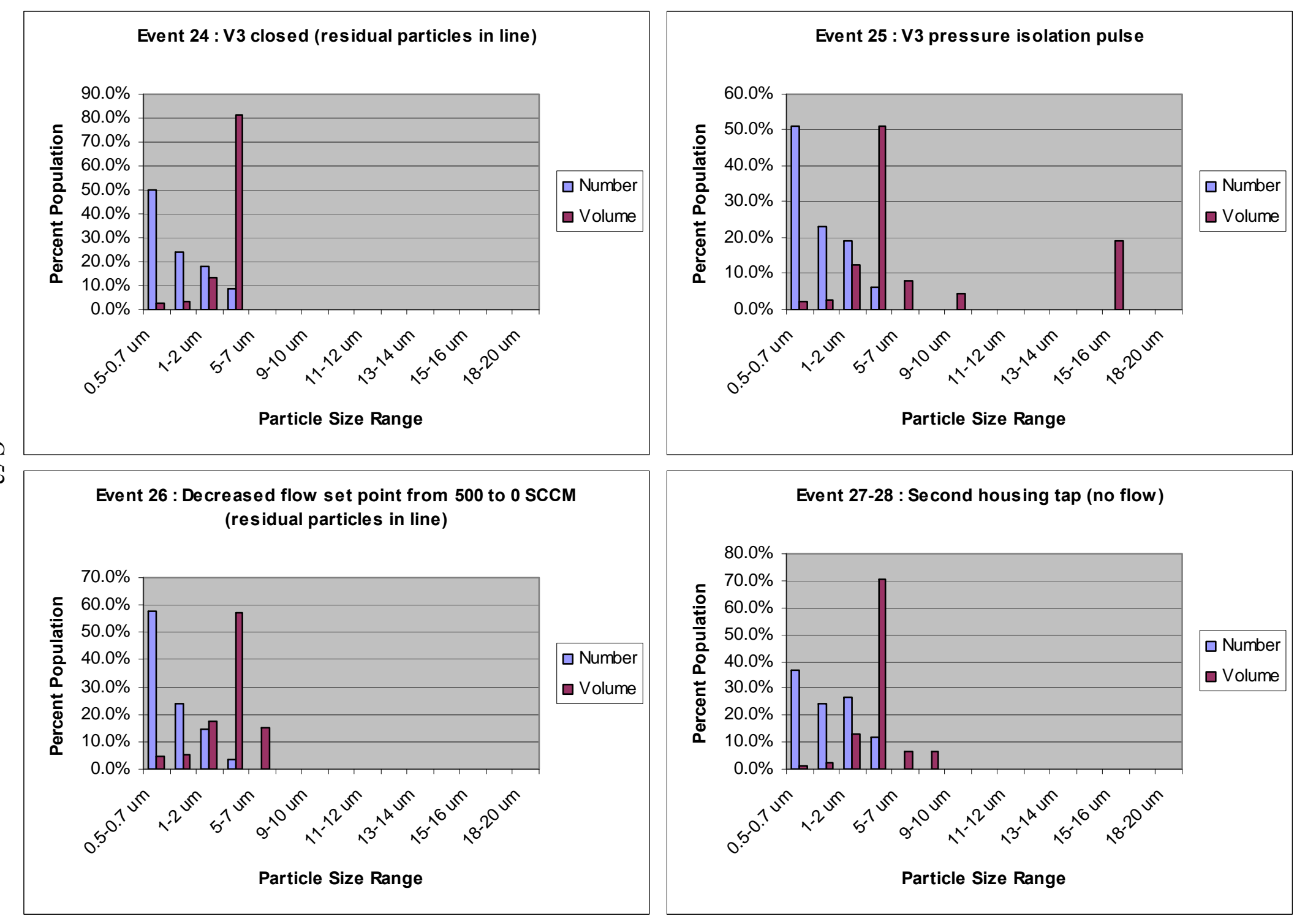
HBU-539F5 Test 2
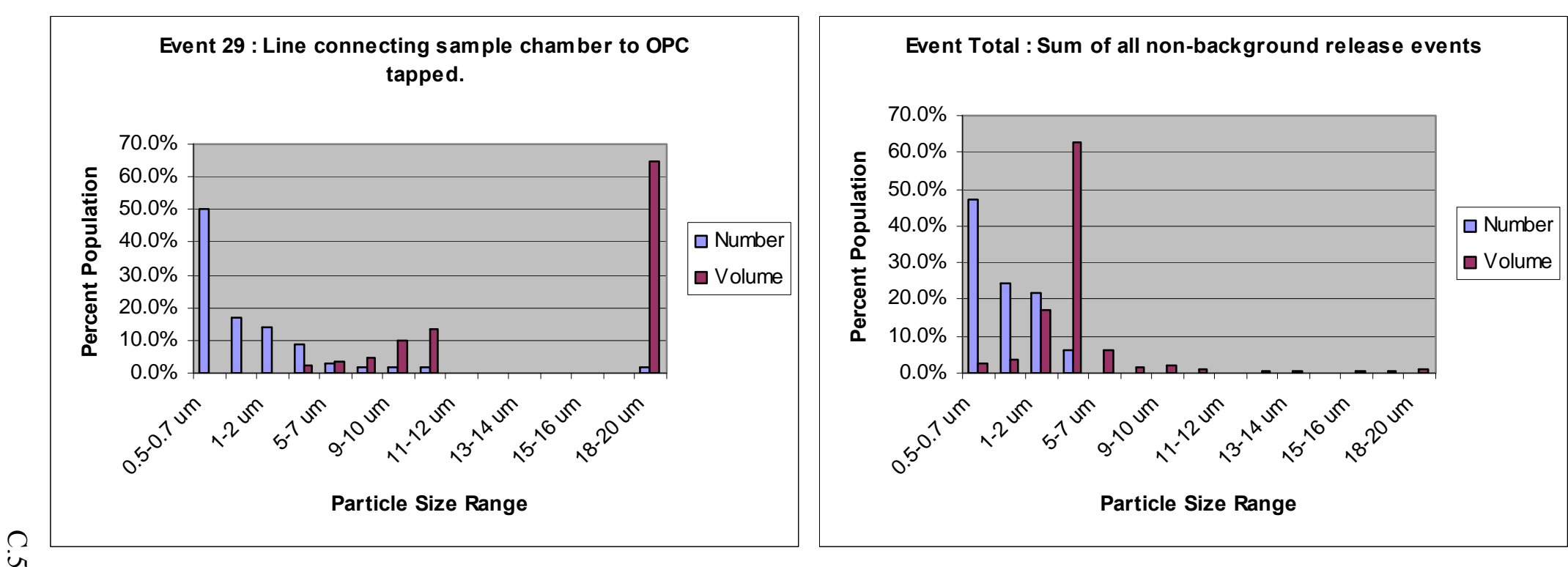



\section{Appendix D}

\section{Particle-Size Distributions from CSNF Powder Tests}

This appendix contains a histogram of the particle-size distribution for each significant event of each CSNF powder test. Each size bin in the histogram presents the volume fraction and the number fraction of the total particle population belonging to the bin. 


\section{Appendix D: Particle-Size Distributions for CSNF Powder Tests}
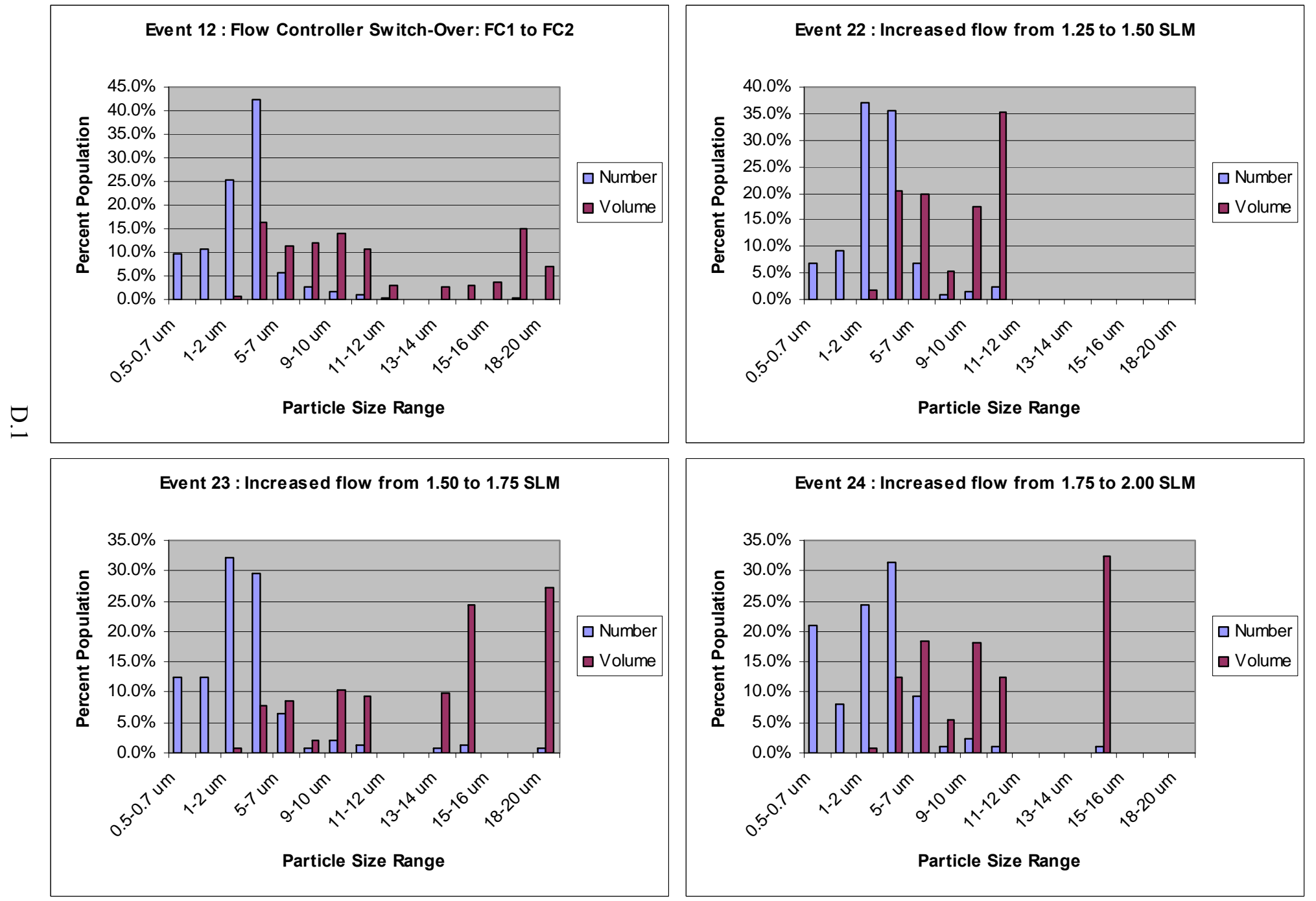

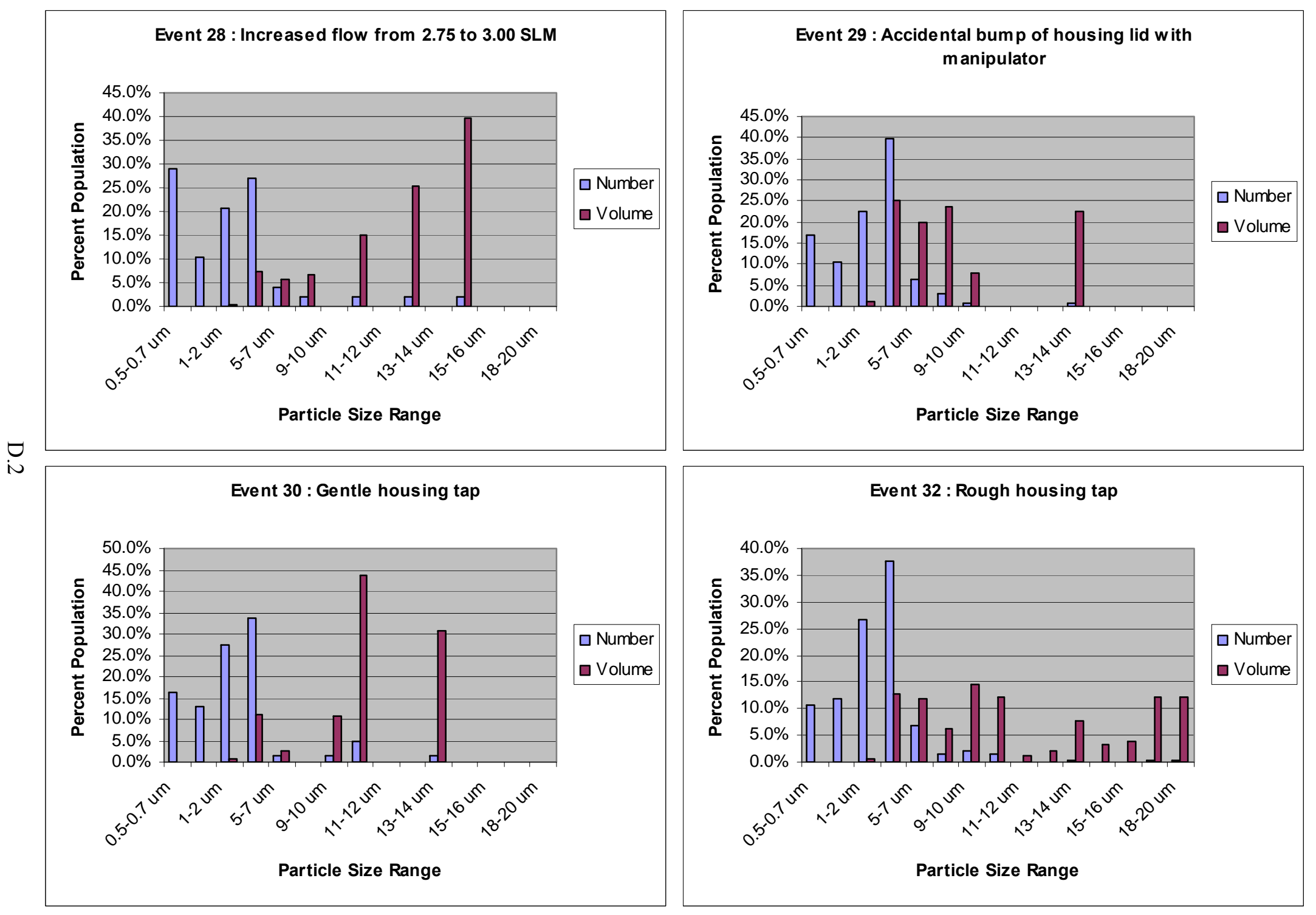

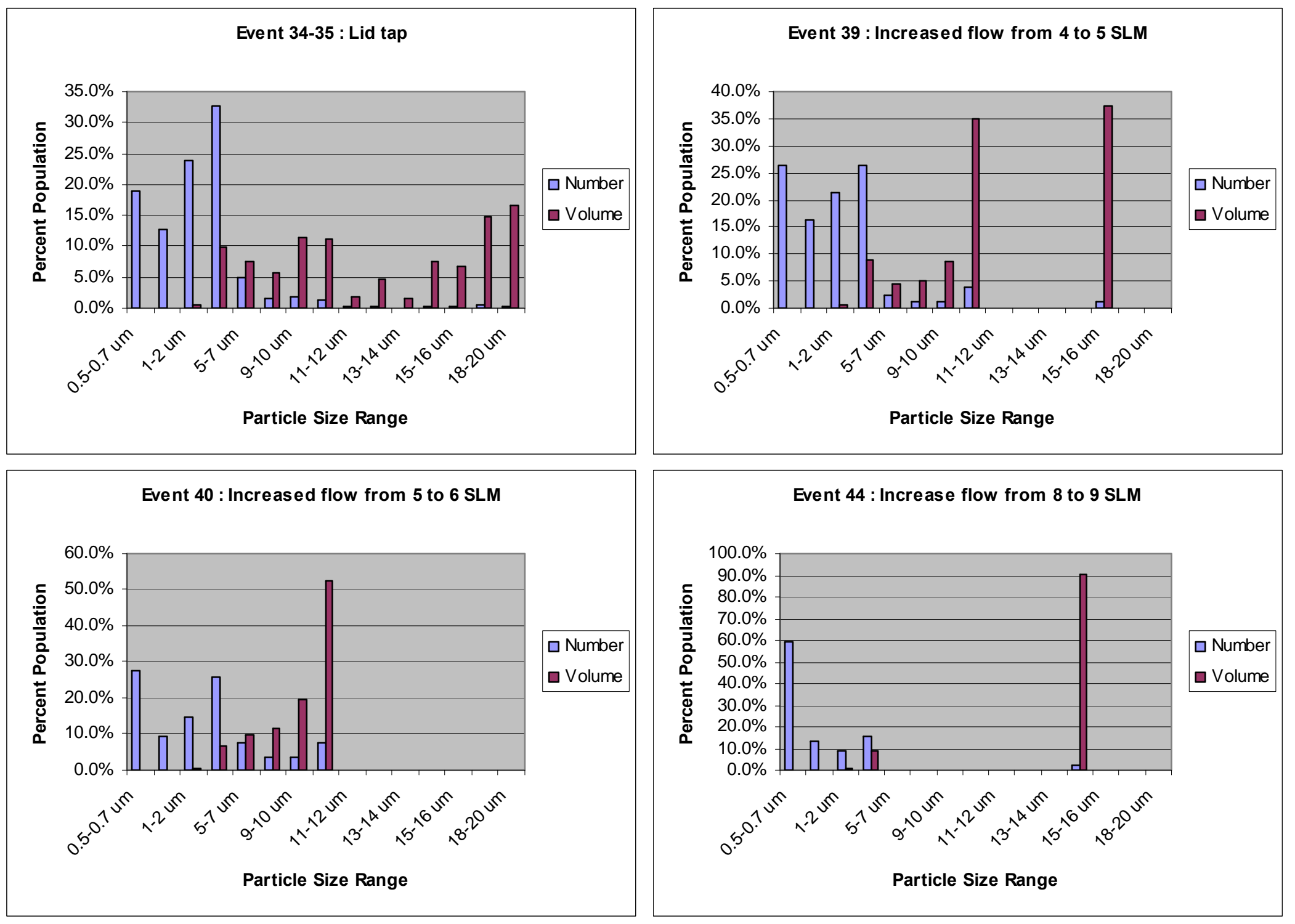

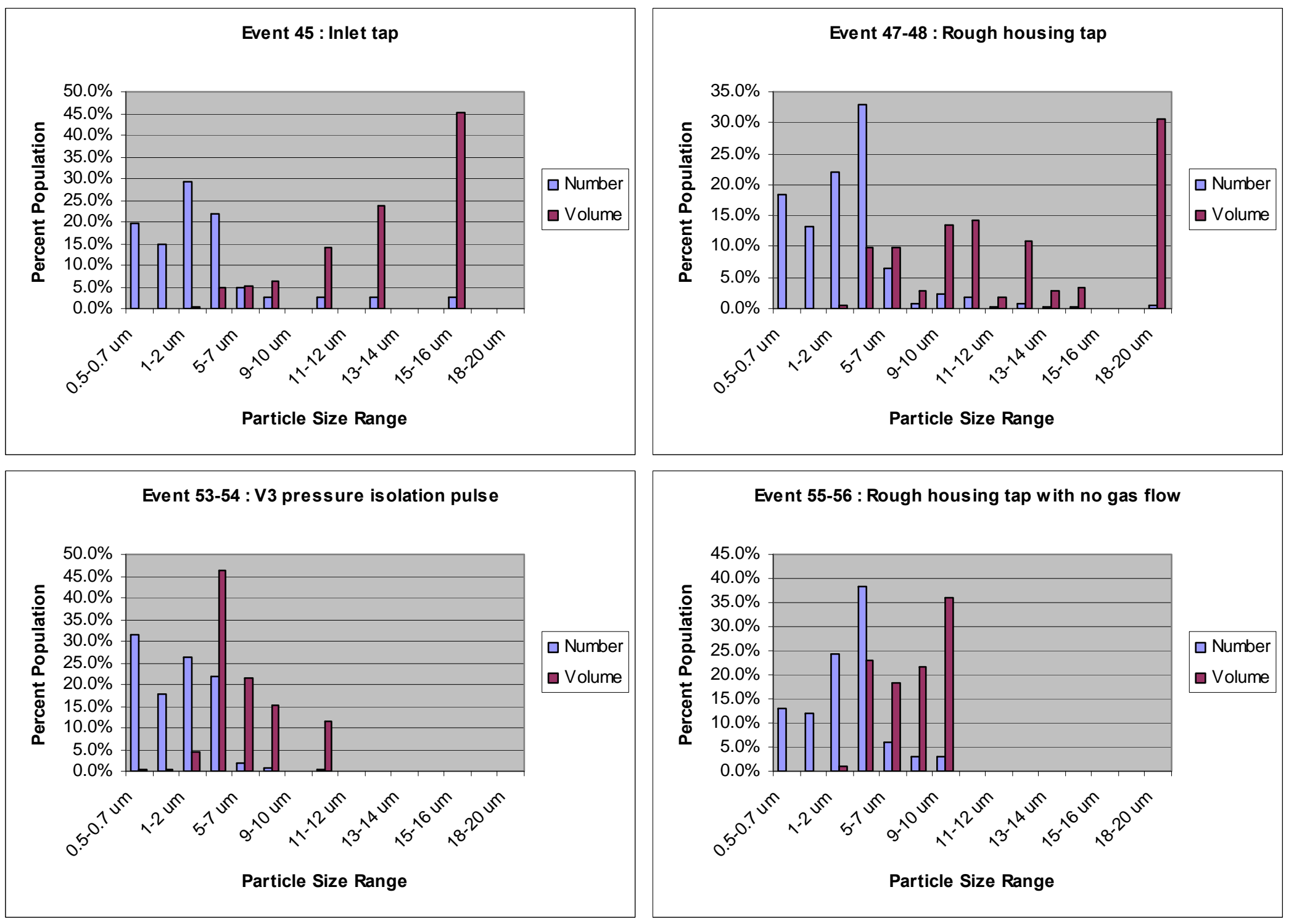

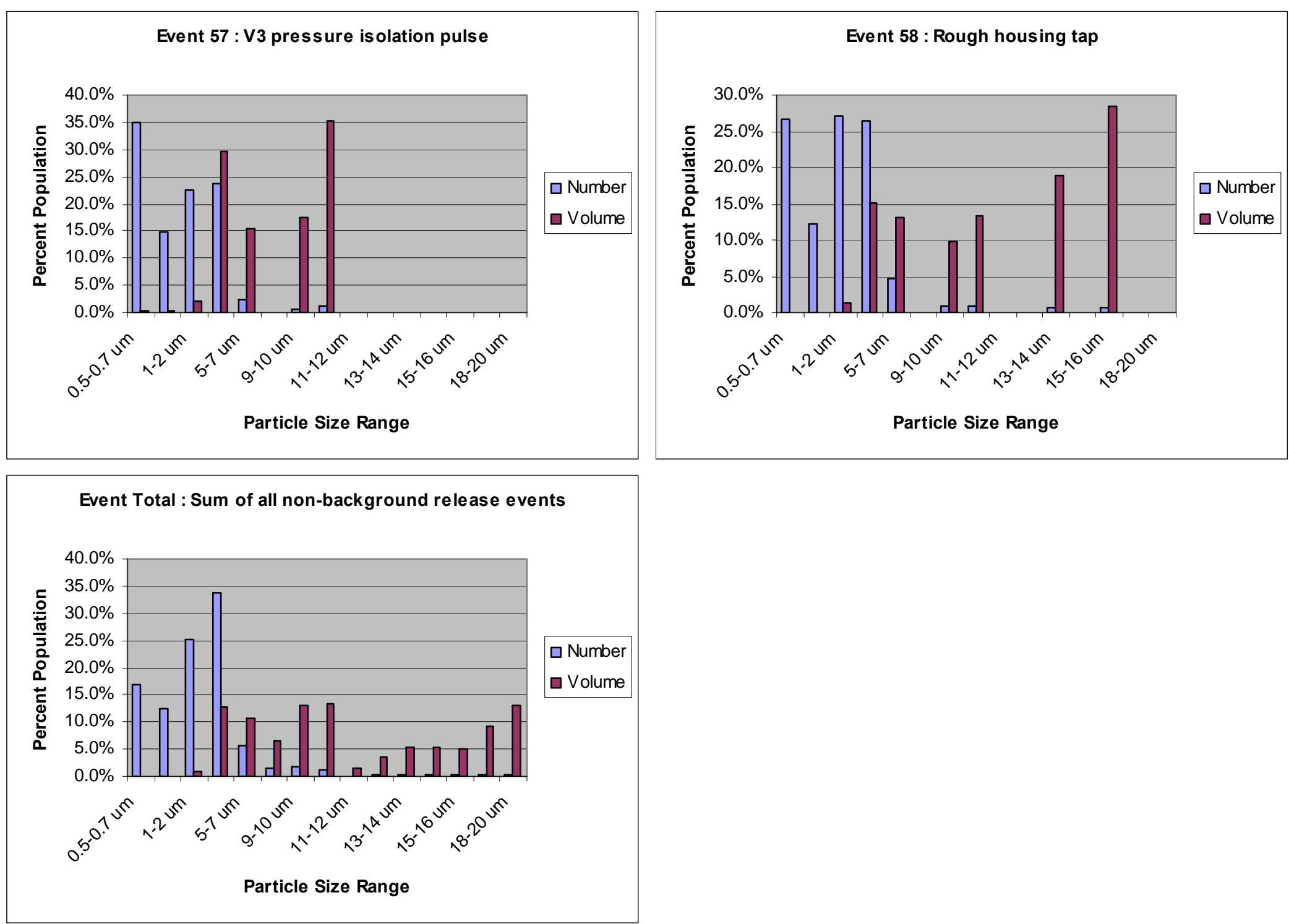

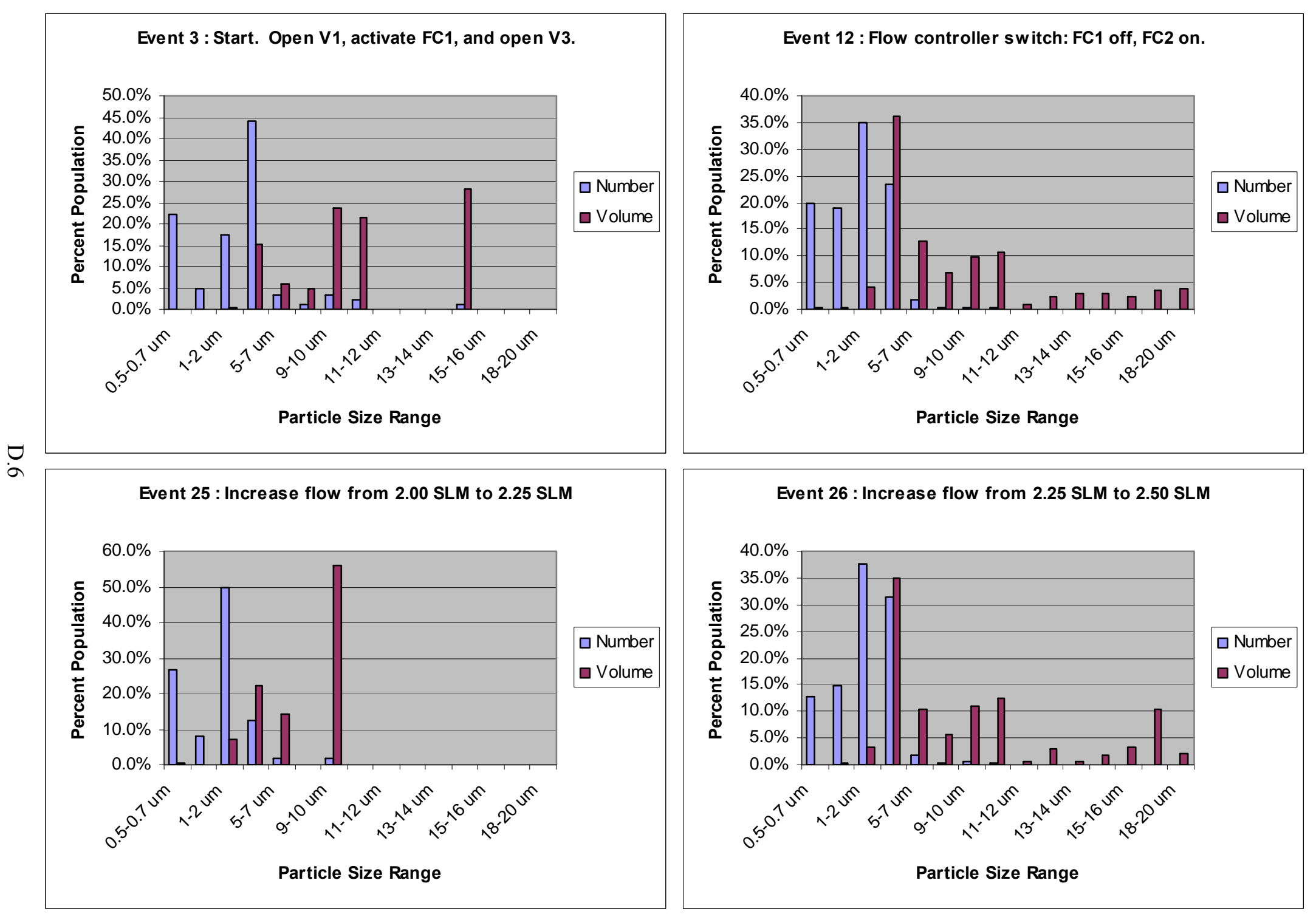

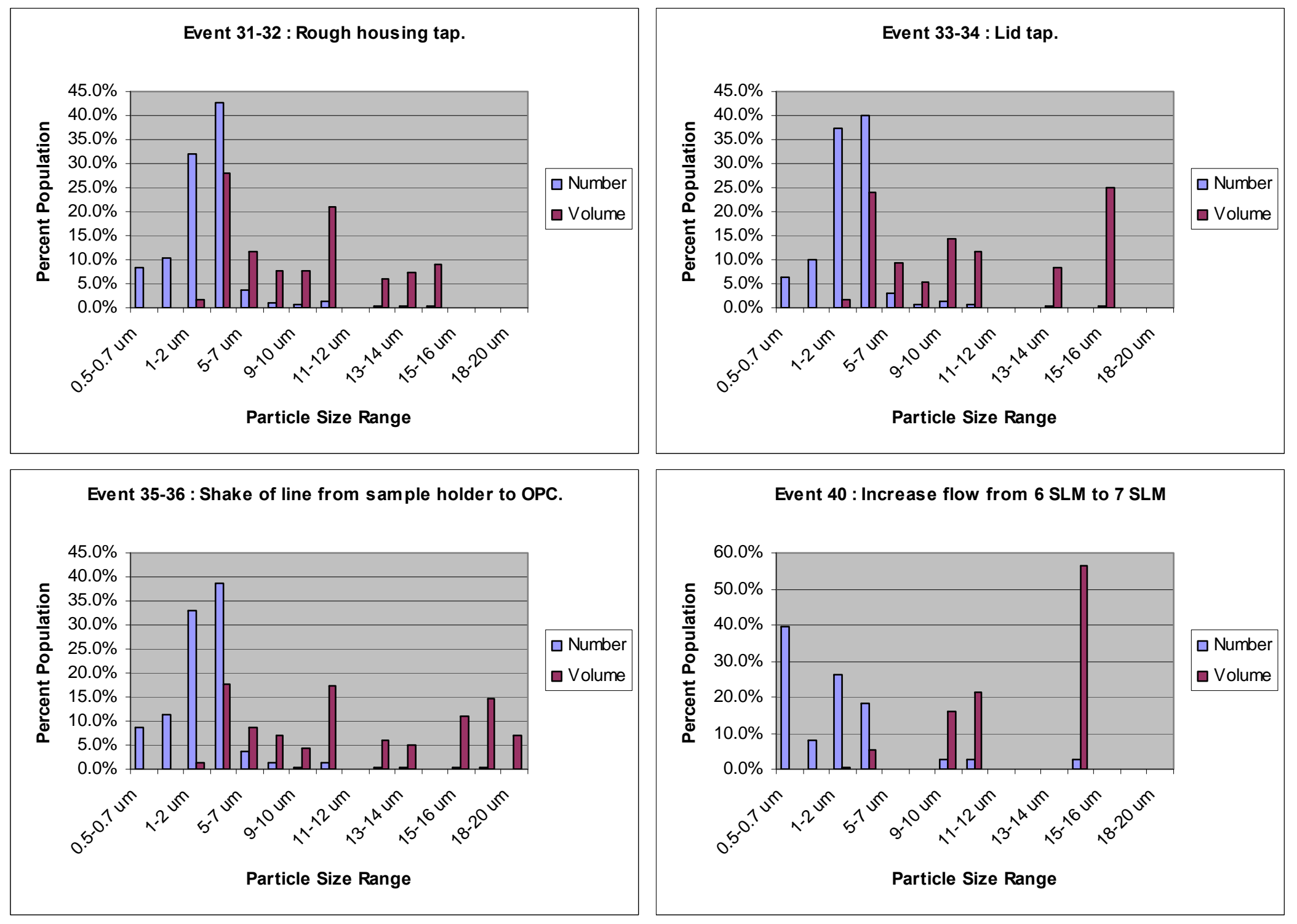

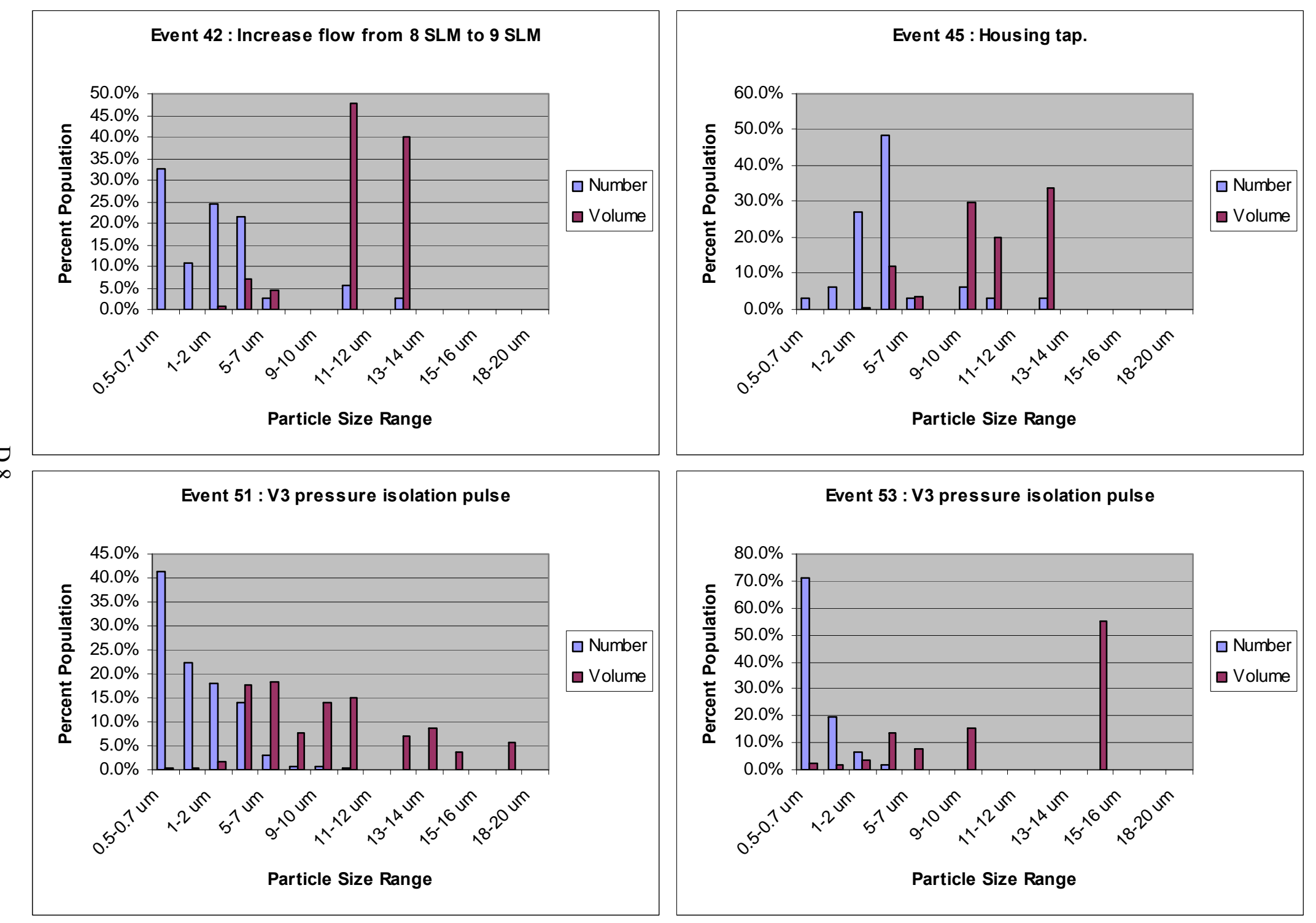


\section{5-LOW}
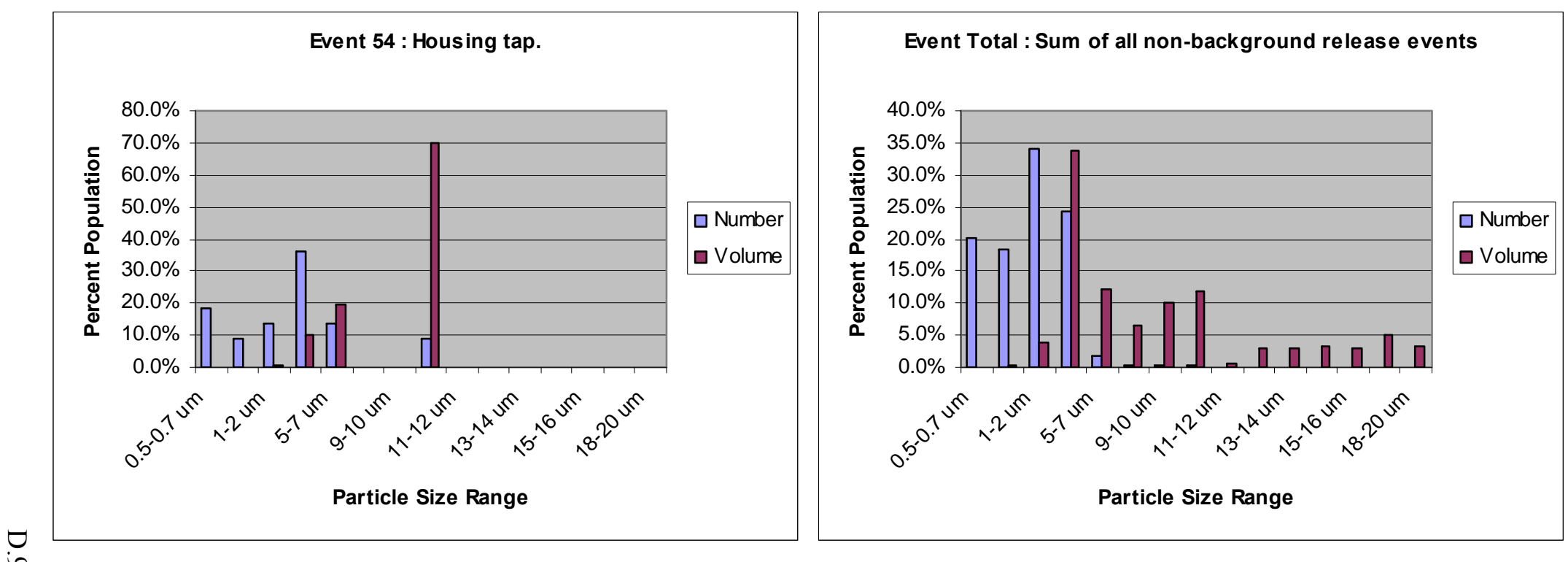

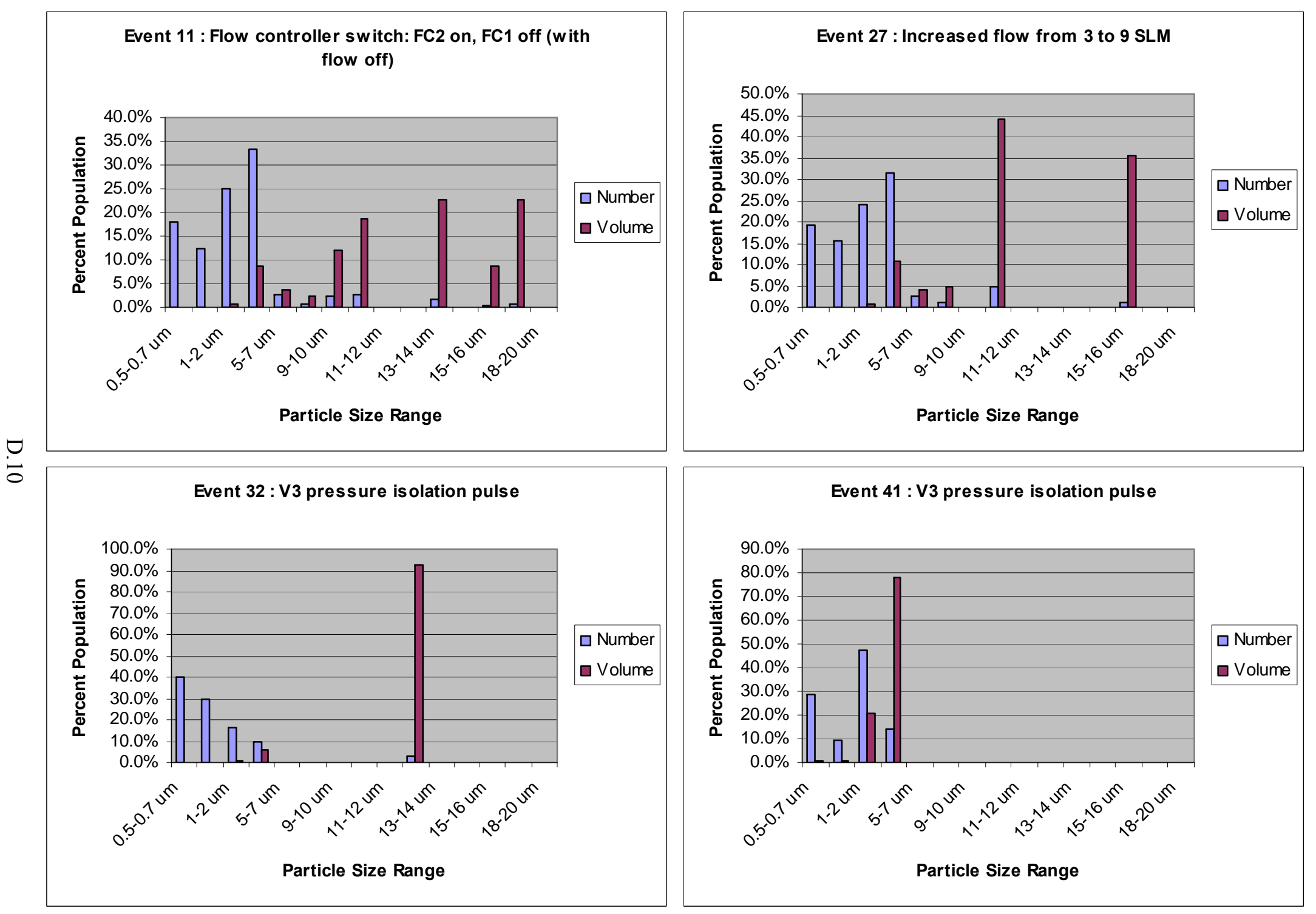

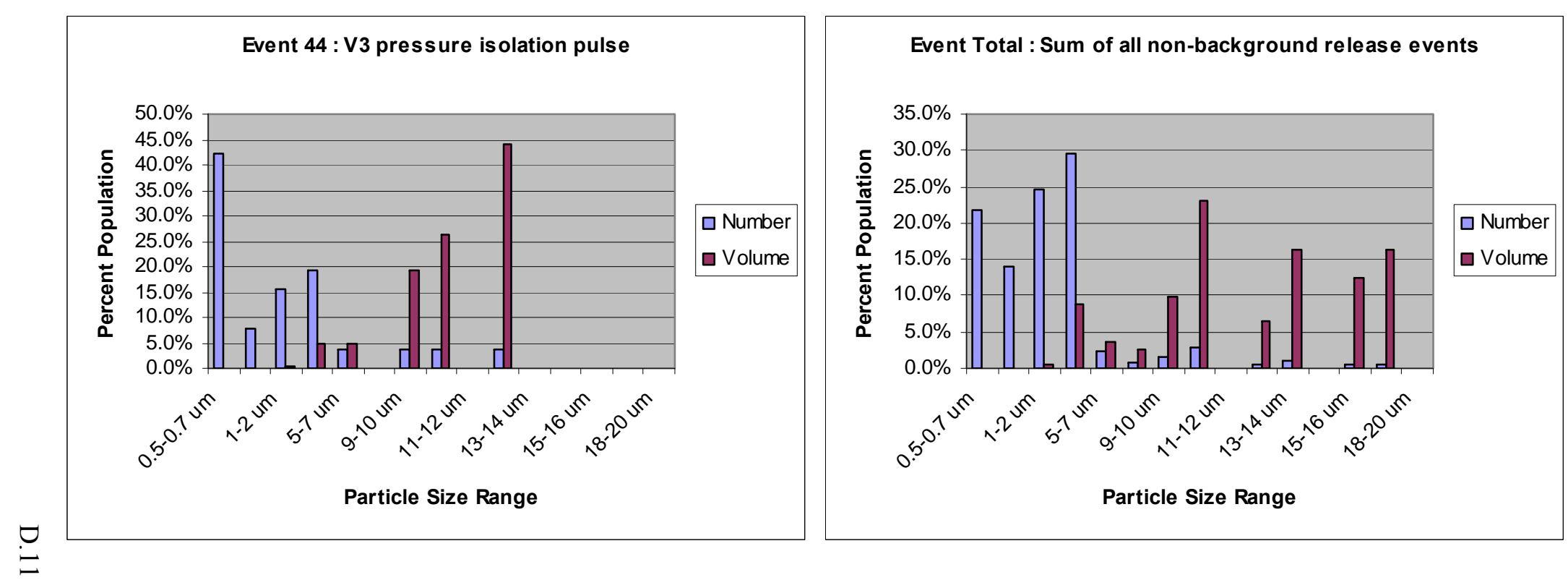

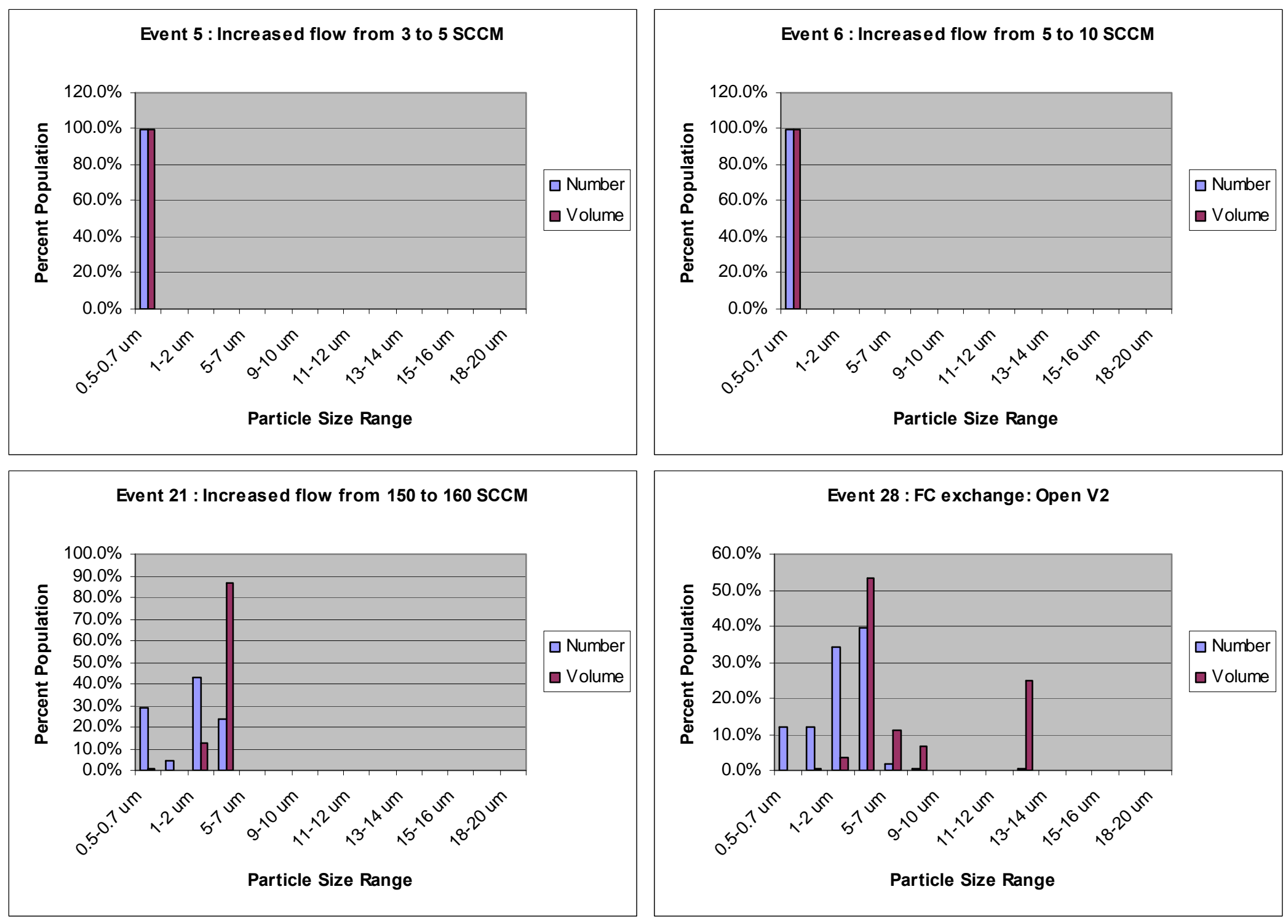

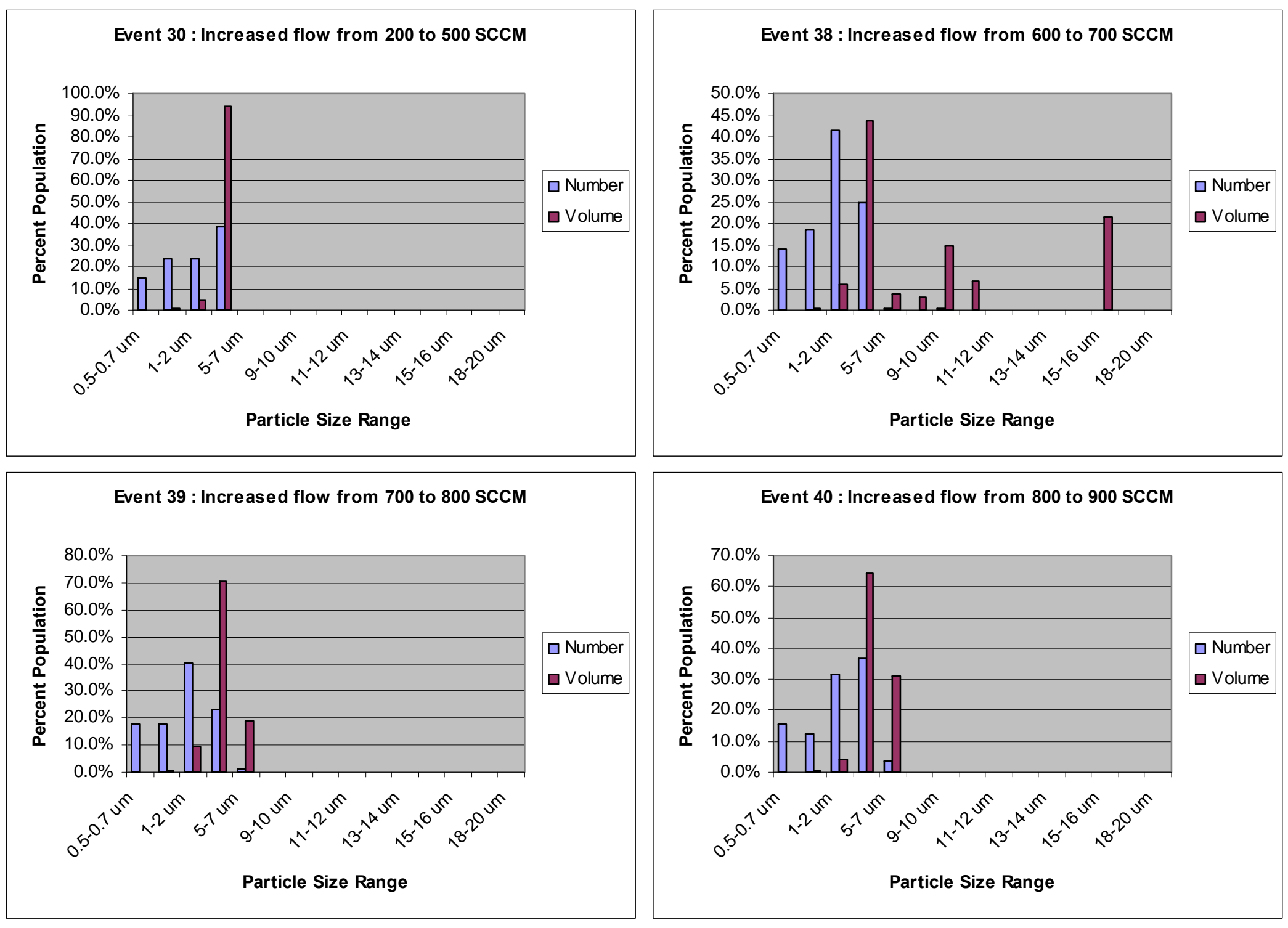

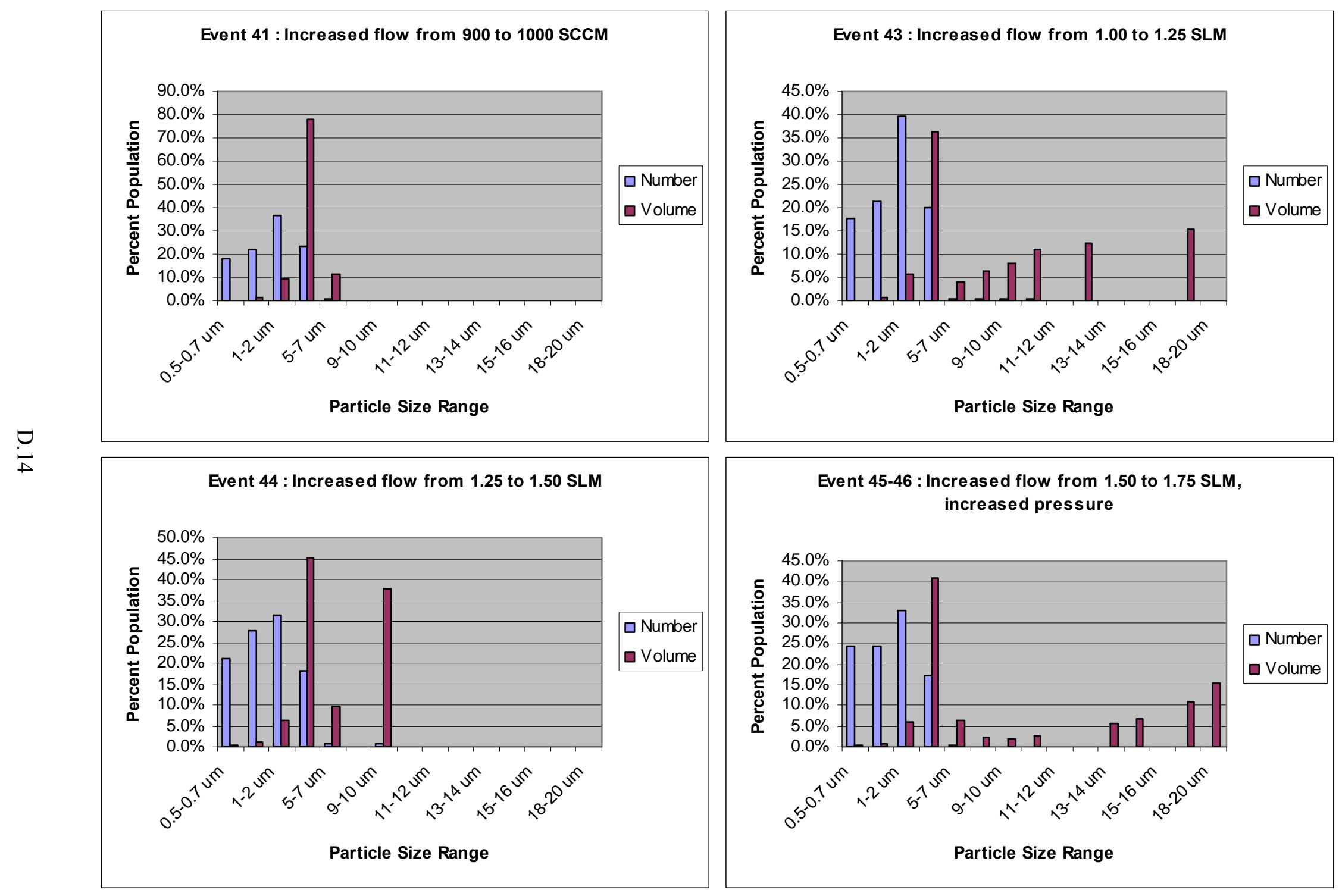

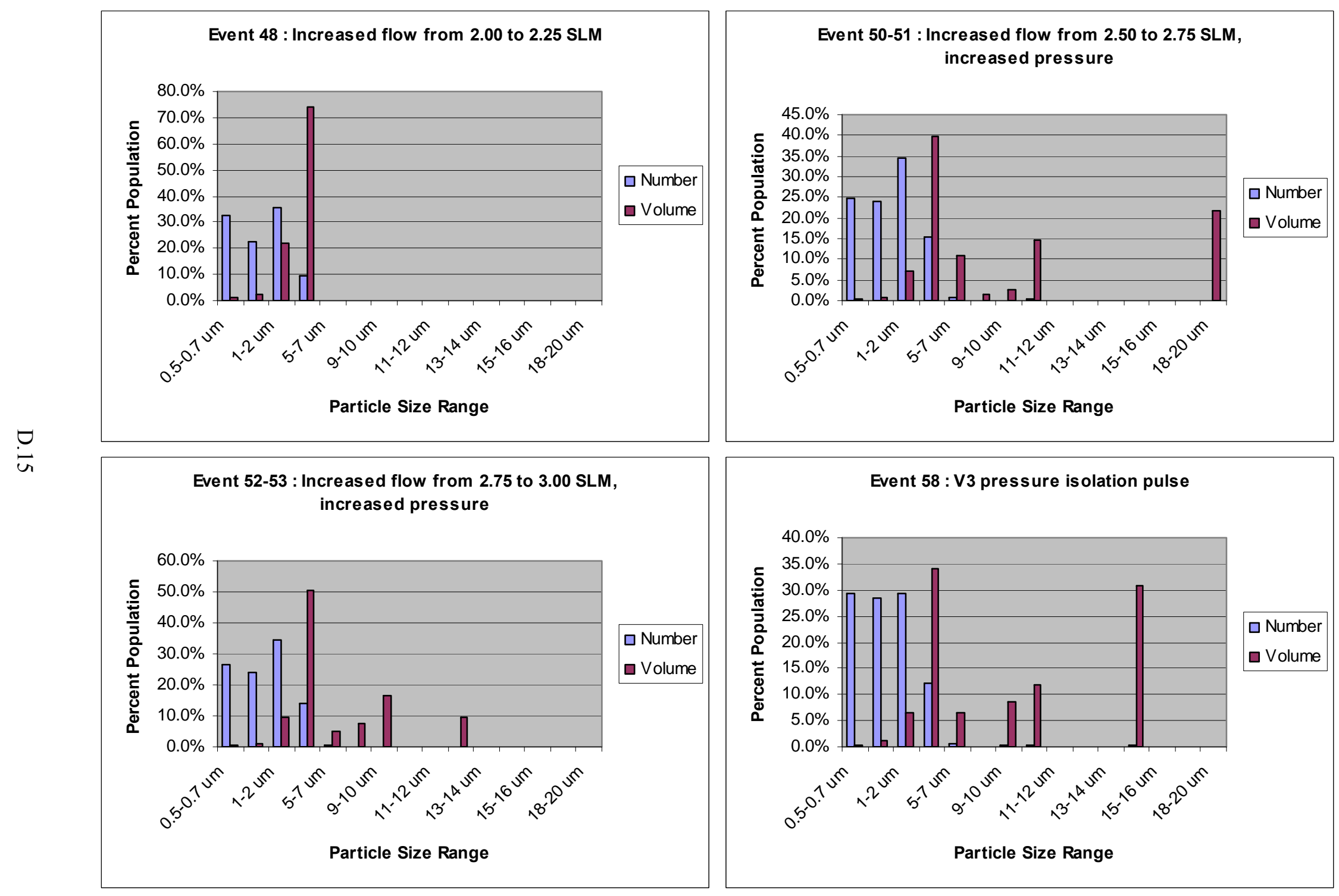

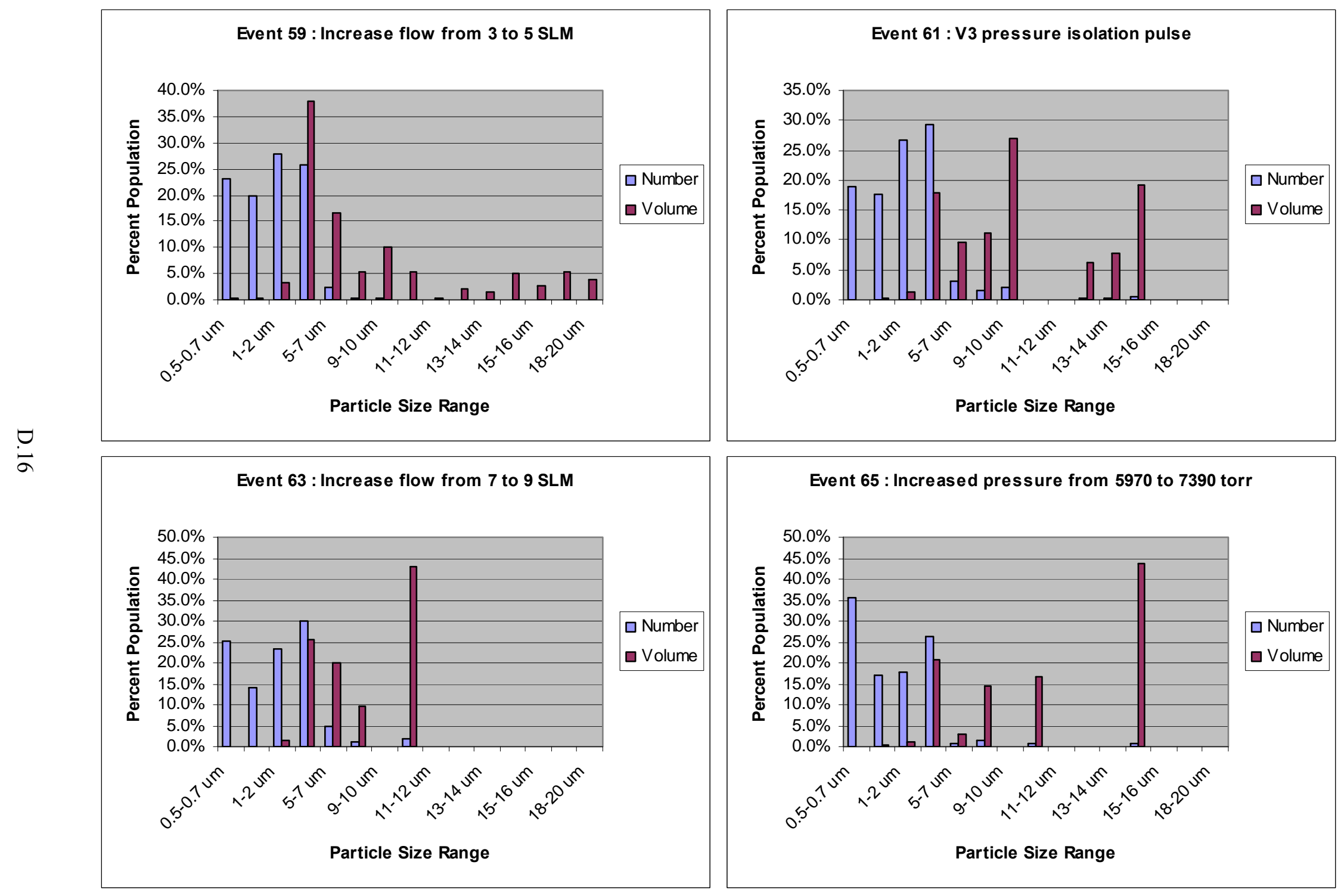


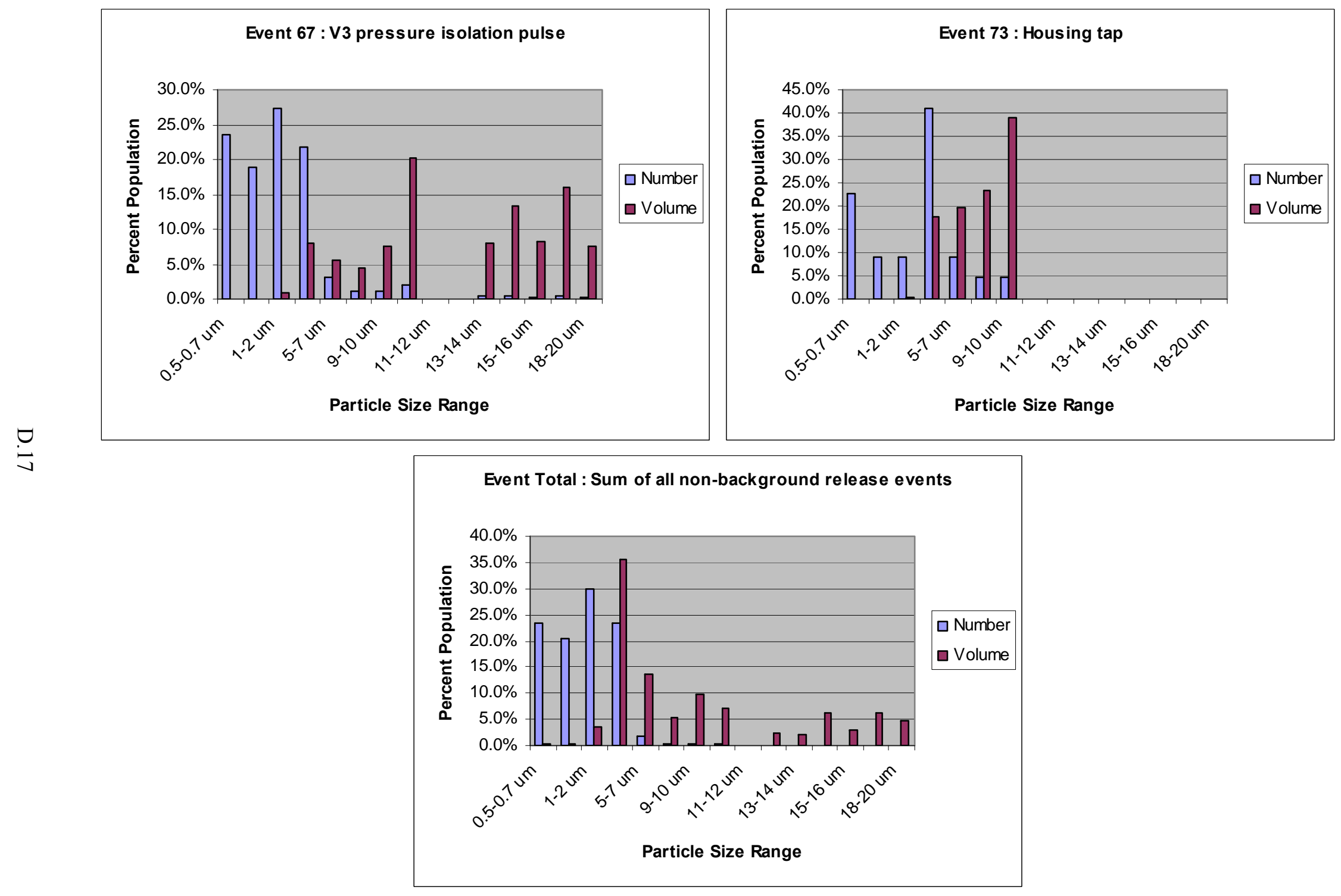


PNNL-17275, Rev. 1

\section{Distribution}

No. of

Copies

OFFSITE

\author{
8Bechtel SAIC Company, LLC \\ 1180 Town Center Drive \\ Las Vegas, NV 89144 \\ Attn: Mark Wisenburg (2) \\ Sen-Sung Tsai (6)
}

\begin{abstract}
No. of
Copies
\end{abstract}

\section{ONSITE}

10 Pacific Northwest National Laboratory

\begin{tabular}{ll}
\hline BD Hanson (5) & P7-27 \\
RC Daniel & P7-22 \\
RS Wittman & P7-27 \\
AM Casella & P7-27 \\
PJ MacFarlan & P7-27 \\
RW Shimskey & P7-27
\end{tabular}

Distr. 1 